\title{
Note on the bundle geometry of field space, variational connections, the dressing field method, \& presymplectic structures of gauge theories over bounded regions
}

\author{
J. François, N. Parrini and N. Boulanger \\ Service de Physique de l'Univers, Champs et Gravitation, Université de Mons - UMONS, \\ 20 Place du Parc, B-7000 Mons, Belgique \\ E-mail: Jordan.FRANCOIS@umons .ac.be, Noemie.PARRINI@umons.ac.be, \\ Nicolas. BOULANGER@umons.ac. be
}

ABSTRACT: In this note, we consider how the bundle geometry of field space interplays with the covariant phase space methods so as to allow to write results of some generality on the presymplectic structure of invariant gauge theories coupled to matter. We obtain in particular the generic form of Noether charges associated with field-independent and fielddependent gauge parameters, as well as their Poisson bracket. We also provide the general field-dependent gauge transformations of the presymplectic potential and 2-form, which clearly highlights the problem posed by boundaries in generic situations. We then conduct a comparative analysis of two strategies recently considered to evade the boundary problem and associate a modified symplectic structure to a gauge theory over a bounded region: namely the use of edge modes on the one hand, and of variational connections on the other. To do so, we first try to give the clearest geometric account of both, showing in particular that edge modes are a special case of a differential geometric tool of gauge symmetry reduction known as the "dressing field method". Applications to Yang-Mills theory and General Relativity reproduce or generalise several results of the recent literature.

KEYwords: Differential and Algebraic Geometry, Gauge Symmetry

ArXiv ePrint: 2109.07159 


\section{Contents}

1 Introduction 1

2 Geometry of field space $\quad 4$

2.1 Field space as a principal bundle 4

$\begin{array}{lll}2.2 & \text { Variational connections on field space } & 12\end{array}$

$\begin{array}{lll}2.2 .1 & \text { Variational Ehresmann connections } & 12\end{array}$

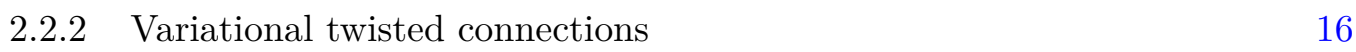

$\begin{array}{ll}2.3 & \text { The dressing field method } \\ & 17\end{array}$

2.3.1 Field-dependent dressing fields and variational connections 22

3 Presymplectic structures of matter coupled gauge theories over bounded regions

3.1 Covariant phase space formalism 24

3.2 Presymplectic structure of invariant matter coupled gauge theories 26

$\begin{array}{lll}3.3 & \text { Applications } & 33\end{array}$

$\begin{array}{lll}\text { 3.3.1 Coupled Yang-Mills theory } & 34\end{array}$

$\begin{array}{lll}3.3 .2 & \text { Coupled 4D gauge gravity } & 36\end{array}$

4 Basic presymplectic structures $\quad 41$

4.1 Via variational connections 42

4.2 Via dressing fields 44

$\begin{array}{lll}4.3 & \text { Applications } & 48\end{array}$

4.3.1 Yang-Mills theory $\quad 48$

$\begin{array}{lll}4.3 .2 & 4 \mathrm{D} \text { gauge gravity } & 53\end{array}$

$\begin{array}{lll}5 & \text { Conclusion } & 61\end{array}$

A Proof of formula $(2.27) \quad \mathbf{6 2}$

B The Abbott-Deser derivation of charges in YM theory 64

C Commutation relations with the extended bracket $(3.20) \quad 65$

\section{Introduction}

One motivation for the introduction of covariant phase space methods, at least in some of the foundational literature [1-3], is to associate a symplectic structure to a gauge field theory - given by a Lagrangian $L$ - over some region $\Sigma$ of spacetime $M$, and so doing while keeping spacetime symmetries manifest (with in mind a possible covariant canonical 
or geometric quantization). See [4] for a review with both historical context and discussions of related approaches. Usually the field space $\Phi$ is considered the configuration space of the theory, the space of solutions $\mathcal{S}$ is the phase space while its quotient by the gauge group $\mathcal{H}$ of the theory, $\mathcal{M}_{\mathcal{S}}:=\mathcal{S} / \mathcal{H}$ of the field equations, is the reduced phase space. The symplectic structure is complete once a symplectic 2 -form on $\mathcal{M}_{\mathcal{S}}$ is given. From the functional variation of $L$, one derives the presympletic potential $\boldsymbol{\theta}_{\Sigma}$ which is a 1 -form on field space, and its functional variation defines the associated presymplectic 2-form $\boldsymbol{\Theta}_{\Sigma}$ on $\Phi$. When, on-shell, the gauge directions are in its kernel, the latter descends to a symplectic 2 -form on $\mathcal{M}_{\mathcal{S}}$. This is the case when the region $\Sigma$ has no boundary or when adequate fall-off conditions for the fields $\phi$ are specified. But when $\partial \Sigma \neq \emptyset$ and there are no good reasons to assume $\phi=0_{\mid \partial \Sigma}$, neither $\boldsymbol{\theta}_{\Sigma}$ nor $\boldsymbol{\Theta}_{\Sigma}$ descend on the reduced phase space. We may refer to this as the boundary problem. It naturally arises e.g. when one considers the decomposition of a well-defined symplectic structure for a boundaryless region $\Sigma$ into symplectic sub-structures associated with an arbitrary partition of $\Sigma$ into subregions $\cup_{i} \Sigma_{i}$ sharing fictitious boundaries $\partial \Sigma_{i}$ - a classical analogue of the problem of factorising the Hilbert space of a quantum system into Hilbert subspaces, which in turn is closely related to the topic of entanglement entropy.

In the wake of renewed interest in this issue, over the past few years two strategies have been proposed to deal with this boundary problem in Yang-Mills theory and General Relativity: the "edge modes" strategy as introduced by Donnelly \& Freidel in [5], and the use of variational connections on field space as advocated by Gomez \& Riello first in [6] and further developed in [7-9] (see also [10-13]). Both essentially aim at providing a modified presymplectic structure that descends onto $\mathcal{M}_{\mathcal{S}}$ and that we will call basic for reasons to be made clear in due time.

The former strategy, explored in various contexts [14-17], considers an extended phase space comprising new degrees of freedom at $\partial \Sigma$ — the edge modes - entering boundary counterterms added to $\boldsymbol{\theta}_{\Sigma}$ and $\boldsymbol{\Theta}_{\Sigma}$ enforcing their vanishing along gauge directions. According to its proponents, the main virtue of edge modes beyond addressing the boundary issue is that they reveal new physical symmetries, sometimes called "surface symmetries", to which charges can be associated with and whose Poisson algebra encodes important kinematical information. ${ }^{1}$ For this reason, it has been suggested that edge modes provide a new angle for a quantum gravity program [23-25].

The use of connections received less systematic attention, but it has the advantage of possibly relying only on the resources of the original field space, without the need for introducing extra d.o.f. Its principal advocates also remarked that modified presymplectic structure obtained via a connection seems to generalise the one obtained via edge modes. No equivalent of the surface symmetries of edge mode seems to exist in this approach though. However, it was noted that its own special merit was to connect with the literature on dressings in gauge theory - à la Dirac, see e.g. [26, 27] — and in particular to reproduce the DePaoli-Speziale (DPS) "dressing 2-form" [28-30] relating the presymplectic potentials of the tetrad and metric formulations of GR.

\footnotetext{
${ }^{1}$ For other works on extended phase spaces by edge modes, or edge states, in another sense of the term i.e. true boundary d.o.f. - see e.g. [18-20], and in the context of asymptotic boundaries and gravitational waves memory effects see [21, 22]
} 
It would seem that a careful comparative analysis of both approaches weighting their respective strengths and their overlaps would be useful, clarifying the conceptual landscape and establishing clear bridges within the existing literature. Doing so is one of the objectives of this paper.

The difference in degree of formalisation would be a first hurdle to clear, the second approach being a priori much more geometric than the first. Fortunately, philosophers of physics interested in foundational issues in gauge field theories first remarked [31] see also [32] — that the edge mode strategy could be seen as a special application of a geometric tool designed to reduce gauge symmetries called the "dressing field method" (DFM). In [33], the case was made thoroughly that it is indeed so, and that many conceptual clarifications ensue.

A second hurdle is that both approaches have been mainly detailed via applications to specific examples. Even though one could compare the outputs of both methods in common examples, e.g. in the YM case as was done in [9] or [13], we aim for a more ambitious goal: we want to confront these two approaches in their most general versions. To do so, we must first conduct the most general analysis possible of the relevant presymplectic structure of gauge theories coupled to matter, restricting ourselves - for reasons to be clarified later - to theories that are strictly gauge invariant. This is our second goal in this note. To be specific, we will provide the generic form of the Noether charges associated with both field-independent and field-dependent gauge parameters, as well as their off-shell Poisson bracket. We also derive the general field-dependent gauge transformations of the presymplectic potential and 2-form, from which the boundary problem will appear in its most general form. Doing all this, we will have generalised some of the results of [33] valid only for pure gauge theories. ${ }^{2}$

To achieve this, we must start one step higher in generality. Indeed, we find indispensable for conceptual clarity and technical efficiency to clearly articulate how the bundle geometry of field space interplays with covariant phase space methods. We are thus lead to spell out the $\mathcal{H}$-principal bundle structure of field space $\Phi$, whose base is the moduli space $\mathcal{M}:=\Phi / \mathcal{H}$ where gauge-invariant quantities live. Variational forms on $\Phi$ projecting to well-defined objects on the base are the so-called basic forms. Solving the boundary problem thus means identifying strategies to construct basic versions of the initial presymplectic structure of a gauge theory. The broad issue is then to build the basic counterpart of a given variational form on $\Phi$. Variational principal connections and the DFM are two methods to achieve this goal, as we propose to show.

So, let us recap what we intend to do in this note: in section 2, we describe in some details the bundle geometry of field space to give the most conceptual clarity on the kinematics at play. We remind the definition of variational Ehresmann connections, which are fit for the analysis of invariant theories - our main focus. As an hopefully informative aside, we also define a generalisation known as twisted connections that are well adapted to non-invariant theories. Then we review the DFM, introducing the notion of field-dependent

\footnotetext{
${ }^{2}$ Where was also conducted the analysis of pure gauge theories that are non-invariant, but whose classical gauge anomaly is $d$-exact. See the conclusion for further comments.
} 
dressing fields. We show how basic forms are built via connections and dressings, and we stress the relations between the two approaches.

In section 3 we show how the bundle geometry of $\Phi$ interlaces with covariant phase space methods, thereby allowing to write down the above mentioned general results on the presymplectic structure of invariant, matter coupled, gauge theories. We remark that, once such general formulae are given, the only computation needed for applications to specific examples is to derive the field equations and presymplectic potential from the Lagrangian at hand. We illustrate the procedure in YM theory and in a Cartan geometric formulation of GR, and recover standard results. We also say a word about the generic physical interpretation of a Noether charge, relying on the affine structure of the space of connections to split it as a background contribution plus a measurable contribution, thereby connecting with the definition of charges of Abbott \& Deser [34, 35]. We therefore emphasize a geometric aspect of (generalised) Noether charges, as is typically done with covariant phase space methods, which is complementary to the intrinsically cohomological nature of charges [36].

Finally, in section 4 we give the most general versions of the basic presymplectic structure obtained respectively via connections and via the DFM. We highlight the geometric origin of their structural similarities and stress the crucial differences, in particular regarding how ambiguities arise in both schemes. This last point is relevant to better understand the meaning of surface symmetries in the edge mode literature, as we show that these and coordinate transformations in GR are on the same conceptual footing from the DFM viewpoint. Applications to YM theory and GR allow to recover many results of the literature cited above. In particular we show how the DFM gives from first principles the unambiguous link between the presymplectic structures of GR in the tetrad formulation and in the metric formulation, thereby generalising the DPS dressing- 2 form.

In our conclusion 5, beside giving a quick review of our results, we hint at generalisations that we intend to pursue. Technical details are completed in appendices. For comparison with the body of the text, we also give a reformulation in terms of differential forms of the Abbott-Deser algorithm for defining charges in YM theory.

\section{Geometry of field space}

In this section we give a sense of the bundle geometry of field space. As it involves infinite dimensional vector spaces and manifolds, we defer to the relevant literature [37, 38] to back the soundness of extending any standard notion defined in the finite dimensional context to its infinite dimensional counterpart. We aim for a correct conceptual picture rather than a perfectly mathematically rigorous one.

\subsection{Field space as a principal bundle}

The configuration space of a gauge theory is the field space $\Phi=\mathcal{A} \times \Gamma(E)$, where $\mathcal{A}$ is the space of (Ehresmann or Cartan) connections on a $H$-bundle $\mathcal{P}$ over spacetime $M$ ( $H$ a Lie group), i.e. gauge potentials, and $\Gamma(E) \simeq \Omega_{\mathrm{eq}}^{0}(\mathcal{P}, V)$ is the space of sections of bundles $E$ associated to $\mathcal{P}$ via representations $(V, \rho)$ of $H$, i.e. matter fields. 
The space $\Phi$ is an infinite dimensional Banach manifold, so is the gauge group $\mathcal{H}$ as an infinite dimensional Lie group. Under proper restrictions (on either $\Phi$ or $\mathcal{H}$ [39-44]), the moduli space $\mathcal{M}=\Phi / \mathcal{H}$ is well-behaved as a manifold. Then, $\Phi$ is a principal bundle over $\mathcal{M}$ with structure group $\mathcal{H}$, whose right action we denote $(\phi, \gamma) \mapsto R_{\gamma} \phi:=\phi^{\gamma}{ }^{3}$ Explicitly of course, $\phi^{\gamma}=\left(A^{\gamma}, \varphi^{\gamma}\right)=\left(\gamma^{-1} A \gamma+\gamma^{-1} d \gamma, \rho(\gamma)^{-1} \varphi\right)$, as $\mathcal{A}$ and $\Gamma(E)$ are separately $\mathcal{H}$ principal bundles. The gauge orbit $\mathcal{O}_{\mathcal{H}}[\phi]$ of $\phi \in \Phi$ is a fiber over the gauge class $[\phi] \in \mathcal{M}$. The projection $\pi: \Phi \rightarrow \mathcal{M}, \phi \mapsto \pi(\phi)=[\phi]$, is s.t. $\pi \circ R_{\gamma}=\pi$.

As a bundle, $\Phi$ is locally trivial: given $\mathcal{U} \subset \mathcal{M}, \Phi_{\mid \mathcal{U}} \simeq \mathcal{U} \times \mathcal{H}$. A trivialising (or local) section $\boldsymbol{\sigma}: \mathcal{U} \rightarrow \Phi,[\phi] \mapsto \boldsymbol{\sigma}([\phi])$, is such that that $\pi \circ \boldsymbol{\sigma}=\mathrm{id} \mathcal{U}$. If $\exists$ a global section $\boldsymbol{\sigma}: \mathcal{M} \rightarrow \Phi$, then the bundle is trivial, $\Phi \simeq \mathcal{M} \times \mathcal{H}$. Given $\boldsymbol{\sigma}_{i}$ and $\boldsymbol{\sigma}_{j}$ sections over $\mathcal{U}_{i}, \mathcal{U}_{j} \subset \mathcal{M}$ s.t. $\mathcal{U}_{i} \cap \mathcal{U}_{j} \neq \emptyset$, on the overlap $\boldsymbol{\sigma}_{j}=R_{\boldsymbol{h}_{i j}} \boldsymbol{\sigma}_{i}=\boldsymbol{\sigma}_{i}^{\boldsymbol{h}_{i j}}$ where $\boldsymbol{h}_{i j}: \mathcal{U}_{i} \cap \mathcal{U}_{j} \rightarrow \mathcal{H}$ is a transition function. The set $\left\{\boldsymbol{h}_{i j}\right\}$ of transition functions subordinated to a covering $\left\{\mathcal{U}_{i}\right\}_{i \in I \subset \mathbb{N}}$ of $\mathcal{M}$ are local data from which it is possible to reconstruct the bundle $\Phi$. A trivialising section $\boldsymbol{\sigma}$ selects a single representative by gauge orbit $\boldsymbol{\sigma}([\phi])=\phi \in \mathcal{O}_{\mathcal{H}}[\phi]$, $\forall[\phi] \in \mathcal{U}$, it is thus a gauge choice (a gauge fixing). ${ }^{4}$ A transition function $\boldsymbol{h}([\phi]) \in \mathcal{H}$, that allows to switch from one gauge choice to another, is a gauge transformation that depends only on the gauge class $[\phi]$ of the gauge fields $\phi$. This is close to the notion of field-dependent gauge transformations often encountered in the literature, but not quite the same thing yet. Another natural geometric concept, described next, better captures this notion.

The natural transformation group of $\Phi$ is its automorphism group $\operatorname{Aut}(\Phi):=$ $\left\{\boldsymbol{\Psi}: \Phi \rightarrow \Phi \mid \boldsymbol{\Psi} \circ R_{\gamma}=R_{\gamma} \circ \boldsymbol{\Psi}\right\}$. Only $\boldsymbol{\Psi} \in \operatorname{Aut}(\Phi)$ project to well-defined $\boldsymbol{\psi} \in \operatorname{Diff}(\mathcal{M})$, which is the physical transformation group acting on physical states. As usual, the subgroup of vertical automorphisms $\operatorname{Aut}_{v}(\Phi):=\{\boldsymbol{\Psi} \in \operatorname{Aut}(\Phi) \mid \pi \circ \boldsymbol{\Psi}=\pi\}$ is isomorphic to the gauge group $\mathcal{H}:=\left\{\gamma: \Phi \rightarrow \mathcal{H} \mid R_{\gamma}^{\star} \gamma(\phi)=\gamma^{-1} \gamma(\phi) \gamma\right\}$ by the correspondance $\boldsymbol{\Psi}(\phi)=R_{\gamma(\phi)} \phi=\phi^{\gamma(\phi)}$. Now, the gauge group $\mathcal{H}$ is indeed the geometric underpinning of the notion of field-dependent gauge transformations. We have the characteristic short exact sequence (SES) of groups associated with the bundle $\Phi$,

$$
0 \longrightarrow \operatorname{Aut}_{v}(\Phi) \simeq \mathcal{H} \longrightarrow \stackrel{\iota}{\longrightarrow} \operatorname{Aut}(\Phi) \stackrel{\tilde{\pi}}{\longrightarrow} \operatorname{Diff}(\mathcal{M}) \longrightarrow 0
$$

Without a splitting of this SES, one cannot decompose uniquely an element of Aut $(\Phi)$ into a vertical automorphism and a diffeomorphism of the physical configuration space $\mathcal{M}$.

Tangent and vertical bundles. The connection space $\mathcal{A}$ is an affine space modelled on $\Omega_{\text {tens }}^{1}(\mathcal{P}, \operatorname{Lie} H)$, while $\Gamma(E) \simeq \Omega_{\text {tens }}^{0}(\mathcal{P}, V)$ is a vector space. Therefore, the tangent space at $\phi \in \Phi$ is $T_{\phi} \Phi=T_{A} \mathcal{A} \oplus T_{\varphi} \Gamma(E) \simeq \Omega_{\text {tens }}^{1}(\mathcal{P}, \operatorname{Lie} H) \oplus \Omega_{\text {tens }}^{0}(\mathcal{P}, V)$. A generic vector $\boldsymbol{X}_{\phi} \in T_{\phi} \Phi$ with flow $f_{\tau}: \Phi \rightarrow \Phi, \phi \mapsto f_{\tau}(\phi)=\left(f_{\tau}^{A}(\phi), f_{\tau}^{\varphi}(\phi)\right)$ and $f_{\tau=0}(\phi)=\phi$, is s.t. $\quad \boldsymbol{X}_{\phi}=\left.\frac{d}{d \tau} f_{\tau}(\phi)\right|_{\tau=0}$. Formally, we can write a vector field $\boldsymbol{X} \in \Gamma(T \Phi)$ as a variational differential operator on $C^{\infty}(\Phi): \quad \boldsymbol{X}_{\phi}=\boldsymbol{X}(\phi) \frac{\delta}{\delta \phi}$, with $\boldsymbol{X}(\phi)=\left.\frac{d}{d \tau} f_{\tau}(\phi)\right|_{\tau=0}=$

\footnotetext{
${ }^{3}$ Since $\left(\phi^{\gamma}\right)^{\gamma^{\prime}}=\left(\phi^{\gamma^{\prime}}\right)^{\gamma^{\gamma^{\prime}}}=\left(\phi^{\gamma^{\prime}}\right)^{\gamma^{\prime-1} \gamma \gamma^{\prime}}=\phi^{\gamma \gamma^{\prime}}$, this is indeed a right action: $R_{\gamma^{\prime}} \circ R_{\gamma}=R_{\gamma \gamma^{\prime}}$.

${ }^{4}$ If the factor $\mathcal{A}$ in $\Phi$ is the space of connections of a $\mathrm{SU}(N)$-bundle $P$ over a compact spacetime manifold $M$, then no such global section exists (this is the Gribov ambiguity [45]). As Singer says in the abstract of his paper [39], in this case "no gauge fixing is possible".
} 
$\left(\left.\frac{d}{d \tau} f_{\tau}^{A}(\phi)\right|_{\tau=0},\left.\frac{d}{d \tau} f_{\tau}^{\varphi}(\phi)\right|_{\tau=0}\right)=\boldsymbol{X}^{A}(\phi)+\boldsymbol{X}^{\varphi}(\phi) \in \Omega_{\text {tens }}^{1}(\mathcal{P}, \operatorname{Lie} H) \oplus \Omega_{\text {tens }}^{0}(\mathcal{P}, V)$ the 'components' of $\boldsymbol{X} .{ }^{5}$ Only right-invariant vector fields, $\Gamma_{\mathcal{H}}(T \Phi):=\left\{\boldsymbol{X} \in \Gamma(T \Phi) \mid R_{{ }_{\star}} \boldsymbol{X}_{\phi}=\boldsymbol{X}_{\phi^{\gamma}}\right\}$, project to well-defined vector fields on the base, and $\pi_{\star}: \Gamma_{\mathcal{H}}(T \Phi) \rightarrow \Gamma(T \mathcal{M})$ is a morphism of Lie algebras. The flow of a right-invariant vector field belongs to $\operatorname{Aut}(\Phi)$, so that $\Gamma_{\mathcal{H}}(T \Phi) \simeq \operatorname{LieAut}(\Phi)$.

An element $\chi \in$ Lie $\mathcal{H}$ induces a vector

$$
\chi_{\phi}^{v}:=\left.\frac{d}{d \tau} \phi^{\exp (\tau \chi)}\right|_{\tau=0}=\left(\left.\frac{d}{d \tau} A^{\exp (\tau \chi)}\right|_{\tau=0},\left.\frac{d}{d \tau} \varphi^{\exp (\tau \chi)}\right|_{\tau=0}\right)=\left(D^{A} \chi,-\rho_{*}(\chi) \varphi\right)
$$

tangent to the fiber $\mathcal{O}_{\mathcal{H}}[\phi]$ at $\phi=(A, \varphi)$. All such vectors span the vertical subbundle $V \Phi \subset T \Phi$, and a vertical vector field $\chi^{v} \in \Gamma(V \Phi)$ is s.t. $\pi_{\star} \chi^{v}=0$ and $R_{\gamma \star} \chi_{\phi}^{v}=\left(\gamma^{-1} \chi \gamma\right)_{\phi^{\gamma}}^{v}$. We have the injective morphism of Lie algebras Lie $\mathcal{H} \rightarrow \Gamma(V \Phi)$. Similarly, elements of the Lie algebra of the gauge group Lie $\mathcal{H}:=\left\{\chi: \Phi \rightarrow\right.$ Lie $\left.\mathcal{H} \mid R_{\gamma}^{\star} \chi=\gamma^{-1} \chi \gamma\right\}$ induce $\mathcal{H}$ right invariant vertical vector fields $\chi_{\phi}^{v}:=\left.\frac{d}{d \tau} \phi^{\exp (\tau \chi(\phi))}\right|_{\tau=0}=\left(D^{A} \chi(\phi),-\rho_{*}(\chi(\phi)) \varphi\right)$, s.t. $R_{\gamma \star} \boldsymbol{\chi}_{\phi}^{v}=\chi_{\phi^{\gamma}}^{v}$, so that the map Lie $\mathcal{H} \rightarrow \Gamma_{\mathcal{H}}(V \Phi)$ is a Lie algebra anti-isomorphism. Corresponding to (2.1) we have,

$$
0 \longrightarrow \Gamma_{\mathcal{H}}(V \Phi) \simeq \operatorname{Lie} \mathcal{H} \stackrel{\iota}{\longrightarrow} \Gamma_{\mathcal{H}}(T \Phi) \stackrel{\pi_{\star}}{\longrightarrow} \Gamma(T \mathcal{M}) \longrightarrow 0 .
$$

A splitting of this SES would allow to define (non-canonically) a notion of horizontality on $\Phi$ complementary to the verticality canonically given by the action of $\mathcal{H}$. This is what a choice of variational Ehresmann connection $\boldsymbol{\omega} \in \mathcal{A}_{\Phi}$ on $\Phi$ achieves. But depending on the problem at hand, one may instead need to endow $\Phi$ with a twisted variational connection $\tilde{\boldsymbol{\omega}} \in \tilde{\mathcal{A}}_{\Phi}$. See section 2.2 below.

A local section doesn't provide such a (global) splitting, yet it is useful to record its action: we have $\boldsymbol{\sigma}_{\star}: T_{[\phi]} \mathcal{M} \rightarrow T_{\boldsymbol{\sigma}([\phi])} \Phi, \boldsymbol{X}_{[\phi]} \mapsto \boldsymbol{\sigma}_{\star} \boldsymbol{X}_{[\phi]}$, and the pushforwards of $\boldsymbol{X}_{[\phi]} \in T_{[\phi]} \mathcal{M}$ by two sections $\boldsymbol{\sigma}$ and $\boldsymbol{\sigma}^{\prime}$ s.t. $\boldsymbol{\sigma}^{\prime}=R_{\boldsymbol{h}} \boldsymbol{\sigma}$, are related by

$$
\begin{aligned}
\boldsymbol{\sigma}_{\star}^{\prime} \boldsymbol{X}_{[\phi]} & =R_{\boldsymbol{h}([\phi]) \star}\left(\boldsymbol{\sigma}_{\star} \boldsymbol{X}_{[\phi]}\right)+\left\{\boldsymbol{h}^{-1} \boldsymbol{d} \boldsymbol{h}_{\mid[\phi]}\left(\boldsymbol{X}_{\phi}\right)\right\}_{\boldsymbol{\sigma}^{\prime}([\phi])}^{v} \\
& =R_{\boldsymbol{h}([\phi]) \star}\left(\boldsymbol{\sigma}_{\star} \boldsymbol{X}_{[\phi]}+\left\{\boldsymbol{d} \boldsymbol{h} \boldsymbol{h}_{[[\phi]}^{-1}\left(\boldsymbol{X}_{[\phi]}\right)\right\}_{\boldsymbol{\sigma}([\phi])}^{v}\right),
\end{aligned}
$$

where in the second equality we used the equivariance property of fundamental vertical vector fields. This result allows to define the gluing relations between local representatives on $\mathcal{M}$ of variational forms on $\Phi$, as we are about to see. To define the field-dependent gauge transformations of such forms, below, we need to record the action of $\operatorname{Aut}_{v}(\Phi) \simeq \mathcal{H}$ on $\Gamma(T \Phi)$ : the action of $\boldsymbol{\Psi} \in \mathbf{A u t}_{v}(\Phi)$ on a generic $\boldsymbol{X} \in \Gamma(T \Phi)$ is

$$
\boldsymbol{\Psi}_{\star} \boldsymbol{X}_{\phi}=R_{\gamma(\phi) \star} \boldsymbol{X}_{\phi}+\left\{\boldsymbol{\gamma}^{-1} \boldsymbol{d} \gamma_{\mid \phi}\left(\boldsymbol{X}_{\phi}\right)\right\}_{R_{\gamma(\phi)} \phi}^{v}=R_{\gamma(\phi) \star}\left(\boldsymbol{X}_{\phi}+\left\{\boldsymbol{d} \boldsymbol{\gamma} \boldsymbol{\gamma}_{\mid \phi}^{-1}\left(\boldsymbol{X}_{\phi}\right)\right\}_{\phi}^{v}\right) .
$$

Equations (2.3) and (2.4) are the exact analogues of their finite dimensional counterparts, and are proved in much the same way (the proof valid on $\mathcal{A}$ given in appendix B of [33] is easily adapted to $\Phi)$.

\footnotetext{
${ }^{5}$ So that $\boldsymbol{X}_{\phi}=\boldsymbol{X}(\phi) \frac{\delta}{\delta \phi}=\boldsymbol{X}^{A}(\phi) \frac{\delta}{\delta A}+\boldsymbol{X}^{\varphi}(\phi) \frac{\delta}{\delta \varphi}$. Integration over domains is tacit, and we also avoid generalised indices à la DeWitt [46].
} 
Variational differential forms. The de Rham complex on $\Phi$ is $\left(\Omega^{\bullet}(\Phi), \boldsymbol{d}\right)$ with $\boldsymbol{d}$ the variational exterior derivative of degree 1 , defined via a Koszul formula. The interior product $\iota: \Gamma(T \Phi) \times \Omega^{\bullet}(\Phi) \rightarrow \Omega^{\bullet-1}(\Phi),(\boldsymbol{X}, \boldsymbol{\alpha}) \rightarrow \iota_{\boldsymbol{X}} \boldsymbol{\alpha}=\boldsymbol{\alpha}(\boldsymbol{X}, \ldots)$, is a degree -1 derivation $\forall \boldsymbol{X}$. The Lie derivative $\boldsymbol{L}_{\boldsymbol{X}}:=\left[\iota_{\boldsymbol{X}}, \boldsymbol{d}\right]$, is thus a degree 0 derivation. ${ }^{6}$ It satisfies $\left[\boldsymbol{L}_{\boldsymbol{X}}, \iota_{\boldsymbol{Y}}\right]=\iota_{[\boldsymbol{X}, \boldsymbol{Y}]}$, and it is a Lie algebra morphism $\left[\boldsymbol{L}_{\boldsymbol{X}}, \boldsymbol{L}_{\boldsymbol{Y}}\right]=\boldsymbol{L}_{[\boldsymbol{X}, \boldsymbol{Y}]}$.

An exterior product $\wedge$ is defined as usual on the space $\Omega^{\bullet}(\Phi, A)$ of variational differential forms with values in an algebra $(\mathrm{A}, \cdot)$ - and kept tacit throughout for convenience so that $\left(\Omega^{\bullet}(\Phi, \mathrm{A}), \wedge, \mathrm{d}\right)$ is a differential graded algebra. The exterior product is not defined on $\Omega^{\bullet}(\Phi, \boldsymbol{V})$ where $\boldsymbol{V}$ is merely a vector space. But if $(\boldsymbol{V}, \rho)$ is a representation for $\mathcal{H}$, one defines the vector space of equivariant forms as $\Omega_{\mathrm{eq}}^{\bullet}(\Phi, \boldsymbol{V})=\left\{\boldsymbol{\alpha} \in \Omega^{\bullet}(\Phi, \boldsymbol{V}) \mid R_{\gamma}^{\star} \boldsymbol{\alpha}=\right.$ $\left.\rho(\gamma)^{-1} \boldsymbol{\alpha}\right\}$. The infinitesimal version of the equivariance property is $\boldsymbol{L}_{\chi^{v}} \boldsymbol{\alpha}=-\rho_{*}(\chi) \boldsymbol{\alpha}$. The subspace of invariant forms is $\Omega_{\text {inv }}^{\bullet}(\Phi, \boldsymbol{V})=\left\{\boldsymbol{\alpha} \in \Omega^{\bullet}(\Phi, \boldsymbol{V}) \mid R_{\gamma}^{\star} \boldsymbol{\alpha}=\boldsymbol{\alpha}\right\}$, infinitesimally $\boldsymbol{L}_{\chi^{v}} \boldsymbol{\alpha}=0$.

The space of horizontal forms is

$$
\Omega_{\mathrm{hor}}^{\bullet}(\Phi)=\left\{\boldsymbol{\alpha} \in \Omega^{\bullet}(\Phi) \mid \iota_{\chi^{v}} \boldsymbol{\alpha}=0\right\}
$$

A form which is both horizontal and equivariant is said tensorial:

$$
\Omega_{\text {tens }}^{\bullet}(\Phi, \boldsymbol{V})=\left\{\boldsymbol{\alpha} \in \Omega^{\bullet}(\Phi, \boldsymbol{V}) \mid R_{\gamma}^{\star} \boldsymbol{\alpha}=\rho(\gamma)^{-1} \boldsymbol{\alpha}, \quad \& \quad \iota_{\chi^{v}} \boldsymbol{\alpha}=0\right\} .
$$

Clearly, $\Omega_{\text {tens }}^{0}(\Phi, \boldsymbol{V})=\Omega_{\mathrm{eq}}^{0}(\Phi, \boldsymbol{V})$. Finally, basic forms are both horizontal and invariant:

$$
\Omega_{\text {basic }}^{\bullet}(\Phi)=\left\{\boldsymbol{\alpha} \in \Omega^{\bullet}(\Phi) \mid R_{\gamma}^{\star} \boldsymbol{\alpha}=\boldsymbol{\alpha}, \quad \& \quad \iota_{\chi^{v}} \boldsymbol{\alpha}=0\right\} .
$$

Alternatively, basic forms are defined as $\operatorname{Im}\left(\pi^{\star}\right)$, that is:

$$
\Omega_{\text {basic }}^{\bullet}(\Phi)=\left\{\boldsymbol{\alpha} \in \Omega^{\bullet}(\Phi) \mid \exists \boldsymbol{\beta} \in \Omega^{\bullet}(\mathcal{M}) \text { s.t. } \boldsymbol{\alpha}=\pi^{\star} \boldsymbol{\beta}\right\} \text {. }
$$

Remark that the form analogue of $\Gamma_{\mathcal{H}}(T \Phi)$ - that projects to well defined vector fields in $\Gamma(M)$ - is not $\Omega_{\text {inv }}^{\bullet}(\Phi)$ but $\Omega_{\text {basic }}^{\bullet}(\Phi)$, only the latter projects to well-defined forms in $\Omega^{\bullet}(\mathcal{M})$. From $\left[\boldsymbol{d}, \pi^{\star}\right]=0$ follows that $\left(\Omega_{\text {basic }}^{\bullet}(\Phi), \boldsymbol{d}\right)$ is a subcomplex of the de Rham complex: the basic subcomplex. The associated cohomology, isomorphic to the cohomology of $\left(\Omega^{\bullet}(\mathcal{M}), \boldsymbol{d}\right)$, is by definition the equivariant cohomology of $\Phi$. As we advertised, the main preoccupation of this paper, in relation to the boundary problem in gauge theory, will be to consider ways to construct basic forms.

Notice that, as in the finite dimensional case, $\Omega_{\mathrm{eq}}^{0}(\Phi, \boldsymbol{V}) \simeq \Gamma(\boldsymbol{E})$ where $\boldsymbol{E} \rightarrow \mathcal{M}$ is an associated bundle to $\Phi$ built via the representation $(\boldsymbol{V}, \rho)$ in the usual way: defining a right action of $\mathcal{H}$ on $\Phi \times \boldsymbol{V}$ by $(\phi, \gamma) \mapsto\left(\phi^{\gamma}, \rho(\gamma)^{-1} \boldsymbol{v}\right)$, and considering it is an equivalence relation $\sim$ between pairs, the associated bundle $\boldsymbol{E}$ is defined as the space of equivalence classes; $\boldsymbol{E}=\Phi \times_{\mathcal{H}} \boldsymbol{V}:=\Phi \times \boldsymbol{V} / \sim$. The 1:1 correspondance between $\boldsymbol{s} \in \Gamma(\boldsymbol{E})$ and $\boldsymbol{\varphi} \in \Omega_{\mathrm{eq}}^{0}(\Phi, \boldsymbol{V})$ is, $\boldsymbol{s}([\phi])=[\phi, \boldsymbol{\varphi}(\phi)]$.

\footnotetext{
${ }^{6}$ The vector space of derivation $\operatorname{Der}(\mathrm{A})$ of an algebra $\mathrm{A}$ is a Lie algebra under the graded bracket $\left[d_{1}, d_{2}\right]:=d_{1} \circ d_{2}-(-)^{\left|d_{1}\right| \cdot\left|d_{2}\right|} d_{2} \circ d_{1}$.
} 
It is perhaps interesting to remark that, in view of this standard construction, the base space $\mathcal{M}$ of $\Phi$ is itself a bundle associated with the $\mathcal{H}$-principal bundle $\mathcal{A} \rightarrow \mathcal{A} / \mathcal{H}$. Indeed $\Gamma(E)$ is a $\mathcal{H}$-space, and considering the classes under the right action of $\mathcal{H}$ on $\Phi=\mathcal{A} \times \Gamma(E)$ one obtains the quotient space $\mathcal{M}=\mathcal{A} \times{ }_{\mathcal{H}} \Gamma(E):=\mathcal{A} \times \Gamma(E) / \sim$. Therefore, the field space is a doubly fibered space, $\Phi \rightarrow \mathcal{M} \rightarrow \mathcal{A} / \mathcal{H}$. The space of sections of $\mathcal{M}$, $\Gamma(\mathcal{M}):=\{s: \mathcal{A} / \mathcal{H} \rightarrow \mathcal{M},[A] \mapsto s([A])=[A, \varphi]\}$, is then isomorphic to $\Omega_{\text {eq }}^{0}(\mathcal{A}, \Gamma(E)):=$ $\left\{\hat{\boldsymbol{s}}: \mathcal{A} \rightarrow \Gamma(E) \mid R_{\gamma}^{\star} \hat{\boldsymbol{s}}=\rho(\gamma)^{-1} \hat{\boldsymbol{s}}\right\}$. One would remark that the covariant derivative map of a section $\varphi \in \Gamma(E), A \mapsto\left\{\iota_{X} D \varphi\right\}(A)=\iota_{X} D^{A} \varphi$ - for any vector field $X \in \Gamma(T \mathcal{P})^{7}$ is an element of this space since $R_{\gamma}^{\star} D^{A} \varphi=\rho(\gamma)^{-1} D^{A} \varphi$. This shows the centrality of the role payed by the connection space $\mathcal{A}$, which is reminiscent of the fact that a $H$-principal bundle $\mathcal{P}$ 'controls' all associated bundles built via representations of $H$ (or $H$-spaces more generally).

In the construction above, one can replace representations $(\rho, \boldsymbol{V})$ by 1-cocycles for the action of $\mathcal{H}$, i.e. a smooth map $C: \Phi \times \mathcal{H} \rightarrow G,(\phi, \gamma) \mapsto C(\phi, \gamma)$, satisfying the relation $C\left(\phi, \gamma \gamma^{\prime}\right)=C(\phi, \gamma) C\left(\phi^{\gamma}, \gamma^{\prime}\right)$, and $\boldsymbol{V}$ a $G$-space. Then, we have a well-defined right action of $\mathcal{H}$ on $\Phi \times \boldsymbol{V}$ twisted by a cocycle $(\phi, \boldsymbol{v}) \mapsto\left(\phi^{\gamma}, C(\phi, \gamma)^{-1} \boldsymbol{v}\right)$. One thus defines twisted associated bundles as the spaces of equivalence classes under this action, $\tilde{\boldsymbol{E}}:=\Phi \times_{\mathcal{C}} \boldsymbol{V}$, whose spaces of sections are isomorphic to the space of $C$-equivariant $\boldsymbol{V}$-valued maps on $\Phi, \Gamma(\tilde{\boldsymbol{E}}) \simeq$ $\Omega_{\mathrm{eq}}^{0}(\Phi, C):=\left\{\boldsymbol{\varphi}: \Phi \rightarrow \boldsymbol{V} \mid \boldsymbol{\varphi}\left(\phi^{\gamma}\right)=C(\phi, \gamma)^{-1} \boldsymbol{\varphi}(\phi)\right\}$. More generally, one has the welldefined spaces of $C$-equivariant forms $\Omega_{\mathrm{eq}}^{\bullet}(\Phi, C):=\left\{\boldsymbol{\alpha} \in \Omega^{\bullet}(\Phi, \boldsymbol{V}) \mid R_{\gamma}^{\star} \boldsymbol{\alpha}=C(, \gamma)^{-1} \boldsymbol{\alpha}\right\}$, and of $C$-tensorial forms $\Omega_{\text {tens }}^{\bullet}(\Phi, C):=\left\{\boldsymbol{\alpha} \in \Omega_{\text {eq }}^{\bullet}(\Phi, C) \mid \iota_{\chi^{v}} \boldsymbol{\alpha}=0\right\}$.

If the covariant differentiation of standard equivariant forms necessitates to endow $\Phi$ with variational Ehresmann (principal) connections, the covariant differentiation of $C$ equivariant forms requires the introduction of twisted connections which are a generalisation of the latter. See section 2.2 below. We refer to [47] for an in depth exposition of the basics of the twisted geometry on bundles, and to section 2.3 of [33] for a nutshell presentation.

Gauge transformations. The local representative of a form $\boldsymbol{\alpha} \in \Omega^{\bullet}(\Phi)$ as seen through a section $\boldsymbol{\sigma}: \mathcal{U} \rightarrow \Phi$ is $\boldsymbol{a}:=\boldsymbol{\sigma}^{\star} \boldsymbol{\alpha}$, so that $\boldsymbol{a}_{\mid[\phi]}\left(\boldsymbol{X}_{[\phi]}\right)=\boldsymbol{\alpha}_{\mid \boldsymbol{\sigma}([\phi])}\left(\boldsymbol{\sigma}_{\star} \boldsymbol{X}_{[\phi]}\right)$. In view of (2.3), two local representatives $\boldsymbol{a}=\boldsymbol{\sigma}^{\star} \boldsymbol{\alpha}$ and $\boldsymbol{a}^{\prime}=\boldsymbol{\sigma}^{\prime \star} \boldsymbol{\alpha}$ satisfy gluing relations on $\mathcal{U} \cap \mathcal{U}^{\prime} \neq$ $\emptyset$ - also called passive gauge transformations - controlled by the $\mathcal{H}$-equivariance and verticality properties of $\boldsymbol{\alpha}$. In particular, it is clear that if $\boldsymbol{\alpha}$ is tensorial, the gluing relations of its local representatives are simply $\boldsymbol{a}^{\prime}=\rho(\boldsymbol{h})^{-1} \boldsymbol{a}$. Similarly, if $\boldsymbol{\alpha}$ is $C$-tensorial, the gluing relations of its local representatives are simply $\boldsymbol{a}^{\prime}=C(\boldsymbol{\sigma}, \boldsymbol{h})^{-1} \boldsymbol{a}$. The local representatives of a basic form have trivial gluing relations: $\boldsymbol{a}^{\prime}=\boldsymbol{a}$. Hence again the interest of such forms. We will not make use of this notion of passive, or $[\phi]$-dependent, gauge transformation, as we aim to work primarily on $\Phi$ rather than on $\mathcal{M}$. But we articulate it if only to clearly distinguish it from the notion of gauge transformation on $\Phi$ that will be of interest from now on.

The active, $\phi$-dependent, gauge transformation of a form $\boldsymbol{\alpha} \in \Omega^{\bullet}(\Phi)$ is defined by the action of $\operatorname{Aut}_{v}(\mathcal{A}) \simeq \mathcal{H}$ via $\boldsymbol{\alpha}^{\gamma}:=\Psi^{\star} \boldsymbol{\alpha}$. So that, $\boldsymbol{\alpha}_{\mid \phi}^{\gamma}\left(\boldsymbol{X}_{\phi}\right)=\boldsymbol{\alpha}_{\mid \boldsymbol{\Psi}(\phi)}\left(\boldsymbol{\Psi}_{\star} \boldsymbol{X}_{\phi}\right)$. By (2.4),

\footnotetext{
${ }^{7}$ The covariant derivative map would be $D: \mathcal{A} \times \Gamma(E) \rightarrow \Omega_{\text {tens }}^{1}(\mathcal{P}, V),(A, \varphi) \mapsto D^{A} \varphi$. So that, for any vector field $X \in \Gamma(T \mathcal{P})$, we have the map $\iota_{X} D: \mathcal{A} \times \Gamma(E) \rightarrow \Gamma(E),(A, \varphi) \mapsto \iota_{X} D^{A} \varphi$.
} 
the $\mathcal{H}$-gauge transformation of a variational form is thus controlled by its $\mathcal{H}$-equivariance and verticality properties. It follows immediately that the $\mathcal{H}$-gauge transformation of a tensorial form is $\boldsymbol{\alpha}^{\gamma}=\rho(\boldsymbol{\gamma})^{-1} \boldsymbol{\alpha}$, or infinitesimally $\boldsymbol{L}_{\chi^{v}} \boldsymbol{\alpha}=-\rho_{*}(\boldsymbol{\chi}) \boldsymbol{\alpha}$, with $\boldsymbol{\chi} \in$ Lie $\mathcal{H}$. In the same way, $C$-tensorial forms $\mathcal{H}$-transform as $\boldsymbol{\alpha}^{\gamma}=C(, \boldsymbol{\gamma})^{-1} \boldsymbol{\alpha}$, or infinitesimally $\boldsymbol{L}_{\boldsymbol{\chi}^{v}} \boldsymbol{\alpha}=-\left.\frac{d}{d \tau} C(, \exp \tau \boldsymbol{\chi})\right|_{\tau=0} \boldsymbol{\alpha}$. Basic forms are $\mathcal{H}$-invariant, $\boldsymbol{\alpha}^{\gamma}=\boldsymbol{\alpha}$ or $\boldsymbol{L}_{\boldsymbol{\chi}^{v}} \boldsymbol{\alpha}=0$.

Basis 1-forms. As a relevant illustration, consider $\boldsymbol{d} \phi=\{\boldsymbol{d} A, \boldsymbol{d} \varphi\} \in \Omega^{1}(\phi)$ the basis for variational forms on $\Phi$. Its verticality property reproduces, by definition, the infinitesimal gauge transformations of the fields,

$$
\boldsymbol{d} \phi_{\mid \phi}\left(\chi_{\phi}^{v}\right)=\left(\boldsymbol{d} A_{\mid A}\left(\chi_{A}^{v}\right), \boldsymbol{d} \varphi_{\mid \varphi}\left(\chi_{\phi}^{v}\right)\right)=\left(D^{A} \chi,-\rho_{*}(\chi) \varphi\right)=: \delta_{\chi} \phi
$$

The last equality introduces a convenient notation. We thus find that on $\Gamma(V \Phi)$, its $\mathcal{H}$-equivariance property is, $R_{\gamma}^{\star} \boldsymbol{d} \phi_{\mid \phi^{\gamma}}\left(\chi^{v}\right)=\boldsymbol{d} \phi_{\mid A^{\gamma}}\left(R_{\gamma \star} \chi_{\phi}^{v}\right)=$ $\boldsymbol{d} \phi_{\mid \phi^{\gamma}}\left(\gamma^{-1} \chi \gamma\right)_{\phi^{\gamma}}^{v}=\left(D^{A^{\gamma}}\left(\gamma^{-1} \chi \gamma\right),-\rho_{*}\left(\gamma^{-1} \chi \gamma\right) \varphi^{\gamma}\right)=\left(\gamma^{-1}\left(D^{A} \chi\right) \gamma,-\rho(\gamma)^{-1} \rho_{*}(\chi) \varphi\right)=$ $\left(\gamma^{-1} \boldsymbol{d} A_{\mid A}\left(\chi_{A}^{v}\right) \gamma, \rho(\gamma)^{-1} \boldsymbol{d} \varphi_{\mid \phi}\left(\chi_{\varphi}^{v}\right)\right)$, by (2.5). Introducing the notation $\rho:=(\operatorname{Ad}, \rho)$, we write the equivariance compactly as

$$
R_{\gamma}^{\star} \boldsymbol{d} \phi=\rho(\gamma)^{-1} \boldsymbol{d} \phi:=\left(\gamma^{-1} \boldsymbol{d} A \gamma, \rho(\gamma)^{-1} \boldsymbol{d} \varphi\right) .
$$

We require this to hold on $\Gamma(T \Phi)$, so that $\boldsymbol{d} \phi \in \Omega_{\mathrm{eq}}^{1}(\Phi, T \Phi)$. By (2.4), it is now easy to find the $\mathcal{H}$-gauge transformation of $\boldsymbol{d} \phi$ to be,

$$
\begin{aligned}
\boldsymbol{d} \phi_{\mid \phi}^{\gamma}\left(\boldsymbol{X}_{\phi}\right) & :=\left(\boldsymbol{\Psi}^{\star} \boldsymbol{d} \phi\right)_{\mid \phi}\left(\boldsymbol{X}_{\phi}\right) \\
& =\boldsymbol{d} \phi_{\mid \phi^{\gamma}}\left(\boldsymbol{\Psi}_{\star} \boldsymbol{X}_{\phi}\right), \\
& =\boldsymbol{d} \phi_{\mid \phi^{\gamma}}\left(R_{\gamma(\phi) \star}\left[\boldsymbol{X}_{\phi}+\left\{\boldsymbol{d} \boldsymbol{\gamma} \boldsymbol{\gamma}_{\mid \phi}^{-1}\left(\boldsymbol{X}_{\phi}\right)\right\}_{\phi}^{v}\right]\right) \\
& =\left(R_{\gamma(\phi)}^{\star} \boldsymbol{d} \phi_{\mid \phi^{\gamma}}\right)\left(\boldsymbol{X}_{\phi}+\left\{\boldsymbol{d} \boldsymbol{\gamma} \gamma_{\mid \phi}^{-1}\left(\boldsymbol{X}_{\phi}\right)\right\}_{\phi}^{v}\right), \\
& =\rho(\gamma(\phi))^{-1} \boldsymbol{d} \phi_{\mid \phi}\left(\boldsymbol{X}_{\phi}+\left\{\boldsymbol{d} \boldsymbol{\gamma} \gamma_{\mid \phi}^{-1}\left(\boldsymbol{X}_{\phi}\right)\right\}_{\phi}^{v}\right) \\
& =\rho(\gamma(\phi))^{-1}\left(\boldsymbol{d} \phi_{\mid \phi}\left(\boldsymbol{X}_{\phi}\right)+\delta_{\boldsymbol{d} \gamma \gamma_{\mid \phi}^{-1}\left(\boldsymbol{X}_{\phi}\right)} \phi\right) .
\end{aligned}
$$

That is, for $\gamma \in \mathcal{H}$,

$$
\boldsymbol{d} \phi^{\gamma}=\rho(\boldsymbol{\gamma})^{-1}\left(\boldsymbol{d} \phi+\delta_{\boldsymbol{d} \gamma \gamma^{-1}} \phi\right)=\left\{\begin{array}{l}
\boldsymbol{d} A^{\gamma}=\boldsymbol{\gamma}^{-1}\left(\boldsymbol{d} A+D^{A}\left\{\boldsymbol{d} \boldsymbol{\gamma} \boldsymbol{\gamma}^{-1}\right\}\right) \boldsymbol{\gamma} \\
\boldsymbol{d} \varphi^{\gamma}=\rho(\boldsymbol{\gamma})^{-1}\left(\boldsymbol{d} \varphi-\rho_{*}\left(\boldsymbol{d} \boldsymbol{\gamma} \boldsymbol{\gamma}^{-1}\right) \varphi\right)
\end{array}\right.
$$

The infinitesimal version is easily read from the above, by $\gamma \rightarrow \chi \in$ Lie $\mathcal{H}$ and keeping only the linear terms, and of course seen to match the result obtained by,

$$
\begin{aligned}
\boldsymbol{L}_{\chi^{v}} \boldsymbol{d} \phi=\left(\iota_{\chi^{v}} \boldsymbol{d}+\boldsymbol{d} \iota_{\chi^{v}}\right) \boldsymbol{d} \phi=\boldsymbol{d} \delta_{\chi} \phi & =\boldsymbol{d}\left(D^{A} \boldsymbol{\chi},-\rho_{*}(\boldsymbol{\chi}) \varphi\right), \\
& =\left([\boldsymbol{d} A, \boldsymbol{\chi}]+D^{A}(\boldsymbol{d} \boldsymbol{\chi}),-\rho_{*}(\boldsymbol{\chi}) \boldsymbol{d} \varphi-\rho_{*}(\boldsymbol{d} \boldsymbol{\chi}) \varphi\right) .
\end{aligned}
$$


In term of the basis $\boldsymbol{d} \phi$, a generic variational form $\boldsymbol{\alpha} \in \Omega^{\bullet}(\Phi)$ will be written $\boldsymbol{\alpha}_{\mid \phi}=$ $\alpha\left(\wedge^{\bullet} \boldsymbol{d} \phi_{\mid \phi} ; \phi\right)$, or simply $\boldsymbol{\alpha}=\alpha\left(\wedge^{\bullet} \boldsymbol{d} \phi ; \phi\right)$, where $\alpha($; ) is alternating multilinear in the first arguments and its second argument denotes the functional dependence of $\boldsymbol{\alpha}$ on $\phi$. So, in concrete situations, given such an expression for $\boldsymbol{\alpha}$ and eq. (2.7), it is possible to compute algebraically its $\mathcal{H}$-gauge transformation by $\boldsymbol{\alpha}^{\gamma}=\alpha\left(\wedge^{\bullet} \boldsymbol{d} \phi^{\gamma} ; \phi^{\gamma}\right)$, thereby cross-checking the geometric result obtained via $\boldsymbol{\alpha}^{\gamma}=\boldsymbol{\Psi}^{\star} \boldsymbol{\alpha}$.

Curvature and covariant derivative maps. We provide further illustrations by iterating the process on two quantities relevant for what follows, that we consider in turn.

- Curvature map: consider the $\mathcal{H}$-equivariant curvature map $F: \Phi \rightarrow \Omega_{\text {tens }}^{2}(\mathcal{P}, \operatorname{Lie} H)$, $(A, \varphi) \mapsto F(A)=d A+\frac{1}{2}[A, A]$, s.t. $R_{\gamma}^{\star} F=\gamma^{-1} F \gamma$. Given $\boldsymbol{X} \in \Gamma(T \mathcal{A})$ with flow $f_{\tau}$, we have:

$$
\begin{aligned}
\boldsymbol{d} F_{\mid \phi}\left(\boldsymbol{X}_{\phi}\right) & =\boldsymbol{X}(F)(\phi)=\left.\frac{d}{d \tau} F\left(f_{\tau}(\phi)\right)\right|_{\tau=0} \\
& =\frac{d}{d \tau} d f_{\tau}(A)+\left.\frac{1}{2}\left[f_{\tau}(A), f_{\tau}(A)\right]\right|_{\tau=0} \\
& =D^{A}\left(\left.\frac{d}{d \tau} f_{\tau}(A)\right|_{\tau=0}\right) \\
& =D^{A}\left(\boldsymbol{d} A_{\mid \phi}\left(\boldsymbol{X}_{\phi}\right)\right)
\end{aligned}
$$

which is simply $\boldsymbol{d} F=D(\boldsymbol{d} A)$.

Then, the verticality property of $\boldsymbol{d} F$ reproduces the infinitesimal $\mathcal{H}$ transformation of $F$ :

$$
\boldsymbol{d} F_{\mid \phi}\left(\chi_{\phi}^{v}\right)=D^{A}\left(\boldsymbol{d} A_{\mid \phi}\left(\chi_{\phi}^{v}\right)\right)=D^{A}\left(D^{A} \chi\right)=[F(A), \chi], \quad \text { or } \quad \iota_{\chi^{v}} \boldsymbol{d} F=[F, \chi] .
$$

Its $\mathcal{H}$-equivariance is

$$
\begin{aligned}
R_{\gamma}^{\star} \boldsymbol{d} F_{\mid \phi^{\gamma}}\left(\boldsymbol{X}_{\phi}\right) & =\boldsymbol{d} F_{\phi^{\gamma}}\left(R_{\gamma \star} \boldsymbol{X}_{\phi}\right)=D^{A^{\gamma}}\left(\boldsymbol{d} \phi_{\mid \phi^{\gamma}}\left(R_{\gamma \star} \boldsymbol{X}_{\phi}\right)\right)=D^{A^{\gamma}}\left(R_{\gamma}^{\star} \boldsymbol{d} A_{\mid \phi^{\gamma}}\left(\boldsymbol{X}_{\phi}\right)\right), \\
& =D^{A^{\gamma}}\left(\gamma^{-1} \boldsymbol{d} A_{\mid \phi}\left(\boldsymbol{X}_{\phi}\right) \gamma\right)=\gamma^{-1} D^{A}\left(\boldsymbol{d} A_{\mid \phi}\left(\boldsymbol{X}_{\phi}\right)\right) \gamma=\gamma^{-1} \boldsymbol{d} F_{\mid \phi}\left(\boldsymbol{X}_{\phi}\right) \gamma, \\
\text { or } \quad R_{\gamma}^{\star} \boldsymbol{d} F & =\gamma^{-1} \boldsymbol{d} F \gamma .
\end{aligned}
$$

This result is also found simply from the fact that pullbacks and $\boldsymbol{d}$ commute, so that $R_{\gamma}^{\star} \boldsymbol{d} F=\boldsymbol{d} R_{\gamma}^{\star} F=\gamma^{-1} \boldsymbol{d} F \gamma$. From (2.10)-(2.11) and (2.4), the $\mathcal{H}$-gauge transformation of $\boldsymbol{d F}$ is found to be

$$
\begin{aligned}
\boldsymbol{d} F_{\mid \phi}^{\gamma}\left(\boldsymbol{X}_{\phi}\right) & :=\left(\Psi^{\star} \boldsymbol{d} F\right)_{\mid \phi}\left(\boldsymbol{X}_{\phi}\right)=\boldsymbol{d} F_{\mid \phi^{\gamma}}\left(\boldsymbol{\Psi}_{\star} \boldsymbol{X}_{\phi}\right), \\
& =\boldsymbol{d} F_{\mid \phi^{\gamma}}\left(R_{\gamma(\phi) \star}\left[\boldsymbol{X}_{\phi}+\left\{\boldsymbol{d} \boldsymbol{\gamma} \boldsymbol{\gamma}_{\mid \phi}^{-1}\left(\boldsymbol{X}_{\phi}\right)\right\}_{\phi}^{v}\right]\right) \\
& =\left(R_{\gamma(\phi)}^{\star} \boldsymbol{d} F_{\mid \phi^{\gamma}}\right)\left(\boldsymbol{X}_{\phi}+\left\{\boldsymbol{d} \boldsymbol{\gamma} \boldsymbol{\gamma}_{\mid \phi}^{-1}\left(\boldsymbol{X}_{\phi}\right)\right\}_{\phi}^{v}\right), \\
& =\gamma(\phi)^{-1} \boldsymbol{d} F_{\mid \phi}\left(\boldsymbol{X}_{\phi}+\left\{\boldsymbol{d} \boldsymbol{\gamma} \boldsymbol{\gamma}_{\mid \phi}^{-1}\left(\boldsymbol{X}_{\phi}\right)\right\}_{\phi}^{v}\right) \gamma(\phi) \\
& =\gamma(\phi)^{-1}\left(\boldsymbol{d} F_{\mid \phi}\left(\boldsymbol{X}_{\phi}\right)+\left[F(\phi), \boldsymbol{d} \boldsymbol{\gamma} \boldsymbol{\gamma}_{\mid \phi}^{-1}\left(\boldsymbol{X}_{\phi}\right)\right]\right) \gamma(\phi), \\
\text { that is } \boldsymbol{d} F^{\gamma} & =\gamma^{-1}\left(\boldsymbol{d} A+\left[F, \boldsymbol{d} \boldsymbol{\gamma} \boldsymbol{\gamma}^{-1}\right]\right) \boldsymbol{\gamma}
\end{aligned}
$$


This can also be checked algebraically, using (2.7) in $\boldsymbol{d} F^{\gamma}=D^{A^{\gamma}}\left(\boldsymbol{d} A^{\gamma}\right)$.

- Covariant derivative map: let us consider the $\mathcal{H}$-equivariant covariant derivative map $D: \Phi \rightarrow \Omega_{\text {tens }}^{1}(\mathcal{P}, V), \phi=(A, \varphi) \mapsto D^{A} \varphi=d A+\rho_{*}(A) \varphi$, s.t. $R_{\gamma}^{\star} D=\rho(\gamma)^{-1} D$. Given $\boldsymbol{X} \in \Gamma(T \Phi)$ with flow $f_{\tau}$, we get:

$$
\begin{aligned}
\boldsymbol{d} D_{\mid \phi}\left(\boldsymbol{X}_{\phi}\right)=\boldsymbol{X}(D)(\phi) & =\left.\frac{d}{d \tau} D^{f_{\tau}(A)} f_{\tau}(\varphi)\right|_{\tau=0} \\
& =\frac{d}{d \tau} d f_{\tau}(\varphi)+\left.\rho_{*}\left(f_{\tau}(A)\right) f_{\tau}(\varphi)\right|_{\tau=0} \\
& =D^{A}\left(\left.\frac{d}{d \tau} f_{\tau}(\varphi)\right|_{\tau=0}\right)+\rho_{*}\left(\left.\frac{d}{d \tau} f_{\tau}(A)\right|_{\tau=0}\right) \varphi \\
& =D^{A}\left(\boldsymbol{d} \varphi_{\mid \phi}\left(\boldsymbol{X}_{\phi}\right)\right)+\rho_{*}\left(\boldsymbol{d} A_{\mid \phi}\left(\boldsymbol{X}_{\phi}\right)\right) \varphi
\end{aligned}
$$

which we can write $\boldsymbol{d} D^{A} \varphi=D^{A}(\boldsymbol{d} \varphi)+\rho_{*}(\boldsymbol{d} A) \varphi$,

by a slight abuse of notation (on the left-hand side) to make the expression more transparent. As one expects, the verticality property of $\boldsymbol{d} D^{A} \varphi$ reproduces the infinitesimal $\mathcal{H}$-transformation of $D^{A} \varphi$ :

$$
\begin{aligned}
\boldsymbol{d} D^{A} \varphi_{\mid \phi}\left(\chi_{\phi}^{v}\right) & =D^{A}\left(\boldsymbol{d} \varphi_{\mid \phi}\left(\chi_{\phi}^{v}\right)\right)+\rho_{*}\left(\boldsymbol{d} A_{\mid \phi}\left(\chi_{\phi}^{v}\right)\right) \varphi \\
& =D^{A}\left(-\rho_{*}(\chi) \varphi\right)+\rho_{*}\left(D^{A} \chi\right) \varphi=-\rho_{*}(\chi) D^{A} \varphi .
\end{aligned}
$$

As for the $\mathcal{H}$-equivariance, we have

$$
\begin{aligned}
R_{\gamma}^{\star} \boldsymbol{d} D^{A} \varphi_{\mid \phi^{\gamma}}\left(\boldsymbol{X}_{\phi}\right) & =\boldsymbol{d} D^{A} \varphi_{\mid \phi^{\gamma}}\left(R_{\gamma \star} \boldsymbol{X}_{\phi}\right) \\
& =D^{A^{\gamma}}\left(\boldsymbol{d} \varphi_{\mid \phi^{\gamma}}\left(R_{\gamma \star} \boldsymbol{X}_{\phi}\right)\right)+\rho_{*}\left(\boldsymbol{d} A_{\mid \phi^{\gamma}}\left(R_{\gamma \star} \boldsymbol{X}_{\phi}\right)\right) \varphi^{\gamma}, \\
& =D^{A^{\gamma}}\left(R_{\gamma}^{\star} \boldsymbol{d} \varphi_{\mid \phi^{\gamma}}\left(\boldsymbol{X}_{\phi}\right)\right)+\rho_{*}\left(R_{\gamma}^{\star} \boldsymbol{d} A_{\mid \phi^{\gamma}}\left(\boldsymbol{X}_{\phi}\right)\right) \varphi^{\gamma}, \\
& =D^{A^{\gamma}}\left(\rho(\gamma)^{-1} \boldsymbol{d} \varphi_{\mid \phi}\left(\boldsymbol{X}_{\phi}\right)\right)+\rho_{*}\left(\gamma^{-1} \boldsymbol{d} A_{\mid \phi}\left(\boldsymbol{X}_{\phi}\right) \gamma\right) \varphi^{\gamma}, \\
& =\rho(\gamma)^{-1}\left(D^{A}\left(\boldsymbol{d} \varphi_{\mid \phi}\left(\boldsymbol{X}_{\phi}\right)\right)+\rho_{*}\left(\boldsymbol{d} A_{\mid \phi}\left(\boldsymbol{X}_{\phi}\right)\right) \varphi\right),
\end{aligned}
$$

which is $\quad R_{\gamma}^{\star} d D^{A} \varphi=\rho(\gamma)^{-1} \boldsymbol{d} D^{A} \varphi$.

Which also results from $\left[R_{\gamma}^{\star}, \boldsymbol{d}\right]=0$. Again, by (2.14)-(2.15) and (2.4) we get the $\mathcal{H}$-gauge transformation:

$$
\begin{aligned}
\left(\boldsymbol{d} D^{A} \varphi\right)^{\gamma}(\boldsymbol{X}) & =\left(\boldsymbol{\Psi}^{\star} \boldsymbol{d} D^{A} \varphi\right)(\boldsymbol{X})=\boldsymbol{d} D^{A} \varphi\left(\boldsymbol{\Psi}_{\star} \boldsymbol{X}\right)=\ldots \\
\Rightarrow\left(\boldsymbol{d} D^{A} \varphi\right)^{\gamma} & =\rho(\boldsymbol{\gamma})^{-1}\left(\boldsymbol{d} D^{A} \varphi-\rho_{*}\left(\boldsymbol{d} \boldsymbol{\gamma} \boldsymbol{\gamma}^{-1}\right) D^{A} \varphi\right) .
\end{aligned}
$$

Lagrangians. A gauge theory is specified by a choice of Lagrangian functional $L: \Phi \rightarrow$ $\Omega^{n}(\mathcal{P}), \phi:=(A, \varphi) \mapsto L(\phi)=L(A, \varphi)$, with $n=\operatorname{dim} M$, whose $\mathcal{H}$-equivariance is usually prescribed. Said otherwise $L \in \Omega_{\mathrm{eq}}^{0}(\Phi)$, so that a Lagrangian can be seen as a section of some bundle associated with $\Phi$. We consider the following three cases.

- Case 1: invariant gauge theories. The Lagrangian strictly satisfies the gauge principle, $R_{\gamma}^{\star} L=L$, so $L \in \Omega_{\text {basic }}^{0}(\Phi)$. This means that $\exists \bar{L} \in \Omega^{0}(\mathcal{M})$ s.t. $L=\pi^{\star} \bar{L}$. 
It also means that $L$ is the section of the bundle associated with $\Phi$ via the trivial representation. So is $Z:=\exp i \int L \in \Omega_{\text {basic }}(\Phi, \mathbb{C})$. Thus, they are both $\mathcal{H}$-invariant: $L^{\gamma}=L$ and $Z^{\gamma}=Z$. The Yang-Mills Lagrangian is an example.

- Case 2: non-invariant gauge theories transforming via representations. The Lagrangian has $\mathcal{H}$-equivariance $R_{\gamma}^{\star} L=L+\tilde{\rho}(\gamma)$, so that $R_{\gamma}^{\star} Z=\rho(\gamma)^{-1} Z$, where $\rho(\gamma):=\exp -i \int \tilde{\rho}(\gamma)$ is a representation of $\mathcal{H}$ on $\mathbb{C}$. We have $L, Z \in \Omega_{\mathrm{eq}}^{0}(\Phi)$ (by a slight abuse of notation in the case of $L$ ), they are sections of standard bundles associated with $\Phi$. So, their $\mathcal{H}$-gauge transformations are $L^{\gamma}:=\Psi^{\star} L=L+\tilde{\rho}(\boldsymbol{\gamma})$ and $Z^{\gamma}=\rho(\gamma)^{-1} Z$. A simple example is the 3D Abelian Chern-Simons Lagrangian.

- Case 3: non-invariant gauge theories transforming via cocycles. The Lagrangian has $\mathcal{H}$-equivariance $R_{\gamma}^{\star} L=L+c(, \gamma)$, so that $R_{\gamma}^{\star} Z=C(, \gamma)^{-1} Z$, where $C(, \gamma):=$ $\exp -i \int c(, \gamma)$ is a 1 -cocycle for the action of $\mathcal{H}$ on $\mathbb{C}$. We have $L, Z \in \Omega_{\text {eq }}^{0}(\Phi, C)$ (by a slight abuse of notation in the case of $L$ ), they are sections of twisted bundles associated to $\Phi$. So their $\mathcal{H}$-gauge transformations are $L^{\gamma}:=\boldsymbol{\Psi}^{\star} L=L+c(, \gamma)$ and $Z^{\gamma}=C(, \gamma)^{-1} Z$. The Lagrangians of 3D non-Abelian Chern-Simons and massive Yang-Mills theories illustrate this case.

In the next two sections, we discuss structures that are a priori non-canonical on the bundle $\Phi$. In the first, we elaborate around the standard notion of Ehresmann - or principal - variational connection, and then briefly comment on a generalisation called twisted variational connection. In the next, we give a nutshell presentation of a tool to build basic forms, known as the dressing field method. The material exposed in both these sections will be used to address the problem of boundaries in the presymplectic structure of invariant gauge theories (case 1 above) in section 4, where we will only make passing comments on the case of non-invariant theories (cases 2 and 3 ).

\subsection{Variational connections on field space}

\subsubsection{Variational Ehresmann connections}

As mentioned above, to split the SES (2.2) and to define a notion of horizontality, one needs to introduce a choice of Ehresmann connection on $\Phi$. Such a variational connection $\boldsymbol{\omega} \in \Omega_{\mathrm{eq}}^{1}(\Phi$, Lie $\mathcal{H})$ is by definition s.t.

$$
\begin{aligned}
R_{\gamma}^{\star} \boldsymbol{\omega} & =\gamma^{-1} \boldsymbol{\omega} \gamma, \\
\boldsymbol{\omega}\left(\chi^{v}\right) & =\chi \in \text { Lie } \mathcal{H} .
\end{aligned}
$$

It is not unique, and from the definition follows that the space of variational connections is affine and modeled on the vector space $\Omega_{\text {tens }}^{1}(\Phi$, Lie $\mathcal{H})$. That is, for $\beta \in \Omega_{\text {tens }}^{1}(\Phi$, Lie $\mathcal{H})$, we have that $\boldsymbol{\omega}^{\prime}=\boldsymbol{\omega}+\boldsymbol{\beta}$ is another connection. In view of the above defining axioms, by (2.4) the $\mathcal{H}$-gauge transformation of a connection is $\boldsymbol{\omega}^{\gamma}:=\boldsymbol{\Psi}^{\star} \boldsymbol{\omega}=\boldsymbol{\gamma}^{-1} \boldsymbol{\omega} \boldsymbol{\gamma}+\boldsymbol{\gamma}^{-1} \boldsymbol{d} \boldsymbol{\gamma}$. At any point $\phi \in \Phi$, the horizontal complement to $V_{\phi} \Phi \subset T_{\phi} \Phi$ is $H_{\phi} \Phi:=\operatorname{ker} \boldsymbol{\omega}_{\mid \phi}$. We have then the horizontal subbundle s.t. $T \Phi=H \Phi \oplus V \Phi$. This allows to define the horizontal 
projection,

$$
\begin{aligned}
\left.\right|^{h}: \Gamma(T \Phi) & \rightarrow \Gamma(H \Phi), \\
\boldsymbol{X} & \mapsto \boldsymbol{X}^{h}:=\boldsymbol{X}-[\boldsymbol{\omega}(\boldsymbol{X})]^{v},
\end{aligned}
$$

as it is clear by (2.18) that $\boldsymbol{X}^{h} \in \operatorname{ker} \boldsymbol{\omega}$. One easily shows that, by $(2.17), R_{\gamma_{\star}} \boldsymbol{X}^{h}=$ $\left(R_{\gamma \star} \boldsymbol{X}\right)^{h}$, proving that the horizontal distribution thus defined is $\mathcal{H}$-equivariant, $R_{\gamma \star} H_{\phi} \mathcal{P}=$ $H_{\phi^{\gamma}} \Phi$, as it must.

The connection thus allows to define a notion of covariant derivation, $\boldsymbol{D}^{\omega}:=\left.\boldsymbol{d} \circ\right|^{h}$ : $\Omega_{\mathrm{eq}}^{\bullet}(\Phi, \boldsymbol{V}) \rightarrow \Omega_{\text {tens }}^{\bullet+1}(\Phi, \boldsymbol{V})$, that is $\boldsymbol{D}^{\omega} \boldsymbol{\alpha}\left(\boldsymbol{X}_{1}, \boldsymbol{X}_{2}, \ldots\right):=\boldsymbol{d} \boldsymbol{\alpha}\left(\boldsymbol{X}_{1}^{h}, \boldsymbol{X}_{2}^{h}, \ldots\right)$. The horizontality of the resulting form is obvious, and the preservation of the $\mathcal{H}$-equivariance is shown by $R_{\gamma}^{\star} \boldsymbol{D}^{\omega} \boldsymbol{\alpha}(\boldsymbol{X}, \ldots)=\boldsymbol{D}^{\omega} \boldsymbol{\alpha}\left(R_{\gamma_{\star}} \boldsymbol{X}, \ldots\right):=\boldsymbol{d} \boldsymbol{\alpha}\left(\left(R_{\gamma_{\star}} \boldsymbol{X}\right)^{h}, \ldots\right)=\boldsymbol{d} \boldsymbol{\alpha}\left(R_{\gamma_{\star}} \boldsymbol{X}^{h}, \ldots\right)=$ $\boldsymbol{d} R_{\gamma}^{\star} \boldsymbol{\alpha}\left(\boldsymbol{X}^{h}, \ldots\right)=\rho(\gamma)^{-1} \boldsymbol{d} \boldsymbol{\alpha}\left(\boldsymbol{X}^{h}, \ldots\right)=: \rho(\gamma)^{-1} \boldsymbol{D}^{\omega} \boldsymbol{\alpha}(\boldsymbol{X}, \ldots)$. On $\Omega_{\text {tens }}^{\bullet}(\Phi, \boldsymbol{V})$, the covariant derivative has the alternative expression $\boldsymbol{D}^{\boldsymbol{\omega}}=\boldsymbol{d}+\rho_{*}(\boldsymbol{\omega})$. And on $\Omega_{\text {basic }}^{\bullet}(\Phi, \boldsymbol{V})$, for which $\rho$ is trivial, it clearly reduces to the exterior derivative $\boldsymbol{D}^{\omega}=\boldsymbol{d}$. That is, $\boldsymbol{d}$ is a canonical covariant derivative on basic forms of $\Phi .^{8}$

The curvature of the connection is $\boldsymbol{\Omega}:=\boldsymbol{D}^{\boldsymbol{\omega}} \boldsymbol{\omega}=\boldsymbol{d} \boldsymbol{\omega}+\frac{1}{2}[\boldsymbol{\omega}, \boldsymbol{\omega}] \in \Omega_{\text {tens }}^{2}(\Phi$, Lie $\mathcal{H}),{ }^{9}$ and its $\mathcal{H}$-transformation is thus $\boldsymbol{\Omega}^{\gamma}=\gamma^{-1} \boldsymbol{\Omega} \gamma$. It is easily shown that on $\Omega_{\text {tens }}^{\bullet}(\Phi, \boldsymbol{V})$, $\boldsymbol{D}^{\boldsymbol{\omega}} \circ \boldsymbol{D}^{\boldsymbol{\omega}}=\rho_{*}(\boldsymbol{\Omega})$. The curvature satisfies the Bianchi identity $\boldsymbol{D}^{\boldsymbol{\omega}} \boldsymbol{\Omega}=\boldsymbol{d} \boldsymbol{\Omega}+[\boldsymbol{\omega}, \boldsymbol{\Omega}] \equiv 0$. As we have $\boldsymbol{\Omega}(\boldsymbol{X}, \boldsymbol{Y})=\boldsymbol{d} \boldsymbol{\omega}\left(\boldsymbol{X}^{h}, \boldsymbol{Y}^{h}\right)=-\boldsymbol{\omega}\left(\left[\boldsymbol{X}^{h}, \boldsymbol{Y}^{h}\right]\right)$, if $\left[\boldsymbol{X}^{h}, \boldsymbol{Y}^{h}\right]=[\boldsymbol{X}, \boldsymbol{Y}]^{h}$ then $\boldsymbol{\Omega} \equiv 0$. The curvature thus mesures the failure of (2.19) to be a Lie algebra morphism.

Connection associated with an equivariant metric. As verticality is canonically defined on $\Phi$, if it is endowed with a Riemannian metric $\boldsymbol{g}: \Gamma(T \Phi) \times \Gamma(T \Phi) \rightarrow \mathcal{C}^{\infty}(\Phi)$, the horizontal bundle $H \Phi$ can be defined as the $\boldsymbol{g}$-orthogonal complement of $V \Phi$. But for the horizontal distribution to be $\mathcal{H}$-equivariant, so must be the metric, $R_{\gamma}^{\star} \boldsymbol{g}=\boldsymbol{g}$ (the bundle structure of $\Phi$ must be respected). Thus, given a $\mathcal{H}$-equivariant metric $\boldsymbol{g}$ there is an associated connection $\boldsymbol{\omega}^{g}$ s.t. $\operatorname{ker} \boldsymbol{\omega}^{g}=\Gamma(V \Phi)^{\perp}:=\left\{\operatorname{ker} \boldsymbol{g}\left(\chi^{v},\right), \forall \chi^{v} \in \Gamma(V \Phi)\right\}$, and defined implicitly using (2.19) by $\boldsymbol{g}\left(\chi^{v}, \boldsymbol{X}-\left[\boldsymbol{\omega}^{g}(\boldsymbol{X})\right]^{v}\right)=0, \forall \chi \in$ Lie $\mathcal{H}$. Of course a metric is a priori no more canonical than a connection, and it is an even "richer" structure.

In the case of the $\mathcal{H}$-subbundle of connections (on $\mathcal{P}$ ) $\mathcal{A} \subset \Phi$, we have $T_{A} \mathcal{A} \simeq$ $\Omega_{\text {tens }}^{1}(\mathcal{P}, \operatorname{Lie} H)$. So, assuming the existence of a Hodge dual (thus of a metric) on $\mathcal{P}$, and for Lie $H$ semisimple, there is a 'natural' metric built via the Killing form on Lie $H$, i.e. the trace: $\stackrel{\circ}{\boldsymbol{g}}_{\mid A}: T_{A} \mathcal{A} \times T_{A} \mathcal{A} \rightarrow \mathbb{R},(\alpha, \beta) \mapsto \stackrel{\circ}{\boldsymbol{g}}_{\mid A}(\alpha, \beta):=\int \operatorname{Tr}(\alpha \wedge * \beta)$ (it is tacit that the arguments are compactly supported). The connection associated with this metric on $\mathcal{A}$ is introduced by Singer [39, 40], and termed the Singer-de Witt connection in $[7,8,10]$. Singer indeed defines the horizontal subspace as $H_{A} \mathcal{A}:=\left\{A+\alpha \mid D^{A \dagger} \alpha=0\right\}$ where $D^{A \dagger}:=(-)^{k} *^{-1} D^{A} *: \Omega_{\mathrm{eq}}^{k}(\mathcal{P}, \operatorname{Lie} H) \rightarrow \Omega_{\mathrm{tens}}^{k-1}(\mathcal{P}, \operatorname{Lie} H)$ it the adjoint of the covariant

\footnotetext{
${ }^{8}$ Which we knew already from the observation in section 2.1 that the basic complex $\left(\Omega_{\text {basic }}^{\bullet}(\Phi), \boldsymbol{d}\right)$ is a subcomplex of the de Rham complex of $\Phi$.

${ }^{9}$ It is the covariant derivative of the connection, but as the latter isn't tensorial, the second equality is not due to the alternative expression just mentioned with $\rho_{*}=$ ad, as the factor $\frac{1}{2}$ should tell. It is rather known as the Cartan structure equation for the curvature.
} 
derivative $D^{A}$ w.r.t. the inner product $\langle\zeta, \eta\rangle:=\int \operatorname{Tr}(\zeta \wedge * \eta)$, with $\zeta, \eta \in \Omega_{\mathrm{eq}}^{k}(\mathcal{P}$, Lie $H),{ }^{10}$ the orthogonality condition being $0=\stackrel{\circ}{\boldsymbol{g}}\left(D^{A} \chi, \alpha\right)=\int \operatorname{Tr}\left(D^{A} \chi \wedge * \alpha\right)=\int \operatorname{Tr}\left(\chi \wedge * D^{A \dagger} \alpha\right)$, $\forall \chi \in$ Lie $\mathcal{H}=\Omega_{\text {eq }}^{0}(\mathcal{P}, \operatorname{Lie} H)$ and the metric being non-degenerate.

Dual horizontalisation of forms. Since a connection allows to define the horizontal projection of vector fields, by duality it allows to define the "horizontalisation" of forms. Given $\boldsymbol{\alpha} \in \Omega^{\bullet}(\Phi)$, one defines the corresponding $\boldsymbol{\alpha}^{h}:=\left.\boldsymbol{\alpha} \circ\right|^{h} \in \Omega_{\text {hor }}(\Phi)$. In view of the expression for the horizontal projection (2.19), the explicit form of $\boldsymbol{\alpha}^{h}$ in terms of $\boldsymbol{\alpha}$ and $\boldsymbol{\omega}$ depends on the verticality property of $\boldsymbol{\alpha}$.

It is thus clear that if $\boldsymbol{\alpha} \in \Omega_{\mathrm{eq}}^{\bullet}(\Phi, \boldsymbol{V})$, then $\boldsymbol{\alpha}^{h} \in \Omega_{\text {tens }}^{\bullet}(\Phi, \boldsymbol{V})$. Indeed, much like the computation of the equivariance of $\boldsymbol{D}^{\omega} \boldsymbol{\alpha}$ above, we have $R_{\gamma}^{\star} \boldsymbol{\alpha}^{h}(\boldsymbol{X}, \ldots)=\boldsymbol{\alpha}^{h}\left(R_{\gamma \star} \boldsymbol{X}, \ldots\right):=\boldsymbol{\alpha}\left(\left(R_{\gamma \star} \boldsymbol{X}\right)^{h}, \ldots\right)=\boldsymbol{\alpha}\left(R_{\gamma \star} \boldsymbol{X}^{h}, \ldots\right)=R_{\gamma}^{\star} \boldsymbol{\alpha}\left(\boldsymbol{X}^{h}, \ldots\right)=$ $\rho(\gamma)^{-1} \boldsymbol{\alpha}\left(\boldsymbol{X}^{h}, \ldots\right)=: \rho(\gamma)^{-1} \boldsymbol{\alpha}^{h}(\boldsymbol{X}, \ldots)$. Idem, then, for twisted forms: for $\boldsymbol{\alpha} \in \Omega_{\mathrm{eq}}^{\bullet}(\Phi, C)$, we have $\boldsymbol{\alpha}^{h} \in \Omega_{\text {tens }}^{\bullet}(\Phi, C)$.

As illustrative applications, consider first the basis $\boldsymbol{d} \phi=\{\boldsymbol{d} A, \boldsymbol{d} \varphi\} \in \Omega_{\mathrm{eq}}^{1}(\Phi)$, to which is associated $\boldsymbol{d} \phi^{h}=\left\{\boldsymbol{d} A^{h}, \boldsymbol{d} \varphi^{h}\right\} \in \Omega_{\text {tens }}^{1}(\Phi)$, whose explicit form is, by (2.5) and (2.19),

$$
\boldsymbol{d} \phi_{\mid \phi}^{h}=\boldsymbol{d} \phi_{\mid \phi}-\delta_{\boldsymbol{\omega}} \phi \Rightarrow\left\{\begin{array}{l}
\boldsymbol{d} A_{\mid \phi}^{h}=\boldsymbol{d} A_{\mid \phi}-D^{A} \boldsymbol{\omega} \\
\boldsymbol{d} \varphi_{\mid \phi}^{h}=\boldsymbol{d} \varphi_{\mid \phi}+\rho_{*}(\boldsymbol{\omega}) \varphi
\end{array}\right.
$$

Remark that despite a superficial resemblance, these are not covariant derivative formulae ( $\phi$ is a point of $\Phi$ not an equivariant function on it). We find that applying the covariant derivative on $\boldsymbol{d} \phi^{h}$ results in $\boldsymbol{D}^{\omega} \boldsymbol{d} \phi^{h}=-\delta_{\boldsymbol{\Omega}} \phi$. Indeed,

$$
\begin{aligned}
\boldsymbol{D}^{\boldsymbol{\omega}} \boldsymbol{d} A^{h} & =\boldsymbol{d}\left(\boldsymbol{d} A^{h}\right)+\rho_{*}(\boldsymbol{\omega}) \boldsymbol{d} A^{h}=\boldsymbol{d}\left(-D^{A} \boldsymbol{\omega}\right)+\left[\boldsymbol{\omega}, \boldsymbol{d} A-D^{A} \boldsymbol{\omega}\right], \\
& =-D^{A}(\boldsymbol{d} \boldsymbol{\omega})-[\boldsymbol{d} A, \boldsymbol{\omega}]+[\boldsymbol{\omega}, \boldsymbol{d} A]-D^{A}\left(\frac{1}{2}[\boldsymbol{\omega}, \boldsymbol{\omega}]\right)=-D^{A} \boldsymbol{\Omega} . \\
\boldsymbol{D}^{\boldsymbol{\omega}} \boldsymbol{d} \varphi^{h} & =\boldsymbol{d}\left(\boldsymbol{d} \varphi^{h}\right)+\rho_{*}(\boldsymbol{\omega}) \boldsymbol{d} \varphi^{h}=\boldsymbol{d}\left(\rho_{*}(\boldsymbol{\omega}) \varphi\right)+\rho_{*}(\boldsymbol{\omega})\left(\boldsymbol{d} \varphi+\rho_{*}(\boldsymbol{\omega}) \varphi\right), \\
& =\rho_{*}(\boldsymbol{d} \boldsymbol{\omega}) \varphi+\rho_{*}(\boldsymbol{\omega} \wedge \boldsymbol{\omega}) \varphi=\rho_{*}(\boldsymbol{\Omega}) \varphi .
\end{aligned}
$$

Equation (2.20) gives, for example, a general expression for 1-forms: given $\boldsymbol{\alpha}=\alpha(\boldsymbol{d} \phi ; \phi) \in$ $\Omega^{1}(\Phi)$, we have

$$
\boldsymbol{\alpha}^{h}=\alpha\left(\boldsymbol{d} \phi^{h} ; \phi\right)=\boldsymbol{\alpha}-\iota_{[\boldsymbol{\omega}]^{v}} \boldsymbol{\alpha}=\boldsymbol{\alpha}-\alpha\left(\delta_{\boldsymbol{\omega}} \phi ; \phi\right) \quad \in \Omega_{\mathrm{hor}}^{1}(\Phi)
$$

Consider then $\boldsymbol{d} F, \boldsymbol{d} D^{A} \varphi \in \Omega_{\mathrm{eq}}^{1}(\Phi)$ to which, by (2.10), (2.14) and (2.19), one associates $\boldsymbol{d} F^{h},\left(\boldsymbol{d} D^{A} \varphi\right)^{h} \in \Omega_{\text {tens }}^{1}(\Phi)$ given respectively by

$$
\boldsymbol{d} F^{h}=\boldsymbol{d} F-[F, \boldsymbol{\omega}] \quad \text { and } \quad\left(\boldsymbol{d} D^{A} \varphi\right)^{h}=\boldsymbol{d} D^{A} \varphi+\rho_{*}(\boldsymbol{\omega}) D^{A} \varphi
$$

\footnotetext{
${ }^{10}$ This generalises the construction of the codifferential $\delta:=(-)^{k} *^{-1} d *: \Omega^{k}(\mathcal{P}, \mathbb{R}) \rightarrow \Omega^{k-1}(\mathcal{P}, \mathbb{R})$ as the adjoint of the exterior derivative $d$ w.r.t. the inner product $\langle\zeta, \eta\rangle:=\int \zeta \wedge * \eta$ for $\zeta, \eta \in \Omega^{k}(\mathcal{P}, \mathbb{R})$.
} 
But remark that since $F, D^{A} \varphi \in \Omega_{\text {eq }}^{0}(\Phi)=\Omega_{\text {tens }}^{0}(\Phi)$ we have $D^{\omega} F, D^{\omega}\left(D^{A} \varphi\right) \in \Omega_{\text {tens }}^{1}(\Phi)$ with explicit expressions,

$$
\boldsymbol{D}^{\boldsymbol{\omega}} F=\boldsymbol{d} F+[\boldsymbol{\omega}, F] \quad \text { and } \quad \boldsymbol{D}^{\boldsymbol{\omega}}\left(D^{A} \varphi\right)=\boldsymbol{d} D^{A} \varphi+\rho_{*}(\boldsymbol{\omega}) D^{A} \varphi
$$

In this occasion then, (2.24) are indeed covariant derivative expressions. Which is not surprising as, by definition, $\boldsymbol{D}^{\omega}:=\left.\boldsymbol{d} \circ\right|^{h}$ on $\Omega_{\mathrm{eq}}^{\bullet}(\Phi)$, so that the 'horizontalisation' of $\boldsymbol{d} F$ and $\boldsymbol{d} D^{A} \varphi$ is precisely the definition of the covariant derivative of $F$ and $D^{A} \varphi$. It is then immediate that,

$$
\boldsymbol{D}^{\boldsymbol{\omega}}\left(\boldsymbol{d} F^{h}\right)=\boldsymbol{D}^{\boldsymbol{\omega}} \circ \boldsymbol{D}^{\boldsymbol{\omega}} F=[\boldsymbol{\Omega}, F] \quad \text { and } \quad \boldsymbol{D}^{\boldsymbol{\omega}}\left(\boldsymbol{d} D^{A} \varphi\right)^{h}=\boldsymbol{D}^{\boldsymbol{\omega}} \circ \boldsymbol{D}^{\boldsymbol{\omega}}\left(D^{A} \varphi\right)=\rho_{*}(\boldsymbol{\Omega}) D^{A} \varphi
$$

A case of special relevance to our general purpose is that to $\boldsymbol{\alpha} \in \Omega_{\text {inv }}^{\bullet}(\Phi)$ one then associates $\boldsymbol{\alpha}^{h} \in \Omega_{\text {basic }}^{\bullet}(\Phi)$. In this case only, one may observe that both $\boldsymbol{D}^{\boldsymbol{\omega}} \boldsymbol{\alpha}:=(\boldsymbol{d} \boldsymbol{\alpha})^{h}$ and $\boldsymbol{d} \boldsymbol{\alpha}^{h}$ are basic forms, yet in general $\boldsymbol{d}^{h} \neq \boldsymbol{D}^{\omega} \boldsymbol{\alpha}$. For $\boldsymbol{\alpha}=\alpha(\boldsymbol{d} \phi ; \phi) \in \Omega_{\text {inv }}^{1}(\Phi)$ in particular, we prove in appendix A that the following formula holds,

$$
\boldsymbol{D}^{\boldsymbol{\omega}} \boldsymbol{\alpha}=\boldsymbol{d} \boldsymbol{\alpha}^{h}+\iota_{[\boldsymbol{\Omega}]^{v}} \boldsymbol{\alpha}=\boldsymbol{d} \boldsymbol{\alpha}^{h}+\alpha\left(\delta_{\boldsymbol{\Omega}} \phi ; \phi\right),
$$

using the notation introduced in equation (2.5). So that, only in the special case of a flat $\stackrel{\circ}{\omega}$ do we get $\boldsymbol{D}^{\stackrel{\leftrightarrow}{\alpha}}=\boldsymbol{d} \boldsymbol{\alpha}^{h}$.

Ambiguity in the choice of connection. As already seen, connections are noncanonical and form an affine space. So, the above 'horizontalisation' and 'basification' of forms suffers from an ambiguity due to an a priori arbitrariness in the choice of connection. Given any $\boldsymbol{\beta} \in \Omega_{\text {tens }}^{1}(\Phi$, lie $\mathcal{H})$, both $\boldsymbol{\omega}$ and $\boldsymbol{\omega}^{\prime}=\boldsymbol{\omega}+\boldsymbol{\beta}$ are valid choices of connections. Given $\boldsymbol{\alpha} \in \Omega^{\bullet}(\Phi)$, denote $\boldsymbol{\alpha}_{\boldsymbol{\omega}}^{h}$ and $\boldsymbol{\alpha}_{\boldsymbol{\omega}^{\prime}}^{h}$ the corresponding horizontal forms obtain by the above procedure respectively through $\boldsymbol{\omega}$ and $\boldsymbol{\omega}^{\prime}$. One may ask how they are related. The answer is easily found for 1-forms via a well known trick (familiar e.g. from the proof of the Chern-Weil homomorphism theorem).

Consider the affine curve in the space of connections $\boldsymbol{\omega}_{\tau}:=\boldsymbol{\omega}+\tau \boldsymbol{\beta}$, with $\tau \in[0,1]$, s.t. $\boldsymbol{\omega}_{0}=\boldsymbol{\omega}$ and $\boldsymbol{\omega}_{1}=\boldsymbol{\omega}^{\prime}$. Given $\boldsymbol{\alpha} \in \Omega^{1}(\Phi)$, we have by definition $\boldsymbol{\alpha}_{\boldsymbol{\omega}_{\tau}}^{h}=\boldsymbol{\alpha}-\iota_{\left[\boldsymbol{\omega}_{\tau}\right]^{v}} \boldsymbol{\alpha} \in \Omega_{\mathrm{hor}}^{1}(\Phi)$. We have then the generic result,

$$
\begin{aligned}
\int_{0}^{1} d \tau \frac{d}{d \tau} \boldsymbol{\alpha}_{\omega_{\tau}}^{h} & =\boldsymbol{\alpha}_{\omega^{\prime}}^{h}-\boldsymbol{\alpha}_{\omega}^{h}=-\iota_{[\beta]^{v}} \boldsymbol{\alpha}, \\
& \hookrightarrow \quad \boldsymbol{\alpha}_{\omega^{\prime}}^{h}=\boldsymbol{\alpha}_{\omega}^{h}-\iota_{[\beta]^{v}} \boldsymbol{\alpha} \quad \in \Omega_{\mathrm{hor}}^{1}(\Phi) .
\end{aligned}
$$

In particular, considering $\boldsymbol{d} \phi \in \Omega_{\mathrm{eq}}^{1}(\Phi)$ we have

$$
\boldsymbol{d} \phi_{\boldsymbol{\omega}^{\prime}}^{h}=\boldsymbol{d} \phi_{\boldsymbol{\omega}}^{h}-\iota_{[\boldsymbol{\beta}]^{v}} \boldsymbol{d} \phi=\boldsymbol{d} \phi_{\boldsymbol{\omega}}^{h}-\delta_{\boldsymbol{\beta}} \phi \quad \in \Omega_{\mathrm{tens}}^{1}(\Phi) .
$$

From this, one can in principle work out the precise relation between $\boldsymbol{\alpha}_{\boldsymbol{\omega}^{\prime}}^{h}=\alpha\left(\wedge^{\bullet} \boldsymbol{d} \phi_{\boldsymbol{\omega}^{\prime}}^{h} ; \phi\right)$ and $\boldsymbol{\alpha}_{\omega}^{h}=\alpha\left(\wedge^{\bullet} \boldsymbol{d} \phi_{\omega}^{h} ; \phi\right)$. We do not do so as we will not make use of such a general result. 
In the case of special interest to us $\boldsymbol{\alpha}=\alpha(\boldsymbol{d} \phi ; \phi) \in \Omega_{\mathrm{inv}}^{1}(\Phi)$, as the above procedure produces basic forms, we alter the notation in eq. (2.28) to have more suggestively,

$$
\begin{aligned}
\boldsymbol{\alpha}_{\boldsymbol{\omega}^{\prime}}^{b} & =\boldsymbol{\alpha}_{\boldsymbol{\omega}}^{b}-\iota_{[\boldsymbol{\beta}]^{v}} \boldsymbol{\alpha} \quad \in \Omega_{\text {basic }}^{1}(\Phi), \\
& =\boldsymbol{\alpha}_{\boldsymbol{\omega}}^{b}-\alpha\left(\delta_{\boldsymbol{\beta}} \phi ; \phi\right) .
\end{aligned}
$$

Consequently, the covariant derivatives are related by

$$
\boldsymbol{d}_{\boldsymbol{\omega}^{\prime}}^{b}=\boldsymbol{d} \boldsymbol{\alpha}_{\boldsymbol{\omega}}^{b}-\boldsymbol{d} \alpha\left(\delta_{\boldsymbol{\beta}} \phi ; \phi\right) \quad \in \Omega_{\mathrm{basic}}^{2}(\Phi) .
$$

These two results we will use when considering the basic presymplectic structure of gauge theories.

The ambiguity in the procedure being worked out, it may still be that there is a choice of connection more natural than others. Such would be the case, as seen above, if it is associated with a natural bundle metric.

\subsubsection{Variational twisted connections}

We briefly review a notion of connection which, relatively to the aim of covariant differentiation, is a slight generalisation of Ehresmann's. A variational twisted connection is by definition $\tilde{\boldsymbol{\omega}} \in \Omega_{\text {eq }}^{1}(\Phi, \operatorname{Lie} G)^{C}$ satisfying:

$$
\begin{aligned}
& R_{\gamma}^{\star} \tilde{\boldsymbol{\omega}}_{\mid \phi^{\gamma}}=C(\phi, \gamma)^{-1} \tilde{\boldsymbol{\omega}}_{\mid \phi} C(\phi, \gamma)+C(\phi, \gamma)^{-1} \boldsymbol{d} C(, \gamma)_{\mid \phi}, \\
& \tilde{\boldsymbol{\omega}}_{\mid \phi}\left(\chi_{\phi}^{v}\right)=\left.\frac{d}{d \tau} C(\phi, \exp (\tau \chi))\right|_{\tau=0}, \quad \chi \in \operatorname{Lie} \mathcal{H} .
\end{aligned}
$$

It is non-canonical, and from the above defining axioms follows that the space of twisted connections is affine and modelled on the vector space $\Omega_{\text {tens }}^{1}(\Phi, \operatorname{Lie} G)^{C}$ : for $\boldsymbol{\beta} \in \Omega_{\text {tens }}^{1}(\Phi, \operatorname{Lie} G)^{C}$, we have that $\tilde{\boldsymbol{\omega}}^{\prime}=\tilde{\boldsymbol{\omega}}+\boldsymbol{\beta}$ is another twisted connection. In view of (2.32)-(2.33), and by (2.4), it takes some doing to show that the $\mathcal{H}$-gauge transformation of a twisted connection is $\boldsymbol{\omega}^{\gamma}:=\boldsymbol{\Psi}^{\star} \boldsymbol{\omega}=C(\boldsymbol{\gamma})^{-1} \boldsymbol{\omega} C(\boldsymbol{\gamma})+C(\boldsymbol{\gamma})^{-1} \boldsymbol{d} C(\boldsymbol{\gamma})$, where we introduce the convenient notation $C(\gamma(\phi)):=C(\phi, \gamma(\phi))$.

A covariant derivation is defined on twisted forms by $\boldsymbol{D}^{\tilde{\boldsymbol{\omega}}}:=\boldsymbol{d}+\tilde{\boldsymbol{\omega}}: \Omega_{\text {tens }}^{\bullet}(\Phi, \boldsymbol{V})^{C} \rightarrow$ $\Omega_{\text {tens }}^{\bullet+1}(\Phi, \boldsymbol{V})^{C}$, and of course $\boldsymbol{D}^{\tilde{\boldsymbol{\omega}}}=\boldsymbol{d}$ on $\Omega_{\text {basic }}^{\bullet}(\Phi, \boldsymbol{V})$. The curvature of a twisted connection is defined via Cartan's structure equation, $\tilde{\boldsymbol{\Omega}}:=\boldsymbol{d} \tilde{\boldsymbol{\omega}}+\frac{1}{2}[\tilde{\boldsymbol{\omega}}, \tilde{\boldsymbol{\omega}}] \in \Omega_{\text {tens }}^{2}(\Phi, \operatorname{Lie} G)^{C}$, thus it satisfies a Bianchi identity $\boldsymbol{D}^{\tilde{\omega}} \tilde{\boldsymbol{\Omega}} \equiv 0$, and has $\mathcal{H}$-transformation $\tilde{\boldsymbol{\Omega}}^{\boldsymbol{\gamma}}=C(\boldsymbol{\gamma})^{-1} \tilde{\boldsymbol{\Omega}} C(\boldsymbol{\gamma})$. As usual $\boldsymbol{D}^{\tilde{\omega}} \circ \boldsymbol{D}^{\tilde{\omega}}=\tilde{\Omega}$.

Twisted connection are the adequate tool to tackle general non-invariant gauge theories (case 3, just above 2.2). As we have seen, the Lagrangian of such a theory has $\mathcal{H}$-equivariance $R_{\gamma}^{\star} L=L+c(, \gamma)$, whose infinitesimal version gives the classical gauge anomaly $\boldsymbol{L}_{\chi^{v}} L=\left.\frac{d}{d \tau} c(, \exp \tau \chi)\right|_{\tau=0}=: \alpha(\chi$,$) . Considering the associated functional Z:=$ $\exp i \int L \in \Omega_{\text {tens }}^{0}(\Phi, \mathbb{C})^{C}$, s.t. $R_{\gamma}^{\star} Z=C(, \gamma)^{-1} Z$ with $C(, \gamma):=\exp -i \int c(, \gamma) \in \mathrm{U}(1)$, we see that a non-invariant theory defines a section of the twisted associated line bundle $\tilde{\boldsymbol{E}}:=\Phi \times_{C} \mathbb{C}$. The adapted abelian twisted connection is $\tilde{\boldsymbol{\omega}} \in \Omega_{\mathrm{eq}}^{1}(\Phi, i \mathbb{R})^{C}$ s.t.

$$
\begin{aligned}
R_{\gamma}^{\star} \tilde{\boldsymbol{\omega}}_{\mid \phi^{\gamma}} & =\tilde{\boldsymbol{\omega}}_{\mid \phi}-i \int \boldsymbol{d} c(, \gamma)_{\mid \phi}, \\
\tilde{\boldsymbol{\omega}}_{\mid \phi}\left(\chi_{\phi}^{v}\right) & =-i \int \alpha(\chi, \phi)=:-i a(\chi, \phi) .
\end{aligned}
$$


The verticality property of the twisted connection encodes the gauge anomaly, and consequently the tensoriality of the curvature $\tilde{\boldsymbol{\Omega}}=\boldsymbol{d} \tilde{\boldsymbol{\omega}}$ encodes the Wess-Zumino consistency condition:

$$
\begin{array}{r}
\tilde{\boldsymbol{\Omega}}\left(\chi^{v}, \eta^{v}\right)=\boldsymbol{d} \tilde{\boldsymbol{\omega}}\left(\chi^{v}, \eta^{v}\right)=\chi^{v} \cdot \tilde{\boldsymbol{\omega}}\left(\eta^{v}\right)-\eta^{v} \cdot \tilde{\boldsymbol{\omega}}\left(\chi^{v}\right)-\tilde{\boldsymbol{\omega}}\left(\left[\chi^{v}, \eta^{v}\right]\right) \equiv 0 \\
\hookrightarrow \chi^{v} \cdot a(\eta, \phi)-\eta^{v} \cdot a(\chi, \phi)-a([\chi, \eta], \phi)=0 .
\end{array}
$$

To be compared e.g. to eq. (8.62) and eq. (10.76) in [48], or eq. (12.25) in [49].

Interestingly, one find a 'modified basic action' from the twisted covariant derivative of $Z$. Indeed,

$$
\boldsymbol{D}^{\tilde{\boldsymbol{\omega}}} Z=\boldsymbol{d} Z+\tilde{\boldsymbol{\omega}} Z=\left(i \boldsymbol{d} \int L+\tilde{\boldsymbol{\omega}}\right) Z=(i \boldsymbol{d} S+\tilde{\boldsymbol{\omega}}) Z
$$

where $S:=\int L$ is the classical action. Since $Z$ and $D^{\tilde{\omega}} Z \in \Omega_{\text {tens }}^{\bullet}(\Phi, \mathbb{C})^{C}$, we have that $i \boldsymbol{d} S+\tilde{\boldsymbol{\omega}} \in \Omega_{\text {basic }}^{1}(\Phi, i \mathbb{R})$. This is easily checked explicitly by $(2.34)-(2.35)$,

$$
\begin{gathered}
R_{\gamma}^{\star}(i \boldsymbol{d} S+\tilde{\boldsymbol{\omega}})=i \boldsymbol{d}\left(\int L+c(, \gamma)\right)+\tilde{\boldsymbol{\omega}}-i \int \boldsymbol{d} c(, \gamma)=i \boldsymbol{d} S+\tilde{\boldsymbol{\omega}}, \\
(i \boldsymbol{d} S+\tilde{\boldsymbol{\omega}})\left(\chi^{v}\right)=i \int \boldsymbol{L}_{\chi^{v}} L+\tilde{\boldsymbol{\omega}}\left(\chi^{v}\right)=i \int \alpha(\chi,)-i \int \alpha(\chi,)=0 .
\end{gathered}
$$

The quantity $\boldsymbol{d} S-i \tilde{\boldsymbol{\omega}}$ is a generalisation of the notion of Wess-Zumino 'improved' action [50, 51]. Indeed, if the connection is locally exact, $\tilde{\boldsymbol{\omega}}=\boldsymbol{d} \tilde{\boldsymbol{\varpi}}$, then $S-i \tilde{\boldsymbol{\omega}} \in \Omega_{\text {basic }}^{0}(\Phi, \mathbb{R})=$ $\Omega_{\mathrm{inv}}^{0}(\Phi, \mathbb{R})$, and $\tilde{\varpi}$ is a Wess-Zumino functional. In section 2.3.1 we will see how such a term can be built explicitly from the cocycle $c$ and a dressing field.

What is done here for classical gauge anomalies holds also for quantum gauge anomalies, see sections of 2.3 and 4.2 of [33], and [47] for a full exposition of the geometry of twisted connections

The extraction of a basic action 0-form above is quite incidental. As a twisted connection is a priori not meant to split the SES (2.2), it does not provide a notion of horizontality on $\Phi$ and thus would not be used to extract the horizontal part of any given form. A fortiori, it is unlikely to provide a general strategy to build basic forms. In the following section we discuss a general method designed to do just that.

\subsection{The dressing field method}

The dressing field method (DFM) is a systematic way to build basic forms on a bundle, thus to obtain gauge-invariants in gauge theory. It has gradually developed [52-54] in recent years, and its immediate implications regarding the philosophy of gauge theories relevant to the present paper - has been first expound in [55]. In relation to the topic of the presymplectic structure of gauge theories, the DFM is the geometric underpinning of the so-called edge modes [5, 14-17, 23-25]. A relatively complete and self-contained exposition can be found in sections 3 and 4.3 of [33]. But in the interest of the reader, we give here a summary of the essentials of the method.

Consider a $H$-principal bundle $\mathcal{P}$ with connection $A$, and $\alpha \in \Omega_{\text {tens }}^{\bullet}(\mathcal{P}, V)$. Let $K \triangleleft H$ be a normal subgroup of the structure group, so that the quotient $H / K=J$ is a group. Correspondingly we have the gauge subgroups $\mathcal{K}, \mathcal{J} \subset \mathcal{H}$. Also, let $G$ be a group s.t. $G \subset H$. 
We define the space of $K$-dressing fields as $\mathcal{D} r[K, G]:=\left\{u: \mathcal{P} \rightarrow G \mid R_{k}^{*} u=k^{-1} u, \forall k \in\right.$ $K$ \}, a definition that implies that the $\mathcal{K}$-gauge transformation on $\mathcal{P}$ of a dressing is $u^{\gamma}=$ $\gamma^{-1} u$. By means of the dressing field, one defines the map $f_{u}: \mathcal{P} \rightarrow \mathcal{P} / K=\mathcal{P}^{\prime}, p \mapsto$ $f_{u}(p):=p u(p)$, where $\mathcal{P}^{\prime}$ is a $J$-principal subbundle of $\mathcal{P}$. In other words, the existence of a $K$-dressing field implies that the bundle $\mathcal{P}$ is trivial along $K: \mathcal{P} \simeq \mathcal{P}^{\prime} \times K$. This map satisfies $f_{u} \circ R_{k}=f_{u}$, so $R_{k}^{*} \circ f_{u}^{*}=f_{u}^{*}$ and $f_{u *} X^{v}=0$ for $X \in \operatorname{Lie} K$ (it kills vertical vector fields generated by the action of $K$ ). Therefore, the following dressed fields

$$
A^{u}:=f_{u}^{*} A=u^{-1} A u+u^{-1} d u, \quad \text { and } \quad \alpha^{u}:=f_{u}^{*} \alpha=\rho(u)^{-1} \alpha,
$$

are $K$-basic on $\mathcal{P}$, thus descend on $\mathcal{P}^{\prime}$. This implies that both are $\mathcal{K}$-invariant: $\left(A^{u}\right)^{\gamma}=A^{u}$ and $\left(\alpha^{u}\right)^{\gamma}=\alpha^{u}$ for $\gamma \in \mathcal{K}$. In particular, the curvature $F \in \Omega_{\text {tens }}^{2}(\mathcal{P}, \operatorname{Lie} H)$ and $\varphi \in$ $\Omega_{\text {tens }}^{0}(\mathcal{P}, V) \simeq \Gamma(E)$ dress as $F^{u}=u^{-1} F u=d A^{u}+\frac{1}{2}\left[A^{u}, A^{u}\right]$ (which is then the curvature of $\left.A^{u}\right)$ and $\varphi^{u}=\rho(u)^{-1} \varphi$.

Let us emphasize an important fact: it is clear from the definition that $u \notin \mathcal{K}$, so that despite the formal resemblance (2.40) are not gauge transformations. This means, in particular, that the dressed connection is not a $H$-connection, $A^{u} \notin \mathcal{A}$, and a fortiori is not a point in the gauge $\mathcal{K}$-orbit $\mathcal{O}_{\mathcal{K}}[A]$ of $A$, so that $A^{u}$ must not be confused with a gauge-fixing of $A$.

We also point out that, with minimal adjustments, the above results can be extended to the case $G \supset H$ : one needs only to assume that $G$ is (a subgroup of) the structure group of a bigger principal bundle of which $\mathcal{P}$ is a subbundle/a reduction. This is typically the case for Cartan geometries, on which gauge gravity theories are based. See section 2.2, and footnote 12 , in [33].

Finally, remark that if $K=H$ the bundle is trivial, $\mathcal{P} \simeq M \times H$, and $A^{u}, \alpha^{u} \in$ $\Omega_{\text {basic }}^{\bullet}(\mathcal{P})$. This means in particular that in this case there is a 1:1 association between the dressed fields $\phi^{u}=\left(A^{u}, \varphi^{u}\right)$ and the $\mathcal{H}$-gauge orbit $\mathcal{O}_{\mathcal{H}}[\phi]$. So, $\phi^{u}$ can be thought of as a 'coordinatisation' for the gauge class $[\phi]$ such that $\Phi^{u} \simeq \Phi / \mathcal{H}=\mathcal{M}$. This must be qualified, as the dressed fields may exhibit residual transformations.

Residual gauge symmetry. If $K \triangleleft H$, one expects (2.40) to display a residual $\mathcal{J}$ gauge transformations which will depend on the $\mathcal{J}$-transformation of $u$ (that of $A$, $\alpha$ being known already), the latter in turn given by its $J$-equivariance. We will not linger on the details here, referring to section 3.2 of [33] for details, only to mention one interesting case which is when the $K$-dressing field has $J$-equivariance $R_{j}^{*} u=j^{-1} u j$ so that its $\mathcal{J}$-gauge transformation is $u^{\eta}=\eta^{-1} u \eta$ and that of the dressed fields is then,

$$
\left(A^{u}\right)^{\eta}:=\Psi^{*} A^{u}=\eta^{-1} A^{u} \eta+\eta^{-1} d \eta, \quad \text { and } \quad\left(\alpha^{u}\right)^{\eta}:=\Psi^{*} \alpha^{u}=\rho(\eta)^{-1} \alpha^{u},
$$

for $\Psi \in \operatorname{Aut}_{v}(\mathcal{P}, J) \simeq \operatorname{Aut}_{v}\left(\mathcal{P}^{\prime}\right)(J$-automorphism $)$ s.t. $\Psi(p)=p \eta(p)$ with $\eta \in \mathcal{J}$. This can be checked algebraically from $\left(A^{u}\right)^{\eta}=\left(A^{\eta}\right)^{u^{\eta}}$.

In the rest of this paper we will consider the case of dressing fields $u \in \operatorname{Dr}[H, G]$ for simplicity. ${ }^{11}$ Yet, even in this case the dressed fields may display another form of residual

\footnotetext{
${ }^{11}$ Although, as we have remarked, it is a strong constraint on the topology of $\mathcal{P}$ - it is trivial — which in turn has interpretive implications [55] quite significant for the 'edge mode' strategy, as argued in [33] and as we will further comment ahead.
} 
transformations worth stressing, stemming from an ambiguity in the choice of dressing field.

Ambiguity in the choice of a dressing field. Given their defining property, two dressing fields $u, u^{\prime} \in \mathcal{D} r[H, G]$ may a priori be related by $u^{\prime}=u \xi$, with $\xi \in \mathcal{G}:=$ $\left\{\xi: \mathcal{P} \rightarrow G \mid R_{h}^{*} \xi=\xi\right\}$. By analogy with the notation for the action of the gauge group $\mathcal{H}$, let us denote the action of $\mathcal{G}$ on $\operatorname{Dr}[H, G]$ as $u^{\xi}=u \xi$. By definition $\mathcal{G}$ acts trivially on $A$ and $\alpha$, a fact we denote by $A^{\xi}=A$ and $\alpha^{\xi}=\alpha$. On the other hand, it is clear how $\mathcal{G}$ must act on dressed fields:

$$
\left(A^{u}\right)^{\xi}:=A^{u^{\xi}}=A^{u \xi}=\xi^{-1} A^{u} \xi+\xi^{-1} d \xi, \quad \text { and } \quad\left(\alpha^{u}\right)^{\xi}:=\alpha^{u^{\xi}}=\alpha^{u \xi}=\rho(\xi)^{-1} \alpha^{u},
$$

which implies in particular $\left(F^{u}\right)^{\xi}=\xi^{-1} F^{u} \xi$ and $\left(\varphi^{u}\right)^{\xi}=\rho(\xi)^{-1} \varphi^{u}$.

This invites to think of the space of dressed fields $\Phi^{u}$ as fibered by the right action of $\mathcal{G}$, noted then $R_{\xi} \phi^{u}:=\left(\phi^{u}\right)^{\xi}$, so that $\Phi^{u}$ is a $\mathcal{G}$-principal bundle over $\Phi^{u} / \mathcal{G}:=\mathcal{M}^{u}$. As such, and in complete analogy with $\Phi$, it gives rise to a SES

$$
0 \longrightarrow \operatorname{Aut}_{v}\left(\Phi^{u}\right) \simeq \mathcal{G} \stackrel{\iota}{\longrightarrow} \operatorname{Aut}\left(\Phi^{u}\right) \stackrel{\tilde{\pi}}{\longrightarrow} \operatorname{Diff}\left(\mathcal{M}^{u}\right) \longrightarrow 0,
$$

where $\operatorname{Aut}\left(\Phi^{u}\right)$ is the automorphism group defined as usual, and the subgroup of vertical automorphisms is isomorphic to the gauge group $\mathcal{G}:=\left\{\boldsymbol{\xi}: \Phi^{u} \rightarrow \mathcal{G} \mid R_{\xi}^{\star} \boldsymbol{\xi}=\xi^{-1} \boldsymbol{\xi} \xi\right\}$. Its infinitesimal version is,

$$
0 \longrightarrow \Gamma_{\mathcal{G}}\left(V \Phi^{u}\right) \simeq \operatorname{Lie} \mathcal{G} \stackrel{\iota}{\longrightarrow} \Gamma_{\mathcal{G}}\left(T \Phi^{u}\right) \stackrel{\pi_{\star}}{\longrightarrow} \Gamma\left(T \mathcal{M}^{u}\right) \longrightarrow 0,
$$

As usual, the action by pullback of $\operatorname{Aut}_{v}\left(\Phi^{u}\right)$ on elements of $\Omega^{\bullet}\left(\Phi^{u}\right)$ defines their $\mathcal{G}$-gauge transformations which is thus determined by their $\mathcal{G}$-equivariance and verticality properties, i.e. the result of their evaluation on $\zeta^{v} \in \Gamma\left(V \Phi^{u}\right)$ for $\zeta \in$ Lie $\mathcal{G}$. We will have more to say on this shortly.

Or course, $\left(\phi^{u}\right)^{\xi}$ is $H$-basic (and $\mathcal{H}$-invariant) $\forall \xi \in \mathcal{G}$, so any given representative in the $\mathcal{G}$-orbit $\mathcal{O}_{\mathcal{G}}\left[\phi^{u}\right]$ is as good a coordinatisation for $[\phi] \in \mathcal{M}$ as any other. Said otherwise there is a $1: 1$ correspondence $\mathcal{O}_{\mathcal{H}}[\phi] \sim \mathcal{O}_{\mathcal{G}}\left[\phi^{u}\right]$. Which means that, contrary to a first analysis, it is $\Phi^{u} / \mathcal{G}:=\mathcal{M}^{u}$ that is isomorphic to $\Phi / \mathcal{H}=\mathcal{M}$ (not $\Phi^{u}$ ). As the latter is the physical state space, it follows that $\mathcal{G}$ is not a transformation group of physical states (it acts trivially on them). Rather, as we know, $\operatorname{Diff}(\Phi / \mathcal{H}) \simeq \operatorname{Diff}\left(\Phi^{u} / \mathcal{G}\right)$ is, with infinitesimal counterpart $\Gamma(\Phi / \mathcal{H}) \simeq \Gamma\left(\Phi^{u} / \mathcal{G}\right)$.

This being clarified, there are only two relevant options regarding the physical status of the group $\mathcal{G}$ : either it is an interesting new gauge symmetry, as is the case in gauge gravity theories where $\mathcal{G}=\mathcal{G} \mathcal{L}(n)$ is the group of local coordinate changes (see, section 5.3.1.b in [33]), and as such the associated Noether charges may be observables when 'measured' against background field configurations for whom elements of $\mathcal{G}$ are Killing symmetries (a topic we address first for $\mathcal{H}$ in section 3.2, then for $\mathcal{G}$ in section 4.2). Or there are compelling reasons as to why the group $\mathcal{G}$ must be 'small' compared to $\mathcal{H}$ (perhaps even reduced to a global/rigid or discrete group). For either options to stand a chance of being realised, a dressing field must be introduced not by hand as new degrees of freedom, but built from elements of the initial field space.

This suggests to consider field-dependent dressing fields which, as it turns out, also permit to build basic forms on the $\mathcal{H}$-bundle $\Phi$. 
Field-dependent dressing fields. A $\Phi$-dependent dressing field is a map $\boldsymbol{u}: \Phi \rightarrow$ $\mathcal{D} r[H, G], \phi \mapsto \boldsymbol{u}(\phi)$, thus satisfying $R_{\gamma}^{\star} \boldsymbol{u}=\gamma^{-1} \boldsymbol{u}$ - i.e. $\boldsymbol{u}\left(\phi^{\gamma}\right)=\gamma^{-1} \boldsymbol{u}(\phi)-$ for $\gamma \in \mathcal{H}{ }^{12}$ Given the above considerations, it allows to define

$$
\begin{aligned}
\mathrm{F}_{\boldsymbol{u}}: \Phi & \rightarrow \mathcal{M} \\
\phi & \mapsto \mathrm{F}_{\boldsymbol{u}}(\phi):=\phi^{\boldsymbol{u}}=\left(A^{\boldsymbol{u}}, \varphi^{\boldsymbol{u}}\right), \quad \text { s.t. } \quad \mathrm{F}_{\boldsymbol{u}} \circ R_{\gamma}=\mathrm{F}_{\boldsymbol{u}} .
\end{aligned}
$$

This map in a sense realises the projection map $\pi$ of $\Phi$. So clearly, $\Gamma(V \Phi) \subset \operatorname{ker} \mathrm{F}_{\boldsymbol{u}}$ : for $\chi^{v} \in$ $\Gamma(V \Phi)$ generated by $\chi \in$ Lie $\mathcal{H}$, we have $\mathrm{F}_{\boldsymbol{u} \star} \chi_{\phi}^{v}=\left.\frac{d}{d \tau}\left(\mathrm{F}_{\boldsymbol{u}} \circ R_{e^{\tau} \chi}\right)(\phi)\right|_{\tau=0}=\left.\frac{d}{d \tau} \mathrm{F}_{\boldsymbol{u}}(\phi)\right|_{\tau=0}=0$. Despite the formal resemblance with a vertical automorphism, $\mathrm{F}_{\boldsymbol{u}} \notin \operatorname{Aut}_{v}(\Phi)$, as is clear from the fact that $\boldsymbol{u} \notin \mathcal{H}$. Yet, in exact analogy with the computation of $\boldsymbol{\Psi}_{\star} \boldsymbol{X}$ for $\boldsymbol{X} \in \Gamma(T \Phi)$, eq. (2.4), (which owes nothing to the $\mathcal{H}$-equivariance of $\boldsymbol{\gamma} \in \mathcal{H}$ ), we have:

$$
\begin{aligned}
\mathrm{F}_{\boldsymbol{u} \star}: T_{\phi} \Phi & \rightarrow T_{\phi^{u}} \mathcal{M} \\
\boldsymbol{X}_{\phi} & \mapsto \mathrm{F}_{\boldsymbol{u} \star} \boldsymbol{X}_{\phi}=\rho(\boldsymbol{u})^{-1}\left(X(\phi)+\delta_{\boldsymbol{d u}_{\mid \phi}^{-1}\left(\boldsymbol{X}_{\phi}\right)} \phi\right) \frac{\delta}{\delta[\phi]},
\end{aligned}
$$

Dually, the pullback application allows to realise basic forms on $\Phi$,

$$
\begin{aligned}
\mathrm{F}_{u}^{\star}: \Omega^{\bullet}(\mathcal{M}) & \rightarrow \Omega_{\text {basic }}^{\bullet}(\Phi) \\
\overline{\boldsymbol{\alpha}}_{\mid[\phi]} & \mapsto \mathrm{F}_{u}^{\star} \overline{\boldsymbol{\alpha}}_{\mid \phi}=: \boldsymbol{\alpha}_{\mid \phi}^{u} .
\end{aligned}
$$

Indeed, as $\mathrm{F}_{\boldsymbol{u}} \sim \pi, \Omega_{\text {basic }}^{\bullet}(\Phi)=\operatorname{Im} \pi^{\star} \simeq \operatorname{ImF}_{\boldsymbol{u}}^{\star}$. The $\mathcal{H}$-basicity of $\boldsymbol{\alpha}^{\boldsymbol{u}}$ is easily proven: $R_{\gamma}^{\star} \boldsymbol{\alpha}^{u}=R_{\gamma}^{\star} \mathrm{F}_{u}^{\star} \overline{\boldsymbol{\alpha}}=\mathrm{F}_{\boldsymbol{u}}^{\star} \overline{\boldsymbol{\alpha}}=\boldsymbol{\alpha}^{u}$, with $\gamma \in \mathcal{H}$, and $\boldsymbol{\alpha}^{u}\left(\chi^{v}\right)=\left(\mathrm{F}_{\boldsymbol{u}}^{\star} \overline{\boldsymbol{\alpha}}\right)\left(\chi^{v}\right)=\overline{\boldsymbol{\alpha}}\left(\mathrm{F}_{\boldsymbol{u} \star} \chi^{v}\right)=0$. The $\mathcal{H}$-invariance ensues, $\left(\boldsymbol{\alpha}^{u}\right)^{\gamma}=\boldsymbol{\alpha}^{u}$ for $\gamma \in \mathcal{H}$.

As it stands, $\boldsymbol{\alpha}^{\boldsymbol{u}}=\mathrm{F}_{u}^{\star} \overline{\boldsymbol{\alpha}}$ is the basic counterpart of $\overline{\boldsymbol{\alpha}} \in \Omega^{\bullet}(\mathcal{M})$. But as the notation suggests, we would rather like to see $\boldsymbol{\alpha}^{u}$ as the basic version of some given form $\boldsymbol{\alpha} \in \Omega^{\bullet}(\Phi)$ whose functional expression could presumably be given in terms of $\boldsymbol{\alpha}$ and $\boldsymbol{u}$. This is indeed possible via a shift of viewpoint: one may notice that to a given $\boldsymbol{\alpha}_{\mid \phi}=\alpha(\wedge \bullet \boldsymbol{d} \phi ; \phi) \in \Omega^{\bullet}(\Phi)$ corresponds $\overline{\boldsymbol{\alpha}}_{\mid[\phi]}=\alpha\left(\wedge^{\bullet} \boldsymbol{d}[\phi] ;[\phi]\right) \in \Omega^{\bullet}(\mathcal{M})$ built via the same functional $\alpha(;)$ (the only difference being the type of arguments the latter takes in). Now we can define the dressed version, or dressing, of $\boldsymbol{\alpha}$ via (2.47) as being

$$
\boldsymbol{\alpha}_{\mid \phi}^{\boldsymbol{u}}:=\mathrm{F}_{\boldsymbol{u}}^{\star} \overline{\boldsymbol{\alpha}}_{\mid \phi}=\alpha\left(\wedge^{\bullet} \mathrm{F}_{\boldsymbol{u}}^{\star} \boldsymbol{d}[\phi] ; \mathrm{F}_{\boldsymbol{u}}(\phi)\right)=\alpha\left(\wedge^{\bullet} \boldsymbol{d} \phi^{\boldsymbol{u}} ; \phi^{\boldsymbol{u}}\right) \quad \in \Omega_{\text {basic }}^{\bullet}(\Phi),
$$

where we have defined the basic basis 1 -form $\boldsymbol{d} \phi^{\boldsymbol{u}}:=\mathrm{F}_{u}^{\star} \boldsymbol{d}[\phi] \in \Omega_{\text {basic }}^{1}(\Phi)$, with $\boldsymbol{d}[\phi] \in$ $\Omega^{1}(\mathcal{M})$ basis of forms on $\mathcal{M}$. As per our stated desiderata, the latter can be written explicitly, via (2.46), in terms of $\boldsymbol{d} \phi$ and $\boldsymbol{u}$ :

$$
\begin{aligned}
\boldsymbol{d} \phi_{\mid \phi}^{\boldsymbol{u}}\left(\boldsymbol{X}_{\phi}\right) & :=\left(\mathrm{F}_{\boldsymbol{u}}^{\star} \boldsymbol{d}[\phi]_{\mid[\phi]}\right)\left(\boldsymbol{X}_{\phi}\right)=\boldsymbol{d}[\phi]_{\mid[\phi]}\left(\mathrm{F}_{\boldsymbol{u} \star} \boldsymbol{X}_{\phi}\right)=\rho(\boldsymbol{u})^{-1}\left(X(\phi)+\delta_{\boldsymbol{d u u}_{\mid \phi}^{-1}\left(\boldsymbol{X}_{\phi}\right)} \phi\right) \\
& =\rho(\boldsymbol{u})^{-1}\left(\boldsymbol{d} \phi_{\mid \phi}\left(\boldsymbol{X}_{\phi}\right)+\delta_{\boldsymbol{d u u}_{\mid \phi}^{-1}\left(\boldsymbol{X}_{\phi}\right)} \phi\right)=\left[\rho(\boldsymbol{u})^{-1}\left(\boldsymbol{d} \phi+\delta_{\left.\boldsymbol{d u u ^ { - 1 }} \phi\right)}\right]_{\mid \phi}\left(\boldsymbol{X}_{\phi}\right),\right.
\end{aligned}
$$

that is $\quad \boldsymbol{d} \phi^{\boldsymbol{u}}=\rho(\boldsymbol{u})^{-1}\left(\boldsymbol{d} \phi+\delta_{\boldsymbol{d u u ^ { - 1 }}} \phi\right)=\left\{\begin{array}{c}\boldsymbol{d} A^{\boldsymbol{u}}=\boldsymbol{u}^{-1}\left(\boldsymbol{d} A+D\left\{\boldsymbol{d} \boldsymbol{u} \boldsymbol{u}^{-1}\right\}\right) \boldsymbol{u} \\ \boldsymbol{d} \varphi^{\boldsymbol{u}}=\rho(\boldsymbol{u})^{-1}\left(\boldsymbol{d} \varphi-\rho_{*}\left(\boldsymbol{d} \boldsymbol{u} \boldsymbol{u}^{-1}\right) \varphi\right)\end{array}\right.$

\footnotetext{
${ }^{12}$ Again, we here work with the simplifying assumption of a $H$-dressing. But the following can be adapted with minor adjustments to $\Phi$-dependent $K$-dressing fields $\boldsymbol{u}: \Phi \rightarrow \mathcal{D} r[K, G]$, s.t. $R_{\gamma}^{\star} \boldsymbol{u}=\gamma^{-1} \boldsymbol{u}$ for $\gamma \in \mathcal{K} \subset \mathcal{H}$, leaving then residual $\mathcal{J}$-gauge transformations (called residual transformations of the first kind in [33]).
} 
Comparing this to (2.7), we see that due to the formal similarity between $\mathrm{F}_{\boldsymbol{u}}$ and $\boldsymbol{\Psi}$, their actions are formally alike. This generalises to $\boldsymbol{\alpha}$ above. Indeed, given that its $\mathcal{H}$-gauge transformation is

$$
\boldsymbol{\alpha}_{\mid \phi}^{\gamma}:=\Psi^{\star} \boldsymbol{\alpha}_{\mid \phi}=\alpha\left(\wedge^{\bullet} \boldsymbol{\Psi}^{\star} \boldsymbol{d} \phi ; \boldsymbol{\Psi}(\phi)\right)=\alpha\left(\wedge^{\bullet} \boldsymbol{d} \phi^{\gamma} ; \phi^{\gamma}\right),
$$

by comparison with (2.48) we see that the general rule of thumb to obtain the dressed version $\boldsymbol{\alpha}^{\boldsymbol{u}} \in \Omega_{\mathrm{basic}}^{\bullet}(\Phi)$ of $\boldsymbol{\alpha} \in \Omega^{\bullet}(\Phi)$ is to replace $\boldsymbol{\gamma} \rightarrow \boldsymbol{u}$ in $\boldsymbol{\alpha}^{\gamma}{ }^{13}$

Seing now (2.48) as a form on the $\mathcal{G}$-bundle of dressed fields $\Phi^{\boldsymbol{u}}$, the $\mathcal{G}$-transformation of $\boldsymbol{\alpha}^{\boldsymbol{u}}$ is obtained in exactly the same way as the $\mathcal{H}$-transformation of $\boldsymbol{\alpha}$ on $\Phi$. We thus obtain $\left(\boldsymbol{\alpha}^{\boldsymbol{u}}\right)^{\boldsymbol{\xi}}$ by replacing $\boldsymbol{\alpha} \rightarrow \boldsymbol{\alpha}^{\boldsymbol{u}}$ and $\boldsymbol{\gamma} \rightarrow \boldsymbol{\xi}$ in the formula for $\boldsymbol{\alpha}^{\gamma}$. Both $\boldsymbol{\alpha}^{\boldsymbol{u}}$ and $\left(\boldsymbol{\alpha}^{\boldsymbol{u}}\right)^{\boldsymbol{\xi}}$ are basic forms (corresponding to $\alpha$ ) on the $\mathcal{H}$-bundle $\Phi$.

Taking the example of $\boldsymbol{d} \phi^{\boldsymbol{u}}$, in analogy with (2.5)-(2.6), we have $R_{\xi}^{\star} \boldsymbol{d} \phi^{\boldsymbol{u}}=\rho(\xi)^{-1} \boldsymbol{d} \phi^{\boldsymbol{u}}$ and $\boldsymbol{d} \phi_{\mid \phi^{u}}^{\boldsymbol{u}}\left(\zeta_{\phi^{u}}^{v}\right)=\delta_{\zeta} \phi^{\boldsymbol{u}}$ (the infinitesimal version of eq. (2.42)), where $\zeta^{v} \in \Gamma\left(V \Phi^{\boldsymbol{u}}\right)$ and $\zeta \in$ Lie $\mathcal{G} .{ }^{14}$ So, by the same computation leading to (2.7), the $\mathcal{G}$-transformation of $\boldsymbol{d} \phi^{\boldsymbol{u}}$ is

$$
\left(\boldsymbol{d} \phi^{\boldsymbol{u}}\right)^{\boldsymbol{\xi}}=\rho(\boldsymbol{\xi})^{-1}\left(\boldsymbol{d} \phi^{\boldsymbol{u}}+\delta_{\boldsymbol{d} \xi \boldsymbol{\xi}^{-1}} \phi^{\boldsymbol{u}}\right)=\left\{\begin{array}{c}
\left(\boldsymbol{d} A^{\boldsymbol{u}}\right)^{\xi}=\boldsymbol{\xi}^{-1}\left(\boldsymbol{d} A^{\boldsymbol{u}}+D^{A^{u}}\left\{\boldsymbol{d} \boldsymbol{\xi} \boldsymbol{\xi}^{-1}\right\}\right) \boldsymbol{\xi} \\
\left(\boldsymbol{d} \varphi^{\boldsymbol{u}}\right)^{\boldsymbol{\xi}}=\rho(\boldsymbol{\xi})^{-1}\left(\boldsymbol{d} \varphi^{\boldsymbol{u}}-\rho_{*}\left(\boldsymbol{d} \boldsymbol{\xi} \boldsymbol{\xi}^{-1}\right) \varphi^{u}\right)
\end{array}\right.
$$

This result, together with eq. (2.42), allows to cross-check algebraically the $\mathcal{G}$ transformation of $\boldsymbol{\alpha}^{u}$, which is

$$
\left(\boldsymbol{\alpha}^{\boldsymbol{u}}\right)^{\boldsymbol{\xi}}=\alpha\left(\wedge^{\bullet}\left(\boldsymbol{d} \phi^{\boldsymbol{u}}\right)^{\boldsymbol{\xi}} ;\left(\phi^{\boldsymbol{u}}\right)^{\boldsymbol{\xi}}\right) .
$$

To repeat, given the functional properties of $\alpha$, the latter is formally identical to the $\mathcal{H}$ transformation of $\boldsymbol{\alpha}$. In particular, for $\boldsymbol{\alpha}^{\boldsymbol{u}} \in \Omega_{\text {inv }}^{1}\left(\Phi^{\boldsymbol{u}}\right)$, eq. (2.52) specialises as

$$
\begin{aligned}
\left(\boldsymbol{\alpha}^{\boldsymbol{u}}\right)^{\xi} & =\alpha\left(\boldsymbol{d} \phi^{\boldsymbol{u}}+\delta_{\boldsymbol{d} \xi \xi^{-1}} \phi^{\boldsymbol{u}} ; \phi^{\boldsymbol{u}}\right) \\
& =\boldsymbol{\alpha}^{\boldsymbol{u}}+\alpha\left(\delta_{\boldsymbol{d} \xi \xi^{-1}} \phi^{\boldsymbol{u}} ; \phi^{\boldsymbol{u}}\right) .
\end{aligned}
$$

Reverting back to the original viewpoint, equations (2.51)-(2.53) can also be seen as relations between forms on the initial $\mathcal{H}$-bundle $\Phi$. Seing that indeed (2.51) is rewritten as

$$
\left(\boldsymbol{d} \phi^{\boldsymbol{u}}\right)^{\boldsymbol{\xi}}=\rho(\boldsymbol{\xi})^{-1}\left(\boldsymbol{d} \phi^{\boldsymbol{u}}+\rho(u)^{-1} \delta_{\left.\boldsymbol{u} \boldsymbol{\xi} \xi \boldsymbol{\xi}^{-1} \boldsymbol{u}^{-1} \phi\right)} \in \Omega_{\text {basic }}^{1}(\Phi),\right.
$$

so that for $\boldsymbol{\alpha}=\alpha(\boldsymbol{d} \phi ; \phi) \in \Omega_{\text {inv }}^{1}(\Phi),(2.53)$ is also

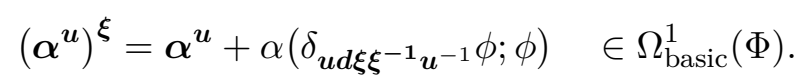

One may then notice the striking similarity between (2.55) and eq.(2.30) reflecting the ambiguity in building basic forms from variational Ehresmann connections. This is no coincidence, as we clarify in the following section concluding this review of the DFM.

\footnotetext{
${ }^{13}$ The latter being obtained, as we've seen, either geometrically via the equivariance and verticality properties of $\boldsymbol{\alpha}$, or algebraically by inserting (2.7) in its functional expression as suggested by (2.50).

${ }^{14}$ These can be taken as axiomatic on $\Phi^{u}$, or can motivated by reference to $\Phi$ : since by assumption $\phi^{\xi}=\phi$, we can formally admit $\boldsymbol{d} \phi\left(\zeta^{v}\right)=0$, and since by definition $\boldsymbol{u}^{\xi}=\boldsymbol{u} \xi$, infinitesimally we can formally admit $\boldsymbol{d} \boldsymbol{u}\left(\zeta^{v}\right)=\zeta^{v}(\boldsymbol{u})=\boldsymbol{u} \zeta$. By the definition (2.49) of $\boldsymbol{d} \phi^{\boldsymbol{u}}$, its $\mathcal{G}$-equivariance is clear, while it is easy to show explicitly that $\boldsymbol{d} \phi_{\mid \phi^{u}}^{\boldsymbol{u}}\left(\zeta_{\phi^{u}}^{v}\right)=\rho(\boldsymbol{u})^{-1} \delta_{\boldsymbol{u} \zeta \boldsymbol{u}^{-1}} \phi=\delta_{\zeta} \phi^{\boldsymbol{u}}$.
} 


\subsubsection{Field-dependent dressing fields and variational connections}

Flat Ehresmann variational connections. By definition of a field-dependent dressing field, we have for $\gamma \in \mathcal{H}$ and $\chi_{\phi}^{v} \in V_{\phi} \Phi$ :

$$
\begin{aligned}
& R_{\gamma}^{\star}\left(-\boldsymbol{d u} \boldsymbol{u}^{-1}\right)=-\boldsymbol{d}\left(R_{\gamma}^{\star} \boldsymbol{u}\right) R_{\gamma}^{\star} \boldsymbol{u}^{-1}=\gamma^{-1}(-\boldsymbol{d u u})^{-1} \gamma, \\
& -\boldsymbol{d} \boldsymbol{u} \boldsymbol{u}_{\mid \phi}^{-1}\left(\chi_{\phi}^{v}\right)=-\left(\chi^{v} \boldsymbol{u}\right)(\phi) \boldsymbol{u}(\phi)^{-1}=+\chi \boldsymbol{u}(\phi) \boldsymbol{u}(\phi)^{-1}=\chi \in \text { Lie } \mathcal{H} .
\end{aligned}
$$

As it is furthermore clear that $\boldsymbol{d}\left(-\boldsymbol{d u} \boldsymbol{u}^{-1}\right)+\frac{1}{2}\left[\boldsymbol{d} \boldsymbol{u} \boldsymbol{u}^{-1}, \boldsymbol{d u} \boldsymbol{u}^{-1}\right] \equiv 0$, the quantity $\stackrel{\circ}{\boldsymbol{\omega}}:=$ $-\boldsymbol{d} \boldsymbol{u} \boldsymbol{u}^{-1}=\boldsymbol{u} \boldsymbol{d} \boldsymbol{u}^{-1}$ is thus a flat variational Ehresmann connection, $\stackrel{\Omega}{\Omega}=0$.

The same is true of $\stackrel{\circ}{\boldsymbol{\omega}}^{\prime}:=-\boldsymbol{d} \boldsymbol{u}^{\prime} \boldsymbol{u}^{\prime-1}=\boldsymbol{u}^{\prime} \boldsymbol{d} \boldsymbol{u}^{\prime-1}$ with $\boldsymbol{u}^{\prime}=\boldsymbol{u}^{\boldsymbol{\xi}}=\boldsymbol{u} \boldsymbol{\xi}$, since by definition $R_{\gamma}^{\star} \boldsymbol{\xi}=\boldsymbol{\xi}$. From this also follows that $\stackrel{\circ}{\boldsymbol{\omega}}^{\prime}=\stackrel{\circ}{\boldsymbol{\omega}}+\stackrel{\circ}{\boldsymbol{\beta}}$, where $\stackrel{\circ}{\boldsymbol{\beta}}:=-\boldsymbol{u d} \boldsymbol{\xi} \boldsymbol{\xi}^{-\mathbf{1}} \boldsymbol{u}^{-1} \in \Omega_{\text {tens }}^{1}(\Phi)$ since

$$
\begin{aligned}
R_{\gamma}^{\star} \stackrel{\circ}{\boldsymbol{\beta}} & =\gamma^{-1}\left(-\boldsymbol{u d} \boldsymbol{\xi} \boldsymbol{\xi}^{-\mathbf{1}} \boldsymbol{u}^{-1}\right) \gamma=\gamma^{-1} \stackrel{\circ}{\boldsymbol{\beta}} \gamma, \\
\stackrel{\boldsymbol{\beta}}{(}\left(\chi^{v}\right) & =-\boldsymbol{u d} \boldsymbol{\xi}\left(\chi^{v}\right) \boldsymbol{\xi}^{-1} \boldsymbol{u}^{-1}=0 .
\end{aligned}
$$

Therefore, the existence of a field-dependent dressing field $\boldsymbol{u}$ is equivalent to the existence of a flat variational Ehresmann connection $\stackrel{\dot{\omega}}{\omega}$ on field space $\Phi$, and the a priori ambiguity in choosing/building such a dressing field $\left(\boldsymbol{u}^{\prime} / \boldsymbol{u}\right)$ translates as an ambiguity (assuming a specific form, $\stackrel{\leftrightarrow}{\boldsymbol{\beta}}$ having the form it has) in picking a choice within the affine space of flat connections. It is thus not surprising to find some similarities in the way basic form are built via dressing and via non-flat connections. Yet, the differences are also worth stressing.

Using $\stackrel{\circ}{\omega}$ we could perform the horizontalisation procedure seen in section 2.2.1, with in particular

$$
\boldsymbol{d} \phi_{\grave{\omega}}^{h}=\boldsymbol{d} \phi-\delta_{\dot{\omega}} \phi \in \Omega_{\mathrm{hor}}^{1}(\Phi)
$$

as a special case of eq. (2.20). Then, we have that eq. (2.49) is rewritten as

$$
\boldsymbol{d} \phi^{\boldsymbol{u}}=\rho(\boldsymbol{u})^{-1} \boldsymbol{d} \phi_{\grave{\omega}}^{h}=\rho(\boldsymbol{u})^{-1}\left(\boldsymbol{d} \phi-\delta_{\grave{\omega}} \phi\right) \quad \in \Omega_{\mathrm{basic}}^{1}(\Phi),
$$

Through this simplest example, we see on display the crucial difference between the DFM and the horizontalisation via connection: the dressing operation takes care not only of horizontalisation, via the term $\delta_{\dot{\omega}} \phi$, but also of trivialising the equivariance via the term $\rho(\boldsymbol{u})^{-1}$. Hence, while one can use Ehresmann connections to produce basic forms out of invariant forms only, one can use the DFM to produce basic forms out of forms of any equivariance. ${ }^{15}$

As to the matter of ambiguity, eq. (2.54) is

$$
\left(\boldsymbol{d} \phi^{u}\right)^{\boldsymbol{\xi}}=\rho(\boldsymbol{\xi})^{-1}\left(\boldsymbol{d} \phi^{\boldsymbol{u}}+\rho(u)^{-1} \delta_{\boldsymbol{\beta}} \phi\right) \quad \in \Omega_{\text {basic }}^{1}(\Phi),
$$

which is close to eq. (2.29) but not quite the same. The difference being again only in the equivariance, i.e. the presence of $\rho(\boldsymbol{\xi})$ and $\rho(\boldsymbol{u})$. But in the case of $\boldsymbol{\alpha}=\alpha(\boldsymbol{d} \phi ; \phi) \in \Omega_{\text {inv }}^{1}(\Phi)$, the latter disappear so eq. (2.55) rewritten as

$$
\left(\boldsymbol{\alpha}^{\boldsymbol{u}}\right)^{\boldsymbol{\xi}}=\boldsymbol{\alpha}^{\boldsymbol{u}}-\alpha\left(\delta_{\dot{\boldsymbol{\beta}}} \phi ; \phi\right) \quad \in \Omega_{\mathrm{basic}}^{1}(\Phi)
$$

is indeed seen to be special case of (2.30), with $\left(\boldsymbol{\alpha}^{\boldsymbol{u}}\right)^{\boldsymbol{\xi}}=\boldsymbol{\alpha}_{\dot{\omega}^{\prime}}^{b}$ and $\boldsymbol{\alpha}^{\boldsymbol{u}}=\boldsymbol{\alpha}_{\dot{\omega}}^{b}$.

\footnotetext{
${ }^{15}$ Twisted equivariant forms included.
} 
When considering the construction of basic forms out of invariant forms, as will be our main concerns ahead w.r.t. invariant gauge theories, one may thus expect convergence between the formal results one obtains from either variational Ehresmann connections or the DFM. But the respective merits of both approaches must be parsed and kept in mind.

One the one hand, the existence of a field-dependent dressing field, thus of a flat connection, is likely a strong constraint on the topology of field space $\Phi .{ }^{16}$ There is then no guarantee that it will always be possible to find/build such a dressing field globally defined across $\Phi$ (Gribov-Singer-like obstructions may exist). On the contrary, $\Phi$ can always be endowed with a non-flat Ehresmann connection, which imposes no such topological constraint.

On the other hand, the ambiguity of choice among the affine space of connections generally cannot be associated with some underlying useful symmetry transformations. Especially so if a connection comes from a natural bundle metric (as e.g. on $\mathcal{A}$ ), so that this ambiguity could arguably be discarded. Whereas, as we have remarked already, the group $\mathcal{G}$ controlling the ambiguity in the choice/building of dressing fields may be a physically relevant symmetry to which potentially observable charges can be associated.

There is no telling in advance which method to choose, as much will depend on the specific examples under consideration. If a bundle metric exists, then it is natural to use the associated connection. If a dressing field is readily identified, one should use the full power of the DFM. In section 4, we will nonetheless give the most general form, according to both scheme, of the basic presymplectic structure for invariant gauge theories. Before that, we say a final word about how dressing fields may also give rise to twisted variational connections.

Flat twisted variational connections. Considering again a field-dependent dressing field $\boldsymbol{u}: \Phi \rightarrow \mathcal{D r}[H, G]$ and a 1-cocycle $C$, we define a twisted dressing field $C(\boldsymbol{u})$ by $C(\boldsymbol{u})(\phi):=C(\phi, \boldsymbol{u}(\phi))$. Due to the cocycle defining property we indeed have, for $\gamma \in \mathcal{H}$,

$$
\begin{aligned}
{\left[R_{\gamma}^{\star} C(\boldsymbol{u})\right](\phi) } & =C\left(\phi^{\gamma}, \boldsymbol{u}\left(\phi^{\gamma}\right)\right)=C\left(\phi^{\gamma}, \gamma^{-1} \boldsymbol{u}(\phi)\right) \\
& =C\left(\phi^{\gamma}, \gamma\right) C(\phi, \gamma \boldsymbol{u}(\phi))=C(\phi, \gamma)^{-1} C(\phi, \gamma \boldsymbol{u}(\phi)), \\
& =\left[C(, \gamma)^{-1} C(\boldsymbol{u})\right](\phi) .
\end{aligned}
$$

The infinitesimal version of which is

$$
\boldsymbol{L}_{\chi^{v}} C(\boldsymbol{u})=\iota_{\chi^{v}} \boldsymbol{d} C(\boldsymbol{u})=-\left.\frac{d}{d \tau} C(, \exp \tau \chi)\right|_{\tau=0} C(\boldsymbol{u}),
$$

with $\chi^{v} \in \Gamma(V \Phi)$.

In the same manner that $\boldsymbol{u}$ defines a flat variational Ehresmann connection $\stackrel{\dot{\omega}}{\text {, via }}$ the cocycle it defines a flat twisted variational connection $\stackrel{\circ}{\varpi}=-\boldsymbol{d} C(\boldsymbol{u}) C(\boldsymbol{u})^{-1}=$ $C(\boldsymbol{u}) \boldsymbol{d} C(\boldsymbol{u})^{-1}$. From the above follows indeed easily,

$$
\begin{aligned}
& R_{\gamma}^{\star} \stackrel{\circ}{\varpi}_{\mid \phi^{\gamma}}=C(\phi, \gamma)^{-1} \stackrel{\circ}{\varpi}_{\mid \phi} C(\phi, \gamma)+C(\phi, \gamma)^{-1} \boldsymbol{d} C(, \gamma)_{\mid \phi}, \\
& \stackrel{\circ}{\varpi}_{\mid \phi}\left(\chi_{\phi}^{v}\right)=\boldsymbol{d} C(\boldsymbol{u}) C(\boldsymbol{u})^{-1}\left(\chi^{v}\right)=+\left.\frac{d}{d \tau} C(, \exp \tau \chi)\right|_{\tau=0},
\end{aligned}
$$

\footnotetext{
${ }^{16}$ In the finite dimensional case, a principal bundle over a connected manifold can have a flat connection only if it is trivial!
} 
the defining axioms $(2.32)-(2.33)$ of a twisted connection. Clearly, $\boldsymbol{d} \stackrel{\circ}{\varpi}+\frac{1}{2}[\stackrel{\circ}{\varpi}, \stackrel{\circ}{\varpi}]=0$.

In section 2.2.2, we saw that a general non-invariant Lagrangian is $R_{\gamma}^{\star} L=L+c(, \gamma)$, with classical gauge anomaly $\boldsymbol{L}_{\chi^{v}} L=\left.\frac{d}{d \tau} c(, \exp \tau \chi)\right|_{\tau=0}=: \alpha(\chi, \quad)$. It is associated with the twisted functional $Z:=\exp i \int L \in \Omega_{\text {tens }}^{0}(\Phi, \mathbb{C})^{C}$ s.t. $R_{\gamma}^{\star} Z=C(, \gamma)^{-1} Z$ with $C(, \gamma):=$ $\exp -i \int c(, \gamma) \in \mathrm{U}(1)$.

Admitting a dressing field exists, the corresponding flat twisted connection is $\stackrel{\circ}{\varpi}=$ $i \boldsymbol{d} \int c(\boldsymbol{u}):=i \boldsymbol{d} \int c(, \boldsymbol{u})$. The associated twisted covariant derivative of $Z$ is thus,

$$
\boldsymbol{D}^{\stackrel{\circ}{\varpi}} Z=\boldsymbol{d} Z+\stackrel{\circ}{\varpi} Z=\left(i \boldsymbol{d} \int L+c(\boldsymbol{u})\right) Z \quad \in \Omega_{\text {tens }}^{1}(\Phi, \mathbb{C})^{C} .
$$

We have then that $L+c(\boldsymbol{u}) \in \Omega_{\text {basic }}^{0}(\Phi)$. This is none other than a Wess-Zumino improved (i.e. $\mathcal{H}$-invariant) Lagrangian, and $c(\boldsymbol{u})$ is a Wess-Zumino functional which (usually by special design) satisfies $\boldsymbol{L}_{\chi^{v}} c(\boldsymbol{u})=-\alpha(\chi, \quad)$.

A WZ functional is thus seen to be the pre-potential of a flat twisted connection. Any twisted connection $\tilde{\boldsymbol{\omega}}$ adapted to this context (i.e. fit to induce a twisted covariant derivative) satisfies $\tilde{\boldsymbol{\omega}}\left(\chi^{v}\right)=-i \int \alpha(\chi, \quad)$ - see eq. (2.35). Here, we have indeed $\stackrel{\circ}{\varpi}\left(\chi^{v}\right)=$ $i \int \boldsymbol{d} c(\boldsymbol{u})\left(\chi^{v}\right)=i \int \boldsymbol{L}_{\chi^{v}} c(\boldsymbol{u})=-i \int \alpha(\chi, \quad)$ as a special case of (2.61).

Let us finally remark that the above improved Lagrangian is precisely what is immediately given by application of the DFM: the dressed version of the non-invariant Lagrangian $L \in \Omega_{\mathrm{eq}}^{0}(\Phi)^{C}$ is

$$
\begin{aligned}
L^{\boldsymbol{u}}(\phi) & :=\left(\mathrm{F}_{\boldsymbol{u}}^{\star} L\right)(\phi)=L\left(\mathrm{~F}_{\boldsymbol{u}}(\phi)\right)=L\left(\phi^{\boldsymbol{u}}\right)=L(\phi)+c(\phi, \boldsymbol{u}(\phi)), \\
\hookrightarrow \quad L^{\boldsymbol{u}} & =L+c(\boldsymbol{u}) \in \Omega_{\text {basic }}^{0}(\Phi) .
\end{aligned}
$$

This illustrates a commentary we made in the previous subsection, to the effect that the DFM can associate basic forms to forms of any equivariance, including twisted equivariance. This was extensively used in [33], section 5.3.2, to produce the basic (dressed) presymplectic structure of non-invariant pure gauge theories.

After these long technical preliminaries, we are ready for our main application concerning the presymplectic structure of invariant matter coupled gauge theories.

\section{Presymplectic structures of matter coupled gauge theories over bounded regions}

In this section we first briefly remind one original aim of the covariant phase space formalism for gauge field theory, and the impediment posed by the presence of boundaries: that is the boundary problem. Then we provide results of some generality about the presymplectic structure of invariant matter coupled gauge theories. Some of these are necessary to show how one may try to answer the boundary problem by constructing basic presymplectic structures obtained either via the DFM or via variational connections.

\subsection{Covariant phase space formalism}

As already evoked just before section 2.2, a gauge theory is specified by a Lagrangian functional $L: \Phi \rightarrow \Omega^{n}(\mathcal{P}, \mathbb{R}), \phi \mapsto L(\phi)=L(A, \varphi), n$ the dimension of spacetime $M$. For 
the associated action $S=\int_{U} L$ to be finite, ${ }^{17}$ one usually assumes the region $U \subset M$ to be compact or closed, or that the fields are either compactly supported or satisfy sufficiently fast fall-off conditions at infinity (which amounts to an effective compactification of $\mathcal{M}$ ).

The variational principle stipulates that the field equations are found from requiring $S$ to be stationary, $\delta S=0 \forall \delta \phi$, under well-defined boundary conditions. In the formulation adopted here this translates as $\boldsymbol{d} S(\boldsymbol{X})=0 \forall \boldsymbol{X} \in \Gamma(T \Phi)$, i.e the functional $S: \Phi \rightarrow \mathbb{R}$ is closed, $\boldsymbol{d} S=0$. Admitting that $\boldsymbol{d}$ and $\int$ commute, this gives

$$
\boldsymbol{d} S=\int_{U} \boldsymbol{d} L=\int_{U} \boldsymbol{E}+d \boldsymbol{\theta}=\int_{U} \boldsymbol{E}+\int_{\partial U} \boldsymbol{\theta}=0
$$

where $\boldsymbol{E}_{\mid \phi}=E(\boldsymbol{d} \phi ; \phi)$ is the field equations 1-form and $\boldsymbol{\theta}_{\mid \phi}=\theta(\boldsymbol{d} \phi ; \phi)$ is the presymplectic potential current 1-form. Here $E$ and $\theta$ are different functionals of $\phi$, both linear in $\boldsymbol{d} \phi$ (nonetheless based on the same functional as $L$ ).

The point of the covariant phase space approach, or covariant Hamiltonian formalism, is to associate a phase space equipped with a symplectic form to a field theory over a region $U \subseteq \mathcal{M}$, and doing so while keeping all spacetimes symmetries manifest. Such a symplectic structure would be the starting point for a canonical or geometric quantization procedure, or so was one original motivation. Some attribute the inception of the idea to [1-3], but it actually goes further back and has close ties to the multisymplectic formalism as shown in [56], which we recommend. Classical references are [57, 58], and modern introductions are [59, 60] (see also [61] for a compact summary). The recent review [4] gives historical context and shows the relation of covariant phase space methods to other approaches such as the multisymplectic formalism and the variational bicomplex.

The configuration space is the field space, here the $\mathcal{H}$-bundle $\Phi$. The covariant phase space is the solution space $\mathcal{S}$ - the shell - defined by $\boldsymbol{E}=0$. The physical, or reduced, phase space is $\mathcal{S} / \mathcal{H}=: \mathcal{M}_{\mathcal{S}}$ if it can be endowed with a well-defined symplectic 2 -form. Notice then that the Hamiltonian flow belongs to the physical transformation group $\operatorname{Diff}(\mathcal{M})$ in the SES (2.1), and the corresponding Hamiltonian vector field thus belongs to $\Gamma(T \mathcal{M})$ in the SES (2.2).

The presymplectic potential $\boldsymbol{\theta}$ allows to define the Noether currents and charges associated with the action of $\mathcal{H}$, and a natural candidate symplectic form is derived from it. Since $[\boldsymbol{d}, d]=0$, we have $0 \equiv \boldsymbol{d}^{2} L=\boldsymbol{d} \boldsymbol{E}+d(\boldsymbol{d} \boldsymbol{\theta})$. So, the 2 -form $\boldsymbol{\Theta}:=\boldsymbol{d} \boldsymbol{\theta}$ is $d$-closed on-shell, $d \boldsymbol{\Theta}=0_{\mid \mathcal{S}}$. Given a codimension 1 submanifold $\Sigma \subset U$, we have $\boldsymbol{\Theta}_{\Sigma}:=\int_{\Sigma} \boldsymbol{\Theta} \in \Omega^{2}(\Phi, \mathbb{R})$. The presymplectic potential is $\boldsymbol{\theta}_{\Sigma}:=\int_{\Sigma} \boldsymbol{\theta} \in \Omega^{1}(\Phi, \mathbb{R})$, so that $\boldsymbol{\Theta}_{\Sigma}=\boldsymbol{d} \boldsymbol{\theta}_{\Sigma}$. Since $\boldsymbol{d} \boldsymbol{\Theta}_{\Sigma}=0$, $\boldsymbol{\Theta}_{\Sigma}$ is a presymplectic 2-form (hence the name given to $\boldsymbol{\theta}$ and $\boldsymbol{\theta}_{\Sigma}$ ). It allows to define a Poisson bracket between charges.

For $\boldsymbol{\theta}_{\Sigma}$ and $\boldsymbol{\Theta}_{\Sigma}$ to induce a symplectic structure on $\mathcal{S} / \mathcal{H}=\mathcal{M}_{\mathcal{S}}$, they must be basic on $\Phi$. This requires that on-shell the $\mathcal{H}$-equivariance and verticality properties of $\boldsymbol{\theta}$ and $\Theta$ are right, i.e. that adequate boundary conditions are specified. This last requirement is jeopardised when considering bounded regions, or when one considers the partitioning

\footnotetext{
${ }^{17}$ Since we took the viewpoint that $L$ is a n-form on $\mathcal{P}$, actually $S=\int_{\sigma(U)} L=\int_{U} \sigma^{*} L$ with $\sigma: U \rightarrow \mathcal{P}$ a local section and $\sigma^{*} L$ written in terms of the gauge potential $\sigma^{*} A$. For convenience, we shall omit $\sigma$ in writing integration domains.
} 
a region into subregions sharing a fictitious boundary. This is what we call the boundary problem: the obstruction to the basicity of $\boldsymbol{\theta}_{\Sigma}$ and $\boldsymbol{\Theta}_{\Sigma}$ due a boundary $\partial \Sigma$.

Before considering potential answers to the boundary problem in section 4 , we want to give general results on the presymplectic structure of invariant gauge theories. Precisely, we are interested in identifying the Noether currents and charges, the Poisson bracket of charges, and most importantly the $\mathcal{H}$-gauge transformations of $\boldsymbol{\theta}_{\Sigma}$ and $\boldsymbol{\Theta}_{\Sigma}$. Whenever possible we will give these results as functions of the field equations $\boldsymbol{E}$ so that on-shell restrictions are read-off immediately.

\subsection{Presymplectic structure of invariant matter coupled gauge theories}

We are concerned with gauge theories that strictly respect the gauge principle, so that $L \in \Omega_{\text {basic }}^{0}(\Phi)$ (case 1 mentioned just before section 2.2). As $\boldsymbol{d}$ is a covariant derivative on basic forms, we know immediately that $\boldsymbol{d} L \in \Omega_{\text {basic }}^{1}(\Phi)$.

In the pure gauge case, $L \in \Omega_{\text {basic }}^{0}(\mathcal{A})$, the Utiyama theorem $[62,63]$ states that $L$ must factorise through the curvature map, $L=\tilde{L} \circ F$, with $\tilde{L}$ a $\operatorname{Ad}(H)$-invariant functional on $\Omega_{\text {tens }}^{\bullet}(\mathcal{P}$, Lie $H)$. By extension, in the coupled case $L$ must depend on tensorial quantities and otherwise factors through the curvature and covariant derivative maps,

$$
L(\phi)=\tilde{L}\left(\varphi, F, D^{A} \varphi\right)=: \tilde{L}(\{\phi\}),
$$

with $\tilde{L}$ a $H$-invariant multilinear fonctional on $\Omega_{\text {tens }}^{\bullet}(\mathcal{P}): \tilde{L}(\rho(h)\{\phi\})=\tilde{L}(\{\phi\}), h \in H$ and we remind that $\rho:=(\mathrm{Ad}, \rho)$. We notice that the set $\{\phi\}=\left(F, \varphi, D^{A} \varphi\right)$ is closed under $D$, as on the one hand by Bianchi $D F=0$, and on the other hand $D^{2} \varphi=\rho_{*}(F) \varphi, D^{3} \varphi=$ $\rho_{*}(F) D \varphi$ and $D^{2 n} \varphi=\rho_{*}\left(F^{n}\right) \varphi, D^{2 n+1} \varphi=\rho_{*}\left(F^{n}\right) D \varphi$. We may denotes this $D\{\phi\} \subset\{\phi\}$. Then it comes that $\boldsymbol{d} L$ will be linear in $\boldsymbol{d} \phi$ or $D^{A}(\boldsymbol{d} \phi), \boldsymbol{d} L_{\mid \phi}=\tilde{L}\left(\boldsymbol{d} \phi, D^{A}(\boldsymbol{d} \phi) ;\{\phi\}\right)$. By (2.9)-(2.13) and using the $H$-invariance of $\tilde{L}$, we have

$$
\begin{aligned}
\boldsymbol{d} L_{\mid \phi}= & \tilde{L}\left(\boldsymbol{d} \varphi, \boldsymbol{d} F, \boldsymbol{d} D^{A}(\varphi) ;\{\phi\}\right), \\
= & \tilde{L}(\boldsymbol{d} \varphi ;\{\phi\})+\tilde{L}\left(D^{A}(\boldsymbol{d} A) ;\{\phi\}\right)+\tilde{L}\left(D^{A}(\boldsymbol{d} \varphi)+\rho_{*}(\boldsymbol{d} A) \varphi ;\{\phi\}\right), \\
= & \tilde{L}(\boldsymbol{d} \varphi ;\{\phi\})+d \tilde{L}(\boldsymbol{d} A ;\{\phi\})+\tilde{L}\left(\boldsymbol{d} A ; D^{A}\{\phi\}\right) \\
& +d \tilde{L}(\boldsymbol{d} \varphi ;\{\phi\})-\tilde{L}\left(\boldsymbol{d} \varphi ; D^{A}\{\phi\}\right)+\tilde{L}\left(\rho_{*}(\boldsymbol{d} A) \varphi ;\{\phi\}\right), \\
= & \tilde{L}\left(\boldsymbol{d} A ; D^{A}\{\phi\}\right)+\tilde{L}\left(\rho_{*}(\boldsymbol{d} A) \varphi ;\{\phi\}\right)+\tilde{L}\left(\boldsymbol{d} \varphi ;\{\phi\}-D^{A}\{\phi\}\right)+d \tilde{L}(\boldsymbol{d} \phi ;\{\phi\}), \\
= & : \tilde{E}(\boldsymbol{d} \phi ;\{\phi\})+d \tilde{\theta}(\boldsymbol{d} \phi ;\{\phi\})=E(\boldsymbol{d} \phi ; \phi)+d \theta(\boldsymbol{d} \phi ; \phi)=\boldsymbol{E}+d \boldsymbol{\theta} .
\end{aligned}
$$

The last three equalities will help keep track of what the notations of the functionals $E$ and $\theta$ means here. It is clear in particular that by (2.6), since $\{\phi\} \in \Omega_{\text {tens }}^{\bullet}(\mathcal{P})$, and due to the $H$-invariance of $\tilde{L}$, the $\mathcal{H}$-equivariance of $\boldsymbol{E}$ and $\boldsymbol{\theta}$ are trivial

$$
\begin{aligned}
& R_{\gamma}^{\star} \boldsymbol{E}=\boldsymbol{E} \quad \text { and } \quad R_{\gamma}^{\star} \boldsymbol{\theta}=\boldsymbol{\theta}, \quad \text { i.e. } \quad \boldsymbol{E}, \boldsymbol{\theta} \in \Omega_{\text {inv }}^{1}(\Phi) . \\
& \hookrightarrow \quad E\left(\boldsymbol{d} \phi^{\gamma} ; \phi^{\gamma}\right)=E(\boldsymbol{d} \phi ; \phi) \text { and } \theta\left(\boldsymbol{d} \phi^{\gamma} ; \phi^{\gamma}\right)=\theta(\boldsymbol{d} \phi ; \phi) .
\end{aligned}
$$

We can already say that their $\mathcal{H}$-gauge transformations are thus controlled only by their respective verticality properties. The latter are also related to the definition of conserved currents and charges associated with the action of $\mathcal{H}$. 
Noether currents and charges. As $L$ is basic, the infinitesimal version of its trivial equivariance is, for $\chi^{v} \in \Gamma(V \Phi), \boldsymbol{L}_{\chi^{v}} L=\iota_{\chi^{v}} \boldsymbol{d} L=\iota_{\chi^{v}} \boldsymbol{E}+d \iota_{\chi^{v}} \boldsymbol{\theta}=0$. The quantity $J(\chi ; \phi):=\iota_{\chi^{v}} \boldsymbol{\theta}$ is thus conserved on-shell, $d J(\chi ; \phi)=0_{\mid \mathcal{S}}$. This is the Noether current associated with $\mathcal{H}$. We might be interested in further determining its general form. Using the above definition (3.3) of $\boldsymbol{\theta}$ and $\boldsymbol{E}$ we have,

$$
\begin{aligned}
J(\chi ; \phi):=\iota_{\chi^{v}} \boldsymbol{\theta} & =\tilde{L}\left(\iota_{\chi^{v}} \boldsymbol{d} \phi ;\{\phi\}\right), \\
& =\tilde{L}\left(D^{A} \chi ;\{\phi\}\right)+\tilde{L}\left(-\rho_{*}(\chi) \varphi ;\{\phi\}\right), \\
& =d \tilde{L}(\chi ;\{\phi\})-\tilde{L}\left(\chi ; D^{A}\{\phi\}\right)-\tilde{L}\left(\rho_{*}(\chi) \varphi ;\{\phi\}\right), \\
& =d \theta(\chi ; \phi)-E(\chi ; \phi) .
\end{aligned}
$$

The on-shell $d$-exacteness of $J(\chi ; \phi)$ is manifest in this form. In the last equality the notation means that the current is (of course) written in terms of the Lie $H$-linear pieces of $\boldsymbol{\theta}$ and $\boldsymbol{E}$. Notice it implies that the details of the contribution of the matter field to the presymplectic potential is irrelevant to the on-shell form of the current!

The Noether charge is defined as $Q_{\Sigma}(\chi ; \phi):=\int_{\Sigma} J(\chi ; \phi)$, and is also written $Q_{\Sigma}(\chi ; \phi)=$ $\iota_{\chi} \boldsymbol{\theta}_{\Sigma}$. Given (3.5), it is explicitly

$$
Q_{\Sigma}(\chi ; \phi)=\int_{\partial \Sigma} \theta(\chi ; \phi)-\int_{\Sigma} E(\chi ; \phi) .
$$

On-shell, it is a purely boundary term. To reiterate the previous point: the above result proves that the contribution of the matter field is irrelevant to the on-shell form of the charge, which depends only on the contribution of the connection/gauge potential. Now, the presymplectic 2-form $\boldsymbol{\Theta}=\boldsymbol{d} \boldsymbol{\theta}$ induces a Poisson bracket for these charges.

Poisson bracket of charges. To see this, let us first notice that the infinitesimal version of the trivial $\mathcal{H}$-equivariance of $\boldsymbol{\theta}$, eq. (3.4), gives a relation between the Noether charge and the presymplectic 2-form

$$
\begin{aligned}
& \boldsymbol{L}_{\chi^{v}} \boldsymbol{\theta}=\iota_{\chi^{v}} \boldsymbol{d} \boldsymbol{\theta}+\boldsymbol{d} \iota_{\chi^{v}} \boldsymbol{\theta}=: \iota_{\chi^{v}} \boldsymbol{\Theta}+\boldsymbol{d} J(\chi ; \phi)=0 . \\
& \hookrightarrow \text { so that } \quad \iota_{\chi^{v}} \boldsymbol{\Theta}_{\Sigma}=-\boldsymbol{d} Q_{\Sigma}(\chi ; \phi)=-\int_{\partial \Sigma} \boldsymbol{d} \theta(\chi ; \phi)+\int_{\Sigma} \boldsymbol{d} E(\chi ; \phi) .
\end{aligned}
$$

From this, using $\left[\boldsymbol{L}_{\boldsymbol{Y}}, \iota_{\boldsymbol{X}}\right]={ }_{[\boldsymbol{Y}, \boldsymbol{X}]}$, we obtain that for $\chi^{v}, \eta^{v} \in \Gamma(V \mathcal{A})$

$$
\boldsymbol{\Theta}\left(\chi^{v}, \eta^{v}\right)=\iota_{\eta^{v}}\left(\iota_{\chi^{v}} \boldsymbol{\Theta}\right)=-\iota_{\eta^{v}} \boldsymbol{d} \iota_{\chi^{v}} \boldsymbol{\theta}=-\boldsymbol{L}_{\eta^{v}} \iota_{\chi^{v}} \boldsymbol{\theta}=-\iota_{\chi^{v}} \boldsymbol{L}_{\eta^{v}} \boldsymbol{\theta}-\iota_{\left[\eta^{v}, \chi^{v}\right]} \boldsymbol{\theta}=\iota_{[\chi, \eta]^{v}} \boldsymbol{\theta}
$$

where in the last step we use the fact that the map Lie $\mathcal{H} \rightarrow \Gamma(V \mathcal{A})$ is a isomorphism. The Poisson bracket of charges defined by the presymplectic 2 -form is thus,

$$
\left\{Q_{\Sigma}(\chi ; \phi), Q_{\Sigma}(\eta ; \phi)\right\}:=\boldsymbol{\Theta}_{\Sigma}\left(\chi^{v}, \eta^{v}\right)=\int_{\Sigma} \iota_{[\chi, \eta]^{v}} \boldsymbol{\theta}=\int_{\Sigma} J([\chi . \eta] ; \phi)=Q_{\Sigma}([\chi, \eta] ; \phi) .
$$

It is clearly antisymmetric, and the Jacobi identity is satisfied for the Poisson bracket because it holds in Lie $\mathcal{H}$. So the map Lie $\mathcal{H} \rightarrow\left(Q_{\Sigma}(; \phi),\{\},\right)$ is a Lie algebra morphism. Written functionally, (3.9) reproduces the Peierls-DeWitt bracket (see [64] Theorem 4, also [60]). 
Through this Poisson bracket, the Noether charges are also generators of Lie $\mathcal{H}$ transformations. Consider indeed a functional $f: \Phi \rightarrow \Omega^{n-1}(\mathcal{P}), \phi \mapsto f(\phi)$. Define its associated variational Hamiltonian vector field $V^{f}$, as one does, via $\iota_{V^{f}} \boldsymbol{\Theta}_{\Sigma}=-\int_{\Sigma} \boldsymbol{d} f$. The action of Lie $\mathcal{H}$ on $f$ is usually given by the Lie derivative along a vertical vector field,

$$
\begin{aligned}
\int_{\Sigma} \boldsymbol{L}_{\chi^{v}} f=\int_{\Sigma} \iota_{\chi^{v}} \boldsymbol{d} f+\boldsymbol{d}_{\iota^{\circ}} f=\iota_{\chi^{v}}\left(-\iota_{V^{f}} \boldsymbol{\Theta}_{\Sigma}\right)=\iota_{V^{f} \iota_{\chi^{v}}} \boldsymbol{\Theta}_{\Sigma}=:\left\{Q_{\Sigma}(\chi ; \phi), f\right\}, \\
\hookrightarrow=-\iota_{V^{f}} \boldsymbol{d} Q_{\Sigma}(\chi ; \phi) .
\end{aligned}
$$

The first line shows why Noether charges generate Lie $\mathcal{H}$-transformations via the Poisson bracket, the second line gives the explicit mean of computation: one must first determine the Hamiltonian vector field of $f$ via the symplectic 2 -form $\boldsymbol{\Theta}_{\Sigma}$, then feed it to the variational 1-form $\boldsymbol{d} Q_{\Sigma}(\phi ; \chi)$.

Field-dependent gauge transformations. We are interested in finding the general form of the field-dependent $\mathcal{H}$-gauge transformations of $\boldsymbol{\theta}$ and $\boldsymbol{\Theta}$. But let us first, as a warm-up, show that an invariant theory is well-behaved by finding the $\mathcal{H}$-transformation of the field equations $\boldsymbol{E}$.

As already pointed out in section 2.1, given eq. (2.4) the $\mathcal{H}$-transformation of a form depends on its $\mathcal{H}$-equivariance and its verticality property. For the field equations we have $R_{\gamma}^{\star} \boldsymbol{E}=\boldsymbol{E}$ by $(3.4)$, and $\iota_{\chi^{v}} \boldsymbol{E}=d E(\chi ; \phi)$ by $\boldsymbol{L}_{\chi^{v}} L=0$ and (3.5) above. So, for $\gamma \in \mathcal{H}$ corresponding to $\boldsymbol{\Psi} \in \operatorname{Aut}_{v}(\mathcal{A})$, we get

$$
\begin{aligned}
\boldsymbol{E}_{\mid \phi}^{\gamma}\left(\boldsymbol{X}_{\phi}\right) & :=\left(\boldsymbol{\Psi}^{\star} \boldsymbol{E}\right)_{\mid \phi}\left(\boldsymbol{X}_{\phi}\right)=\boldsymbol{E}_{\phi^{\gamma}}\left(\boldsymbol{\Psi}_{\star} \boldsymbol{X}_{\phi}\right) \\
& =\boldsymbol{E}_{\mid \phi^{\gamma}}\left(R_{\gamma(\phi) \star}\left(\boldsymbol{X}_{\phi}+\left\{\boldsymbol{d} \boldsymbol{\gamma} \gamma_{\mid \phi}^{-1}\left(\boldsymbol{X}_{\phi}\right)\right\}_{\phi}^{v}\right)\right), \\
& =R_{\gamma(\phi)}^{\star} \boldsymbol{E}_{\mid A^{\gamma}}\left(\boldsymbol{X}_{\phi}+\left\{\boldsymbol{d} \boldsymbol{\gamma} \gamma_{\mid \phi}^{-1}\left(\boldsymbol{X}_{\phi}\right)\right\}_{\phi}^{v}\right) \\
& =\boldsymbol{E}_{\mid \phi}\left(\boldsymbol{X}_{\phi}+\left\{\boldsymbol{d} \boldsymbol{\gamma} \gamma_{\mid v}^{-1}\left(\boldsymbol{X}_{\phi}\right)\right\}_{\phi}^{v}\right) \\
& =\boldsymbol{E}_{\mid \phi}\left(\boldsymbol{X}_{\phi}\right)+d E\left(\left\{\boldsymbol{d} \boldsymbol{\gamma} \gamma_{\mid \phi}^{-1}\left(\boldsymbol{X}_{A}\right)\right\} ; \phi\right),
\end{aligned}
$$

that is $\quad \boldsymbol{E}^{\gamma}=\boldsymbol{E}+d E\left(\boldsymbol{d} \gamma \boldsymbol{\gamma}^{-1} ; \phi\right)$.

The action of $\mathcal{H}$ does not take us off-shell. Which is to be expected if the bundle $\mathcal{S} \stackrel{\mathcal{H}}{\rightarrow} \mathcal{M}_{\mathcal{S}}$, and the reduced phase space $\mathcal{M}_{\mathcal{S}}$, are to be well-defined. ${ }^{18}$

The $\mathcal{H}$-transformation of $\boldsymbol{\theta}$ goes similarly. We have $R_{\gamma}^{\star} \boldsymbol{\theta}=\boldsymbol{\theta}$ by (3.4), and its verticality property is the very definition of the Noether current (3.5). Thus,

$$
\begin{aligned}
\boldsymbol{\theta}_{\mid \phi}^{\gamma}\left(\boldsymbol{X}_{\phi}\right) & :=\left(\boldsymbol{\Psi}^{\star} \boldsymbol{\theta}\right)_{\mid A}\left(\boldsymbol{X}_{\phi}\right)=\boldsymbol{\theta}_{A^{\gamma}}\left(\boldsymbol{\Psi}_{\star} \boldsymbol{X}_{\phi}\right) \\
& =\boldsymbol{\theta}_{\mid \phi^{\gamma}}\left(R_{\gamma(\phi) \star}\left(\boldsymbol{X}_{\phi}+\left\{\boldsymbol{d} \boldsymbol{\gamma} \gamma_{\mid \phi}^{-1}\left(\boldsymbol{X}_{A}\right)\right\}_{\phi}^{v}\right)\right), \\
& =R_{\gamma(\phi)}^{\star} \boldsymbol{\theta}_{\mid \phi^{\gamma}}\left(\boldsymbol{X}_{\phi}+\left\{\boldsymbol{d} \boldsymbol{\gamma} \boldsymbol{\gamma}_{\mid \phi}^{-1}\left(\boldsymbol{X}_{\phi}\right)\right\}_{\phi}^{v}\right)
\end{aligned}
$$

\footnotetext{
${ }^{18}$ But this is by no means automatic, as some non-invariant theories are not well behaved in this way (e.g. massive Yang-Mills theory, see appendix F in [33]).
} 


$$
\begin{aligned}
& =\boldsymbol{\theta}_{\mid \phi}\left(\boldsymbol{X}_{\phi}+\left\{\boldsymbol{d} \boldsymbol{\gamma} \boldsymbol{\gamma}_{\mid \phi}^{-1}\left(\boldsymbol{X}_{\phi}\right)\right\}_{\phi}^{v}\right), \\
& =\boldsymbol{\theta}_{\mid \phi}\left(\boldsymbol{X}_{\phi}\right)+J\left(\left\{\boldsymbol{d} \boldsymbol{\gamma} \boldsymbol{\gamma}_{\mid \phi}^{-1}\left(\boldsymbol{X}_{\phi}\right)\right\} ; \phi\right),
\end{aligned}
$$$$
\text { that is } \boldsymbol{\theta}^{\gamma}=\boldsymbol{\theta}+d \theta\left(\boldsymbol{d} \boldsymbol{\gamma} \boldsymbol{\gamma}^{-1} ; \phi\right)-E\left(\boldsymbol{d} \boldsymbol{\gamma} \boldsymbol{\gamma}^{-1} ; \phi\right) \text {. }
$$

The $\mathcal{H}$-transformation of the presymplectic potential is then obviously,

$$
\boldsymbol{\theta}_{\Sigma}^{\gamma}=\boldsymbol{\theta}_{\Sigma}+Q_{\Sigma}\left(\boldsymbol{d} \boldsymbol{\gamma} \boldsymbol{\gamma}^{-1} ; \phi\right)=\boldsymbol{\theta}_{\Sigma}+\int_{\partial \Sigma} \theta\left(\boldsymbol{d} \boldsymbol{\gamma} \boldsymbol{\gamma}^{-1} ; \phi\right)-\int_{\Sigma} E\left(\boldsymbol{d} \boldsymbol{\gamma} \boldsymbol{\gamma}^{-1} ; \phi\right)
$$

From this, or from (3.4)-(3.5), it is clear that the presymplectic potential is $\mathcal{H}$-invariant, basic, if we are on-shell and if either $\partial \Sigma=\emptyset$ or $\phi \rightarrow 0$ and/or $\gamma \rightarrow 1$ at $\partial \Sigma$ or at infinity.

We finally turn our attention to the $\mathcal{H}$-gauge transformation of $\boldsymbol{\Theta}:=\boldsymbol{d} \boldsymbol{\theta}$. It can be guessed from (3.12) above using the naturality of pullbacks, i.e. $\left[\boldsymbol{\Psi}^{\star}, \boldsymbol{d}\right]=0$ here. But we might want an explicit check using the method above. For this, as the verticality of $\boldsymbol{\Theta}$ is given by (3.7)-(3.8) in therm of the Noether current, we only need to determine its $\mathcal{H}$-equivariance. But then again, we must appeal to the naturality of pullbacks, in this case $\left[R_{\gamma}^{\star}, \boldsymbol{d}\right]=0$, so that $R_{\gamma}^{\star} \boldsymbol{\Theta}=R_{\gamma}^{\star} \boldsymbol{d} \boldsymbol{\theta}=\boldsymbol{d} R_{\gamma}^{\star} \boldsymbol{\theta}=\boldsymbol{d} \boldsymbol{\theta}=\boldsymbol{\Theta}$. Then,

$$
\begin{aligned}
& \boldsymbol{\Theta}^{\gamma}{ }_{\phi}\left(\boldsymbol{X}_{\phi}, \boldsymbol{Y}_{\phi}\right):=\left(\boldsymbol{\Psi}^{\star} \boldsymbol{\Theta}\right)_{\mid \phi}\left(\boldsymbol{X}_{\phi}, \boldsymbol{Y}_{\phi}\right)=\boldsymbol{\Theta}_{\mid \phi^{\gamma}}\left(\boldsymbol{\Psi}_{\star} \boldsymbol{X}_{\phi}, \boldsymbol{\Psi}_{\star} \boldsymbol{Y}_{\phi}\right), \\
& =\boldsymbol{\Theta}_{\mid \phi^{\gamma}}\left(R_{\gamma(\phi) \star}\left(\boldsymbol{X}_{\phi}+\left\{\boldsymbol{d} \boldsymbol{\gamma} \gamma_{\mid \phi}^{-1}\left(\boldsymbol{X}_{A}\right)\right\}_{\phi}^{v}\right),\right. \\
& \left.R_{\gamma(\phi) \star}\left(\boldsymbol{Y}_{\phi}+\left\{\boldsymbol{d} \gamma \boldsymbol{\gamma}_{\mid \phi}^{-1}\left(\boldsymbol{Y}_{\phi}\right)\right\}_{\phi}^{v}\right)\right) \\
& =R_{\gamma(\phi)}^{\star} \boldsymbol{\Theta}_{\mid \phi^{\gamma}}\left(\boldsymbol{X}_{\phi}+\left\{\boldsymbol{d} \boldsymbol{\gamma} \boldsymbol{\gamma}_{\mid \phi}^{-1}\left(\boldsymbol{X}_{\phi}\right)\right\}_{\phi}^{v}, \boldsymbol{Y}_{\phi}+\left\{\boldsymbol{d} \boldsymbol{\gamma} \boldsymbol{\gamma}_{\mid \phi}^{-1}\left(\boldsymbol{Y}_{\phi}\right)\right\}_{\phi}^{v}\right), \\
& =\boldsymbol{\Theta}_{\mid \phi}\left(\boldsymbol{X}_{\phi}, \boldsymbol{Y}_{\phi}\right)+\boldsymbol{\Theta}_{\mid \phi}\left(\left\{\boldsymbol{d} \boldsymbol{\gamma} \gamma_{\mid \phi}^{-1}\left(\boldsymbol{X}_{\phi}\right)\right\}_{\phi}^{v}, \boldsymbol{Y}_{\phi}\right) \\
& +\boldsymbol{\Theta}_{\mid \phi}\left(\boldsymbol{X}_{\phi},\left\{\boldsymbol{d} \gamma \gamma^{-1}{ }_{\mid \phi}\left(\boldsymbol{Y}_{\phi}\right)\right\}_{\phi}^{v}\right) \\
& +\boldsymbol{\Theta}_{\mid \phi}\left(\left\{\boldsymbol{d} \boldsymbol{\gamma} \boldsymbol{\gamma}_{\mid \phi}^{-1}\left(\boldsymbol{X}_{\phi}\right)\right\}_{\phi}^{v},\left\{\boldsymbol{d} \boldsymbol{\gamma} \boldsymbol{\gamma}_{\mid \phi}^{-1}\left(\boldsymbol{Y}_{\phi}\right)\right\}_{\phi}^{v}\right), \\
& =\boldsymbol{\Theta}_{\mid \phi}\left(\boldsymbol{X}_{v}, \boldsymbol{Y}_{\phi}\right)-\iota_{\boldsymbol{Y}} \boldsymbol{d} J\left(\left\{\boldsymbol{d} \boldsymbol{\gamma} \boldsymbol{\gamma}_{\mid \phi}^{-1}\left(\boldsymbol{X}_{\phi}\right)\right\} ; \phi\right)+\iota_{\boldsymbol{X}} \boldsymbol{d} J\left(\left\{\boldsymbol{d} \boldsymbol{\gamma} \boldsymbol{\gamma}_{\mid \phi}^{-1}\left(\boldsymbol{Y}_{\phi}\right)\right\} ; \phi\right) \\
& +\boldsymbol{\theta}_{\mid \phi}\left(\left[\boldsymbol{d} \boldsymbol{\gamma} \boldsymbol{\gamma}_{\mid \phi}^{-1}\left(\boldsymbol{X}_{\phi}\right), \boldsymbol{d} \boldsymbol{\gamma} \boldsymbol{\gamma}_{\mid \phi}^{-1}\left(\boldsymbol{Y}_{A}\right)\right]_{\phi}^{v}\right) \\
& =\boldsymbol{\Theta}_{\mid \phi}\left(\boldsymbol{X}_{\phi}, \boldsymbol{Y}_{\phi}\right)-\boldsymbol{Y} \cdot J\left(\left\{\boldsymbol{d} \boldsymbol{\gamma} \gamma_{\mid \phi}^{-1}\left(\boldsymbol{X}_{\phi}\right)\right\} ; A\right)+\boldsymbol{X} \cdot J\left(\left\{\boldsymbol{d} \boldsymbol{\gamma} \boldsymbol{\gamma}_{\mid \phi}^{-1}\left(\boldsymbol{Y}_{\phi}\right)\right\} ; A\right) \\
& +J\left(\left\{\left[\boldsymbol{d} \boldsymbol{\gamma} \boldsymbol{\gamma}_{\mid \phi}^{-1}\left(\boldsymbol{X}_{\phi}\right), \boldsymbol{d} \boldsymbol{\gamma} \boldsymbol{\gamma}_{\mid \phi}^{-1}\left(\boldsymbol{Y}_{\phi}\right)\right]\right\} ; \phi\right) \text {. }
\end{aligned}
$$

Notice that in the step before last, we used (3.8), and (3.7) which is only valid for $\phi$ independent gauge parameters $\chi \in$ Lie $\mathcal{H}$. So the quantity $\boldsymbol{d} \boldsymbol{\gamma} \gamma_{\mid \phi}^{-1}\left(\boldsymbol{Z}_{\phi}\right)$ is considered $\phi$ independent, and only the underlined $\phi$ 's in $J\left(\left\{\boldsymbol{d} \boldsymbol{\gamma} \boldsymbol{\gamma}_{\mid \phi}^{-1}\left(\boldsymbol{Z}_{\phi}\right)\right\} ; \underline{\phi}\right)$ are acted upon by the variational vector fields in (3.14). 
Actually, the quantity $\boldsymbol{d} J\left(\left\{\boldsymbol{d} \gamma \gamma_{\mid \phi}^{-1}\right\} ; \phi\right)$ is a 2-form on $\Phi$, and by the Koszul formula, evaluated on two vectors it gives

$$
\begin{aligned}
\boldsymbol{d} J\left(\left\{\boldsymbol{d} \boldsymbol{\gamma} \boldsymbol{\gamma}_{\mid \phi}^{-1}\right\} ; \phi\right)\left(\boldsymbol{X}_{\phi}, \boldsymbol{Y}_{\phi}\right)= & \boldsymbol{X} \cdot J\left(\left\{\boldsymbol{d} \boldsymbol{\gamma} \gamma_{\mid \phi}^{-1}\left(\boldsymbol{Y}_{\phi}\right)\right\} ; \phi\right)-\boldsymbol{Y} \cdot J\left(\left\{\boldsymbol{d} \boldsymbol{\gamma} \boldsymbol{\gamma}_{\mid \phi}^{-1}\left(\boldsymbol{X}_{\phi}\right)\right\} ; \phi\right) \\
& -J\left(\left\{\boldsymbol{d} \boldsymbol{\gamma} \gamma_{\mid \phi}^{-1}\left([\boldsymbol{X}, \boldsymbol{Y}]_{\phi}\right)\right\} ; \phi\right)
\end{aligned}
$$

where all the $\phi$ 's in the terms $J\left(\left\{\boldsymbol{d} \gamma \gamma_{\mid \phi}^{-1}\left(\boldsymbol{Z}_{\phi}\right)\right\} ; \phi\right)$ are acted upon. Observe also that

$$
\begin{aligned}
{\left[\boldsymbol{d} \gamma \boldsymbol{\gamma}^{-1}(\boldsymbol{X}), \boldsymbol{d} \boldsymbol{\gamma} \boldsymbol{\gamma}^{-1}(\boldsymbol{Y})\right] } & =\boldsymbol{d} \boldsymbol{\gamma} \boldsymbol{\gamma}^{-1}(\boldsymbol{X}) \boldsymbol{d} \boldsymbol{\gamma} \boldsymbol{\gamma}^{-1}(\boldsymbol{Y})-\boldsymbol{d} \boldsymbol{\gamma} \boldsymbol{\gamma}^{-1}(\boldsymbol{Y}) \boldsymbol{d} \boldsymbol{\gamma} \boldsymbol{\gamma}^{-1}(\boldsymbol{X}) \\
& =-\boldsymbol{d} \boldsymbol{\gamma}(\boldsymbol{X}) \boldsymbol{d} \gamma^{-1}(\boldsymbol{Y})+\boldsymbol{d} \gamma(\boldsymbol{Y}) \boldsymbol{d} \gamma^{-1}(\boldsymbol{X}) \\
& =\left(-\boldsymbol{d} \boldsymbol{d} \boldsymbol{d} \gamma^{-1}\right)(\boldsymbol{X}, \boldsymbol{Y})=\boldsymbol{d}\left(\boldsymbol{d} \boldsymbol{\gamma} \gamma^{-1}\right)(\boldsymbol{X}, \boldsymbol{Y})
\end{aligned}
$$

which is simply a "flatness", or Maurer-Cartan type, condition $\boldsymbol{d}\left(\boldsymbol{d} \boldsymbol{\gamma} \boldsymbol{\gamma}^{-1}\right)$ $\frac{1}{2}\left[\boldsymbol{d} \boldsymbol{\gamma} \boldsymbol{\gamma}^{-1}, \boldsymbol{d} \boldsymbol{\gamma} \boldsymbol{\gamma}^{-1}\right]=0$. But then, again by Koszul we have,

$$
\begin{aligned}
{\left[\boldsymbol{d} \boldsymbol{\gamma} \boldsymbol{\gamma}_{\mid \phi}^{-1}\left(\boldsymbol{X}_{\phi}\right), \boldsymbol{d} \boldsymbol{\gamma} \boldsymbol{\gamma}_{\mid \phi}^{-1}\left(\boldsymbol{Y}_{\phi}\right)\right] } & =\boldsymbol{d}\left(\boldsymbol{d} \boldsymbol{\gamma} \boldsymbol{\gamma}^{-1}\right)_{\mid \phi}\left(\boldsymbol{X}_{\phi}, \boldsymbol{Y}_{\phi}\right) \\
& =\boldsymbol{X} \cdot\left\{\boldsymbol{d} \boldsymbol{\gamma} \boldsymbol{\gamma}_{\mid \underline{\phi}}^{-1}\left(\boldsymbol{Y}_{\underline{\phi}}\right)\right\}-\boldsymbol{Y} \cdot\left\{\boldsymbol{d} \boldsymbol{\gamma} \boldsymbol{\gamma}_{\mid \underline{\phi}}^{-1}\left(\boldsymbol{X}_{\underline{\phi}}\right)\right\}-\boldsymbol{d} \boldsymbol{\gamma} \boldsymbol{\gamma}_{\phi}^{-1}\left([\boldsymbol{X}, \boldsymbol{Y}]_{\phi}\right),
\end{aligned}
$$

where we stressed that the underlined $\phi$ 's are acted upon. Inserting this in the last term of (3.14), remembering that $J(; \phi)$ is linear in the first argument and using (3.15), we have

$$
\begin{aligned}
\Theta_{\mid \phi}^{\gamma}\left(\boldsymbol{X}_{\phi}, \boldsymbol{Y}_{\phi}\right)= & \boldsymbol{\Theta}_{\mid \phi}\left(\boldsymbol{X}_{\phi}, \boldsymbol{Y}_{\phi}\right)+\boldsymbol{X} \cdot J\left(\left\{\boldsymbol{d} \boldsymbol{\gamma} \gamma_{\mid \phi}^{-1}\left(\boldsymbol{Y}_{\phi}\right)\right\} ; \underline{\phi}\right)-\boldsymbol{Y} \cdot J\left(\left\{\boldsymbol{d} \boldsymbol{\gamma} \boldsymbol{\gamma}_{\mid \phi}^{-1}\left(\boldsymbol{X}_{\phi}\right)\right\} ; \underline{\phi}\right) \\
& +J\left(\left\{\boldsymbol{X} \cdot\left\{\boldsymbol{d} \boldsymbol{\gamma} \gamma_{\mid \underline{\phi}}^{-1}\left(\boldsymbol{Y}_{\underline{\phi}}\right)\right\}-\boldsymbol{Y} \cdot\left\{\boldsymbol{d} \boldsymbol{\gamma} \gamma_{\mid \underline{\phi}}^{-1}\left(\boldsymbol{X}_{\underline{\phi}}\right)\right\}-\boldsymbol{d} \boldsymbol{\gamma} \boldsymbol{\gamma}_{\phi}^{-1}\left([\boldsymbol{X}, \boldsymbol{Y}]_{\phi}\right)\right\} ; \phi\right), \\
= & \boldsymbol{\Theta}_{\mid \phi}\left(\boldsymbol{X}_{\phi}, \boldsymbol{Y}_{\phi}\right)+\boldsymbol{X} \cdot J\left(\left\{\boldsymbol{d} \boldsymbol{\gamma} \boldsymbol{\gamma}_{\mid \underline{\phi}}^{-1}\left(\boldsymbol{Y}_{\underline{\phi}}\right)\right\} ; \underline{\phi}\right) \\
& -\boldsymbol{Y} \cdot J\left(\left\{\boldsymbol{d} \boldsymbol{\gamma} \gamma_{\mid \underline{\phi}}^{-1}\left(\boldsymbol{X}_{\underline{\phi}}\right)\right\} ; \underline{\phi}\right)-J\left(\left\{\boldsymbol{d} \boldsymbol{\gamma} \gamma_{\mid \phi}^{-1}\left([\boldsymbol{X}, \boldsymbol{Y}]_{\phi}\right)\right\} ; \phi\right), \\
= & \boldsymbol{\Theta}_{\mid \phi}\left(\boldsymbol{X}_{\phi}, \boldsymbol{Y}_{\phi}\right)+\boldsymbol{d} J\left(\left\{\boldsymbol{d} \boldsymbol{\gamma} \gamma_{\mid \phi}^{-1}\right\} ; \phi\right)\left(\boldsymbol{X}_{\phi}, \boldsymbol{Y}_{\phi}\right) .
\end{aligned}
$$

Which is finally, using (3.5),

$$
\boldsymbol{\Theta}^{\gamma}=\boldsymbol{\Theta}+\boldsymbol{d} J\left(\left\{\boldsymbol{d} \boldsymbol{\gamma} \boldsymbol{\gamma}^{-1}\right\} ; \phi\right)=\boldsymbol{\Theta}+\boldsymbol{d}\left(d \theta\left(\boldsymbol{d} \gamma \boldsymbol{\gamma}^{-1} ; \phi\right)-E\left(\boldsymbol{d} \boldsymbol{\gamma} \boldsymbol{\gamma}^{-1} ; \phi\right)\right),
$$

consistent with (3.12). The $\mathcal{H}$-gauge transformation of the presymplectic 2 -form is then,

$$
\Theta_{\Sigma}^{\gamma}=\Theta_{\Sigma}+d Q_{\Sigma}\left(\boldsymbol{d} \gamma \gamma^{-1} ; \phi\right)=\Theta_{\Sigma}+\int_{\partial \Sigma} \boldsymbol{d} \theta\left(\boldsymbol{d} \gamma \gamma^{-1} ; \phi\right)-\int_{\Sigma} \boldsymbol{d} E\left(\boldsymbol{d} \gamma \gamma^{-1} ; \phi\right)
$$

consistent with (3.13). As for $\boldsymbol{\theta}_{\Sigma}$, the presymplectic 2 -form is $\mathcal{H}$-invariant, basic, if we are on-shell and if either $\partial \Sigma=\emptyset$ or $\phi \rightarrow 0$ and/or $\gamma \rightarrow 1$ at $\partial \Sigma$ or at infinity. In which case it induces a symplectic 2 -form on $\mathcal{M}_{\mathcal{S}}$.

We can use the results just derived to say a word about the charges associated with field-dependent gauge parameters $\chi \in$ Lie $\mathcal{H}$ and their Poisson bracket. 
On charges and bracket associated with field-dependent gauge parameters. As $L \in \Omega_{\text {inv }}^{0}(\Phi)$, we have $L^{\gamma}=L$ for $\gamma \in \mathcal{H}$, whose linearisation is $\boldsymbol{L}_{\chi^{v}} L=0$ for $\boldsymbol{\chi} \in$ Lie $\mathcal{H}$. As the latter relation is $\iota_{\chi^{v}} \boldsymbol{E}+d \iota_{\chi^{v}} \boldsymbol{\theta}=0$, it is still true that the current associated with a field-dependent gauge parameter defined by

$$
J(\chi ; \phi):=\iota^{v} \boldsymbol{\theta}=d \theta(\boldsymbol{\chi} ; \phi)-E(\boldsymbol{\chi} ; \phi)
$$

is conserved on-shell. Integration over a codimension 1 submanifold $\Sigma$ defines the associated Noether charge,

$$
Q_{\Sigma}(\chi ; \phi)=\int_{\partial \Sigma} \theta(\chi ; \phi)-\int_{\Sigma} E(\chi ; \phi)
$$

The question is now to find the relation of this current/charge to the presymplectic potential and 2-form.

This will be easily done after we have clarified a few technical points. First, let us define the bracket

$$
\{\boldsymbol{\chi}, \boldsymbol{\eta}\}:=[\boldsymbol{\chi}, \boldsymbol{\eta}]+\boldsymbol{\chi}^{v}(\boldsymbol{\eta})-\boldsymbol{\eta}^{v}(\boldsymbol{\chi}),
$$

where the first term on the right-hand side is the standard Lie bracket in Lie $H$. Of course, for $\boldsymbol{\chi}, \boldsymbol{\eta} \in$ Lie $\mathcal{H}$ we have that $\boldsymbol{\chi}^{v}(\boldsymbol{\eta})=[\boldsymbol{\eta}, \boldsymbol{\chi}],{ }^{19}$ so (3.20) reduces to $\{\boldsymbol{\chi}, \boldsymbol{\eta}\}=-[\boldsymbol{\chi}, \boldsymbol{\eta}]$. While for $\chi, \eta \in$ Lie $\mathcal{H}$ this bracket reduces to the standard one $\{\chi, \eta\}=[\chi, \eta]$. We will keep the general bracket because we have in mind applications of this formalism where the relevant transformation group is not a gauge group $\mathcal{H}$, but $\operatorname{Diff}(M)$. In this case, the linearised gauge parameters are vector fields $\chi, \eta \in \operatorname{LieDiff}(M) \simeq \Gamma(T M)$, with [,] the Lie bracket of vector fields. Then, $\boldsymbol{\chi}, \boldsymbol{\eta}: \Phi \rightarrow \Gamma(T M)$ are field-dependent vector fields s.t. $\chi^{v}(\boldsymbol{\eta}) \neq[\boldsymbol{\eta}, \boldsymbol{\chi}]$ and is generally left unspecified.

The bracket (3.20) enters the following commutation relations, proven in appendix C,

$$
\begin{aligned}
{\left[\iota_{\chi^{v}}, \iota_{\boldsymbol{d}} \boldsymbol{\eta}^{v}\right] } & =\iota_{\left[\boldsymbol{\chi}^{v}(\boldsymbol{\eta})\right]^{v}}, \\
{\left[\boldsymbol{L}_{\boldsymbol{\chi}^{v}}, \iota_{\boldsymbol{\eta}^{v}}\right] } & =\iota_{\{\boldsymbol{\chi}, \boldsymbol{\eta}\}^{v}}, \\
{\left[\boldsymbol{L}_{\boldsymbol{\chi}^{v}}, \boldsymbol{L}_{\boldsymbol{\eta}^{v}}\right] } & =\boldsymbol{L}_{\{\boldsymbol{\chi}, \boldsymbol{\eta}\}^{v}} .
\end{aligned}
$$

The third is a consequence of the second. Notice that in the case $\chi, \eta \in$ Lie $\mathcal{H}$ we recover $\left[\boldsymbol{L}_{\chi^{v}}, \boldsymbol{L}_{\eta^{v}}\right]=\boldsymbol{L}_{\left[\chi^{v}, \eta^{v}\right]}$, since $[\chi, \eta]^{v}=\left[\chi^{v}, \eta^{v}\right]$. While in the case $\boldsymbol{\chi}, \boldsymbol{\eta} \in$ Lie $\mathcal{H}$ we also recover $\left[\boldsymbol{L}_{\chi^{v}}, \boldsymbol{L}_{\boldsymbol{\eta}^{v}}\right]=\boldsymbol{L}_{\left[\chi^{v}, \boldsymbol{\eta}^{v}\right]}$, since $-[\boldsymbol{\chi}, \boldsymbol{\eta}]^{v}=\left[\boldsymbol{\chi}^{v}, \boldsymbol{\eta}^{v}\right]$. In line with the general formula $\left[\boldsymbol{L}_{\boldsymbol{X}}, \boldsymbol{L}_{\boldsymbol{Y}}\right]=\boldsymbol{L}_{[\boldsymbol{X}, \boldsymbol{Y}]}$ valid $\forall \boldsymbol{X}, \boldsymbol{Y} \in \Gamma(T \Phi)$.

With the above relation at hand, we proceed. The linearisation version of the $\mathcal{H}$ transformation of $\boldsymbol{\theta}$, eq. (3.12), is $\boldsymbol{L}_{\boldsymbol{\chi}^{v}} \boldsymbol{\theta}=J(\boldsymbol{d} \boldsymbol{\chi} ; \phi)=\iota_{\boldsymbol{d} \boldsymbol{\boldsymbol { \chi } ^ { v }}} \boldsymbol{\theta}$. That is,

$$
\begin{aligned}
{ }^{\iota} \chi^{v} \boldsymbol{\Theta} & =-\boldsymbol{d} J(\boldsymbol{\chi} ; \phi)+J(\boldsymbol{d} \boldsymbol{\chi} ; \phi), \\
& =-\boldsymbol{d} J(\underline{\boldsymbol{\chi}} ; \phi) .
\end{aligned}
$$

The notation in the second line is meant to suggest that, given the linearity of $J(; \phi)$ in its first argument, the first line gives the same result as eq. (3.7) for a field-independent parameter $\chi$. That is, if $\chi$ was held $(\phi-)$ constant, the result would of course be integrable. ${ }^{20}$

\footnotetext{
${ }^{19}$ Which is the linearisation of the $\mathcal{H}$-gauge transformation law $\boldsymbol{\eta}^{\gamma}=\boldsymbol{\gamma}^{-1} \boldsymbol{\eta} \boldsymbol{\gamma}$, itself resulting from the defining $\mathcal{H}$-equivariance of $\boldsymbol{\eta} \in$ Lie $\mathcal{H}, R_{\gamma}^{\star} \boldsymbol{\eta}=\gamma^{-1} \boldsymbol{\eta} \gamma$.

${ }^{20}$ This is often denoted as $\iota_{\chi^{v}} \Theta=-\not J J(\chi ; \phi)$ in the extent literature.
} 
From (3.22) follows immediately the relation between the presymplectic 2 -form and the charge for field-dependent parameters,

$$
\begin{aligned}
\iota_{\chi^{v}} \boldsymbol{\Theta}_{\Sigma} & =-\boldsymbol{d} Q_{\Sigma}(\boldsymbol{\chi} ; \phi)+Q_{\Sigma}(\boldsymbol{d} \boldsymbol{\chi} ; \phi), \\
& =-\boldsymbol{d} Q_{\Sigma}(\underline{\boldsymbol{\chi}} ; \phi) .
\end{aligned}
$$

The non-integrable term $Q_{\Sigma}(\boldsymbol{d} \boldsymbol{\chi} ; \phi)$ is sometimes called 'symplectic flux' - e.g. in [65].

In this circumstance, can we still define a Poisson Bracket for the charges (3.19)? As it turns out yes, and it has the same expression as in the $\phi$-independent case. To see this, consider

$$
\begin{aligned}
& \boldsymbol{\Theta}\left(\boldsymbol{\chi}^{v}, \boldsymbol{\eta}^{v}\right)=\iota_{\boldsymbol{\eta}^{v}}\left(\iota_{\boldsymbol{\chi}^{v}} \boldsymbol{\Theta}\right)=-\iota_{\boldsymbol{\eta}^{v}} \boldsymbol{d} \iota_{\boldsymbol{\chi}^{v}} \boldsymbol{\theta}+\iota_{\boldsymbol{\eta}^{v}} \iota_{\boldsymbol{d}} \boldsymbol{\chi}^{v} \boldsymbol{\theta} \quad \text { by (3.22), }
\end{aligned}
$$

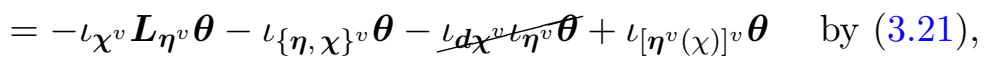

$$
\begin{aligned}
& =-\iota_{\chi^{v}}\left(\iota_{\boldsymbol{d}} \boldsymbol{\eta}^{v} \boldsymbol{\theta}\right)+\iota_{\{\chi, \boldsymbol{\eta}\}^{v}} \boldsymbol{\theta}+\iota_{\left[\boldsymbol{\eta}^{v}(\chi)\right]^{v}} \boldsymbol{\theta} \\
& =-\iota_{d \eta^{v}} \chi^{v} \boldsymbol{\theta}-\iota_{\left[\chi^{v}(\boldsymbol{\eta})\right]^{v}} \boldsymbol{\theta}+\iota_{\{\boldsymbol{\chi}, \boldsymbol{\eta}\}^{v}} \boldsymbol{\theta}+\iota_{\left[\boldsymbol{\eta}^{v}(\chi)\right]^{v}} \boldsymbol{\theta}=\iota_{[\chi, \boldsymbol{\eta}]^{v}} \boldsymbol{\theta},
\end{aligned}
$$

by definition of the extended bracket (3.20) in the last step. If we define the Poisson bracket the usual way, we get

$$
\left\{Q_{\Sigma}(\boldsymbol{\chi} ; \phi), Q_{\Sigma}(\boldsymbol{\eta} ; \phi)\right\}:=\boldsymbol{\Theta}_{\Sigma}\left(\boldsymbol{\chi}^{v}, \boldsymbol{\eta}^{v}\right)=\int_{\Sigma}{ }^{\iota}[\chi, \boldsymbol{\eta}]^{v} \boldsymbol{\theta}=: Q_{\Sigma}([\boldsymbol{\chi}, \boldsymbol{\eta}] ; \phi)
$$

by (3.18)-(3.19). This matches eq. (3.9), and obviously reduces to it when $\chi \rightarrow \chi$. The Noether charges (3.19) for $\phi$-dependent gauge parameters $Q_{\Sigma}(\boldsymbol{\chi} ; \phi)$ generate Lie $\mathcal{H}$ transformation via the PB (3.25).

In [33] a centrally extended PB of charges for $\phi$-independent parameter was defined for anomalous (non-invariant) gauge theories. We will show elsewhere that non-integrable charges for $\phi$-dependent parameters are defined similarly as above and that their PB is the same as the centrally extended one just mentioned.

In the final paragraph of this subsection, we address the question of the physical status, and observability, of the Noether charges derived in this framework.

On the observability of charges. Physically interpretable charges are computed against a background field configuration enjoying special symmetries, in asymptotic domains of spacetime where the theory can be approximated by its free regime [36, 66]. Let us see how to make contact with this viewpoint in the above framework. Using the affine structure of $\mathcal{A}$, we suppose that the connexion (the gauge potential) is written,

$$
A=A_{0}+\alpha
$$

with $A_{0}$ a background connection, s.t. by definition of the term $\boldsymbol{d} A_{0}=0$, and $\alpha \in$ $\Omega_{\text {tens }}^{1}(\mathcal{P}, \operatorname{Lie} H)$. We have then,

$$
\begin{aligned}
\boldsymbol{d} A & =\boldsymbol{d} \alpha, \\
F & =F_{0}+f+\frac{1}{2}[\alpha, \alpha], \quad \text { and } \quad \boldsymbol{d} F=D^{A}(\boldsymbol{d} \alpha),
\end{aligned}
$$


where $F_{0}$ is the background curvature $\left(\boldsymbol{d} F_{0}=0\right)$ and $f:=D^{A_{0}} \alpha=D_{0} \alpha$ is the field strength we associate to the potential $\alpha$. The field equations and presymplectic potential are then

$$
\boldsymbol{E}=E(\boldsymbol{d} \phi ; \phi)=E(\boldsymbol{d} \alpha, \boldsymbol{d} \varphi ; \phi) \quad \text { and } \quad \boldsymbol{\theta}=\theta(\boldsymbol{d} \phi ; \phi)=\theta(\boldsymbol{d} \alpha, \boldsymbol{d} \varphi ; \phi),
$$

with functionnal expressions defined in (3.3). Plugging the ansatz (3.26) in the Noether current (3.5) and using (3.3), we have on the one hand

$$
\begin{aligned}
J(\chi ; \phi)=\iota_{\chi^{v}} \boldsymbol{\theta} & =\tilde{L}\left(\iota_{\chi^{v}} \boldsymbol{d} \alpha ;\{\phi\}\right)+\tilde{L}\left(\iota_{\chi^{v}} \boldsymbol{d} \varphi ;\{\phi\}\right), \\
& =\tilde{L}([\alpha, \chi] ;\{\phi\})-\tilde{L}\left(\rho_{*}(\chi) \varphi ;\{\phi\}\right), \\
& =-\tilde{L}(\chi ;[\alpha,\{\phi\}])-\tilde{L}\left(\rho_{*}(\chi) \varphi ;\{\phi\}\right),
\end{aligned}
$$

by the $H$-invariance of $\tilde{L}$ in the last step. On the other hand the field equations are,

$$
\begin{aligned}
\boldsymbol{E}=E(\boldsymbol{d} \alpha, \boldsymbol{d} \varphi ; \phi) & =\tilde{L}\left(\boldsymbol{d} \alpha ; D^{A}\{\phi\}\right)+\tilde{L}\left(\rho_{*}(\boldsymbol{d} \alpha) \varphi ;\{\phi\}\right)+\tilde{L}\left(\boldsymbol{d} \varphi ;\{\phi\}-D^{A}\{\phi\}\right), \\
& =\tilde{L}\left(\boldsymbol{d} \alpha ; D_{0}\{\phi\}+[\alpha,\{\phi\}]\right)+\tilde{L}\left(\rho_{*}(\boldsymbol{d} \alpha) \varphi ;\{\phi\}\right)+\tilde{L}\left(\boldsymbol{d} \varphi ;\{\phi\}-D^{A}\{\phi\}\right) .
\end{aligned}
$$

Therefore, we get to write the current above in terms of the Lie $H$-linear piece of $\boldsymbol{E}$, so that:

$$
\begin{aligned}
J(\chi ; \phi) & =\tilde{L}\left(\chi ; D_{0}\{\phi\}\right)-E(\chi ;\{\phi\}), \\
& =d \tilde{L}(\chi,\{\phi\})-\tilde{L}\left(D_{0} \chi ;\{\phi\}\right)-E(\chi ;\{\phi\}), \\
& =d \theta(\chi, \phi)-\tilde{L}\left(D_{0} \chi ;\{\phi\}\right)-E(\chi ; \phi) .
\end{aligned}
$$

This result may be interpreted as meaning that the current is conserved if we are on-shell and if $\chi$ is a Killing symmetry of the background connection $A_{0}, D_{0} \chi=0$. The associated Noether charges $Q_{\Sigma}(\chi ; \phi)=Q_{\Sigma}(\chi ; \alpha, \varphi)$ can then be seen as conserved charges associated with the symmetries of the background. Notice that the field equations for the matter field, the piece of $\boldsymbol{E}$ linear in $\boldsymbol{d} \varphi$, plays no role in this.

We notice that (3.29) with the Killing condition is formally the same as eq. (3.5). This means that the general expressions for the currents and charges (3.5)-(3.6) can be used from the onset, simply plugging in the ansatz (3.26) (in concrete situations) and declaring $\chi$ to be a Killing symmetry for $A_{0}$. What one typically gets from doing so, is that $Q_{\Sigma}(\chi ; \alpha, \varphi)$ splits as a background contribution from $A_{0}$ - treated as a renormalisation constant and a physically interpreted contribution from $\alpha$, considered as the dynamical gauge field.

This in particular reproduces and generalises the treatment of Abbott and Deser [34, 35] who derive charges in classical Yang-Mills theory and in metric gravity. For comparison, we re-express in the language of differential forms their treatment of the YM case in appendix B. Finally, we remark that the above manifestly holds for $\phi$-dependent gauge parameters $\chi$ as well.

We now illustrate the general results of the previous subsections with the classic examples of Yang-Mills theory and for 4D gravity.

\subsection{Applications}

In the following applications, we will consider that all the geometric objects are pulled-back on spacetime $M$. So that a point in field space $\phi=(A, \varphi) \in \Phi$ can now be understood as referring to local fields, i.e. $A$ is a local connection - that is a gauge potential - and $\varphi \in \Gamma(E)$ is indeed a section of the associated bundle $E$. 


\subsubsection{Coupled Yang-Mills theory}

The Lagrangian describing the coupling of a Yang-Mills field to a $H=\mathrm{SU}(n)$ multiplet scalar field (that we keep massless to avoid unnecessary complications) is

$$
L_{\mathrm{YM}}(\phi)=L_{\mathrm{YM}}(A, \varphi)=\frac{1}{2} \operatorname{Tr}(F * F)+\frac{1}{2}\langle D \varphi, * D \varphi\rangle,
$$

and it is clear that $R_{\gamma}^{\star} L_{\mathrm{YM}}=L_{\mathrm{YM}}$ for $\gamma \in \mathcal{H}=\mathcal{S U}(n)$, i.e. $L_{\mathrm{YM}} \in \Omega_{\text {basic }}^{0}(\Phi)$. Thus, using $\langle u, v\rangle=\operatorname{Tr}(|v\rangle\langle u|)$,

$$
\begin{aligned}
\boldsymbol{d} L_{\mathrm{YM}} & =\boldsymbol{E}_{\mathrm{YM}}+d \boldsymbol{\theta}_{\mathrm{YM}} \quad \in \Omega_{\text {basic }}^{1}(\Phi) \quad \text { with: } \\
\boldsymbol{E}_{\mathrm{YM}} & =E_{\mathrm{YM}}(\boldsymbol{d} A ; A)=\operatorname{Tr}(\boldsymbol{d} A\{D * F-|* D \varphi\rangle\langle\varphi|\})+\langle\boldsymbol{d} \varphi, D * D \varphi\rangle \quad \in \Omega_{\mathrm{inv}}^{1}(\Phi), \\
\boldsymbol{\theta}_{\mathrm{YM}} & =\theta_{\mathrm{YM}}(\boldsymbol{d} A ; A)=\operatorname{Tr}(\boldsymbol{d} A * F)+\langle\boldsymbol{d} \varphi, * D \varphi\rangle \quad \in \Omega_{\mathrm{inv}}^{1}(\Phi) .
\end{aligned}
$$

We denote $J=|* D \varphi\rangle\langle\varphi|$ the $(n-1)$-form current sourcing the YM field. By the general formula (3.6), the Noether charge associated with $\chi \in \operatorname{Lie} \mathcal{S U}(n)$ is then

$$
\begin{aligned}
Q_{\Sigma}^{\mathrm{YM}}(\chi ; \phi) & =\int_{\partial \Sigma} \theta_{\mathrm{YM}}(\chi ; \phi)-\int_{\Sigma} E_{\mathrm{YM}}(\chi ; \phi), \\
& =\int_{\partial \Sigma} \operatorname{Tr}(\chi * F)-\int_{\Sigma} \operatorname{Tr}(\chi\{D * F-J\}) .
\end{aligned}
$$

A result that can be checked by direct computation from the definition $Q_{\Sigma}^{\mathrm{YM}}(\chi ; \phi):=$ $\iota_{\chi^{v}} \boldsymbol{\theta}_{\Sigma}^{\mathrm{YM}}$, using (3.32) and (2.5). Notice how only the Lie $H$-linear piece of $\boldsymbol{E}_{\mathrm{YM}}$ contributes. On-shell, this charge is the same as in the pure YM case, $Q_{\Sigma}^{\mathrm{YM}}(\chi ; \phi)=Q_{\Sigma}^{\mathrm{YM}}(\chi ; A){ }_{\mid \mathcal{S}}$ (see [33] section 5.1.1). The presymplectic 2 -form is,

$$
\boldsymbol{\Theta}_{\Sigma}^{\mathrm{YM}}=\int_{\Sigma} \boldsymbol{d} \boldsymbol{\theta}_{\mathrm{YM}}=-\int_{\Sigma} \operatorname{Tr}(\boldsymbol{d} A * \boldsymbol{d} F)-\langle\boldsymbol{d} \varphi, * \boldsymbol{d} D \varphi\rangle \quad \in \Omega_{\mathrm{inv}}^{2}(\Phi) .
$$

and by (3.7) relates to the charge as

$$
\iota_{\chi^{v}} \boldsymbol{\Theta}_{\Sigma}^{\mathrm{YM}}=-\boldsymbol{d} Q_{\Sigma}^{\mathrm{YM}}(\chi ; \phi)=-\int_{\partial \Sigma} \operatorname{Tr}(\chi * \boldsymbol{d} F)+\int_{\Sigma} \operatorname{Tr}(\chi \boldsymbol{d}\{D * F-J\}) .
$$

By (3.9) it induces the Poisson bracket of charges $\left\{Q_{\Sigma}^{\mathrm{YM}}(\chi ; \phi), Q_{\Sigma}^{\mathrm{YM}}(\eta ; \phi)\right\}=Q_{\Sigma}^{\mathrm{YM}}([\chi, \eta] ; \phi)$, as could be checked explicitly by computing $\boldsymbol{\Theta}_{\Sigma}^{\mathrm{YM}}\left(\chi^{v}, \eta^{v}\right)$ from (3.34) - and using (2.5), (2.10) and (2.14). The map $\chi \rightarrow Q_{\Sigma}^{\mathrm{YM}}(\chi ; \phi)$ is thus a morphism of Lie algebras.

By (3.19), the charge associated with a field-dependent gauge parameter $\chi \in$ Lie $\mathcal{H}$ is

$$
\begin{aligned}
Q_{\Sigma}^{\mathrm{YM}}(\chi ; \phi) & =\int_{\partial \Sigma} \theta_{\mathrm{YM}}(\chi ; \phi)-\int_{\Sigma} E_{\mathrm{YM}}(\chi ; \phi), \\
& =\int_{\partial \Sigma} \operatorname{Tr}(\chi * F)-\int_{\Sigma} \operatorname{Tr}(\chi\{D * F-J\}) .
\end{aligned}
$$

This time it is non-integrable, as by (3.23) we have

$$
\begin{aligned}
{ }_{\iota^{v}} \boldsymbol{\Theta}_{\Sigma}^{\mathrm{YM}} & =-\boldsymbol{d} Q_{\Sigma}^{\mathrm{YM}}(\boldsymbol{\chi} ; \phi)+Q_{\Sigma}^{\mathrm{YM}}(\boldsymbol{d} \boldsymbol{\chi} ; \phi)=-\boldsymbol{d} Q_{\Sigma}(\underline{\boldsymbol{\chi}} ; \phi), \\
& =-\int_{\partial \Sigma} \operatorname{Tr}(\boldsymbol{\chi} * \boldsymbol{d} F)+\int_{\Sigma} \operatorname{Tr}(\boldsymbol{\chi} \boldsymbol{d}\{D * F-J\}) .
\end{aligned}
$$


Still, by (3.25) the non-integrable charges (3.36) satisfy the Poisson algebra $\left\{Q_{\Sigma}^{\mathrm{YM}}(\boldsymbol{\chi} ; \phi), Q_{\Sigma}^{\mathrm{YM}}(\boldsymbol{\eta} ; \phi)\right\}=Q_{\Sigma}^{\mathrm{YM}}([\boldsymbol{\chi}, \boldsymbol{\eta}] ; \phi)$, with $\mathrm{PB}$ defined by $\boldsymbol{\Theta}_{\Sigma}^{\mathrm{YM}}$, thus representing $\operatorname{Lie} \mathcal{H}$.

Considering the question of the physical interpretation of charges (integrable or not): by the affine ansatz (3.26) $A=A_{0}+\alpha$ s.t. $D_{0} \chi \equiv 0$, i.e. $\chi$ is a Killing symmetry of the background YM field $A_{0}$, one plugs the expansion (3.27) of the field strength in the charge (3.33) which is then on-shell

$$
\begin{aligned}
Q_{\Sigma}^{\mathrm{YM}}(\chi ; \phi) & =\int_{\partial \Sigma} \theta_{\mathrm{YM}}(\chi ; \phi) \mid \mathcal{S} \\
& =\int_{\partial \Sigma} \operatorname{Tr}\left(\chi *\left\{F_{0}+f+\frac{1}{2}[\alpha, \alpha]\right\}\right), \\
& =\int_{\partial \Sigma} \operatorname{Tr}\left(\chi * F_{0}\right)+\operatorname{Tr}(\chi * f)=: Q_{\Sigma}^{\mathrm{YM}}\left(\chi ; A_{0}\right)+Q_{\Sigma}^{\mathrm{YM}}(\chi ; \alpha),
\end{aligned}
$$

where we used $\operatorname{Tr}(\chi[\alpha, \alpha])=-\operatorname{Tr}([\alpha, \chi] \alpha) \equiv 0$ by the $\mathcal{H}$-invariance of $\operatorname{Tr}(\alpha \wedge \alpha)$. The contribution $Q_{\Sigma}^{\mathrm{YM}}\left(\chi ; A_{0}\right)$ comes entirely from the background, while the second term

$$
Q_{\Sigma}^{\mathrm{YM}}(\chi ; \alpha)=\int_{\partial \Sigma} \operatorname{Tr}(\chi * f)=\int_{\partial \Sigma} * d \operatorname{Tr}(\chi \alpha)
$$

is the contribution from the perturbation $\alpha$ in the YM field, and reproduces the charge $Q_{\chi}$ of Abbott \& Deser [34] — see (B.8)-(B.9) in appendix B. All this holds still for fielddependent gauge parameters $\chi \rightarrow \chi$.

One may be interested in expressing this conserved charge in terms of the charged field $\varphi$ sourcing the YM equation. To do so, one first looks at the expansion of the field equations $D * F=J$,

$$
D_{0} * F_{0}+\left[\alpha, * F_{0}\right]+D_{0} * f+[\alpha, * f]+D^{A} * \frac{1}{2}[\alpha, \alpha]=J .
$$

Collecting on one side the terms linear in $\alpha$ and redefining a new source $j:=J-\{D * F\}^{R}$ where $\{D * F\}^{R}$ are the remaining terms, we get

$$
D_{0} * f+\left[\alpha, * F_{0}\right]=j .
$$

Together with the Killing condition $D_{0} \chi=0$, this can be used to rewrite the charge (3.39) as a bulk integral of $j$,

$$
\begin{aligned}
Q_{\Sigma}^{\mathrm{YM}}(\chi ; \alpha) & =\int_{\Sigma} d \operatorname{Tr}(\chi * f)=\int_{\Sigma} \operatorname{Tr}\left(D_{0} \chi * f+\chi D_{0} * f\right)=\int_{\Sigma} \operatorname{Tr}\left(\chi\left\{j-\left[\alpha, * F_{0}\right]\right\}\right), \\
& =\int_{\Sigma} \operatorname{Tr}(\chi j) .
\end{aligned}
$$

Where we have used $\operatorname{Tr}\left(\chi\left[\alpha, * F_{0}\right]\right)=-\operatorname{Tr}\left([\alpha, \chi] * F_{0}\right)=\operatorname{Tr}\left(\alpha\left[* F_{0}, \chi\right]\right) \equiv 0$, by the $\mathcal{H}$ invariance of $\operatorname{Tr}$ in the second equality and the Killing condition in the last.

This is the reverse of the logic of Abbott-Deser, who start with the expansion of the coupled YM equation to define the $d$-closed singlet current $\operatorname{Tr}(\chi j)$, and work so as to obtain the charge (3.39) above. As their procedure depends only on the field equations 
$\boldsymbol{E}_{\mathrm{YM}}$, it is insensitive to $L_{\mathrm{YM}}$, thus to $\boldsymbol{\theta}_{\mathrm{YM}}$. One may take advantage of this and renormalise the charge $Q_{\Sigma}^{\mathrm{YM}}(\chi ; \phi)$ by the addition of a boundary term to $L_{\mathrm{YM}}$, so as to eliminate the background contribution $Q_{\Sigma}^{\mathrm{YM}}\left(\chi ; A_{0}\right)$. The AD charge can thus be seen as a renormalisation of the Noether charge coming from the covariant phase space formalism.

For future reference, when we will consider the basic presymplectic structure of the theory, lets us finally write down the field-dependent $\mathcal{H}$-gauge transformations of the field equations and presymplectic structure. By (3.11), for $\boldsymbol{\gamma} \in \mathcal{H}=\mathcal{S U}(n)$ we get,

$$
\boldsymbol{E}_{\mathrm{YM}}^{\gamma}=\boldsymbol{E}_{\mathrm{YM}}+d E_{\mathrm{YM}}\left(\boldsymbol{d} \boldsymbol{\gamma} \boldsymbol{\gamma}^{-1} ; \phi\right)=\boldsymbol{E}+d \operatorname{Tr}\left(\boldsymbol{d} \boldsymbol{\gamma} \boldsymbol{\gamma}^{-1}\{D * F-J\}\right) .
$$

This can be verified algebraically by $\boldsymbol{E}_{\mathrm{YM}}^{\gamma}=E_{\mathrm{YM}}\left(\boldsymbol{d} \phi^{\gamma} ; \phi^{\gamma}\right)$ using (2.7) in (3.31). By (3.13)(3.17) we have immediately,

$$
\begin{aligned}
\left(\boldsymbol{\theta}_{\Sigma}^{\mathrm{YM}}\right)^{\boldsymbol{\gamma}} & =\boldsymbol{\theta}_{\Sigma}^{\mathrm{YM}}+\int_{\partial \Sigma} \theta_{\mathrm{YM}}\left(\boldsymbol{d} \boldsymbol{\gamma} \boldsymbol{\gamma}^{-1} ; \phi\right)-\int_{\Sigma} E_{\mathrm{YM}}\left(\boldsymbol{d} \boldsymbol{\gamma} \boldsymbol{\gamma}^{-1} ; \phi\right) \\
& =\boldsymbol{\theta}_{\Sigma}^{\mathrm{YM}}+\int_{\partial \Sigma} \operatorname{Tr}\left(\boldsymbol{d} \boldsymbol{\gamma} \boldsymbol{\gamma}^{-1} * F\right)-\int_{\Sigma} \operatorname{Tr}\left(\boldsymbol{d} \boldsymbol{\gamma} \boldsymbol{\gamma}^{-1}\{D * F-J\}\right) \\
\left(\boldsymbol{\Theta}_{\Sigma}^{\mathrm{YM}}\right)^{\gamma} & =\boldsymbol{\Theta}_{\Sigma}^{\mathrm{YM}}+\int_{\partial \Sigma} \boldsymbol{d} \theta_{\mathrm{YM}}\left(\boldsymbol{d} \boldsymbol{\gamma} \boldsymbol{\gamma}^{-1} ; \phi\right)-\int_{\Sigma} \boldsymbol{d} E_{\mathrm{YM}}\left(\boldsymbol{d} \boldsymbol{\gamma} \boldsymbol{\gamma}^{-1} ; \phi\right) \\
& =\boldsymbol{\Theta}_{\Sigma}^{\mathrm{YM}}+\int_{\partial \Sigma} \boldsymbol{d} \operatorname{Tr}\left(\boldsymbol{d} \boldsymbol{\gamma} \boldsymbol{\gamma}^{-1} * F\right)-\int_{\Sigma} \boldsymbol{d} \operatorname{Tr}\left(\boldsymbol{d} \boldsymbol{\gamma} \boldsymbol{\gamma}^{-1}\{D * F-J\}\right)
\end{aligned}
$$

Notice how only the Lie $\mathcal{H}$-linear pieces of $\boldsymbol{\theta}$ and $\boldsymbol{E}$ contribute to the final results. This can be verified by direct computation, using (2.7), (2.12) and (2.16) in (3.32) and (3.34). Clearly, only on-shell and under proper boundary conditions are $\boldsymbol{\theta}_{\Sigma}^{\mathrm{YM}}$ an $\boldsymbol{\Theta}_{\Sigma}^{\mathrm{YM}} \mathcal{H}$-invariant, i.e. basic forms on $\Phi$, and induce a symplectic structure on $\mathcal{M}_{\mathcal{S}}$.

Comments. Considering spinor fields $\psi$ instead of scalar fields $\varphi$ would change nothing of substance in the above results. Indeed, as we have observed several times now, only the Lie $\mathcal{H}$-linear part of $\boldsymbol{\theta}_{\mathrm{YM}}$ and $\boldsymbol{E}_{\mathrm{YM}}$ contributes to the expression of the Noether currents and charges, as well as to the $\mathcal{H}$-gauge transformations, so that the matter field contribution plays little to no role. Therefore, here the only change would be hidden in the current $J$ sourcing the gauge field, which would then be the Dirac current: $J$ can thus be treated as a black box wherein one can have any type of matter field.

For the same reason, the addition of a potential term $V(\varphi)$ for the scalar field to $L_{\mathrm{YM}}$ wouldn't affect the results presented in this section, as it just modifies the $\boldsymbol{d} \varphi$-linear part of $\boldsymbol{E}_{\mathrm{YM}}$. In particular, with the typical potential $V(\varphi)=\mu^{2}\langle\varphi, * \varphi\rangle+\lambda\langle\varphi, * \varphi\rangle^{2}$ where $\mu^{2} \in \mathbb{R}$ and $\lambda>0$, the Lagrangian becomes the prototype of a Yang-Mills-Higgs model, encompassing the electroweak model as a special case, and the above gives its charge and presymplectic structure.

\subsubsection{Coupled 4D gauge gravity}

We employ here the language of Cartan geometry [67, 68]. Meaning that we consider the underlying kinematics as given by Cartan-deSitter geometry $(P, \bar{A})$, where $P$ is a principal bundle whose structure group is $H=\mathrm{SO}(1,3)$ and whose gauge group is thus $\mathcal{H}=\mathcal{S O}(1,3)$, 
while the Cartan connection $\bar{A}$ takes value in the de Sitter/anti-de Sitter Lie algebra, $\operatorname{LieSO}(1,4)$ or $\operatorname{LieSO}(2,3)$, according to the sign of $\Lambda$.

The geometry is called reductive as we have the $H$-invariant splitting $\bar{A}=A+\frac{1}{\ell} e$, where $A=A_{b}^{a}$ is the Ehresmann Lie $H$-valued connection (the Lorentz or spin connection) and $e=e^{a}$ is the $\mathbb{R}^{4}$-valued soldering form. The Cartan curvature is thus $\bar{F}=d \bar{A}+\frac{1}{2}[\bar{A}, \bar{A}]=$ $F+\frac{1}{\ell} T=\left(R-\frac{\varepsilon}{\ell^{2}} e e^{t}\right)+\frac{1}{\ell} D^{A} e$, where $e^{t}:=e^{T} \eta=e^{a} \eta_{a b}$ and $R=d A+\frac{1}{2}[A, A]$ is the Lie $H$-valued Riemann 2-form. In matrix form,

$$
\bar{A}=\left(\begin{array}{cc}
A & \frac{1}{\ell} e \\
{[2 m m] \frac{-\varepsilon}{\ell} e^{t}} & 0
\end{array}\right), \quad \bar{F}=d \bar{A}+\bar{A}^{2}=\left(\begin{array}{cc}
F & \frac{1}{\ell} T \\
\frac{-\varepsilon}{\ell} T^{t} & 0
\end{array}\right)=\left(\begin{array}{cc}
R-\frac{\varepsilon}{\ell^{2}} e e^{t} & \frac{1}{\ell} D^{A} e \\
\frac{-\varepsilon}{\ell}\left(D^{A} e\right)^{t} & 0
\end{array}\right),
$$

with $\frac{1}{\ell^{2}}=\frac{2|\Lambda|}{(n-1)(n-2)}=\frac{|\Lambda|}{3}$ for $n=4=\operatorname{dim} \mathcal{M}$, and $\varepsilon= \pm$ is the sign of $\Lambda$. The so-called normal Cartan connection $\bar{A}_{\mid N}$ is the unique torsion-free connection, so that $A=A(e)$, meaning that the only d.o.f. in the normal connection are those of the soldering, $\bar{A}_{\mid N}=\bar{A}_{\mid N}(e) .{ }^{21}$ Cartan flatness, $\bar{F} \equiv 0$, means (in addition to vanishing torsion) $F=0 \rightarrow$ $R=\frac{\varepsilon}{\ell} e e^{t}$, that is the base manifold (spacetime) is the homogeneous de Sitter or anti-de Sitter space, $M \simeq(A) d S$.

Given the bilinear form $\eta: \mathbb{R}^{4} \times \mathbb{R}^{4} \rightarrow \mathbb{R}$, the Cartan connection induces via its soldering component a metric on $M, g:=\eta(e, e): \Gamma(T M) \times \Gamma(T M) \rightarrow \mathbb{R}$. In components $e^{a}=e^{a}{ }_{\mu} d x^{\mu}$, where $e^{a}{ }_{\mu}$ is the (co-) tetrad field, so we have the well-known relation $g_{\mu \nu}=e_{\mu}{ }^{a} \eta_{a b} e^{b}{ }_{\mu}$. To introduce notations that will be useful latter on (in section 4.3.2), let us rewrite this in the index-free fashion $\boldsymbol{e}:=e^{a}{ }_{\mu}$ and $\boldsymbol{g}=\boldsymbol{e}^{T} \eta \boldsymbol{e}$.

Dirac spinors are sections of the spin bundle $\mathbb{S}$ associated with $\mathcal{P}$ via the spin representation $\rho$ of $H=\mathrm{SO}(1,3)$ on $\mathbb{C}^{4}$. We have then $\psi \in \Gamma(\mathbb{S}) \simeq \Omega_{\mathrm{eq}}^{0}\left(\mathcal{P}, \mathbb{C}^{4}\right)$. A point of the field space $\Phi$ under consideration is $\phi=\{\bar{A}, \psi\}=\{A, e, \psi\}$ and the right action $\phi \rightarrow R_{\gamma}^{\star} \phi$ of $\mathcal{H}$ on $\Phi$ is explicitly

$$
\begin{aligned}
& R_{\gamma}^{\star} \bar{A}=\bar{A}^{\gamma}=\gamma^{-1} \bar{A} \gamma+\gamma^{-1} d \gamma \Rightarrow\left\{\begin{array}{l}
R_{\gamma}^{\star} A=A^{\gamma}=\gamma^{-1} A \gamma+\gamma^{-1} d \gamma, \\
R_{\gamma}^{\star} e=e^{\gamma}=\gamma^{-1} e,
\end{array}\right. \\
& R_{\gamma}^{\star} \psi=\psi^{\gamma}=\rho\left(\gamma^{-1}\right) \psi .
\end{aligned}
$$

It follows that, as special cases of (2.5)-(2.6), the basis $\boldsymbol{d} \phi=\{\boldsymbol{d} \bar{A}, \boldsymbol{d} \psi\}=\{\boldsymbol{d} A, \boldsymbol{d} e, \boldsymbol{d} \psi\}$ $\in \Omega_{\mathrm{eq}}^{1}(\Phi)$ is s.t.

$$
\begin{aligned}
R_{\gamma}^{\star} \boldsymbol{d} \phi & =\rho(\gamma)^{-1} \boldsymbol{d} \phi:=\left(\gamma^{-1} \boldsymbol{d} \bar{A} \gamma, \rho(\gamma)^{-1} \boldsymbol{d} \varphi\right)=\left(\gamma^{-1} \boldsymbol{d} A \gamma, \gamma^{-1} \boldsymbol{d} e, \rho(\gamma)^{-1} \boldsymbol{d} \psi\right) \\
\boldsymbol{d} \phi\left(\chi^{v}\right) & =\left(\boldsymbol{d} \bar{A}\left(\chi^{v}\right), \boldsymbol{d} \psi\left(\chi^{v}\right)\right)=\left(D^{\bar{A}} \chi,-\rho_{*}(\chi) \psi\right)=\left(D^{A} \chi,-\chi e,-\rho_{*}(\chi) \psi\right)=: \delta_{\chi} \phi
\end{aligned}
$$

\footnotetext{
${ }^{21}$ This is generically what the normality condition implies for normal Cartan connections in more elaborate situations, such as conformal Cartan geometry or more general parabolic geometries.
} 
From which follows, as a special case of (2.7), the field-dependent $\mathcal{H}$-gauge transformation on $\Phi$

$$
\boldsymbol{d} \phi^{\gamma}=\rho(\boldsymbol{\gamma})^{-1}\left(\boldsymbol{d} \phi+\delta_{\boldsymbol{d} \gamma \gamma^{-1}} \phi\right)=\left\{\begin{array}{l}
\boldsymbol{d} A^{\gamma}=\gamma^{-1}\left(\boldsymbol{d} A+D^{A}\left\{\boldsymbol{d} \boldsymbol{\gamma} \boldsymbol{\gamma}^{-1}\right\}\right) \gamma \\
\boldsymbol{d} e^{\gamma}=\gamma^{-1}\left(\boldsymbol{d} e-\boldsymbol{d} \boldsymbol{\gamma} \boldsymbol{\gamma}^{-1} e\right) \boldsymbol{\gamma} \\
\boldsymbol{d} \psi^{\gamma}=\rho(\boldsymbol{\gamma})^{-1}\left(\boldsymbol{d} \psi-\rho_{*}\left(\boldsymbol{d} \boldsymbol{\gamma} \boldsymbol{\gamma}^{-1}\right) \psi\right)
\end{array}\right.
$$

Similarly, as special instances of (2.10)-(2.12), from $R_{\gamma}^{\star} \boldsymbol{d} \bar{F}=\left(\gamma^{-1} \boldsymbol{d} F \gamma, \gamma^{-1} T\right)$ and $\boldsymbol{d} \bar{F}\left(\chi^{v}\right)=([F, \chi],-\chi T)$, we have

$$
\boldsymbol{d} \bar{F}^{\gamma}=\left\{\begin{array}{l}
\boldsymbol{d} F^{\gamma}=\gamma^{-1}\left(\boldsymbol{d} F+\left[F, \boldsymbol{d} \gamma \gamma^{-1}\right]\right) \boldsymbol{\gamma} \\
\left.\boldsymbol{d} T^{\gamma}=\gamma^{-1}\left(\boldsymbol{d} T-\boldsymbol{d} \gamma \boldsymbol{\gamma}^{-1} T\right]\right)
\end{array}\right.
$$

To write the pure gravity sector of the theory in an index-free way, we consider the multilinear polynomial $P: \otimes^{k} M(2 k, \mathbb{R}) \rightarrow \mathbb{R}$ given by

$$
P\left(A_{1}, \ldots, A_{k}\right)=A_{1} \bullet \ldots \bullet A_{k}:=A_{1}^{i_{1} i_{2}} A_{2}^{i_{3} i_{4}} \ldots A_{k}^{i_{2 k-1} i_{2 k}} \varepsilon_{i_{1} \ldots i_{2 k}},
$$

where the second equality defines the notation. Given $G \in \mathrm{GL}(2 k, \mathbb{R})$, it satisfies the identity

$$
\begin{aligned}
P\left(G A_{1}\right. & \left.G^{T}, \ldots, G A_{k} G^{T}\right) \\
& =G A_{1} G^{T} \bullet \ldots \bullet G A_{k} G^{T}, \\
& =G^{i_{1}}{ }_{j_{1}} A_{1}^{j_{1} j_{2}} G_{j_{2}}{ }^{i_{2}} G^{i_{3}}{ }_{j_{3}} A_{2}^{j_{3} j_{4}} G_{j_{4}}{ }^{i_{4}} \ldots G^{i_{2 k-1}} j_{2 k-1} A_{k}^{j_{2 k-1} j_{2 k}} G_{j_{2 k}}{ }^{i_{2 k}} \varepsilon_{i_{1} \ldots i_{2 k}}, \\
& =\operatorname{det}(G) A_{1}^{j_{1} j_{2}} A_{2}^{j_{3} j_{4}} \ldots A_{k}^{j_{2 k-1} j_{2 k}} \varepsilon_{j_{1} \ldots j_{2 k}}, \\
& =\operatorname{det}(G) A_{1} \bullet \ldots \bullet A_{k}=\operatorname{det}(G) P\left(A_{1}, \ldots, A_{k}\right) .
\end{aligned}
$$

Then, $P$ is $\mathrm{SO}(2 k)$-invariant, since for $S \in \mathrm{SO}(2 k), S^{T}=S^{-1}$, we have $P\left(S^{-1} A_{1} S, \ldots, S^{-1} A_{k} S\right)=P\left(A_{1}, \ldots, A_{k}\right)$. Also, given some matrix $M \in M(2 k, \mathbb{R})$ decomposed as the sum of its symmetric and antisymmetric parts as $M=\frac{1}{2}\left(M+M^{T}\right)+$ $\frac{1}{2}\left(M-M^{T}\right)=: S+\mathrm{A}$, we have

$$
M \bullet A_{2} \bullet \ldots \bullet A_{k}=\left(S^{i 1 i_{2}}+\mathrm{A}^{i_{1} i_{2}}\right) A_{2}^{i_{3} i_{4}} \ldots A_{k}^{i_{2 k-1} i_{2 k}} \varepsilon_{i_{1} i_{2} \ldots i_{2 k}}=\mathrm{A} \bullet A_{2} \ldots \bullet A_{k} .
$$

We have then a $\operatorname{Ad}(\mathrm{SO}(2 k))$-invariant map $P: \otimes^{k} \mathfrak{s o}(2 k) \rightarrow \mathbb{R}^{22}$ For $X \in \operatorname{LieSO}(r, s)$, $r+s=2 k, X \eta^{-1}$ is antisymmetric, and for $S \in \mathrm{SO}(r, s)$ we have $S^{-1} X S \eta^{-1}=$ $S^{-1} X \eta^{-1}\left(S^{-1}\right)^{T}$. Thus $P\left(X_{1} \eta^{-1}, \cdots, X_{k} \eta^{-1}\right)$ is a $\operatorname{Ad}(\mathrm{SO}(r, s))$-invariant polynomial $P: \otimes^{k} \mathfrak{s o}(r, s) \rightarrow \mathbb{R}$ that one can use to write the Lagrangians of even dimensional gauge gravity theories. To lighten the notation, we will omit $\eta^{-1}$ in front of $\operatorname{LieSO}(r, s)$-valued variables when writing expressions involving $P$, as it should be clear from the context that indices must be raised.

\footnotetext{
${ }^{22}$ Remark that the diagonal combination $P(A, \ldots, A)=\operatorname{Pf}(A)$ is the Pfaffian of the $2 k \times 2 k$ antisymmetric matrix $A$, which is the square root of its determinant $\operatorname{Pf}(A)^{2}=\operatorname{det}(A)$. Conversely, $P$ is the polarisation of the Pfaffian polynomial.
} 
We consider the Lagrangian of General Relativity with cosmological constant $\Lambda$, à la McDowell-Mansouri, coupled to Dirac spinors (massless to avoid unnecessary complications) to be,

$$
\begin{aligned}
L_{\mathrm{GR}}(\phi) & =L_{\mathrm{MM}}(\bar{A})+L_{\mathrm{Dirac}}(\bar{A}, \psi) \\
& =\frac{1}{2} F \bullet F+\langle\psi, \not D \psi\rangle, \\
& =\frac{1}{2} R \bullet R-\frac{\varepsilon}{\ell^{2}}\left(R \bullet e \wedge e^{T}-\frac{\varepsilon}{2 \ell^{2}} e \wedge e^{T} \bullet e \wedge e^{T}\right)+\langle\psi, \gamma \wedge * D \psi\rangle, \\
& =\frac{1}{2} R^{a b} R^{c d} \varepsilon_{a b c d}-\frac{\varepsilon}{\ell^{2}}\left(R^{a b} e^{c} e^{d}-\frac{\Lambda}{6} e^{a} e^{b} e^{c} e^{d}\right) \varepsilon_{a b c d}+\langle\psi, \gamma \wedge * D \psi\rangle .
\end{aligned}
$$

We have introduced convenient notations, and the gamma-matrices 1-form $\gamma:=\gamma_{a} e^{a}=$ $\gamma_{a} e^{a}{ }_{\mu} d x^{\mu}=: \gamma_{\mu} d x^{\mu}$ with $\left\{\gamma_{a}, \gamma_{b}\right\}=\eta_{a b} \mathbb{1}_{4}$, from which follows $\left\{\gamma_{\mu}, \gamma_{\nu}\right\}=g_{\mu \nu} \mathbb{1}_{4}$, as $g_{\mu \nu}=$ $e_{\mu}{ }^{a} \eta_{a b} e^{b}{ }_{\nu}$. It allows to define the top form $\not D \psi=\sqrt{|g|} d^{4} x \gamma_{\mu} g^{\mu \nu} D_{\nu} \psi$ on $M$. Of course, $D \psi=d \psi+\rho_{*}(A) \psi$ is the minimal coupling of spinors to gravity via the spin connection. The bilinear map $\langle\rangle:, \Gamma(\mathbb{S}) \times \Gamma(\mathbb{S}) \rightarrow \mathbb{R}$ is $\rho(H)$-invariant.

The first term in the Lagrangian is the Euler density, $L_{\text {Euler }}$, a topological invariant of $M$ : it doesn't change the field equations but contributes to the total presymplectic potential. Indeed it is easy to see that $\boldsymbol{d} L_{\text {Euler }}=\boldsymbol{E}_{\text {Euler }}+d \boldsymbol{\theta}_{\text {Euler }}=\boldsymbol{d} A \bullet D^{A} R+d(\boldsymbol{d} A \bullet R)$, and the field equations vanish identically, being just the Bianchi identity $D^{A} R \equiv 0$.

It is clear that $R_{\gamma}^{\star} L_{\mathrm{GR}}=L_{\mathrm{GR}}$ for $\gamma \in \mathcal{H}=\mathcal{S O}(1,3)$, i.e. $L_{\mathrm{GR}} \in \Omega_{\text {basic }}^{0}(\Phi)$. The only computation needed is:

$$
\begin{aligned}
\boldsymbol{d} L_{\mathrm{GR}}= & \boldsymbol{E}_{\mathrm{GR}}+d \boldsymbol{\theta}_{\mathrm{GR}} \quad \in \Omega_{\mathrm{basic}}^{1}(\Phi) \quad \text { with : } \\
\boldsymbol{E}_{\mathrm{GR}}= & -\frac{2 \varepsilon}{\ell^{2}} \boldsymbol{d} A \bullet T \wedge e^{T}+\operatorname{Tr}\left(\left|\rho_{*}(\boldsymbol{d} A) \psi\right\rangle\langle * \gamma \psi|\right) \\
& -\frac{2 \varepsilon}{\ell^{2}} \boldsymbol{d} e \wedge e^{T} \bullet\left(R-\frac{\varepsilon}{\ell^{2}} e \wedge e^{T}\right)+\boldsymbol{d} e^{a} \mathrm{~T}_{a} \\
& +\langle\boldsymbol{d} \psi, \gamma \wedge * D \psi\rangle-\langle D(* \gamma \psi), \boldsymbol{d} \psi\rangle \quad \in \Omega_{\mathrm{inv}}^{1}(\Phi) \\
\boldsymbol{\theta}_{\mathrm{GR}}= & \boldsymbol{d} A \bullet\left(R-\frac{\varepsilon}{\ell^{2}} e \wedge e^{T}\right)+\operatorname{Tr}(|\boldsymbol{d} \psi\rangle\langle * \gamma \psi|), \quad \in \Omega_{\mathrm{inv}}^{1}(\Phi) .
\end{aligned}
$$

The $\boldsymbol{d} A$-linear part of $\boldsymbol{E}_{\mathrm{GR}}$ gives the coupling of the torsion to the spin density 3-form $\mathrm{S}_{a b, c} * e^{c}=\left\langle\psi, \gamma_{c} \sigma_{a b} \psi\right\rangle * e^{c}$, where $\sigma_{a b}=\frac{1}{8}\left[\gamma_{a}, \gamma_{b}\right]$ is a representation $\left(\rho_{*}\right)$ of the basis of LieSO $(1,3)$. The $\boldsymbol{d}$-linear part of $\boldsymbol{E}_{\mathrm{GR}}$ gives of course Einstein's equations, with the stress-energy tensor 3-form $\mathrm{T}_{a}$ of the Dirac field, whose Hodge dual 1-form is $* \mathrm{~T}_{a}=\mathrm{T}_{a b} e^{b}=$ $\left(\left\langle\psi, \gamma_{a} D_{b} \psi\right\rangle-\eta_{a b} \eta^{i j}\left\langle\psi, \gamma_{i} D_{j} \psi\right\rangle\right) e^{b}$ (and coincide with the traceless canonical stress-energy tensor). The $\boldsymbol{d} \psi$-linear part of $\boldsymbol{E}_{\mathrm{GR}}$ gives Dirac's equation, $\not D \psi=0$.

By the general formula (3.6), the Noether charge associated with $\chi \in \operatorname{LieSO}(1,3)$ is,

$$
\begin{aligned}
Q_{\Sigma}^{\mathrm{GR}}(\chi ; \phi) & =\int_{\partial \Sigma} \theta_{\mathrm{GR}}(\chi ; \phi)-\int_{\Sigma} E_{\mathrm{GR}}(\chi ; \phi), \\
& =\int_{\partial \Sigma} \chi \bullet\left(R-\frac{\varepsilon}{\ell^{2}} e \wedge e^{T}\right)+\int_{\Sigma} \frac{2 \varepsilon}{\ell^{2}} \chi \bullet T \wedge e^{T}-\operatorname{Tr}\left(\left|\rho_{*}(\chi) \psi\right\rangle\langle * \gamma \psi|\right),
\end{aligned}
$$


as can be checked by direct computation. As is now usual to remark, only the Lie $H$-linear piece of $\boldsymbol{E}_{\mathrm{GR}}$ contributes, the Einstein and Dirac equations have no bearing on the result. On-shell, the charge is the same as in the pure gravity case $Q_{\Sigma}^{\mathrm{GR}}(\chi ; \phi)=Q_{\Sigma}^{\mathrm{GR}}(\chi ; \bar{A}){ }_{\mid \mathcal{S}}$ (see [33] section 5.1.4) and vanishes on the ground state of the theory, the homogeneous (anti-) de Sitter space $(A) d S$, which thus sets the zero mass-energy reference.

It would take some work to check directly what (3.7) ensures, i.e. that the presymplectic 2 -form

$$
\Theta_{\Sigma}^{\mathrm{GR}}=\int_{\Sigma} \boldsymbol{d} \boldsymbol{\theta}_{\mathrm{GR}}=-\int_{\Sigma} \boldsymbol{d} A \bullet \boldsymbol{d}\left(R-\frac{\varepsilon}{\ell^{2}} e \wedge e^{T}\right)+\operatorname{Tr}(|\boldsymbol{d} \psi\rangle\langle\boldsymbol{d}(* \gamma \psi)|), \quad \in \Omega_{\mathrm{inv}}^{1}(\Phi),
$$

relates to the charges via $\iota_{\chi^{v}} \Theta_{\Sigma}^{\mathrm{GR}}=-\boldsymbol{d} Q_{\Sigma}^{\mathrm{GR}}(\chi ; \phi)$. From which can also be verified that

$$
\left\{Q_{\Sigma}^{\mathrm{GR}}(\chi ; \phi), Q_{\Sigma}^{\mathrm{GR}}(\eta ; \phi)\right\}:=\boldsymbol{\Theta}_{\Sigma}^{\mathrm{GR}}\left(\chi^{v}, \eta^{v}\right)=Q_{\Sigma}^{\mathrm{GR}}([\chi, \eta] ; \phi),
$$

as the general formula (3.9) allows to write immediately. By (3.19), (3.23) and (3.25), the above formulae holds for field-dependent Lorentz parameters, $\chi \rightarrow \chi$, so that both Lie $\mathcal{H}$ and Lie $\mathcal{H}$ are represented faithfully by the Poisson algebra of charges, even though in the field-dependent case the Lorentz charges (3.56) are non-integrable.

Remark that, writing the charge in term of $F=R-\frac{\varepsilon}{\ell^{2}} e \wedge e^{T} \in \Omega^{2}(M, \operatorname{LieSO}(1,3))$, it is on-shell

$$
Q_{\Sigma}^{\mathrm{GR}}(\chi ; \phi)=Q_{\Sigma}^{\mathrm{GR}}(\chi ; \bar{A})=\int_{\partial \Sigma} \chi \bullet F_{\mid \mathcal{S}}=\int_{\partial \Sigma} \chi^{a b} F^{c d} \varepsilon_{a b c d} .
$$

The striking similarity with the YM case is no surprise as we wrote the pure gravity sector à la McDowell-Mansouri, $L_{\mathrm{MM}}(\bar{A})=\frac{1}{2} F \bullet F=\frac{1}{2} F^{a b} F^{c d} \varepsilon_{a b c d}$. Thus, the question of the physical interpretation of charges can be answered in essentially the same terms as in YM theory. One first make the affine ansatz (3.26) $\bar{A}=\bar{A}_{0}+\bar{\alpha}$ s.t. $D_{0} \chi:=D^{\bar{A}_{0}} \chi \equiv 0$, i.e. $\chi$ is a Killing symmetry of the background gravitational field (Cartan connection) $\bar{A}_{0}=A_{0}+e_{0}$, while $\bar{\alpha}=\alpha+\epsilon$ is a perturbation. From it follows the analogue of expansion (3.27) for the Cartan curvature, $\bar{F}=\bar{F}_{0}+\bar{f}+\frac{1}{2}[\bar{\alpha}, \bar{\alpha}]$, whose Lorentz component gives

$$
F=F_{0}+\left(D^{A_{0}} \alpha-\frac{\varepsilon}{\ell^{2}}\left(e_{0} \wedge \epsilon^{t}+\epsilon \wedge e_{0}^{t}\right)\right)+\left(\frac{1}{2}[\alpha, \alpha]-\frac{\varepsilon}{\ell^{2}} \epsilon \wedge \epsilon^{t}\right)=: F_{0}+f+\frac{1}{2}[\alpha, \alpha] .
$$

Plugging this into the charge (3.56), it becomes on-shell

$$
\begin{aligned}
Q_{\Sigma}^{\mathrm{GR}}(\chi ; \phi) & =\int_{\partial \Sigma} \chi \bullet\left(F_{0}+f+\frac{1}{2}[\alpha, \alpha]\right), \\
& =\int_{\partial \Sigma} \chi \bullet F_{0}+\chi \bullet f=: Q_{\Sigma}^{\mathrm{GR}}\left(\chi ; \bar{A}_{0}\right)+Q_{\Sigma}^{\mathrm{GR}}(\chi ; \bar{\alpha}),
\end{aligned}
$$

where we used $\chi \bullet[\alpha, \alpha]=[\alpha, \chi] \bullet \alpha \equiv 0$ by the $\mathcal{H}$-invariance of $\alpha \bullet \alpha$. Here again, $Q_{\Sigma}^{\mathrm{GR}}(\chi ; \bar{\alpha})$ is the charge measured against the background $\bar{A}_{0}$, which in particular can chosen to be the (A)dS groundstate so that $Q_{\Sigma}^{\mathrm{GR}}\left(\chi ; \bar{A}_{0}\right) \equiv 0$. 
Finally, for future expression of the basic presympletic structure, let us write the field-dependent transformations of the field equations and presymplecture structure of the theory. By (3.11), (3.13) and (3.17), for $\boldsymbol{\gamma} \in \mathcal{H}=\mathcal{S O}(1,3)$ :

$$
\begin{aligned}
\boldsymbol{E}_{\mathrm{GR}}^{\gamma}= & \boldsymbol{E}_{\mathrm{GR}}+d E_{\mathrm{GR}}\left(\boldsymbol{d} \boldsymbol{\gamma} \boldsymbol{\gamma}^{-1} ; \phi\right) \\
= & \boldsymbol{E}_{\mathrm{GR}}-d\left\{\frac{2 \varepsilon}{\ell^{2}}\left(\boldsymbol{d} \boldsymbol{\gamma} \boldsymbol{\gamma}^{-1} \bullet T \wedge e^{T}\right)-\operatorname{Tr}\left(\left|\rho_{*}\left(\boldsymbol{d} \boldsymbol{\gamma} \boldsymbol{\gamma}^{-1}\right) \psi\right\rangle\langle * \gamma \psi|\right)\right\} \\
\left(\boldsymbol{\theta}_{\Sigma}^{\mathrm{GR}}\right)^{\gamma}= & \boldsymbol{\theta}_{\Sigma}^{\mathrm{GR}}+\int_{\partial \Sigma} \theta_{\mathrm{GR}}\left(\boldsymbol{d} \boldsymbol{\gamma} \boldsymbol{\gamma}^{-1} ; \phi\right)-\int_{\Sigma} E_{\mathrm{GR}}\left(\boldsymbol{d} \boldsymbol{\gamma} \boldsymbol{\gamma}^{-1} ; \phi\right) \\
= & \boldsymbol{\theta}_{\Sigma}^{\mathrm{GR}}+\int_{\partial \Sigma} \boldsymbol{d} \boldsymbol{\gamma} \boldsymbol{\gamma}^{-1} \bullet F+\int_{\Sigma} \frac{2 \varepsilon}{\ell^{2}} \boldsymbol{d} \boldsymbol{\gamma} \boldsymbol{\gamma}^{-1} \bullet T \wedge e^{T}-\operatorname{Tr}\left(\left|\rho_{*}\left(\boldsymbol{d} \boldsymbol{\gamma} \boldsymbol{\gamma}^{-1}\right) \psi\right\rangle\langle * \gamma \psi|\right) \\
\left(\boldsymbol{\Theta}_{\Sigma}^{\mathrm{GR}}\right)^{\boldsymbol{\gamma}}= & \boldsymbol{\Theta}_{\Sigma}^{\mathrm{GR}}+\int_{\partial \Sigma} \boldsymbol{d} \theta_{\mathrm{GR}}\left(\boldsymbol{d} \boldsymbol{\gamma} \boldsymbol{\gamma}^{-1} ; \phi\right)-\int_{\partial \Sigma} \boldsymbol{d} E_{\mathrm{GR}}\left(\boldsymbol{d} \boldsymbol{\gamma} \boldsymbol{\gamma}^{-1} ; \phi\right) \\
= & \boldsymbol{\Theta}_{\Sigma}^{\mathrm{GR}}+\int_{\partial \Sigma} \boldsymbol{d}\left(\boldsymbol{d} \boldsymbol{\gamma} \boldsymbol{\gamma}^{-1} \bullet F\right)+\int_{\Sigma} \frac{2 \varepsilon}{\ell^{2}} \boldsymbol{d}\left(\boldsymbol{d} \boldsymbol{\gamma} \boldsymbol{\gamma}^{-1} \bullet T \wedge e^{T}\right) \\
& -\boldsymbol{d} \operatorname{Tr}\left(\left|\rho_{*}\left(\boldsymbol{d} \boldsymbol{\gamma} \boldsymbol{\gamma}^{-1}\right) \psi\right\rangle\langle * \gamma \psi|\right)
\end{aligned}
$$

which can be checked by explicit computation, with some work, via (3.48)-(3.49) and (2.16) (which holds for $\varphi \rightarrow \psi$ ). Remark that for solutions of the theory that asymptotically decay to the $(A) d S$ ground state, $\bar{F}=0$, both $\boldsymbol{\theta}_{\Sigma}^{\mathrm{GR}}$ and $\boldsymbol{\Theta}_{\Sigma}^{\mathrm{GR}}$ are $\mathcal{S} \mathcal{O}(1,3)$-invariant, and thus induce respectively a symplectic potential and 2-form on the physical phase space $\mathcal{M}_{\mathcal{S}}$.

\section{Basic presymplectic structures}

As we have seen, the non-horizontality of $\boldsymbol{\theta}$ and $\boldsymbol{\Theta}$ is crucial to the very definition of Noether currents and charges (3.5)-(3.6), and to the construction of the associated Poisson bracket (3.7)-(3.9). But is also results in their non-trivial $\mathcal{H}$-gauge transformations (3.13)(3.17), which is a problem regarding the goal of associating a symplectic structure to a gauge theory over a bounded region. This lack of horizontality, thus of basicity, of $\boldsymbol{\theta}$ and $\boldsymbol{\Theta}$ is what we have called the boundary problem.

Yet, in the case at hand $L \in \Omega_{\text {basic }}^{0}(\Phi)$, thus $\boldsymbol{d} L \in \Omega_{\text {basic }}^{1}(\Phi)$. This means first that $\exists \bar{L} \in \Omega^{0}(\mathcal{M})$ s.t. $L=\pi^{\star} \bar{L}$, by definition of a basic form. Then, by the same argument leading to (3.3), on $\mathcal{M}$ we have $\boldsymbol{d}_{\mathcal{M}} \bar{L}=\overline{\boldsymbol{E}}+d \overline{\boldsymbol{\theta}}$, with $\overline{\boldsymbol{E}}, \overline{\boldsymbol{\theta}} \in \Omega^{1}(\mathcal{M})$. Therefore, by naturality and linearity of the pullback we must have

$$
\begin{aligned}
& \boldsymbol{d} L=\boldsymbol{d} \pi^{\star} \bar{L}=\pi^{\star} \boldsymbol{d}_{\mathcal{M}} \bar{L}=\pi^{\star}(\overline{\boldsymbol{E}}+d \overline{\boldsymbol{\theta}})=\pi^{\star} \overline{\boldsymbol{E}}+d\left(\pi^{\star} \overline{\boldsymbol{\theta}}\right)=: \boldsymbol{E}^{b}+d \boldsymbol{\theta}^{b}, \\
& \text { with } \boldsymbol{E}^{b}, \boldsymbol{\theta}^{b} \in \Omega_{\mathrm{basic}}^{1}(\Phi) .
\end{aligned}
$$

It would thus seem that we should be able to define a basic presymplectic potential $\boldsymbol{\theta}^{b}$, from which to naturally derive a basic presymplectic 2-form $\boldsymbol{\Theta}^{b}:=\boldsymbol{d} \boldsymbol{\theta}^{b} \in \Omega_{\text {basic }}^{2}(\Phi)$ - since $\boldsymbol{d}$ is the covariant derivative on basic forms, i.e. preserves the space. But how are we to find such a basic presymplectic structure starting from the known $\boldsymbol{\theta}$ and $\boldsymbol{\Theta}$ ? In sections 2.2 and 2.3 we have already seen two methods to do so, using respectively variational connections or the DFM. In the following, we consider the results of each approach in turn. 


\subsection{Via variational connections}

We use the general results of section 2.2.1 about the dual horizontalisation of forms relying on a variational Ehresmann connection $\boldsymbol{\omega}$. When a Lagrangian $L$ is invariant so are its associated field equations and presymplectic potential $\boldsymbol{E}, \boldsymbol{\theta} \in \Omega_{\mathrm{inv}}^{1}(\Phi)$, eq. (3.4). So, applying the formula (2.23), and using first $\iota_{\chi^{v}} \boldsymbol{E}=d E(\chi ; \phi)-$ stemming from $\boldsymbol{L}_{\chi^{v}} L=0$ and (3.5) — we get the basic field equations:

$$
\boldsymbol{E}_{\boldsymbol{\omega}}^{b}=\boldsymbol{E}-d E(\boldsymbol{\omega} ; \phi) \quad \in \Omega_{\text {basic }}^{1}(\Phi),
$$

By (2.23) still, and given (3.5), we get the $\boldsymbol{\omega}$-dependent basic presymplectic potential,

$$
\begin{aligned}
\boldsymbol{\theta}_{\boldsymbol{\omega}}^{b} & =\boldsymbol{\theta}-J(\boldsymbol{\omega} ; \phi), \quad \in \Omega_{\text {basic }}^{1}(\Phi), \\
& =\boldsymbol{\theta}-d \theta(\boldsymbol{\omega} ; \phi)+E(\boldsymbol{\omega} ; \phi) .
\end{aligned}
$$

The $\mathcal{H}$-invariance of both $\boldsymbol{E}_{\omega}^{b}$ and $\boldsymbol{\theta}_{\boldsymbol{\omega}}^{b}$, although structurally garanteed, is easily checked explicitly knowing (3.11)-(3.12) and $\boldsymbol{\omega}^{\boldsymbol{\gamma}}=\boldsymbol{\gamma}^{-1} \boldsymbol{\omega} \boldsymbol{\gamma}+\boldsymbol{\gamma}^{-1} \boldsymbol{d} \boldsymbol{\gamma}$. The basic presymplectic 2-form naturally associated with $\boldsymbol{\theta}_{\boldsymbol{\omega}}^{b}$ is then,

$$
\begin{aligned}
\boldsymbol{\Theta}_{\boldsymbol{\omega}}^{b} & :=\boldsymbol{d} \boldsymbol{\theta}_{\boldsymbol{\omega}}^{b} \quad \in \Omega_{\text {basic }}^{2}(\Phi), \\
& =\boldsymbol{\Theta}-\boldsymbol{d} J(\boldsymbol{\omega} ; \phi), \\
& =\boldsymbol{\Theta}-\boldsymbol{d}(d \theta(\boldsymbol{\omega} ; \phi)-E(\boldsymbol{\omega} ; \phi)),
\end{aligned}
$$

whose $\mathcal{H}$-invariance is again easily checked via (3.16). We may remark that contrary to what one could be tempted to do, the correct approach is not to build the horizontal version of $\boldsymbol{\Theta} \in \Omega_{\text {inv }}^{2}(\Phi)$, as it is actually by definition the covariant derivative - w.r.t. $\boldsymbol{\omega}$ of $\boldsymbol{\theta} \in \Omega_{\text {inv }}^{1}(\Phi)$ :

$$
\boldsymbol{\Theta}^{h}:=\left.\boldsymbol{\Theta} \circ\right|^{h}:=\left.\boldsymbol{d} \boldsymbol{\theta} \circ\right|^{h}=: \boldsymbol{D}^{\boldsymbol{\omega}} \boldsymbol{\theta}, \quad \in \Omega_{\text {basic }}^{2}(\Phi) .
$$

If it is basic indeed, it is not $\boldsymbol{d}$-closed, so cannot play the role of a presymplectic form. Actually, specialising eq. (2.27) to this case and using again (3.5), we get

$$
\begin{aligned}
\boldsymbol{D}^{\omega} \boldsymbol{\theta} & =\boldsymbol{d} \boldsymbol{\theta}_{\boldsymbol{\omega}}^{b}+\iota_{[\boldsymbol{\Omega}]^{v}} \boldsymbol{\theta}, \\
\boldsymbol{\Theta}^{h} & =\boldsymbol{\Theta}_{\boldsymbol{\omega}}^{b}+J(\boldsymbol{\Omega} ; \phi), \\
& =\boldsymbol{\Theta}_{\boldsymbol{\omega}}^{b}+d \theta(\boldsymbol{\Omega} ; \phi)-E(\boldsymbol{\Omega} ; \phi),
\end{aligned}
$$

where of course $\boldsymbol{\Omega} \in \Omega_{\text {tens }}^{2}(\Phi)$ is the curvature of $\boldsymbol{\omega}$. Thus, the actual basic presymplectic 2 -form is the $\boldsymbol{d}$-exact part of the covariant derivative of the presymplectic potential $\boldsymbol{\theta}$. The above formula generalises the remark already made by Gomes \& Riello in the YM case - [9], corollary 3.2 and section 3.4, see also [13] end of section 3.1. Manifestly, for a flat connections $\stackrel{\circ}{\omega}$ the situation is degenerate, $\stackrel{\circ}{\Theta}^{h}:=\boldsymbol{D}^{\stackrel{\dot{\omega}}{\theta}}=\boldsymbol{\Theta}_{\stackrel{\omega}{\omega}}^{b}$ (this is relevant to our discussion of the approach via the DFM).

The basic presymplectic structure is then given by

$$
\begin{aligned}
& \boldsymbol{\theta}_{\boldsymbol{\omega}, \Sigma}^{b}=\boldsymbol{\theta}_{\Sigma}-\int_{\partial \Sigma} \theta(\boldsymbol{\omega} ; \phi)+\int_{\Sigma} E(\boldsymbol{\omega} ; \phi), \quad \in \Omega_{\text {basic }}^{1}(\Phi), \\
& \boldsymbol{\Theta}_{\boldsymbol{\omega}, \Sigma}^{b}=\boldsymbol{\Theta}_{\Sigma}-\int_{\partial \Sigma} \boldsymbol{d} \theta(\boldsymbol{\omega} ; \phi)+\int_{\Sigma} \boldsymbol{d} E(\boldsymbol{\omega} ; \phi), \quad \in \Omega_{\text {basic }}^{2}(\Phi) .
\end{aligned}
$$


which descend to $\mathcal{M}$, and on-shell turn $\mathcal{M}_{\mathcal{S}}$ into the desired reduced phase space associated with the gauge theory $L$ over $\Sigma$. The presence of a boundary is no longer a problem.

Notice how in the above construction of basic forms, only the Lie $\mathcal{H}$-linear part of $\boldsymbol{E}$ (and $\boldsymbol{\theta}$ ) contributes, so that the field equations for the matter fields are irrelevant to the scheme. ${ }^{23}$

Ambiguity in the choice of connection. As the notation suggests though, the use of a connection $\boldsymbol{\omega}$ makes (4.2), (4.3) and (4.4) "coordinatisations" of the abstract basic objects $\boldsymbol{E}^{b}, \boldsymbol{\theta}^{b}$ and $\boldsymbol{\Theta}^{b}$. By the work done at the end of section 2.2.1, we easily find what happens under change of coordinatisation, i.e. under change of variational connection. As a special case of equation (2.30), and using again (3.5), we have that basic presymplectic potentials built from connections $\boldsymbol{\omega}$ and $\boldsymbol{\omega}^{\prime}$ s.t. $\boldsymbol{\omega}^{\prime}=\boldsymbol{\omega}+\boldsymbol{\beta}$ with $\boldsymbol{\beta} \in \Omega_{\text {tens }}^{1}(\Phi$, Lie $\mathcal{H})$, are related as

$$
\begin{aligned}
\boldsymbol{\theta}_{\boldsymbol{\omega}^{\prime}}^{b} & =\boldsymbol{\theta}_{\boldsymbol{\omega}}^{b}-J(\boldsymbol{\beta} ; \phi), \\
& =\boldsymbol{\theta}_{\boldsymbol{\omega}}^{b}-d \theta(\boldsymbol{\beta} ; \phi)+E(\boldsymbol{\beta} ; \phi) . \\
\text { so that } \quad \boldsymbol{\theta}_{\omega^{\prime}, \Sigma}^{b} & =\boldsymbol{\theta}_{\boldsymbol{\omega}, \Sigma}^{b}-\int_{\partial \Sigma} \theta(\boldsymbol{\beta} ; \phi)+\int_{\Sigma} E(\boldsymbol{\beta} ; \phi) .
\end{aligned}
$$

Similarly for basic field equations 1-forms,

$$
\boldsymbol{E}_{\omega^{\prime}}^{b}=\boldsymbol{E}_{\omega}^{b}-d E(\boldsymbol{\beta} ; \phi)
$$

As a special case of (2.31), and following directly from (4.8), basic presymplectic 2-forms are related as,

$$
\begin{aligned}
\boldsymbol{\Theta}_{\omega^{\prime}}^{b} & =\boldsymbol{\Theta}_{\omega}^{b}-\boldsymbol{d} J(\boldsymbol{\beta} ; \phi), \\
& =\boldsymbol{\Theta}_{\omega}^{b}-\boldsymbol{d}(d \theta(\boldsymbol{\beta} ; \phi)-E(\boldsymbol{\beta} ; \phi)) . \\
\text { so that } \quad \boldsymbol{\Theta}_{\boldsymbol{\omega}^{\prime}, \Sigma}^{b} & =\boldsymbol{\Theta}_{\boldsymbol{\omega}, \Sigma}^{b}-\int_{\partial \Sigma} \boldsymbol{d} \theta(\boldsymbol{\beta} ; \phi)+\int_{\Sigma} \boldsymbol{d} E(\boldsymbol{\beta} ; \phi) .
\end{aligned}
$$

The ambiguity relations (4.8)-(4.10) stems from the affine structure of the space of variational connections. It can be reasonably neglected if a preferred choice is available. Such would be the case in pure gauge theories since, as discussed in section 2.2.1, $\mathcal{A}$ has a distinguished connection $\boldsymbol{\omega}^{\stackrel{̊}{g}}$ associated with a natural bundle metric $\stackrel{\circ}{g}$ (called the Singer-deWitt connection by Gomes-Riello).

These ambiguity relations could also be interpreted as reflecting gluing properties: if one imagines that observers on regions $\Sigma^{\prime}$ and $\Sigma$ separated by a boundary $\partial \Sigma$ use different variational connections to build their respective basic presymplectic structures, then (4.8)(4.10) - with $\Sigma$ on the left-hand side replaced by $\Sigma^{\prime}$ - are gluing relations between these structures. Thus understood, the above results generalise the discussion of section 6.7 in [9] on gluings of basic Yang-Mills presymplectic potentials built via Singer-deWitt connections.

\footnotetext{
${ }^{23}$ We could see this as another hint supporting the conceptual primacy/priority of the principal bundle $\mathcal{P}$ - hence of gauge interactions — over all associated bundles - i.e. the matter fields.
} 


\subsection{Via dressing fields}

We use the general results of section 2.3 on the construction of basic forms relying on a field-dependent dressing fields $\boldsymbol{u}$. Applying the general formula (2.48), and using our rule of thumb explained around (2.50) together with the results we obtained in section 3.2 for the $\mathcal{H}$-gauge transformations of the field equations (3.11), the presymplectic potential (3.12)(3.13) and the presymplectic 2-form (3.16)-(3.17), we immediately get their associated dressed basic forms. First the dressed field equations,

$$
\boldsymbol{E}^{\boldsymbol{u}}=\boldsymbol{E}+d E\left(\boldsymbol{d u u ^ { - 1 }} ; \phi\right) \quad \in \Omega_{\text {basic }}^{1}(\Phi) .
$$

Then the dressed presymplectic structure,

$$
\begin{aligned}
& \boldsymbol{\theta}_{\Sigma}^{\boldsymbol{u}}=\boldsymbol{\theta}_{\Sigma}+\int_{\partial \Sigma} \theta\left(\boldsymbol{d u u ^ { - 1 }} ; \phi\right)-\int_{\Sigma} E\left(\boldsymbol{d u u ^ { - 1 }} ; \phi\right), \quad \in \Omega_{\mathrm{basic}}^{1}(\Phi), \\
& \boldsymbol{\Theta}_{\Sigma}^{\boldsymbol{u}}=\boldsymbol{\Theta}_{\Sigma}+\int_{\partial \Sigma} \boldsymbol{d} \theta\left(\boldsymbol{d u u ^ { - 1 }} ; \phi\right)-\int_{\Sigma} \boldsymbol{d} E\left(\boldsymbol{d u u ^ { - 1 }} ; \phi\right), \quad \in \Omega_{\mathrm{basic}}^{2}(\Phi) .
\end{aligned}
$$

As with (4.2)-(4.7), these can be seen as realisations of the basic $\boldsymbol{E}^{b}, \boldsymbol{\theta}_{\Sigma}^{b}$ and $\boldsymbol{\Theta}_{\Sigma}^{b}$ associated with the invariant Lagrangian $L$ - as suggested in the introduction to section 4 . But a complementary viewpoint, central to the DFM philosophy, is available and worth stressing: (4.11)-(4.12) are the field equations and presymplectic structure associated with the dressed Lagrangian

$$
L^{u}:=\mathrm{F}_{\boldsymbol{u}}^{\star} L=L \circ \mathrm{F}_{\boldsymbol{u}} \quad \in \Omega_{\text {basic }}^{0}(\Phi), \quad \text { i.e. } \quad L^{\boldsymbol{u}}(\phi)=L\left(\phi^{\boldsymbol{u}}\right),
$$

and obtained in the standard way from $\boldsymbol{d} L^{\boldsymbol{u}}=\boldsymbol{E}^{\boldsymbol{u}}+d \boldsymbol{\theta}^{u}$, and $\boldsymbol{\Theta}^{\boldsymbol{u}}=\boldsymbol{d} \boldsymbol{\theta}^{\boldsymbol{u}}$.

$$
\boldsymbol{d} L^{\boldsymbol{u}}=\boldsymbol{E}^{\boldsymbol{u}}+d \boldsymbol{\theta}^{\boldsymbol{u}}=E\left(\boldsymbol{d} \phi^{\boldsymbol{u}} ; \phi^{\boldsymbol{u}}\right)+d \theta\left(\boldsymbol{d} \phi^{\boldsymbol{u}} ; \phi^{\boldsymbol{u}}\right) \quad \in \Omega_{\mathrm{basic}}^{1}(\Phi) .
$$

The latter expression would allow to cross-check algebraically (4.11)-(4.12) by inserting (2.49) and (2.45)-(2.40) in $E$ and $\theta$.

This viewpoint is relevant to the question of ambiguity in the choice of dressing, as we are about to discuss. But let us also remark that it clarifies the meaning of the edge mode strategy as introduced by Donnelly \& Freidel [5], and applied in various contexts since [1417, 23-25, 32], where dressing fields are known as 'edge modes'. As argued in [33], the DFM is the geometric foundation of this strategy. Taking indeed (4.12) on-shell, it may seem that $\boldsymbol{u}$ needs only to live on $\partial \Sigma$, hence the name 'edge mode' it received elsewhere. But considering the boundary as a fictitious one, being arbitrarily moved around, we see that actually $\boldsymbol{u}$ must in general be defined across $\Sigma$. This indeed makes all the more sense considering that $\boldsymbol{u}$ is built from $\phi$ which is defined across $\Sigma$.

The striking similarity between (4.11)-(4.12) and (4.2)-(4.7) is of course no accident. As we observed in section 2.3.1, the 1 -form $\stackrel{\circ}{\boldsymbol{\omega}}:=-\boldsymbol{d u} \boldsymbol{u}^{-1}$ is a flat variational Ehresmann connection. So, regarding the question of realising the basic presymplectic structure of invariant gauge theories, the dressed structure (4.11)-(4.12) can be seen as a special case of (4.2)-(4.7) involving a flat connection. This generalises the observation of GomesRiello [7] according to which the edge mode strategy of Donnelly-Freidel as applied to YM theory could be seen as a special case of their use of a connection. 
Yet, there is a noticeable difference in what can be done via the DFM that wouldn't be accessible through the use of a variational connection, and this relates to how ambiguities in the respective schemes arise.

Ambiguity in the choice of dressing field and residual $\mathcal{G}$-transformations. Again, as the notation suggests, (4.11)-(4.12) are "coordinatisations" of the abstract basic objects $\boldsymbol{E}^{b}, \boldsymbol{\theta}^{b}$ and $\boldsymbol{\Theta}^{b}$. From sections 2.3 and 2.3.1, we know what happens under change of coordinatisation, i.e. under change of dressing field $\boldsymbol{u}^{\prime}, \boldsymbol{u}: \Phi \rightarrow \mathcal{D} r[H, G]$.

We have already seen that such a change is, in the most general case, of the form $\boldsymbol{u}^{\prime}=\boldsymbol{u} \boldsymbol{\xi}$ for some $\phi$-dependent $\mathcal{G}$-valued map $\boldsymbol{\xi}$ s.t. $R_{\gamma}^{\star} \boldsymbol{\xi}=\boldsymbol{\xi}$. By application of $(2.59)$ (or $(2.55)$ ), we immediately get the relations

$$
\begin{aligned}
\left(\boldsymbol{E}^{\boldsymbol{u}}\right)^{\boldsymbol{\xi}} & =\boldsymbol{E}^{u}-d E(\stackrel{\circ}{\boldsymbol{\beta}} ; \phi), \\
\left(\boldsymbol{\theta}_{\Sigma}^{u}\right)^{\xi} & =\boldsymbol{\theta}_{\Sigma}^{u}-\int_{\partial \Sigma} \theta(\stackrel{\circ}{\boldsymbol{\beta}} ; \phi)+\int_{\Sigma} E(\stackrel{\circ}{\boldsymbol{\beta}} ; \phi),
\end{aligned}
$$

with $\stackrel{\circ}{\boldsymbol{\beta}}=-\boldsymbol{u} \boldsymbol{d} \boldsymbol{\xi} \boldsymbol{\xi}^{-\mathbf{1}} \boldsymbol{u}^{-1} \in \Omega_{\text {tens }}^{1}(\Phi)$. From which follows,

$$
\left(\boldsymbol{\Theta}_{\Sigma}^{u}\right)^{\xi}=\Theta_{\Sigma}^{u}-\int_{\partial \Sigma} \boldsymbol{d} \theta(\stackrel{\circ}{\boldsymbol{\beta}} ; \phi)+\int_{\Sigma} \boldsymbol{d} E(\stackrel{\circ}{\boldsymbol{\beta}} ; \phi) .
$$

These can of course be interpreted as special cases of (4.8)-(4.10) since change of dressing fields reflects a case of the affine character of connection space, $\stackrel{\circ}{\omega}^{\prime}=\stackrel{\circ}{\boldsymbol{\omega}}+\stackrel{\circ}{\boldsymbol{\beta}}$. But there is more to it.

In section 2.3, we made the case that dressed variational forms $\boldsymbol{\alpha}^{u}$, basic on $\Phi$, can be seen as forms on the $\mathcal{G}$-bundle of dressed fields $\Phi^{u}$, arising from the ambiguity in the choice of dressing. From that point of view, the above relations are transformations under the gauge group $\mathcal{G}$ of $\Phi^{u}$ - remember the SES (2.43). Recalling indeed that the first version of eq. (2.55)-(2.59) is the general equation (2.53), we apply the latter to rewrite (4.15)$(4.16)$ as

$$
\begin{aligned}
\left(\boldsymbol{E}^{\boldsymbol{u}}\right)^{\xi} & =\boldsymbol{E}^{\boldsymbol{u}}+d E\left(\boldsymbol{d} \boldsymbol{\xi} \boldsymbol{\xi}^{-\mathbf{1}} ; \phi^{\boldsymbol{u}}\right), \\
\left(\boldsymbol{\theta}_{\Sigma}^{\boldsymbol{u}}\right)^{\boldsymbol{\xi}} & =\boldsymbol{\theta}_{\Sigma}^{\boldsymbol{u}}+\int_{\partial \Sigma} \theta\left(\boldsymbol{d} \boldsymbol{\xi} \boldsymbol{\xi}^{-\mathbf{1}} ; \phi^{\boldsymbol{u}}\right)-\int_{\Sigma} E\left(\boldsymbol{d} \boldsymbol{\xi} \boldsymbol{\xi}^{-\mathbf{1}} ; \phi^{\boldsymbol{u}}\right), \\
\left(\boldsymbol{\Theta}_{\Sigma}^{\boldsymbol{u}}\right)^{\boldsymbol{\xi}} & =\boldsymbol{\Theta}_{\Sigma}^{\boldsymbol{u}}+\int_{\partial \Sigma} \boldsymbol{d} \theta\left(\boldsymbol{d} \boldsymbol{\xi} \boldsymbol{\xi}^{-\mathbf{1}} ; \phi^{\boldsymbol{u}}\right)-\int_{\Sigma} \boldsymbol{d} E\left(\boldsymbol{d} \boldsymbol{\xi} \boldsymbol{\xi}^{-\mathbf{1}} ; \phi^{\boldsymbol{u}}\right) .
\end{aligned}
$$

which, of course, look exactly like the $\mathcal{H}$-transformations of $\boldsymbol{E}$ (3.11), $\boldsymbol{\theta}_{\Sigma}$ (3.13) and $\Theta_{\Sigma}$ (3.17). What is especially interesting though is that since the dressing ambiguity is encoded by a transformation group $\mathcal{G}$, which is the structure group of the bundle $\Phi^{u}$, associated Noether charges and their Poisson bracket can be defined.

Indeed, as by (2.42) we have that $R_{\xi} \phi^{u}, \xi \in \mathcal{G}$ is formally identical to $R_{\gamma} \phi, \gamma \in \mathcal{H}$, and since obviously $L$ and $L^{\boldsymbol{u}}$ have the same functional properties, among which invariance, it follows that $R_{\xi}^{\star} L^{u}=L^{u}$, i.e. $L^{u} \in \Omega_{\text {basic }}^{0}\left(\Phi^{u}\right)$. Therefore, $d L^{u} \in \Omega_{\text {basic }}^{1}\left(\Phi^{u}\right)$ and on $\Phi^{u}$ we get,

$$
\boldsymbol{d} L^{\boldsymbol{u}}=\boldsymbol{E}^{u}+d \boldsymbol{\theta}^{\boldsymbol{u}}=E\left(\boldsymbol{d} \phi^{\boldsymbol{u}} ; \phi^{\boldsymbol{u}}\right)+d \theta\left(\boldsymbol{d} \phi^{\boldsymbol{u}} ; \phi^{\boldsymbol{u}}\right) .
$$


From which is defined $\Theta^{u}:=\boldsymbol{d} \boldsymbol{\theta}^{u}$. From there, the whole covariant phase space approach on $\Phi^{u}$ can be run through as in section 3.2. We immediately get to write down the dressed Noether charges associate to $\varkappa \in$ Lie $\mathcal{G}$, generating the vertical vector fields $\varkappa^{v} \in \Gamma\left(V \Phi^{u}\right)$ :

$$
Q_{\Sigma}\left(\varkappa ; \phi^{u}\right)=\int_{\partial \Sigma} \theta\left(\varkappa ; \phi^{u}\right)-\int_{\Sigma} E\left(\varkappa ; \phi^{u}\right) .
$$

These are related to the dressed presymplectic 2-form as $\iota_{\varkappa v} \boldsymbol{\Theta}_{\Sigma}^{\boldsymbol{u}}=-\boldsymbol{d} Q_{\Sigma}\left(\varkappa ; \phi^{\boldsymbol{u}}\right)-\varkappa$ is field-independent so the charges are integrable - so that a Poisson bracket is defined the usual way:

$$
\left\{Q_{\Sigma}\left(\varkappa ; \phi^{u}\right), Q_{\Sigma}\left(\varkappa^{\prime} ; \phi^{u}\right)\right\}:=\Theta_{\Sigma}^{u}\left(\varkappa^{v}, \varkappa^{\prime v}\right)=Q_{\Sigma}\left(\left[\varkappa, \varkappa^{\prime}\right] ; \phi^{u}\right) .
$$

The Poisson algebra of dressed Noether charges is then isomorphic to Lie $\mathcal{G}$, and infinitesimal $\mathcal{G}$-transformations (of objects on $\Phi^{\boldsymbol{u}}$ ) can be generated via $\left\{Q_{\Sigma}\left(\alpha ; A^{\boldsymbol{u}}\right), \quad\right\}$.

Equations (4.17) are, as we said, $\phi^{u}$-dependent $\mathcal{G}$-gauge transformations. They are used to extend the above to dressed charges associated with $\varkappa \in$ Lie $\mathcal{G}$, for which we get

$$
\begin{aligned}
& Q_{\Sigma}\left(\varkappa ; \phi^{\boldsymbol{u}}\right)=\int_{\partial \Sigma} \theta\left(\varkappa ; \phi^{\boldsymbol{u}}\right)-\int_{\Sigma} E\left(\varkappa ; \phi^{\boldsymbol{u}}\right) \\
& \text { which are s.t. } \iota_{\varkappa^{v}} \boldsymbol{\Theta}_{\Sigma}^{\boldsymbol{u}}=-\boldsymbol{d} Q_{\Sigma}\left(\varkappa ; \phi^{\boldsymbol{u}}\right)+\boldsymbol{d} Q_{\Sigma}\left(\boldsymbol{d} \varkappa ; \phi^{\boldsymbol{u}}\right) \text {, } \\
& =-\boldsymbol{d} Q_{\Sigma}\left(\underline{\varkappa} ; \phi^{\boldsymbol{u}}\right) \text {. }
\end{aligned}
$$

These non-integrable dressed charges still satisfy a well-behaved Poisson bracket,

$$
\left\{Q_{\Sigma}\left(\varkappa ; \phi^{u}\right), Q_{\Sigma}\left(\varkappa^{\prime} ; \phi^{u}\right)\right\}:=\boldsymbol{\Theta}_{\Sigma}^{u}\left(\varkappa^{v}, \varkappa^{\prime v}\right)=Q_{\Sigma}\left(\left[\varkappa, \varkappa^{\prime}\right] ; \phi^{u}\right) .
$$

As to the matter of the physical interpretation of dressed charges, and their observability, two cases occur. If $\boldsymbol{u}=\boldsymbol{u}(\varphi)$, the ansatz (3.26) implies its dressed version

$$
A^{\boldsymbol{u}}=A_{0}^{\boldsymbol{u}}+\alpha^{\boldsymbol{u}}\left(:=\left(\boldsymbol{u}^{-1} A_{0} \boldsymbol{u}+\boldsymbol{u}^{-1} d \boldsymbol{u}\right)+\left(\boldsymbol{u}^{-1} \alpha \boldsymbol{u}\right)\right),
$$

which can simply be plugged into (4.19), so that if we further declare the Killing equation

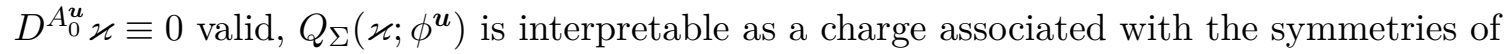
the background field $A_{0}^{u}$ conserved on-shell. It splits as a constant contribution from $A_{0}^{u}$, against which is measured/observed the contribution from $\alpha^{u}$ (and $\varphi^{u}$ ).

In case $\boldsymbol{u}=\boldsymbol{u}(A)$, special care must be taken as the dressing field is then itself affected by the affine ansatz (3.26). Splitting of the charge into background and physical contributions may be trickier.

None of this is available when using connections to realise the basic presymplectic structure of $L$, for which the ambiguity - or change of "coordinatisation" - is not in general captured/parametrised by a group $\mathcal{G}$.

Comments 1. In most of the literature on edge mode, the dressed presymplectic structure (4.12) goes by the name of extended presymplectic structure, as edge modes are seen as new degrees of freedom (d.o.f.) living at the boundary. The interpretation being that 
$\partial \Sigma$ breaks gauge invariance and that edge modes are kind of Goldstone bosons. Yet the DFM shows [33] that if a $\phi$-independent dressing field $u$ is introduced by fiat in a theory - as its interpretation as new d.o.f. implies - it means that the underlying bundle one works with is trivial and that one actually has $\mathcal{G} \simeq \mathcal{H}$, i.e. the 'ambiguity' symmetry $\mathcal{G}$ is just the original gauge symmetry $\mathcal{H}$ in another guise. This would be a challenge to the interpretation of $\mathcal{G}$ as a new symmetry stemming from the introduction of edge modes.

Another notion found in the edge mode literature that we are here bound to challenge, is the interpretation of $\mathcal{G}$ - and $\mathcal{G}$ - as a physical transformation group, usually referred to as surface or boundary symmetry, ${ }^{24}$ insofar as it is seen as a transformation group acting non-trivially on physical degrees of freedom. Seing that $\phi^{\boldsymbol{u}}$ are $\mathcal{H}$-invariant fields, thus potentially physical d.o.f., eq. (2.42) would indeed seem to suggest that $\mathcal{G}$ transforms physical field configurations, making it literally a physical symmetry. Yet as we have argued in section 2.3 and reminded above, $\mathcal{G}$ is the structure group of $\Phi^{u}$, whose base $\mathcal{M}^{u}$ is isomorphic to the physical configuration space $\mathcal{M}$ (the base of the $\mathcal{H}$-bundle $\Phi$ ). And as the SES (2.43) shows, $\mathcal{G}$ acts trivially on $\mathcal{M}^{u} \simeq \mathcal{M}$ and therefore cannot be a physical symmetry as understood in the edge mode literature. Physical symmetries, understood as acting nontrivially on physical d.o.f., rather belong to (subgroups of) $\operatorname{Diff}\left(\mathcal{M}^{u}\right) \simeq \operatorname{Diff}(\mathcal{M})$. This is not in contradiction with the above discussion on the physical interpretability of charges associated with $\mathcal{G}$. It simply means that the problem of the physical relevance of $\mathcal{G}$ as a symmetry group is of the same nature as that of the original gauge group $\mathcal{H}$, and does not enjoy a more immediate physical interpretation.

In the recent [69], edge modes are interpreted as a type of "dynamical reference frames", that is d.o.f. chosen to keep track of the evolution of all the others. In that case edge mode are not new d.o.f. added arbitrarily, but built from the field content of the theory which is in line with the philosophy using variational connections as advocated e.g. in [9]. So, they are indeed field-dependent dressing fields as described here: compare eq. (60) to our definition above eq. (2.45). In the case at hand, where one wants to keep track of the d.o.f. of a region of spacetime w.r.t. those of its complement, these "group-valued reference frames" are built explicitly as Wilson lines. Such examples of non-local fielddependent dressing fields is often encountered, Dirac dressings being the prototype [70, 71]. We elaborate on the relevance of the existence of local $v s$ non-local dressing fields in our next comment.

Comments 2. Regarding the aim of building a basic symplectic structure associated with a theory $L$, the existence of $\mathcal{G}$ as a symmetry of the dressed theory $L^{u}$ spoils everything: the boundary problem posed by the $\mathcal{H}$-symmetry of $L$ as been solved, but as is clear from (4.17), a boundary problem reemerges w.r.t. $\mathcal{G}$. This reflects the two options we had advertised (below (2.44)): either 1) the constructive procedure of building a dressing field $\boldsymbol{u}$ from the field content $\phi$ is free (enough) of ambiguity so that $\mathcal{G}$ is 'small', perhaps reduced to a rigid or discret group, in which case the boundary problem can be considered solved. Or 2) $\mathcal{G}$ is indeed a new meaningful gauge symmetry giving rise to its own boundary problem, but whose physical relevance may manifests through charges.

\footnotetext{
${ }^{24}$ Again, because $\boldsymbol{u}$ is usually seen as living on $\partial \Sigma$ when introduced to restore the invariance (horizontality) of on-shell quantities, so that $\xi \in \mathcal{G}$ is seen as a map $\xi: \partial \Sigma \rightarrow G$.
} 
Yet even in the first case an important remark should be raised. It the constructive procedure ends-up producing a dressing field $\boldsymbol{u}$ which is local (in the sense of field theory), then the theory can be rewritten so that each individual field variable $\phi^{\boldsymbol{u}}$ is a gauge singlet yet still remains local. If the gauge symmetry of a theory can be thus eliminated without losing locality, it is said to be an artificial gauge symmetry [72] (the terminology "fake symmetry" of Jackiw \& $\mathrm{Pi}$ [73] covers the same notion). On the contrary, if it happens that no dressing field can be found, or that only non-local ones can be produced, then the dressed variables $\phi^{u}$ are $\mathcal{H}$-invariant but non-local. In such theories, the gauge symmetry is only eliminated at the cost of locality, and this is usually recognised as hallmark and physical signature of substantial gauge symmetries. This distinction between two classes of gauge symmetries, one physically relevant the other not, generalises the well-know distinction between artificial and substantive general covariance familiar in the foundation of general relativistic physics [72]. The DFM methods can then be used as a tool to assess the nature of the gauge symmetry of a theory [55, 74].

Arguably there is no genuine boundary problem in a theory with an artificial gauge symmetry, or if there is, the dressed presymplectic structure (4.12) solves it in case 1). A genuine boundary problem arises only for substantial gauge symmetries - be it the original $\mathcal{H}$ or the new $\mathcal{G}$, case 2 ) - and the fact that it is only solved by sacrificing the locality of the theory may be seen as yet another signal of the non-local — or non-separable character of the physics described by (true) gauge field theories [31, 75-79].

Let us know consider immediate applications of the last two sections to Yang-Mills theory and 4D gauge gravity, which will illustrate in particular the above discussion.

\subsection{Applications}

\subsubsection{Yang-Mills theory}

We here start from, and rely on, the results of section 3.3.1. We have $H=\mathrm{SU}(n), \mathcal{H}=$ $\mathcal{S U}(n)=\mathcal{G}$.

Basic with connections. As the Lagrangian of the theory is basic, $L_{\mathrm{YM}} \in \Omega_{\text {basic }}^{0}(\Phi)$, the field equations and the presymplectic structure are invariant, $\boldsymbol{E}_{\mathrm{YM}}, \boldsymbol{\theta}_{\Sigma}^{\mathrm{YM}}, \boldsymbol{\Theta}_{\Sigma}^{\mathrm{YM}} \in \Omega_{\mathrm{inv}}^{\bullet}(\Phi)$. Therefore, given a connection $\boldsymbol{\omega} \in \Omega_{\mathrm{eq}}^{1}(\Phi, \mathrm{Lie} \mathcal{H})$, one can proceed and write down the corresponding basic versions.

By application of (4.2) and given (3.31), we get the basic field equations

$$
\begin{aligned}
\boldsymbol{E}_{\boldsymbol{\omega}}^{\mathrm{YM}} & =\boldsymbol{E}_{\mathrm{YM}}-d E_{\mathrm{YM}}(\boldsymbol{\omega} ; \phi), \\
& =\boldsymbol{E}^{\mathrm{YM}}-d \operatorname{Tr}(\boldsymbol{\omega}\{D * F-J\}), \quad \in \Omega_{\text {basic }}^{1}(\Phi) .
\end{aligned}
$$

By (4.7) and given (3.31)-(3.32), we obtain immediately the basic presymplectic structure,

$$
\begin{aligned}
\boldsymbol{\theta}_{\boldsymbol{\omega}, \Sigma}^{\mathrm{YM}} & =\boldsymbol{\theta}_{\Sigma}-\int_{\partial \Sigma} \theta_{\mathrm{YM}}(\boldsymbol{\omega} ; \phi)+\int_{\Sigma} E_{\mathrm{YM}}(\boldsymbol{\omega} ; \phi) \quad \in \Omega_{\text {basic }}^{1}(\Phi), \\
& =\boldsymbol{\theta}_{\Sigma}^{\mathrm{YM}}-\int_{\partial \Sigma} \operatorname{Tr}(\boldsymbol{\omega} * F)+\int_{\Sigma} \operatorname{Tr}(\boldsymbol{\omega}\{D * F-J\}),
\end{aligned}
$$




$$
\begin{aligned}
\boldsymbol{\Theta}_{\boldsymbol{\omega}, \Sigma}^{\mathrm{YM}} & =\boldsymbol{\Theta}_{\Sigma}-\int_{\partial \Sigma} \boldsymbol{d} \theta_{\mathrm{YM}}(\boldsymbol{\omega} ; \phi)+\int_{\Sigma} \boldsymbol{d} E_{\mathrm{YM}}(\boldsymbol{\omega} ; \phi) \quad \in \Omega_{\mathrm{basic}}^{2}(\Phi), \\
& =\boldsymbol{\Theta}_{\Sigma}^{\mathrm{YM}}-\int_{\partial \Sigma} \boldsymbol{d} \operatorname{Tr}(\boldsymbol{\omega} * F)+\int_{\Sigma} \boldsymbol{d} \operatorname{Tr}(\boldsymbol{\omega}\{D * F-J\}),
\end{aligned}
$$

This reproduces eq. (6.28)-(6.33) of [8] (who consider coupling to spinors rather than to scalar fields, which changes nothing of substance). Eq. (4.25)-(4.26) would be one coordinatisation of the phase space of YM theory over $\Sigma,\left(\mathcal{M}_{\mathcal{S}}, \Theta_{\Sigma}^{b}\right)_{\mathrm{YM}}$. Others are obtained under change of connection $\boldsymbol{\omega}^{\prime}=\boldsymbol{\omega}+\boldsymbol{\beta}$, as by (4.9), (4.8) and (4.10) we have:

$$
\begin{aligned}
\boldsymbol{E}_{\boldsymbol{\omega}^{\prime}}^{\mathrm{YM}} & =\boldsymbol{E}_{\boldsymbol{\omega}}^{\mathrm{YM}}-d \operatorname{Tr}(\boldsymbol{\beta}\{D * F-J\}), \\
\boldsymbol{\theta}_{\boldsymbol{\omega}^{\prime}, \Sigma}^{\mathrm{YM}} & =\boldsymbol{\theta}_{\boldsymbol{\omega}, \Sigma}^{\mathrm{YM}}-\int_{\partial \Sigma} \operatorname{Tr}(\boldsymbol{\beta} * F)+\int_{\Sigma} \operatorname{Tr}(\boldsymbol{\beta}\{D * F-J\}), \\
\boldsymbol{\Theta}_{\boldsymbol{\omega}^{\prime}, \Sigma}^{\mathrm{YM}} & =\boldsymbol{\Theta}_{\boldsymbol{\omega}, \Sigma}^{\mathrm{YM}}-\int_{\partial \Sigma} \boldsymbol{d} \operatorname{Tr}(\boldsymbol{\beta} * F)+\int_{\Sigma} \boldsymbol{d} \operatorname{Tr}(\boldsymbol{\beta}\{D * F-J\}) .
\end{aligned}
$$

As we commented at the end of section 4.1, these can also be seen a gluing relations of a sort between the basic objects constructed by two observers on each side of a region partitioned in two subregions by a boundary $\partial \Sigma$. Thus interpreted, (4.27) - the second line in particular - reproduces (on-shell) the results of section 6.7 of [9] and section 5.3 of [80] (see in particular eq. (83), in the free abelian case).

Let us remark that the relation between $\boldsymbol{D}^{\omega} \boldsymbol{\theta}_{\Sigma}^{\mathrm{YM}}$ and $\boldsymbol{\Theta}_{\omega, \Sigma}^{\mathrm{YM}}$, both basic 2-forms, is immediately read-off $(4.6)$ - itself a special case of (2.27) - to be:

$$
\begin{aligned}
\boldsymbol{D}^{\omega} \boldsymbol{\theta}_{\Sigma}^{\mathrm{YM}} & =\boldsymbol{\Theta}_{\boldsymbol{\omega}, \Sigma}^{\mathrm{YM}}+\int_{\partial \Sigma} \theta_{\mathrm{YM}}(\boldsymbol{\Omega} ; \phi)-\int_{\Sigma} E_{\mathrm{YM}}(\boldsymbol{\Omega} ; \phi), \\
& =\boldsymbol{\Theta}_{\boldsymbol{\omega}, \Sigma}^{\mathrm{YM}}+\int_{\partial \Sigma} \operatorname{Tr}(\boldsymbol{\Omega} * F)-\int_{\Sigma} \operatorname{Tr}(\boldsymbol{\Omega}\{D * F-J\}),
\end{aligned}
$$

with $\boldsymbol{\Omega} \in \Omega_{\text {tens }}^{2}(\Phi$, Lie $\mathcal{H})$ the curvature of $\boldsymbol{\omega}$. This reproduces (on-shell) the corollary 3.2 and the equation in section 3.4 of [9] (also found in footnote 27 of [13]), see also eq. (6.31)(6.32) of [8].

Basic with dressing fields. As we know by now, the existence of a $\phi$-dependent dressing field $\boldsymbol{u}: \Phi \rightarrow \mathcal{D} r[H, H]$ induces the existence of a flat connection $\stackrel{\circ}{\boldsymbol{\omega}}:=-\boldsymbol{d} \boldsymbol{u} \boldsymbol{u}^{-1}$, and another choice of dressing field $\boldsymbol{u}^{\prime}=\boldsymbol{u} \boldsymbol{\xi}$ induces $\stackrel{\circ}{\omega}^{\prime}=\stackrel{\circ}{\omega}+\stackrel{\circ}{\boldsymbol{\beta}}$. So, we could simply say that

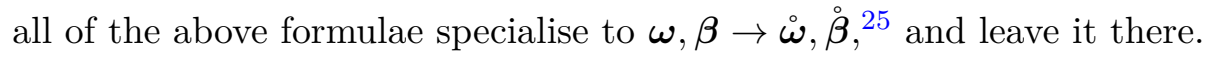

But there is of course more to say. By (4.11)-(4.12) and given (3.31)-(3.32), ${ }^{26}$ we have

$$
\begin{aligned}
\boldsymbol{E}_{\mathrm{YM}}^{\boldsymbol{u}} & =\boldsymbol{E}_{\mathrm{YM}}+d E_{\mathrm{YM}}\left(\boldsymbol{d} \boldsymbol{u} \boldsymbol{u}^{-1} ; \phi\right) \quad \in \Omega_{\mathrm{basic}}^{1}(\Phi), \\
& =\boldsymbol{E}_{\mathrm{YM}}+d \operatorname{Tr}\left(\boldsymbol{d} \boldsymbol{u} \boldsymbol{u}^{-1}\{D * F-J\}\right), \\
\left(\boldsymbol{\theta}_{\Sigma}^{\mathrm{YM}}\right)^{\boldsymbol{u}} & =\boldsymbol{\theta}_{\Sigma}^{\mathrm{YM}}+\int_{\partial \Sigma} \theta_{\mathrm{YM}}\left(\boldsymbol{d} \boldsymbol{u} \boldsymbol{u}^{-1} ; \phi\right)-\int_{\Sigma} E_{\mathrm{YM}}\left(\boldsymbol{d} \boldsymbol{u} \boldsymbol{u}^{-1} ; \phi\right) \quad \in \Omega_{\mathrm{basic}}^{1}(\Phi), \\
& =\boldsymbol{\theta}_{\Sigma}^{\mathrm{YM}}+\int_{\partial \Sigma} \operatorname{Tr}\left(\boldsymbol{d} \boldsymbol{u} \boldsymbol{u}^{-1} * F\right)-\int_{\Sigma} \operatorname{Tr}\left(\boldsymbol{d} \boldsymbol{u} \boldsymbol{u}^{-1}\{D * F-J\}\right),
\end{aligned}
$$

\footnotetext{
${ }^{25}$ With in particular the remark that (4.28) specialises to $\boldsymbol{D}^{\dot{\omega}} \boldsymbol{\theta}_{\Sigma}^{\mathrm{YM}}=\boldsymbol{\Theta}_{\dot{\omega}, \Sigma}^{\mathrm{YM}}$, i.e. the dressed presymplectic 2 -form (right) coincides with the $\stackrel{\circ}{\omega}$-covariant derivative of the original potential $\boldsymbol{\theta}_{\Sigma}^{\mathrm{YM}}$.

${ }^{26}$ Or using (3.43) and (3.44)-(3.45) together with the rule of thumb $\boldsymbol{\gamma} \rightarrow \boldsymbol{u}$.
} 


$$
\begin{aligned}
\left(\boldsymbol{\Theta}_{\Sigma}^{\mathrm{YM}}\right)^{\boldsymbol{u}} & =\boldsymbol{\Theta}_{\Sigma}^{\mathrm{YM}}+\int_{\partial \Sigma} \boldsymbol{d} \theta_{\mathrm{YM}}\left(\boldsymbol{d} \boldsymbol{u} \boldsymbol{u}^{-1} ; \phi\right)-\int_{\Sigma} \boldsymbol{d} E_{\mathrm{YM}}\left(\boldsymbol{d} \boldsymbol{u} \boldsymbol{u}^{-1} ; \phi\right) \quad \in \Omega_{\mathrm{basic}}^{2}(\Phi), \\
& =\boldsymbol{\Theta}_{\Sigma}^{\mathrm{YM}}+\int_{\partial \Sigma} \boldsymbol{d} \operatorname{Tr}\left(\boldsymbol{d} \boldsymbol{u} \boldsymbol{u}^{-1} * F\right)-\int_{\Sigma} \boldsymbol{d} \operatorname{Tr}\left(\boldsymbol{d} \boldsymbol{u} \boldsymbol{u}^{-1}\{D * F-J\}\right) .
\end{aligned}
$$

Observe again how only the Lie $\mathcal{H}$-linear part of $\boldsymbol{E}$ (and $\boldsymbol{\theta}$ ) contributes here, so that the field equations for the matter fields are irrelevant. Eq. (4.30)-(4.31) generalise, on-shell, eq. (2.19) and eq. (2.22)-(2.23) in [5]. As observed in section 4.2, these are the field equations and presymplectic structure of the dressed Lagrangian,

$$
L_{\mathrm{YM}}^{\boldsymbol{u}}=\mathrm{F}_{\boldsymbol{u}}^{\star} L_{\mathrm{YM}} \quad \text { i.e. } \quad L_{\mathrm{YM}}^{\boldsymbol{u}}(\phi)=L_{\mathrm{YM}}\left(\phi^{\boldsymbol{u}}\right)=\frac{1}{2} \operatorname{Tr}\left(F^{\boldsymbol{u}} * F^{\boldsymbol{u}}\right)+\frac{1}{2}\left\langle D^{\boldsymbol{u}} \varphi^{\boldsymbol{u}}, * D^{\boldsymbol{u}} \varphi^{\boldsymbol{u}}\right\rangle,
$$

seen as an invariant 0 -form on $\Phi^{u}$, and obtained via

$$
\boldsymbol{d} L_{\mathrm{YM}}^{\boldsymbol{u}}=\boldsymbol{E}_{\mathrm{YM}}^{\boldsymbol{u}}+d \boldsymbol{\theta}_{\mathrm{YM}}^{\boldsymbol{u}}=E_{\mathrm{YM}}\left(\boldsymbol{d} \phi^{\boldsymbol{u}} ; \phi^{\boldsymbol{u}}\right)+d \theta_{\mathrm{YM}}\left(\boldsymbol{d} \phi^{\boldsymbol{u}} ; \phi^{\boldsymbol{u}}\right) .
$$

Now, the a priori ambiguity in the choice/construction of a dressing is parametrised by a group $\mathcal{G}=\mathcal{S U}(n)$ (still) which is the structure group of $\Phi^{u}$ and a symmetry of $L_{\mathrm{YM}}^{u}$. We can thus associate to it Noether charges which, for $\varkappa \in \operatorname{Lie} \mathcal{G}$ inducing $\varkappa^{v} \in \Gamma\left(V \Phi^{u}\right)$, are immediately given by (4.19)

$$
\begin{aligned}
Q_{\Sigma}^{\mathrm{YM}}\left(\varkappa ; \phi^{\boldsymbol{u}}\right) & =\int_{\partial \Sigma} \theta_{\mathrm{YM}}\left(\varkappa ; \phi^{\boldsymbol{u}}\right)-\int_{\Sigma} E_{\mathrm{YM}}\left(\varkappa ; \phi^{\boldsymbol{u}}\right), \quad \text { s.t. } \quad \iota_{\varkappa}\left(\mathbf{\Theta}_{\Sigma}^{\mathrm{YM}}\right)^{\boldsymbol{u}}=-\boldsymbol{d} Q_{\Sigma}^{\mathrm{YM}}\left(\varkappa ; \phi^{\boldsymbol{u}}\right), \\
& =\int_{\partial \Sigma} \operatorname{Tr}\left(\varkappa * F^{\boldsymbol{u}}\right)-\int_{\Sigma} \operatorname{Tr}\left(\varkappa\left\{D^{\boldsymbol{u}} * F^{\boldsymbol{u}}-J^{\boldsymbol{u}}\right\}\right) .
\end{aligned}
$$

where $J^{u}=\left|* D^{\boldsymbol{u}} \varphi^{\boldsymbol{u}}\right\rangle\left\langle\varphi^{\boldsymbol{u}}|=| \rho(\boldsymbol{u})^{-1} * D \varphi\right\rangle\left\langle\rho(\boldsymbol{u})^{-1} \varphi\left|=u^{-1}\right| * D \varphi\right\rangle\langle\varphi| u=\boldsymbol{u}^{-1} J \boldsymbol{u}$. Quite naturally, the dressed 2-form $\left(\boldsymbol{\Theta}_{\Sigma}^{\mathrm{YM}}\right)^{\boldsymbol{u}}$ induces a Poisson bracket for these dressed charges which is by (4.20)

$$
\left\{Q_{\Sigma}^{\mathrm{YM}}\left(\varkappa ; \phi^{\boldsymbol{u}}\right), Q_{\Sigma}^{\mathrm{YM}}\left(\varkappa^{\prime} ; \phi^{\boldsymbol{u}}\right)\right\}:=\left(\boldsymbol{\Theta}_{\Sigma}^{\mathrm{YM}}\right) \boldsymbol{u}^{\boldsymbol{u}}\left(\varkappa^{v}, \varkappa^{\prime v}\right)=Q_{\Sigma}^{\mathrm{YM}}\left(\left[\varkappa, \varkappa^{\prime}\right] ; \phi^{\boldsymbol{u}}\right) .
$$

This generalises eq. (2.35)-(2.36)-(2.38) of [5]. Comparable formulae holds for fielddependent gauge parameters $\varkappa \in \operatorname{Lie} \mathcal{G}$, despite the fact that this time the dressed charges are non-integrable so that,

$$
\begin{aligned}
\iota_{\varkappa^{v}}\left(\boldsymbol{\Theta}_{\Sigma}^{\mathrm{YM}}\right)^{\boldsymbol{u}} & =-\boldsymbol{d} Q_{\Sigma}^{\mathrm{YM}}\left(\varkappa ; \phi^{\boldsymbol{u}}\right)+\boldsymbol{d} Q_{\Sigma}^{\mathrm{YM}}\left(\boldsymbol{d} \varkappa ; \phi^{\boldsymbol{u}}\right)=-\boldsymbol{d} Q_{\Sigma}^{\mathrm{YM}}\left(\varkappa ; \phi^{\boldsymbol{u}}\right), \\
& =-\int_{\partial \Sigma} \operatorname{Tr}\left(\varkappa * \boldsymbol{d} F^{\boldsymbol{u}}\right)+\int_{\Sigma} \operatorname{Tr}\left(\varkappa \boldsymbol{d}\left\{D^{\boldsymbol{u}} * F^{\boldsymbol{u}}-J^{\boldsymbol{u}}\right\}\right) .
\end{aligned}
$$

The dressed charge (4.34) could gain a clearer physical status by plugging the affine ansatz $A^{u}=A_{0}^{u}+\alpha^{u}$ and declaring $\varkappa$ a symmetry of the background $A_{0}^{u}$, so that similarly to (3.38) we get on-shell

$$
\begin{aligned}
Q_{\Sigma}^{\mathrm{YM}}\left(\varkappa ; \phi^{\boldsymbol{u}}\right)= & \int_{\partial \Sigma} \theta_{\mathrm{YM}}\left(\varkappa ; \phi^{\boldsymbol{u}}\right) \mid \mathcal{S} \\
& =\int_{\partial \Sigma} \operatorname{Tr}\left(\varkappa *\left\{F_{0}^{u}+f^{\boldsymbol{u}}+\frac{1}{2}\left[\alpha^{u}, \alpha^{u}\right]\right\}\right), \\
& =\int_{\partial \Sigma} \operatorname{Tr}\left(\varkappa * F_{0}^{u}\right)+\operatorname{Tr}\left(\varkappa * f^{\boldsymbol{u}}\right)=: Q_{\Sigma}^{\mathrm{YM}}\left(\varkappa ; A_{0}^{u}\right)+Q_{\Sigma}^{\mathrm{YM}}\left(\varkappa ; \alpha^{u}\right),
\end{aligned}
$$


with the second term interpreted as the contribution of the perturbation measured against the background. Of course, if the dressing field is built from the connection, it is itself affected by the affine ansatz (3.26) and special care must be applied as the charge may not be as simply written as in (4.37).

Seen as forms on the $\mathcal{G}$-bundle $\Phi^{u},(4.29)-(4.31)$ of course transform under its gauge group $\mathcal{G}$ by (4.17) as,

$$
\begin{aligned}
{\left[\boldsymbol{E}_{\mathrm{YM}}^{\boldsymbol{u}}\right]^{\boldsymbol{\xi}} } & =\boldsymbol{E}_{\mathrm{YM}}^{\boldsymbol{u}}+d \operatorname{Tr}\left(\boldsymbol{d} \boldsymbol{\xi} \boldsymbol{\xi}^{-1}\left\{D^{\boldsymbol{u}} * F^{\boldsymbol{u}}-J^{\boldsymbol{u}}\right\}\right), \\
& =\boldsymbol{E}_{\mathrm{YM}}^{\boldsymbol{u}}-d \operatorname{Tr}(\stackrel{\circ}{\boldsymbol{\beta}}\{D * F-J\}), \\
{\left[\left(\boldsymbol{\theta}_{\Sigma}^{\mathrm{YM}}\right)^{\boldsymbol{u}}\right]^{\boldsymbol{\xi}} } & =\left(\boldsymbol{\theta}_{\Sigma}^{\mathrm{YM}}\right)^{\boldsymbol{u}}+\int_{\partial \Sigma} \operatorname{Tr}\left(\boldsymbol{d} \boldsymbol{\xi} \boldsymbol{\xi}^{-1} * F^{\boldsymbol{u}}\right)-\int_{\Sigma} \operatorname{Tr}\left(\boldsymbol{d} \boldsymbol{\xi} \boldsymbol{\xi}^{-1}\left\{D^{\boldsymbol{u}} * F^{\boldsymbol{u}}-J^{\boldsymbol{u}}\right\}\right), \\
& =\left(\boldsymbol{\theta}_{\Sigma}^{\mathrm{YM}}\right)^{\boldsymbol{u}}-\int_{\partial \Sigma} \operatorname{Tr}(\stackrel{\circ}{\boldsymbol{\beta}} * F)+\int_{\Sigma} \operatorname{Tr}(\stackrel{\circ}{\boldsymbol{\beta}}\{D * F-J\}), \\
{\left[\left(\boldsymbol{\Theta}_{\Sigma}^{\mathrm{YM}}\right)^{\boldsymbol{u}}\right]^{\xi} } & =\left(\boldsymbol{\Theta}_{\Sigma}^{\mathrm{YM}}\right)^{\boldsymbol{u}}+\int_{\partial \Sigma} \boldsymbol{d} \operatorname{Tr}\left(\boldsymbol{d} \boldsymbol{\xi} \boldsymbol{\xi}^{-1} * F^{\boldsymbol{u}}\right)-\int_{\Sigma} \boldsymbol{d} \operatorname{Tr}\left(\boldsymbol{d} \boldsymbol{\xi} \boldsymbol{\xi}^{-1}\left\{D^{\boldsymbol{u}} * F^{\boldsymbol{u}}-J^{\boldsymbol{u}}\right\}\right), \\
& =\left(\boldsymbol{\Theta}_{\Sigma}^{\mathrm{YM}}\right)^{\boldsymbol{u}}-\int_{\partial \Sigma} \boldsymbol{d} \operatorname{Tr}(\stackrel{\circ}{\boldsymbol{\beta}} * F)+\int_{\Sigma} \boldsymbol{d} \operatorname{Tr}(\stackrel{\circ}{\boldsymbol{\beta}}\{D * F-J\}) .
\end{aligned}
$$

The display of each second line, where $\stackrel{\circ}{\boldsymbol{\beta}}=-\boldsymbol{u} \boldsymbol{d} \boldsymbol{\xi} \boldsymbol{\xi}^{-\mathbf{1}} \boldsymbol{u}^{-1}$, invites comparison with (4.27) and illustrates (4.15)-(4.16). These show that a boundary problem may reappear w.r.t. $\mathcal{G}$. Comments are thus in order.

Comments. We here have an occasion to illustrate the discussion held at the end of section 4.2 on the local vs non-local dressing fields in a model and the nature of its gauge symmetry.

In pure YM theory, there are no local dressing fields that can be built from the gauge potential (as far as is known). Only non-local dressings $\boldsymbol{u}=\boldsymbol{u}(A)$ seem possible, and basically related to holonomies of the connection. This is also true in YM theory coupled to spinors, as no local $\mathcal{S U}(n)$-dressing field can be constructed from a spinor field. In the special case of abelian YM theory coupled to spinors, i.e. spinorial electromagnetism (EM), the well-known Dirac phase [70] (see also [71] section 80) is an instance of non-local dressing field $\boldsymbol{u}=\boldsymbol{u}(A){ }^{27}$ In these cases, one would then conclude that the $\mathcal{S U}(n)$-gauge symmetry is substantial, as it is killed only at the cost of the locality of the theory.

In YM theory coupled to scalar fields, things are different. Considering for example the simple case of abelian YM theory coupled to a $\mathbb{C}$-scalar field, i.e. scalar electromagnetism, one can extract a local dressing field from the complex field by polar decomposition of the latter: $\varphi=\rho \boldsymbol{u}(\varphi) \in \mathbb{C}$, where $\rho=|\varphi| \in \mathbb{R}^{+}$and $\boldsymbol{u}(\varphi)=e^{i \theta} \in \mathrm{U}(1)$. Obviously the phase carries the gauge transformation, $\boldsymbol{u}(\varphi)^{\gamma}=\boldsymbol{u}\left(\varphi^{\gamma}\right)=\gamma^{-1} \boldsymbol{u}(\varphi)$. That is, the dressing field is simply the phase of the scalar field, which is local. Thus the invariant dressed field (2.40) $A^{\boldsymbol{u}}=A+\boldsymbol{u}^{-1} d \boldsymbol{u}$ and $\varphi^{\boldsymbol{u}}=\rho$ are both local and $\mathcal{U}(1)$-invariant. Any $\mathcal{U}(1)$-invariant Lagrangian $L(A, \varphi)$ can thus be dressed as in (4.32), giving a local theory with no gauge

\footnotetext{
${ }^{27}$ Dirac's eq. (16) and (21) in [70] — and eq. (110) in [71] — are abelian instances of the invariant dressed fields (2.40) above, and his non-local dressing field built from the gauge potential is given in eq. (18)-(19). Eq. (111) in [71] is the spinorial version of our $J^{u}$ after (4.34).
} 
symmetry. The $\mathcal{U}(1)$-symmetry of $L$ is then artificial. Furthermore, one may consider that the polar decomposition if rather unambiguous, so that no $\mathcal{G}$-transformations arise. This has noteworthy interpretive consequences for models couched in the framework of $\mathbb{C}$-scalar EM. Let us consider two: a semi-classical description of the Aharonov-Bohm (AB) effect and the abelian Higgs model.

The AB effect is usually seen as the prototypical phenomenon illustrating the nonlocality (or non-separability) inherent to gauge theories, as the phase shift in the interference pattern cannot be explained by the local interaction of electrons described by $\varphi$ with the gauge potential $A$, which are both non-invariant fields. ${ }^{28}$ Yet as we just saw, the theory can be rewritten so that electrons and the EM potential can be described by $\rho$ and $A^{u}$ respectively, which are invariant fields. Formulated within scalar EM, the AB effect is thus entirely non-problematic, as the phase shift can be explained via local interaction of invariant fields (as noted by philosopher D. Wallace [81]). Of course, in the more realistic framework of spinorial EM, since as stated above there is no extracting local dressings from spinors, the $\mathcal{U}(1)$-gauge symmetry is truly substantial, so the $\mathrm{AB}$ effect does actually highlights the non-local/non-separable character of EM phenomena.

The abelian Higgs model is often given as the simplest illustration of the notion of Spontaneous Symmetry Breaking (SSB): the gauge potential $A$ is minimally coupled to a $\mathbb{C}$-field $\varphi$ embedded in a potential $V(\varphi)=\mu^{2} \varphi^{*} \varphi+\lambda\left(\varphi^{*} \varphi\right)^{2}$ whose minima are $\varphi_{0}=0$ and $\left\{\varphi_{0}\right\}=\left\{\left|\varphi_{0}\right|=\sqrt{\frac{-\mu^{2}}{2 \lambda}}\right\}$. The first is unique and $\mathcal{U}(1)$-invariant, while the others form a $\mathcal{U}(1)$-orbit. The theory has two phases given by the sign of $-\mu^{2}$ : one in which the only vacuum solution is $\varphi_{0}=0, \mathcal{U}(1)$ preserved, and in which $A$ is massless. Another where the vacuum is degenerate, so that upon spontaneous selection of one point in $\left\{\varphi_{0}\right\}, \mathcal{U}(1)$ is broken and $A$ gains a mass proportional to $\sqrt{\frac{-\mu^{2}}{2 \lambda}}$.

But, upon dressing, the Lagrangian is rewritten as in (4.32) with the field $A^{u}$ minimally coupled to the $\mathbb{R}^{+}$-field $\rho$ embedded in the potential $V(\rho)=\mu^{2} \rho^{2}+\lambda \rho^{4}$ with only two minima $\rho_{0}=0$ and $\rho_{0}=\sqrt{\frac{-\mu^{2}}{2 \lambda}}$. This theory has no $\mathcal{U}(1)$-symmetry, yet still two phases according to the sign of $-\mu^{2}$ : one in which $A^{u}$ is massless, one in which it has a mass proportional to $\sqrt{\frac{-\mu^{2}}{2 \lambda}}$. One can thus appreciate that the notion of SSB is not what does the heavy lifting in the mass acquisition mechanism. Rather, the true operative notion is that of a phase transition between two vacuum structures (which are non-degenerate), and this doesn't necessarily coincide with a symmetry breaking - as is clearly the case here since the artificial $\mathcal{U}(1)$-symmetry is eliminated, and the physical d.o.f. exhibited, in both phases of the theory.

Coming back to general YM theories coupled to scalar fields, the same considerations applies to the electroweak (EW) model, where a local $\mathcal{S U}(2)$-dressing field $\boldsymbol{u}=\boldsymbol{u}(\varphi)$ is extracted from a polar decomposition of the $\mathbb{C}^{2}$-scalar field $\varphi$, coupled minimally to the $\mathrm{U}(1) \times \mathrm{SU}(2)$ gauge potential $A=a+B$. One may conclude that $\mathcal{S U}(2)$ is artificial and eliminate it, thereby exhibiting physical d.o.f, in both the massless and massive phases of the theory, leaving $\mathcal{U}(1)$ as the only substantial gauge symmetry of the model. SSB is thus

\footnotetext{
${ }^{28}$ Curiously, emphasis is often on the non-invariance of the gauge potential only, as if the wave function of the electron was unproblematic regarding gauge invariance.
} 
bypassed and the EW vacuum phase transition shown to be the operative phenomenon. For technical details on the DFM treatment of the EW model, and discussions on attending philosophical issues, see [54, 55], and [74] (to appear) for the inclusion of chiral fermions. The polar decomposition of $\varphi \in \mathbb{C}^{2}$ may be seen as suffering from some ambiguity, giving rise to residual $\mathcal{G}$-transformations, but [82] gives arguments as to why this ambiguity might be reduced to a discret choice.

Thus challenging the SSB interpretation of the EW model may seem an heretical thing to do. But it turns out that gauge-invariant treatments of Yang-Mill-Higgs models have a long history, starting very early on with Higgs and Kibble themselves in 1966 and 1967 (before the papers by Weinberg and Salam): one can easily see that eq. (23) in [83] and eq. (66) [84] are instances of (4.32). Before the conclusion of his paper, Kibble explicitly says "It is perfectly possible to describe [the theory] without ever introducing the notion of symmetry breaking". After the advent of the EW model, invariant treatments independently emerged several times, e.g. in [85] (compare eq. (75) and (77) to (2.40) and (4.32)) or in [86] (compare eq. (6.1) to (2.40)), and more recently [82, 87-90]. The review [91] provides an extensive list of references on recent developments in particle physics looking at such gaugeinvariant accounts of electroweak physics. Philosopher of physics have also seize the subject in the past fifteen years [92-97], pointing to the fact that a better understanding of the electroweak model, and gauge symmetries, might be necessary to make genuine progress beyond the current best established theories [74].

In any event, (4.29)-(4.31) give the dressed presymplectic structure of $\mathbb{C}$-EM and $\mathbb{C}^{2}$ YM theory, stemming from (4.32)-(4.33). From these are read-off the on-shell dressed presymplectic structure of both the abelian Higgs and EW models since, as we observed at the end of 3.3.1, the potential term does not contribute.

\subsubsection{D gauge gravity}

We now turn to a last illustration of the general framework: gravity. We will rely on the results of section 3.3.2, where $H=\mathrm{SO}(1,3)$ and $\mathcal{H}=\mathcal{S O}(1,3)$. Espousing the same template as the previous section, we first give a very swift description of the basic structure that would be obtained via variational connections, then engage in a more intricate discussion on what can - or could — be done via the DFM.

Basic with connections. The Lagrangian of the theory is basic, $L_{\mathrm{GR}} \in \Omega_{\mathrm{basic}}^{0}(\Phi)$, so the field equations and the presymplectic structure are invariant, $\boldsymbol{E}_{\mathrm{GR}}, \boldsymbol{\theta}_{\Sigma}^{\mathrm{GR}}, \boldsymbol{\Theta}_{\Sigma}^{\mathrm{GR}} \in \Omega_{\mathrm{inv}}^{\bullet}(\Phi)$. Then, given a connection $\boldsymbol{\omega} \in \Omega_{\mathrm{eq}}^{1}(\Phi$, Lie $\mathcal{H})$, one can write their basic counterparts. By application of (4.2) and given (3.54), we get the basic field equations

$$
\begin{aligned}
\boldsymbol{E}_{\omega}^{\mathrm{GR}} & =\boldsymbol{E}_{\mathrm{GR}}-d E_{\mathrm{GR}}(\boldsymbol{\omega} ; \phi), \\
& =\boldsymbol{E}_{\mathrm{GR}}+d\left\{\frac{2 \varepsilon}{\ell^{2}} \boldsymbol{\omega} \bullet T \wedge e^{T}-\operatorname{Tr}\left(\left|\rho_{*}(\boldsymbol{\omega}) \psi\right\rangle\langle * \gamma \psi|\right)\right\}, \quad \in \Omega_{\mathrm{basic}}^{1}(\Phi) .
\end{aligned}
$$


By (4.7) and given (3.54)-(3.55), the basic presymplectic structure is immediately found to be

$$
\begin{aligned}
\boldsymbol{\theta}_{\boldsymbol{\omega}, \Sigma}^{\mathrm{GR}} & =\boldsymbol{\theta}_{\Sigma}-\int_{\partial \Sigma} \theta_{\mathrm{GR}}(\boldsymbol{\omega} ; \phi)+\int_{\Sigma} E_{\mathrm{GR}}(\boldsymbol{\omega} ; \phi) \quad \in \Omega_{\mathrm{basic}}^{1}(\Phi), \\
& =\boldsymbol{\theta}_{\Sigma}^{\mathrm{GR}}-\int_{\partial \Sigma} \boldsymbol{\omega} \bullet F-\int_{\Sigma} \frac{2 \varepsilon}{\ell^{2}} \boldsymbol{\omega} \bullet T \wedge e^{T}-\operatorname{Tr}\left(\left|\rho_{*}(\boldsymbol{\omega}) \psi\right\rangle\langle * \gamma \psi|\right), \\
\boldsymbol{\Theta}_{\boldsymbol{\omega}, \Sigma}^{\mathrm{GR}} & =\boldsymbol{\Theta}_{\Sigma}-\int_{\partial \Sigma} \boldsymbol{d} \theta_{\mathrm{GR}}(\boldsymbol{\omega} ; \phi)+\int_{\Sigma} \boldsymbol{d} E_{\mathrm{GR}}(\boldsymbol{\omega} ; \phi) \quad \in \Omega_{\mathrm{basic}}^{2}(\Phi), \\
& =\boldsymbol{\Theta}_{\Sigma}^{\mathrm{GR}}-\int_{\partial \Sigma} \boldsymbol{d}(\boldsymbol{\omega} \bullet F)-\int_{\Sigma} \frac{2 \varepsilon}{\ell^{2}} \boldsymbol{d}\left(\boldsymbol{\omega} \bullet T \wedge e^{T}\right)-\boldsymbol{d} \operatorname{Tr}\left(\left|\rho_{*}(\boldsymbol{\omega}) \psi\right\rangle\langle * \gamma \psi|\right),
\end{aligned}
$$

Eq. (4.40)-(4.41) would be one coordinatisation of the phase space of GR over $\Sigma$, $\left(\mathcal{M}_{\mathcal{S}}, \Theta_{\Sigma}^{b}\right)_{\mathrm{GR}}$. Others would of course be obtained under change of connection $\boldsymbol{\omega}^{\prime}=\boldsymbol{\omega}+\boldsymbol{\beta}$, as by (4.9), (4.8) and (4.10) we have:

$$
\begin{aligned}
\boldsymbol{E}_{\omega^{\prime}}^{\mathrm{GR}} & =\boldsymbol{E}_{\boldsymbol{\omega}}^{\mathrm{GR}}+d\left\{\frac{2 \varepsilon}{\ell^{2}} \boldsymbol{\beta} \bullet T \wedge e^{T}-\operatorname{Tr}\left(\left|\rho_{*}(\boldsymbol{\beta}) \psi\right\rangle\langle * \gamma \psi|\right)\right\}, \\
\boldsymbol{\theta}_{\omega^{\prime}, \Sigma}^{\mathrm{GR}} & =\boldsymbol{\theta}_{\boldsymbol{\omega}, \Sigma}^{\mathrm{GR}}-\int_{\partial \Sigma} \boldsymbol{\beta} \bullet F-\int_{\Sigma} \frac{2 \varepsilon}{\ell^{2}} \boldsymbol{\beta} \bullet T \wedge e^{T}-\operatorname{Tr}\left(\left|\rho_{*}(\boldsymbol{\beta}) \psi\right\rangle\langle * \gamma \psi|\right), \\
\boldsymbol{\Theta}_{\omega^{\prime}, \Sigma}^{\mathrm{GR}} & =\boldsymbol{\Theta}_{\boldsymbol{\omega}, \Sigma}^{\mathrm{GR}}-\int_{\partial \Sigma} \boldsymbol{d}(\boldsymbol{\beta} \bullet F)-\int_{\Sigma} \frac{2 \varepsilon}{\ell^{2}} \boldsymbol{d}\left(\boldsymbol{\beta} \bullet T \wedge e^{T}\right)-\boldsymbol{d} \operatorname{Tr}\left(\left|\rho_{*}(\boldsymbol{\beta}) \psi\right\rangle\langle * \gamma \psi|\right) .
\end{aligned}
$$

As per section 4.1, these can be seen a gluing relations the basic structures constructed by two observers on each side of a region partitioned in two subregions by a boundary $\partial \Sigma$.

Finally, let us notice the relation between the basic 2 -forms $\boldsymbol{D}^{\omega} \boldsymbol{\theta}_{\Sigma}^{\mathrm{GR}}$ and $\boldsymbol{\Theta}_{\omega, \Sigma}^{\mathrm{GR}}$, which by $(4.6)$ - or $(2.27)$ - is:

$$
\begin{aligned}
\boldsymbol{D}^{\omega} \boldsymbol{\theta}_{\Sigma}^{\mathrm{GR}} & =\boldsymbol{\Theta}_{\boldsymbol{\omega}, \Sigma}^{\mathrm{GR}}+\int_{\partial \Sigma} \theta_{\mathrm{GR}}(\boldsymbol{\Omega} ; \phi)-\int_{\Sigma} E_{\mathrm{GR}}(\boldsymbol{\Omega} ; \phi), \\
& =\boldsymbol{\Theta}_{\boldsymbol{\omega}, \Sigma}^{\mathrm{GR}}+\int_{\partial \Sigma} \boldsymbol{\Omega} \bullet F+\int_{\Sigma} \frac{2 \varepsilon}{\ell^{2}} \boldsymbol{\Omega} \bullet T \wedge e^{T}-\operatorname{Tr}\left(\left|\rho_{*}(\boldsymbol{\Omega}) \psi\right\rangle\langle * \gamma \psi|\right),
\end{aligned}
$$

with $\boldsymbol{\Omega} \in \Omega_{\text {tens }}^{2}(\Phi$, Lie $\mathcal{H})$ the curvature of $\boldsymbol{\omega}$. Both coincide when $\boldsymbol{\omega}=\stackrel{\circ}{\boldsymbol{\omega}}$ is flat, as would be the case if using the DFM was an option.

Basic with dressing fields. The use of the DFM in the gravitational case is slightly more subtle than in the YM context. It is instructive to address first the case of the pure gravitational theory, before coming back to the coupling to spinors.

Pure gauge gravity: let us quickly set the stage. The pure gravity sector is given by the McDowell-Mansouri term in (3.53), a basic 0-form on the $\mathcal{H}$-bundle space of Cartan connections $\Phi=\overline{\mathcal{A}}$ :

$$
\begin{aligned}
L_{\mathrm{MM}}(\bar{A}) & =\frac{1}{2} F \bullet F, \quad \in \Omega_{\mathrm{basic}}^{0}(\overline{\mathcal{A}}), \quad \text { so } \quad \boldsymbol{d} L_{\mathrm{MM}}=\boldsymbol{E}_{\mathrm{MM}}+d \boldsymbol{\theta}_{\mathrm{MM}} \quad \in \Omega_{\mathrm{basic}}^{1}(\overline{\mathcal{A}}) \text { with }, \\
\boldsymbol{E}_{\mathrm{MM}} & \left.=-\frac{2 \varepsilon}{\ell^{2}}\left\{\boldsymbol{d} A \bullet T \wedge e^{T}+\boldsymbol{d} e \wedge e^{T} \bullet F\right)\right\} \in \Omega_{\mathrm{inv}}^{1}(\overline{\mathcal{A}}) \text { so } \boldsymbol{\theta}_{\mathrm{MM}}=\boldsymbol{d} A \bullet F \in \Omega_{\mathrm{inv}}^{1}(\overline{\mathcal{A}}),
\end{aligned}
$$


which is also read-off (3.54)-(3.55). The presymplectic 2-form is $\Theta_{\Sigma}^{\mathrm{MM}}=-\int_{\Sigma} \boldsymbol{d} A \bullet \boldsymbol{d} F$. From (3.6), the Lorentz charges are

$$
Q_{\Sigma}^{\mathrm{MM}}(\chi ; \bar{A})=\int_{\partial \Sigma} \theta_{\mathrm{MM}}(\chi ; \bar{A})-\int_{\Sigma} E_{\mathrm{MM}}(\chi ; \bar{A})=\int_{\partial \Sigma} \chi \bullet F+\int_{\Sigma} \frac{2 \varepsilon}{\ell^{2}} \chi \bullet T \wedge e^{T},
$$

as is also seen from (3.56). In pure GR, the restriction on-shell is too strong, and imposing normality of $\bar{A}$, that is $T=0$, is enough to have the charge written as a pure boundary term. In full generality, $\Theta_{\Sigma}^{\mathrm{MM}}$ induces a Poisson bracket for the above charges $\left\{Q_{\Sigma}^{\mathrm{MM}}(\chi ; \bar{A}), Q_{\Sigma}^{\mathrm{MM}}(\eta ; \bar{A})\right\}:=\boldsymbol{\Theta}_{\Sigma}^{\mathrm{MM}}\left(\chi^{v}, \eta^{v}\right)=Q_{\Sigma}^{\mathrm{MM}}([\chi, \eta] ; \bar{A})$. The $\mathcal{H}=\mathcal{S O}(1,3)$ fielddependent gauge transformations of $E_{\mathrm{MM}}, \boldsymbol{\theta}_{\Sigma}^{\mathrm{MM}}$ and $\boldsymbol{\Theta}_{\Sigma}^{\mathrm{MM}}$ are of course found to be special cases of (3.62)-(3.64) (without $\psi$-terms).

We may notice that a local $\mathrm{SO}(1,3)$-dressing field is readily available in gravity. Indeed we have that the soldering part of the Cartan connection $\bar{A}$ transforms as $R_{\gamma} e:=e^{\gamma}=$ $\gamma^{-1} e$. Given a coordinate system $\left\{x^{\mu}\right\}$ on $U \subset M$, the soldering is $e^{a}=e^{a}{ }_{\mu} d x^{\mu}$, or $e=\boldsymbol{e} \cdot d x$, so the map $\boldsymbol{e}:=e^{a}{ }_{\mu}: U \rightarrow \mathrm{GL}(4)$ is s.t. $\boldsymbol{e}^{\gamma}=\gamma^{-1} \boldsymbol{e}$. The tetrad is thus a field-dependent local Lorentz dressing field $\boldsymbol{u}: \overline{\mathcal{A}} \rightarrow \operatorname{Dr}[\mathrm{SO}, G L], \bar{A} \mapsto \boldsymbol{u}(\bar{A})=\boldsymbol{e}$, s.t. $R_{\gamma}^{\star} \boldsymbol{u}(\bar{A})=\boldsymbol{u}\left(R_{\gamma} \bar{A}\right)=\boldsymbol{u}\left(\bar{A}^{\gamma}\right)=\boldsymbol{e}^{\gamma}=\gamma^{-1} \boldsymbol{e}=\gamma^{-1} \boldsymbol{u}(\bar{A})$.

Using the Lorentz dressing $\boldsymbol{u}(\bar{A})=\boldsymbol{e}$, written as the $5 \times 5$ matrix $\overline{\boldsymbol{u}}=\left(\begin{array}{ll}\boldsymbol{u} & 0 \\ 0 & 1\end{array}\right)$, the $\mathcal{S O}$ invariant dressed Cartan connection is

$$
\begin{aligned}
\bar{A}^{\overline{\boldsymbol{u}}}=\overline{\boldsymbol{u}}^{-1} \bar{A} \overline{\boldsymbol{u}}+\overline{\boldsymbol{u}}^{-1} d \overline{\boldsymbol{u}}= & \left(\begin{array}{cc}
A^{\boldsymbol{u}} & \frac{1}{\ell} \boldsymbol{e}^{\boldsymbol{u}} \\
\frac{-\varepsilon}{\ell}\left(e^{t}\right)^{\boldsymbol{u}} & 0
\end{array}\right) \\
& =\left(\begin{array}{cc}
\boldsymbol{e}^{-1} A \boldsymbol{e}+\boldsymbol{e}^{-1} d \boldsymbol{e} & \frac{1}{\ell} d x \\
\frac{-\varepsilon}{\ell} d x^{T} \cdot \boldsymbol{g} & 0
\end{array}\right)=:\left(\begin{array}{cc}
\Gamma & \frac{1}{\ell} d x \\
\frac{-\varepsilon}{\ell} d x^{T} \cdot \boldsymbol{g} & 0
\end{array}\right)=\bar{\Gamma} .
\end{aligned}
$$

where $\Gamma=\Gamma_{\nu}^{\mu}=\Gamma_{\nu, \rho}^{\mu} d x^{\rho}$ is the familiar linear connection with values in $M(4, \mathbb{R})=$ LieGL(4), $d x=\delta^{\mu}{ }_{\rho} d x^{\rho}$ and $d x^{T} \cdot \boldsymbol{g}=d x^{\mu} g_{\mu \nu}$. The metricity condition is automatic, as we have $\nabla \boldsymbol{g}:=d \boldsymbol{g}-\Gamma^{T} \boldsymbol{g}-\boldsymbol{g} \Gamma=-\boldsymbol{e}^{T}\left(A^{T} \eta+\eta A\right) \boldsymbol{e}=0$. A similar matrix computation for the dressed Cartan curvature gives,

$$
\bar{F}^{\boldsymbol{u}}=\overline{\boldsymbol{u}}^{-1} \bar{F} \overline{\boldsymbol{u}} \Rightarrow\left\{\begin{array}{l}
F^{\boldsymbol{u}}=\boldsymbol{e}^{-1} F \boldsymbol{e}=: \mathrm{F}=\mathrm{R}-\frac{\varepsilon}{\ell^{2}} d x \wedge d x^{T} \cdot \boldsymbol{g}, \\
T^{u}=e^{-1} T=: \mathrm{T}=\Gamma \wedge d x,
\end{array}\right.
$$

where $\mathrm{R}=d \Gamma+\frac{1}{2}[\Gamma, \Gamma]=\frac{1}{2} \mathrm{R}^{\mu}{ }_{\nu, \rho \sigma} d x^{\rho} \wedge d x^{\sigma}$ is $M(4, \mathbb{R}$ )-valued (with components the usual Riemann tensor), and $\mathbf{T}=\mathbf{T}^{\mu}=\frac{1}{2} \mathbf{T}^{\mu}{ }_{\rho \sigma} d x^{\rho} \wedge d x^{\sigma}=\Gamma_{\rho \sigma}^{\mu} d x^{\rho} \wedge d x^{\sigma}$ is the known expression for the torsion.

Yet another simple matrix computation shows that, by (2.49),

$$
\begin{aligned}
\boldsymbol{d} \bar{A}^{\overline{\boldsymbol{u}}} & =\overline{\boldsymbol{u}}^{-1}\left(\boldsymbol{d} \bar{A}+D^{\bar{A}}\left\{\boldsymbol{d} \overline{\boldsymbol{u}} \overline{\boldsymbol{u}}^{-1}\right\}\right) \overline{\boldsymbol{u}} \\
& =\left(\begin{array}{cc}
\boldsymbol{d} A^{\boldsymbol{u}} & \frac{1}{\ell} \boldsymbol{d} e^{\boldsymbol{u}} \\
\frac{-\varepsilon}{\ell} \boldsymbol{d}\left(e^{t}\right)^{\boldsymbol{u}} & 0
\end{array}\right) \\
& =\left(\begin{array}{cr}
\boldsymbol{e}^{-1}\left(\boldsymbol{d} A+D^{A}\left\{\boldsymbol{d e} \boldsymbol{e}^{-1}\right\}\right) & 0 \\
\frac{-\varepsilon}{\ell}\left(\boldsymbol{d} e^{T} \eta \boldsymbol{e}+e^{T} \eta \boldsymbol{d e}\right) & 0
\end{array}\right)=:\left(\begin{array}{rr}
\boldsymbol{d} \Gamma & 0 \\
\frac{-\varepsilon}{\ell} d x^{t} \cdot \boldsymbol{d} \boldsymbol{g} & 0
\end{array}\right)=\boldsymbol{d} \bar{\Gamma} .
\end{aligned}
$$


where one uses that $\boldsymbol{d} \boldsymbol{e} e^{-1} e=\boldsymbol{d} e^{a}{ }_{\mu}\left(e^{-1}\right)^{\mu}{ }_{b} e=\boldsymbol{d} e^{a}{ }_{\mu} d x^{\mu}=\boldsymbol{d} e$ to have the top right component vanish (as also heuristically expected from (4.46) and $\boldsymbol{d} d x=0$ ).

As the dressing field takes values in a group larger than the structure group $\mathrm{SO}(1,3)$, to apply our general results we need to clarify the following technical point: the polynomial (3.50) we used to write the pure gravity sector of the Lagrangian for $4 \mathrm{D}$ gravity in sections 3.3.2 is SO-invariant by (3.51). It is the restriction of the $G L$-invariant polynomial $\bar{P}: \otimes^{k} M(2 k, \mathbb{R}) \rightarrow \mathbb{R}$ given by

$$
\begin{aligned}
\bar{P}\left(M_{1}, \ldots, M_{k}\right) & =\sqrt{|\operatorname{det}(\boldsymbol{g})|} M_{1} \bullet \ldots \bullet M_{k} \\
& :=\sqrt{|\operatorname{det}(\boldsymbol{g})|} M_{1}^{\mu_{1} \mu_{2}} M_{2}^{\mu_{3} \mu_{4}} \ldots M_{k}^{\mu_{2 k-1} \mu_{2 k}} \varepsilon_{\mu_{1} \ldots \mu_{2 k}},
\end{aligned}
$$

The $G L$-invariance under the substitution $\boldsymbol{g} \rightarrow G^{T} \boldsymbol{g} G$ and $M \rightarrow G^{-1} M G^{-1 T}$, with $G=$ $G^{\alpha}{ }_{\beta} \in \mathrm{GL}(4)$, is easily checked (by a computation analogue to (3.51)). One obtains the SOinvariant polynomial $P$ by the substitution $\boldsymbol{g} \rightarrow \eta$. Conversely, if in $P$ one plugs variables $\boldsymbol{e} M \boldsymbol{e}^{-1} \eta^{-1}=\boldsymbol{e} \boldsymbol{M} \boldsymbol{g}^{-1} \boldsymbol{e}^{T}$ (restoring on the left $\eta^{-1}$ that was kept tacit) then by (3.51) again we get

$$
\begin{aligned}
P\left(\boldsymbol{e} M_{1} \boldsymbol{e}^{-1} \eta^{-1}, \ldots, \boldsymbol{e} M_{k} \boldsymbol{e}^{-1} \eta^{-1}\right) & =\boldsymbol{e} M_{1} \boldsymbol{g}^{-1} \boldsymbol{e}^{T} \bullet \ldots \bullet \boldsymbol{e} M_{k} \boldsymbol{g}^{-1} \boldsymbol{e}^{T} \\
& =\operatorname{det}(\boldsymbol{e}) M_{1} \boldsymbol{g}^{-1} \bullet \ldots \bullet M_{k} \boldsymbol{g}^{-1} \\
& =\bar{P}\left(M_{1} \boldsymbol{g}^{-1}, \ldots, M_{k} \boldsymbol{g}^{-1}\right)
\end{aligned}
$$

To lighten the notation, we will omit $\boldsymbol{g}^{-1}$ in front of variables in expressions involving $\bar{P}$, as it should be clear from the context that indices must be raised.

Now, the dressed field equations and presymplectic structure associated with $L_{\mathrm{MM}}$ are, by $(4.11)-(4.12)$,

$$
\begin{aligned}
& \boldsymbol{E}_{\mathrm{MM}}^{\boldsymbol{u}}=E_{\mathrm{MM}}+d E\left(\boldsymbol{d u} \boldsymbol{u}^{-1} ; \bar{A}\right)=E_{\mathrm{MM}}-\frac{2 \varepsilon}{\ell^{2}} d\left(\boldsymbol{d e} \boldsymbol{e}^{-1} \bullet T \wedge e^{T}\right), \\
& \left(\boldsymbol{\theta}_{\Sigma}^{\mathrm{MM}}\right)^{\boldsymbol{u}}=\boldsymbol{\theta}_{\Sigma}^{\mathrm{MM}}+\int_{\partial \Sigma} \theta\left(\boldsymbol{d} \boldsymbol{u} \boldsymbol{u}^{-1} ; \bar{A}\right)-\int_{\Sigma} E\left(\boldsymbol{d} \boldsymbol{u} \boldsymbol{u}^{-1} ; \bar{A}\right), \\
& =\boldsymbol{\theta}_{\Sigma}^{\mathrm{MM}}+\int_{\partial \Sigma} \boldsymbol{d e} \boldsymbol{e}^{-1} \bullet F+\frac{2 \varepsilon}{\ell^{2}} \int_{\Sigma} \boldsymbol{d e} \boldsymbol{e}^{-1} \bullet T \wedge e^{T}, \\
& \left(\boldsymbol{\Theta}_{\Sigma}^{\mathrm{MM}}\right)^{\boldsymbol{u}}=\boldsymbol{\Theta}_{\Sigma}^{\mathrm{MM}}+\int_{\partial \Sigma} \boldsymbol{d} \theta\left(\boldsymbol{d u} \boldsymbol{u}^{-1} ; \bar{A}\right)-\int_{\Sigma} \boldsymbol{d} E\left(\boldsymbol{d u} \boldsymbol{u}^{-1} ; \bar{A}\right) . \\
& =\Theta_{\Sigma}^{\mathrm{MM}}+\int_{\partial \Sigma} \boldsymbol{d}\left(\boldsymbol{d e} \boldsymbol{e}^{-1} \bullet F\right)+\frac{2 \varepsilon}{\ell^{2}} \int_{\Sigma} \boldsymbol{d}\left(\boldsymbol{d e} \boldsymbol{e}^{-1} \bullet T \wedge e^{T}\right) .
\end{aligned}
$$

As per the DFM philosophy, section 4.2, these are none other than the field equations and presymplectic structure of the dressed Lagrangian

$$
\begin{aligned}
L_{\mathrm{MM}}^{\boldsymbol{u}}(\bar{A})=\bar{L}_{\mathrm{MM}}(\bar{\Gamma}) & =\sqrt{|\operatorname{det}(\boldsymbol{g})|} \frac{1}{2} \mathrm{~F} \bullet \mathrm{F}, \\
& =\sqrt{|\operatorname{det}(\boldsymbol{g})|} \frac{1}{2} \mathrm{R} \bullet \mathrm{R}-\frac{\varepsilon}{\ell^{2}}\left(\mathrm{R} \bullet d x \wedge d x^{T}-\frac{\varepsilon}{2 \ell^{2}} d x \wedge d x^{T} \bullet d x \wedge d x^{T}\right),
\end{aligned}
$$

where $\bar{L}_{\mathrm{MM}}$ is based on the polynomial (4.48). This is manifestly just the Lagrangian of GR in the 'metric' formulation. Which means in particular that $\left(\boldsymbol{\theta}_{\Sigma}^{\mathrm{MM}}\right) \boldsymbol{u}$ is simply the 
presymplectic potential of the metric formulation,

$$
\left(\boldsymbol{\theta}_{\Sigma}^{\mathrm{MM}}\right)^{\boldsymbol{u}}=\bar{\theta}_{\mathrm{MM}}(\boldsymbol{d} \bar{\Gamma} ; \bar{\Gamma})=\int_{\Sigma} \sqrt{|\operatorname{det}(\boldsymbol{g})|} \boldsymbol{d} \Gamma \bullet \mathrm{F}=\int_{\Sigma} \sqrt{|\operatorname{det}(\boldsymbol{g})|} \boldsymbol{d} \Gamma \bullet\left(\mathrm{R}-\frac{\varepsilon}{\ell^{2}} d x \wedge d x^{T}\right) .
$$

The associated 2-form is easily deduced. Equation (4.51) then gives the relation between the metric and tetrad potentials of pure GR, and its boundary term generalises in particular the (aptly named from the DFM viewpoint) "dressing 2-form" of DePaoli-Speziale [28] — see also [29, 30].

In the situation at hand, we have an occasion to see that the ambiguity in the choice of dressing field encodes a relevant gauge symmetry of the dressed theory: coordinate changes. Indeed, we identified the components of the soldering form $e=e^{a}{ }_{\mu}$ in a given coordinate system $\left\{x^{\mu}\right\}$ as a good SO-dressing field. In another coordinate system we have of course $e^{\prime}=e \xi$, i.e. $e^{\prime a}{ }_{\nu}=e^{a}{ }_{\mu} \xi^{\mu}{ }_{\nu}$, where $\xi=\xi^{\mu}{ }_{\nu} \in \mathrm{GL}(4)$ is the Jacobian of the coordinate change. The ambiguity in the choice of Lorentz dressing is thus parametrised by the group of local coordinate transformations $\mathcal{G}=\mathcal{G} \mathcal{L}:=\left\{\xi: U \rightarrow \mathrm{GL}(4) \mid \xi^{\gamma}=\xi\right\}$ acting as $\boldsymbol{u}^{\xi}=\boldsymbol{u} \xi$ and of course trivially on the Cartan connection $\bar{A}^{\xi}=\bar{A}$. The space of dressed Cartan connections $\overline{\mathcal{A}}^{u}=\bar{\Gamma}$ is then a $\mathcal{G}$-principal bundle, with a right action of $\mathcal{G}$ given by

$$
R_{\xi} \bar{A}^{u}=\left(\bar{A}^{u}\right)^{\xi}:=\xi^{-1} \bar{A}^{u} \xi+\xi^{-1} d \xi \Rightarrow\left\{\begin{array}{l}
\left(A^{u}\right)^{\xi}=\Gamma^{\xi}=\xi^{-1} \Gamma \xi+\xi^{-1} d \xi \\
\left(e^{u}\right)^{\xi}=(d x)^{\xi}=\xi^{-1} d x
\end{array}\right.
$$

By (4.48), the dressed Lagrangian (4.53) has trivial $\mathcal{G}$-equivariance $R_{\xi}^{\star} \bar{L}_{\mathrm{MM}}=\bar{L}_{\mathrm{MM}}$. So, for $\varkappa=\varkappa_{\nu}^{\mu} \in \operatorname{Lie} \mathcal{G}$ generating $\varkappa^{v} \in V\left(\overline{\mathcal{A}}^{u}\right)$ and by (4.19), the dressed Noether charge is:

$$
\begin{aligned}
\bar{Q}_{\Sigma}^{\mathrm{MM}}\left(\varkappa ; \bar{A}^{\boldsymbol{u}}\right) & =\int_{\partial \Sigma} \bar{\theta}_{\mathrm{MM}}\left(\varkappa ; \bar{A}^{\boldsymbol{u}}\right)-\int_{\Sigma} \bar{E}_{\mathrm{MM}}\left(\varkappa ; \bar{A}^{\boldsymbol{u}}\right), \\
& =\int_{\partial \Sigma} \sqrt{|\operatorname{det}(\boldsymbol{g})|} \varkappa \bullet \mathrm{F}+\frac{2 \varepsilon}{\ell^{2}} \int_{\Sigma} \sqrt{|\operatorname{det}(\boldsymbol{g})|} \varkappa \bullet \mathrm{T} \wedge d x^{T} .
\end{aligned}
$$

It satisfies $\iota_{\varkappa^{v}}\left(\boldsymbol{\Theta}_{\Sigma}^{\mathrm{MM}}\right)^{\boldsymbol{u}}=-\boldsymbol{d} \bar{Q}_{\Sigma}^{\mathrm{MM}}\left(\varkappa ; \bar{A}^{\boldsymbol{u}}\right)$ and via (4.20) the dressed presymplectic 2-form induces the Poisson bracket

$$
\left\{\bar{Q}_{\Sigma}^{\mathrm{MM}}\left(\varkappa ; \bar{A}^{u}\right), \bar{Q}_{\Sigma}^{\mathrm{MM}}\left(\varkappa^{\prime} ; \bar{A}^{u}\right)\right\}:=\left(\Theta_{\Sigma}^{\mathrm{MM}}\right) \boldsymbol{u}\left(\varkappa^{v}, \varkappa^{\prime v}\right)=\bar{Q}_{\Sigma}^{\mathrm{MM}}\left(\left[\varkappa, \varkappa^{\prime}\right] ; \bar{A}^{u}\right),
$$

so that the Poisson algebra of dressed (metric) charges is isomorphic to the Lie algebra of coordinate changes Lie $\mathcal{G}$. The same relations hold for field-dependent parameters $\varkappa \in$ Lie $\mathcal{G}$, where $\mathcal{G}=\mathcal{G} \mathcal{L}$ is the gauge group of the $\mathcal{G}$-bundle $\overline{\mathcal{A}}^{u}$, even though in this case the charge is non-integrable $\iota_{\varkappa^{v}}\left(\Theta_{\Sigma}^{\mathrm{MM}}\right)^{\boldsymbol{u}}=-\boldsymbol{d} \bar{Q}_{\Sigma}^{\mathrm{MM}}\left(\underline{\varkappa} ; \bar{A}^{\boldsymbol{u}}\right)$.

Charges (4.45) of $L_{\mathrm{MM}}$, like those (3.59) of $L_{\mathrm{GR}}$ in section 3.3.2, could be interpreted via the affine ansatz (3.26) $\bar{A}=\bar{A}_{0}+\bar{\alpha}$. But when attempting to do the same with the charges (4.56) above, echoing the caveats at the end of section 4.2 , we must exercise care as the ansatz affects the dressing field $\boldsymbol{u}(\bar{A})=\boldsymbol{e}$ as well. Splitting in particular the tetrad as background and fluctuation, $\boldsymbol{e}=\boldsymbol{e}_{0}+\epsilon$, induces a corresponding splitting of the metric $\boldsymbol{g}=\boldsymbol{g}_{0}+\boldsymbol{h}$ which must then be plugged in the on-shell expression for $\bar{Q}_{\Sigma}^{\mathrm{MM}}\left(\varkappa ; \bar{A}^{\boldsymbol{u}}\right)$. 
Although doable, it is cumbersome. One may rely on a simpler heuristics to interpret the dressed charge: on-shell (or simply in the normal case) these are

$$
\begin{aligned}
\bar{Q}_{\Sigma}^{\mathrm{MM}}\left(\varkappa ; \bar{A}^{\boldsymbol{u}}\right) & =\int_{\partial \Sigma} \sqrt{|\operatorname{det}(\boldsymbol{g})|} \varkappa \bullet \mathrm{F}_{\mid \mathcal{S}} \\
& =\int_{\partial \Sigma} \sqrt{|\operatorname{det}(\boldsymbol{g})|} \varkappa \bullet\left(\mathrm{R}-\frac{\varepsilon}{\ell^{2}} d x \wedge d x^{T}\right), \\
& =\int_{\partial \Sigma} \sqrt{|\operatorname{det}(\boldsymbol{g})|}\left(\varepsilon_{\mu \nu \sigma \rho} \alpha^{\mu \nu} \frac{1}{2} \mathrm{R}_{\alpha \beta}^{\sigma \rho}-\frac{\varepsilon}{\ell^{2}} \varkappa^{\mu \nu} \varepsilon_{\mu \nu \alpha \beta}\right) d x^{\alpha} \wedge d x^{\beta} .
\end{aligned}
$$

Now if one considers $\varkappa=\partial \zeta=\partial_{\nu} \zeta^{\mu}$ with $\zeta$ the components of a Killing vector field of $\boldsymbol{g}$, the above expression is a generalised Komar integral. It reproduces the result for 4D Zumino-Lovelock theory gravity (also known as Gauss-Bonnet gravity) obtained in [98] eq. (17)-(20), and generalises the usual Komar mass as given in [99] (definition 4.6, eq. (4.8) p. 460) - known to coincide with the Newtonian mass and ADM mass for (stationnary) asymptotically flat spacetimes $M$ and to vanish if and only if $M$ is flat (Lemma 4.10, Theorem 4.13 and Theorem 4.11 in [99]). The charge (4.58) gives a good notion of mass and angular momentum (according to the nature of $\zeta$ ) in gravity with $\Lambda \neq 0$ as it vanishes on the (A)dS groundstate of the theory, i.e. the homogeneous space of the underlying Cartan geometry.

The general formulae (4.17) applied here give the field-dependent coordinate transformations of the field equations and presymplectic structure of $\bar{L}_{\mathrm{MM}}$

$$
\begin{aligned}
& {\left[\boldsymbol{E}_{\mathrm{MM}}^{\boldsymbol{u}}\right]^{\xi}=\boldsymbol{E}_{\mathrm{MM}}^{\boldsymbol{u}}-d\left(\frac{2 \varepsilon}{\ell^{2}} \sqrt{|\operatorname{det}(\boldsymbol{g})|} \boldsymbol{d} \boldsymbol{\xi} \boldsymbol{\xi}^{-1} \bullet \mathrm{T} \wedge d x^{T}\right),} \\
& =\boldsymbol{E}_{\mathrm{YM}}^{u}+d\left(\frac{2 \varepsilon}{\ell^{2}} \stackrel{\circ}{\boldsymbol{\beta}} \bullet T \wedge e^{T}\right), \\
& {\left[\left(\boldsymbol{\theta}_{\Sigma}^{\mathrm{MM}}\right)^{\boldsymbol{u}}\right]^{\boldsymbol{\xi}}=\left(\boldsymbol{\theta}_{\Sigma}^{\mathrm{MM}}\right)^{\boldsymbol{u}}+\int_{\partial \Sigma} \sqrt{|\operatorname{det}(\boldsymbol{g})|} \boldsymbol{d} \boldsymbol{\xi} \boldsymbol{\xi}^{-1} \bullet \mathrm{F}} \\
& +\frac{2 \varepsilon}{\ell^{2}} \int_{\Sigma} \sqrt{|\operatorname{det}(\boldsymbol{g})|} \boldsymbol{d} \boldsymbol{\xi} \boldsymbol{\xi}^{-1} \bullet \mathrm{T} \wedge d x^{T}, \\
& =\left(\boldsymbol{\theta}_{\Sigma}^{\mathrm{MM}}\right)^{\boldsymbol{u}}-\int_{\partial \Sigma} \stackrel{\circ}{\boldsymbol{\beta}} \bullet F-\frac{2 \varepsilon}{\ell^{2}} \int_{\Sigma} \stackrel{\circ}{\boldsymbol{\beta}} \bullet T \wedge e^{T}, \\
& {\left[\left(\boldsymbol{\Theta}_{\Sigma}^{\mathrm{MM}}\right)^{\boldsymbol{u}}\right]^{\boldsymbol{\xi}}=\left(\boldsymbol{\Theta}_{\Sigma}^{\mathrm{MM}}\right)^{\boldsymbol{u}}+\int_{\partial \Sigma} \boldsymbol{d}\left\{\sqrt{|\operatorname{det}(\boldsymbol{g})|} \boldsymbol{d} \boldsymbol{\xi} \boldsymbol{\xi}^{-1} \bullet \mathrm{F}\right\}} \\
& +\frac{2 \varepsilon}{\ell^{2}} \int_{\Sigma} \boldsymbol{d}\left\{\sqrt{|\operatorname{det}(\boldsymbol{g})|} \boldsymbol{d} \boldsymbol{\xi} \boldsymbol{\xi}^{-1} \bullet \mathrm{T} \wedge d x^{T}\right\}, \\
& =\left(\boldsymbol{\Theta}_{\Sigma}^{\mathrm{MM}}\right)^{\boldsymbol{u}}-\int_{\partial \Sigma} \boldsymbol{d}(\stackrel{\circ}{\boldsymbol{\beta}} \bullet F)-\frac{2 \varepsilon}{\ell^{2}} \int_{\Sigma} \boldsymbol{d}\left(\stackrel{\circ}{\boldsymbol{\beta}} \bullet T \wedge e^{T}\right) .
\end{aligned}
$$

We provide the second line, where $\stackrel{\circ}{\boldsymbol{\beta}}=-\boldsymbol{e d} \boldsymbol{\xi} \boldsymbol{\xi}^{-\mathbf{1}} \boldsymbol{e}^{-1}$, for comparison with (4.42). The similarity is of course no accident as using the dressing $\boldsymbol{u}(\bar{A})=\boldsymbol{e}$ is equivalent to using the flat connection $\stackrel{\circ}{\omega}:=-\boldsymbol{d u} \boldsymbol{u}^{-1}$ on the $\mathcal{H}$-bundle $\overline{\mathcal{A}}$. One checks easily the two defining properties (2.17)-(2.18) of an Ehresmann connection:

$$
\begin{aligned}
& R_{\gamma}^{\star} \stackrel{\circ}{\omega}=-\boldsymbol{d} R_{\gamma}^{\star} e\left(R_{\gamma}^{\star} e\right)^{-1}=-\gamma^{-1} \boldsymbol{d e} e^{-1} \gamma=\gamma^{-1} \stackrel{\circ}{\gamma} \gamma, \quad \text { for } \gamma \in \mathcal{H}=\mathcal{S O}(1,3), \\
& \stackrel{\circ}{\boldsymbol{\omega}}\left(\chi^{v}\right)=-\boldsymbol{d e e}^{-1}\left(\chi^{v}\right)=-\chi^{v}(\boldsymbol{e}) \boldsymbol{e}^{-1}=-(-\chi \boldsymbol{e}) \boldsymbol{e}^{-1}=\chi \in \operatorname{LieSO}(1,3) .
\end{aligned}
$$


And the flatness condition is trivial $\boldsymbol{d} \stackrel{\circ}{\boldsymbol{\omega}}+\frac{1}{2}[\stackrel{\circ}{\boldsymbol{\omega}}, \stackrel{\circ}{\boldsymbol{\omega}}]=\boldsymbol{d e d e} \boldsymbol{e}^{-1}+\boldsymbol{d e} \boldsymbol{e}^{-1} \boldsymbol{d e} \boldsymbol{e}^{-1} \equiv 0$. A fielddependent coordinate change $\boldsymbol{u}^{\prime}=\boldsymbol{u} \boldsymbol{\xi}$ induces an affine shift of flat connection $\stackrel{\circ}{\boldsymbol{\omega}}^{\prime}=\dot{\boldsymbol{\omega}}+\stackrel{\circ}{\boldsymbol{\beta}}$.

In good illustration of the general comment 1 of section 4.2 , we have no trouble appreciating that $\mathcal{G}=\mathcal{G} \mathcal{L}$ is not a physical transformation group, and doesn't permute points in the physical phase space $\mathcal{S} / \mathcal{S O} \simeq \mathcal{S}^{u} / \mathcal{G}$. The question of the physical relevance (or signature) of $\mathcal{G}$ is of the same nature as that of the original $\mathcal{S O}$ gauge symmetry, as illustrated above in the discussion of the interpretation of dressed charges. Finally, (4.59) shows that as a boundary problem for $\mathcal{S O}$ is solved by dressing, another emerges for $\mathcal{G}=\mathcal{G} \mathcal{L}$ which is very likely a substantial symmetry of the dressed/metric theory, suggesting that the boundary problem signals the non-locality, or non-separability, of gravitational physics.

Theory coupled to spinors: according to the DFM philosophy, the presence of the tetrad as a local dressing field that can be used to rewrite the theory in terms of Lorentz-invariant variables makes $\mathcal{S O}(1,3)$ an artificial symmetry of both pure GR and of GR coupled with bosonic and scalar fields (EM field, fluids, dust...). This is an interesting contrast with, say, the EM case: in the pure gauge theory no local dressing built from the connection exists so $\mathcal{U}(1)$ is substantial, while in $\mathbb{C}$-scalar EM a local dressing is extracted from the matter sector so that $\mathcal{U}(1)$ is artificial.

Yet, in the same way that the coupling of the EM field to spinors makes $\mathcal{U}(1)$ substantial again, the coupling of gravity to spinors a priori changes the verdict on $\mathcal{S O}(1,3)$. The tetrad being $G L$-valued, and for lack of finite dimensional spin representations of $G L$, it cannot be used to produce a Lorentz-invariant spinor: we cannot write $\psi^{\boldsymbol{u}}=\rho(\boldsymbol{u})^{-1} \psi=\rho(\boldsymbol{e})^{-1} \psi$. This lack of local dressing for the whole theory strongly suggest that the Lorentz gauge symmetry $\mathcal{S O}(1,3)$ is substantial in GR + spinors, and the associated boundary problem a reflection of the non-locality/non-separability of gravitational physics.

We may nuance this conclusion in light of the fact that, so long as one works on $U \subset \mathcal{M}$, one can decompose the tetrad as $\boldsymbol{e}=\boldsymbol{u} \boldsymbol{t}$, where $\boldsymbol{u} \in H=\mathrm{SO}(1,3)$ and $\boldsymbol{t}=\boldsymbol{t}^{b}{ }_{\mu}$ has the same d.o.f as the metric field and is s.t. $\boldsymbol{g}=\boldsymbol{t}^{T} \eta \boldsymbol{t}$. For details and references on such a decomposition, which relies on the Schweinler-Wigner orthogonalization procedure, we refer to section 4.3 of [52] — see also the end of section 2 and footnote 12 of [55]. It is akin to the polar decomposition of the $\mathbb{C}$-scalar field in EM: $\boldsymbol{u}$ carries the gauge representation, $\boldsymbol{u}^{\gamma}=\gamma^{-1} \boldsymbol{u}$ with $\gamma \in \mathcal{S O}(1,3)$, so that $\boldsymbol{u}=\boldsymbol{u}(e): \Phi \rightarrow \mathcal{D} r[H, H]$ is a (minimal) local Lorentz dressing field.

As per section 4.2, it can be used to obtain the dressed Lagrangian,

$$
\begin{aligned}
& L_{\mathrm{GR}}^{\boldsymbol{u}}=\mathrm{F}_{\boldsymbol{u}}^{\star} L_{\mathrm{GR}} \quad \text { i.e. } \quad L_{\mathrm{GR}}^{\boldsymbol{u}}(\phi)=L_{\mathrm{MM}}\left(\bar{A}^{\boldsymbol{u}}\right)+L_{\mathrm{Dirac}}\left(\bar{A}^{\boldsymbol{u}}, \psi^{\boldsymbol{u}}\right) \\
& =\frac{1}{2} F^{u} \bullet F^{u}+\left\langle\psi^{u}, \not D^{u} \psi^{u}\right\rangle \quad \in \Omega_{\text {basic }}^{0}(\Phi) .
\end{aligned}
$$

where invariant spinor fields $\psi^{\boldsymbol{u}}:=\rho(\boldsymbol{u})^{-1} \psi$ couples to gravity via the invariant gauge field $A^{u}:=\boldsymbol{u}^{-1} A \boldsymbol{u}+\boldsymbol{u}^{-1} d \boldsymbol{u}$ (the Lorentz part of the dressed Cartan connection $\bar{A}^{u}$ whose soldering part is $\left.e^{\boldsymbol{u}}=t=\boldsymbol{t} \cdot d x\right)$. From the variation of (4.60), $\boldsymbol{d} L_{\mathrm{GR}}^{\boldsymbol{u}}=\boldsymbol{E}_{\mathrm{GR}}^{\boldsymbol{u}}+d \boldsymbol{\theta}_{\mathrm{GR}}^{\boldsymbol{u}}=$ $E_{\mathrm{GR}}\left(\boldsymbol{d} \phi^{u} ; \phi^{\boldsymbol{u}}\right)+d \theta_{\mathrm{GR}}\left(\boldsymbol{d} \phi^{u} ; \phi^{\boldsymbol{u}}\right)$, one gets the associated field equations and basic presymplectic structure, which by (4.11)-(4.12) and given (3.54)-(3.55) — or by (3.62)-(3.64) 
together with the rule of thumb $\gamma \rightarrow \boldsymbol{u}$ - are:

$$
\begin{aligned}
\boldsymbol{E}_{\mathrm{GR}}^{\boldsymbol{u}}= & \boldsymbol{E}_{\mathrm{GR}}+d E_{\mathrm{GR}}\left(\boldsymbol{d} \boldsymbol{u} \boldsymbol{u}^{-1} ; \phi\right), \quad \in \Omega_{\mathrm{basic}}^{1}(\Phi), \\
= & \boldsymbol{E}_{\mathrm{GR}}-d\left\{\frac{2 \varepsilon}{\ell^{2}}\left(\boldsymbol{d} \boldsymbol{u} \boldsymbol{u}^{-1} \bullet T \wedge e^{T}\right)+\operatorname{Tr}\left(\left|\rho_{*}\left(\boldsymbol{d} \boldsymbol{u} \boldsymbol{u}^{-1}\right) \psi\right\rangle\langle * \gamma \psi|\right)\right\} \\
\left(\boldsymbol{\theta}_{\Sigma}^{\mathrm{GR}}\right)^{\boldsymbol{u}}= & \boldsymbol{\theta}_{\Sigma}^{\mathrm{GR}}+\int_{\partial \Sigma} \theta_{\mathrm{GR}}\left(\boldsymbol{d} \boldsymbol{u} \boldsymbol{u}^{-1} ; \phi\right)-\int_{\Sigma} E_{\mathrm{GR}}\left(\boldsymbol{d} \boldsymbol{u} \boldsymbol{u}^{-1} ; \phi\right), \quad \in \Omega_{\mathrm{basic}}^{1}(\Phi), \\
= & \boldsymbol{\theta}_{\Sigma}^{\mathrm{GR}}+\int_{\partial \Sigma} \boldsymbol{d} \boldsymbol{u} \boldsymbol{u}^{-1} \bullet F \\
& +\int_{\Sigma} \frac{2 \varepsilon}{\ell^{2}} \boldsymbol{d} \boldsymbol{u} \boldsymbol{u}^{-1} \bullet T \wedge e^{T}-\operatorname{Tr}\left(\left|\rho_{*}\left(\boldsymbol{d} \boldsymbol{u} \boldsymbol{u}^{-1}\right) \psi\right\rangle\langle * \gamma \psi|\right), \\
\left(\boldsymbol{\Theta}_{\Sigma}^{\mathrm{GR}}\right)^{\boldsymbol{u}}= & \boldsymbol{\Theta}_{\Sigma}^{\mathrm{GR}}+\int_{\partial \Sigma} \boldsymbol{d} \theta_{\mathrm{GR}}\left(\boldsymbol{d} \boldsymbol{u} \boldsymbol{u}^{-1} ; \phi\right)-\int_{\partial \Sigma} \boldsymbol{d} E_{\mathrm{GR}}\left(\boldsymbol{d} \boldsymbol{u} \boldsymbol{u}^{-1} ; \phi\right), \quad \in \Omega_{\mathrm{basic}}^{2}(\Phi), \\
= & \boldsymbol{\Theta}_{\Sigma}^{\mathrm{GR}}+\int_{\partial \Sigma} \boldsymbol{d}\left(\boldsymbol{d} \boldsymbol{u} \boldsymbol{u}^{-1} \bullet F\right) \\
& +\int_{\Sigma} \frac{2 \varepsilon}{\ell^{2}} \boldsymbol{d}\left(\boldsymbol{d} \boldsymbol{u} \boldsymbol{u}^{-1} \bullet T \wedge e^{T}\right)-\boldsymbol{d} \operatorname{Tr}\left(\left|\rho_{*}\left(\boldsymbol{d} \boldsymbol{u} \boldsymbol{u}^{-1}\right) \psi\right\rangle\langle * \gamma \psi|\right)
\end{aligned}
$$

Unfortunately, (4.62)-(4.63) do not really solve the boundary problem, as these are at best valid only locally, i.e. on a single coordinate patch. Indeed, $\boldsymbol{u}$ and by extension the composite fields and other quantities built from it, depend on the coordinate chart $\left\{x^{\mu}\right\}$ on $U \subset M$ in such a way that they have no determined well-behaved transformation law under coordinate changes (as explained in [52]). Which makes them ill-defined as global geometrical objects on $M$. This also means that our general discussion about the ambiguity in the choice of dressing doesn't apply in this case, and in particular no charges associated with $\mathcal{G} \mathcal{L}(4)$ can be assigned via $\left(\boldsymbol{\theta}_{\Sigma}^{\mathrm{GR}}\right)^{\boldsymbol{u}}$.

This construction of $\boldsymbol{u}$ from a decomposition of the tetrad seems to bear some relation to the attempts - pioneered in the ' 50 s and ' 60 s by DeWitt, Ogievetsky and Polubarinov — to build spinors without introducing Lorentz gauge symmetry. See [100] for a review with an extensive bibliography. Upon mild restrictions on the admissible coordinate systems, the coordinate transformation law for such spinors is formally attainable, but only in the weak field regime around a flat background, i.e when $\boldsymbol{g}$ is a small perturbation around $\eta$. Even then, the transformation law is metric dependent and highly non-linear. It is thus not obvious that such a framework could be satisfactory in the strong field regime of GR, or that it can be accommodated to QFT in curved spacetime. A prudent commitment to the initial assessment that $\mathcal{S O}(1,3)$ is substantial in spinorial gravity therefore seems reasonable.

The absence of dressing for the theory 'gravity + spinors' is less of a problem if one is mainly interested in charges and their Poisson algebra: on-shell, charges in the coupled theory (3.56) are the same as charges of pure gravity (4.45) and reduce to boundary terms, $Q_{\Sigma}^{\mathrm{GR}}(\chi ; \phi)=\int_{\partial \Sigma} \chi \bullet F_{\mid \mathcal{S}}$. Which makes sense as the matter sources presumably have compact support while their gravitational field propagates and reaches infinity. But then the on-shell charges of the coupled theory can be dressed as in (4.56) giving $Q_{\Sigma}^{\mathrm{GR}}(\varkappa ; \phi)=$ $\int_{\partial \Sigma} \sqrt{|\operatorname{det}(\boldsymbol{g})|} \varkappa \bullet \mathrm{F}_{\mid \mathcal{S}}$, and interpreted as in the free case, while their Poisson bracket is on-shell (4.57). 


\section{Conclusion}

In this note we made decisive use of the bundle geometry of field space which, articulated with covariant phase space methods, allows to give a series of general results on the presymplectic structure of invariant, matter coupled, gauge theories. That is, given the Lagrangian $L$ of any such theory, we gave the off-shell expression of the Noether charges for field-independent (3.6) and field-dependent (3.19) parameters, as well as their Poisson bracket (3.9)-(3.25) induced by the presymplectic 2 -form. We also gave the general fielddependent gauge transformations of the presymplectic potential (3.13) and 2-form (3.17), which exhibit the boundary problem in full generality. We stress that the only computation needed to apply these results in any given example, is to derive the field equations $\boldsymbol{E}$ and the presymplectic potential $\boldsymbol{\theta}$ from $L$. Which we did in the case of YM theory (section 3.3.1) and GR in its Cartan geometric formulation (section 3.3.2), thereby recovering standard results. In passing, we noticed that using the affine structure of the space of connections $\mathcal{A}$, it is possible to split the Noether charge as a background contribution and a measurable contribution associated with a Killing symmetry of the background gauge field. This generalises the approach of Abbott \& Deser [34, 35].

Emphasis on the bundle geometry of field space $\Phi$ allows to appreciate that solving the boundary problem boils down to one thing only: finding ways to build the basic counterpart of a given variational form on $\Phi$. We reminded that variational connections and the DFM (a.k.a edge modes) are means to do just that, and conducted a systematic comparative analysis of both in sections 2.2 and 2.3. We could apply this to produce the general basic presymplectic structure of an invariant theory as obtained via connections (4.7) and via dressings (4.12). Special applications to YM theory and GR reproduce several results of the literature, e.g. $[5,8,9]$, and in particular the DFM gives from first principle the unambiguous relations (4.51)-(4.52) between the presymplectic structures of GR in the tetrad and metric formulations, generalising the dressing 2-form of [28] — see also [29, 30].

The most relevant conceptual difference between the two approaches - beside the fact that the existence of dressing fields imposes stronger topological constraints on field space than (non-flat) connections - resides in how their respective ambiguities arise. Any two choices of connections are related via an affine shift by a Ad-tensorial 1-form on $\Phi$, leading to relations like $(2.30) /(4.8)$, which is not associated with any relevant new symmetries. On the contrary, the ambiguity in the choice of dressings, while indeed manifesting itself as a special case of affine shift of the associated connections (2.59), is more generally encoded by a group $\mathcal{G}$ that may be a relevant symmetry of the dressed theory $L^{u}$. This, the DFM shows via (2.43), is a gauge symmetry that doesn't enjoy a more direct physical interpretation than the original gauge symmetry $\mathcal{H}$ of $L$, but whose relevance may show through the associated dressed charges (4.19). As a matter of fact, in GR these dressed charges give (generalised) Komar integrals (4.58). This, we argue, also clarifies the status of the "surface symmetries" of the edge mode literature, which are exactly instances of $\mathcal{G}$-symmetries. The corollary is of course that $\mathcal{G}$ may manifest its own boundary problem, unless the construction of a dressing field in the theory under consideration is free enough of any ambiguities - as it arguably is the case for example in $\mathbb{C}$-scalar electrodynamics, as discussed at the end of section 4.3.1. 
We remark that following the template of sections 2 and 3 above, one may analyse the presymplectic structure of twisted gauge theories. These are gauge theories whose configuration space is $\tilde{\Phi}:=\tilde{\mathcal{A}} \times \Gamma(\tilde{E})$ with $\tilde{\mathcal{A}}$ the space of twisted connections on a standard $H$-principal bundle $\mathcal{P}$ - generalising Ehresmann and Cartan connections - and $\Gamma(\tilde{E})$ the space of sections of twisted bundles associated with $\mathcal{P}$ built via 1-cocycles of the structure group $H$ - extensions of standard associated bundles built via representations. We briefly evoked these notions in sections 2.1 and 2.2.2 in the context of the bundle $\Phi$, but these have finite dimensional precursors whose elementary theory is described in [47]. As shown there, conformal gravity is an unexpected example of twisted gauge theory, the (dressed) conformal Cartan connection - otherwise known as the tractor or twistor connection being a twisted connection transforming via a cocycle of the group of Weyl rescalings (a subgroup of the structure group of the conformal Cartan bundle [67]). The presymplectic structure of conformal gravity - possibly coupled to twistors (or conformal tractors [101]) - would then follow as an immediate application of the suggested extension of the present work to twisted gauge theories

A more immediate direction we wish to explore is to consider the case of non-invariant theories, i.e. to include classical gauge anomalies. In [33], this was done for pure gauge theories, and only charges for field-independent gauge parameters were considered. We endeavor to maximally generalise the latter work to encompass non-invariant coupled theories, to include the case where the symmetry under consideration is Diff $(M)$ rather than an internal gauge group, and to extend the discussion to charges for field-dependent gauge parameters and their Poisson bracket. The latter should be a generalisation of the BarnichTroessaert bracket proposed in [102]. This, by the way, should provide a cross-check of the recent [65] whose authors suggest they have found such a generalised bracket that is not centrally extended, while generically we expect ours to be. As a matter of direct applications, we expect our Poisson algebras of charges in $3 \mathrm{D}$ and $4 \mathrm{D}$ gravity with $\Lambda \neq 0$ to extend the $\mathfrak{b} \mathfrak{m} \mathfrak{s} 3$ and $\mathfrak{b m s} 4$ algebras, likely making contact with the $\Lambda$-bms 4 algebra of [103]. Working out the Poisson algebra of charge of conformal gravity, in the framework alluded to above, would certainly reproduce or a least make contact with the so-called Weyl-bms algebra of [104].

\section{Acknowledgments}

J.F. and N.B. are supported by the Fonds de la Recherche Scientifique - FNRS under grant PDR No. T.0022.19 ("Fundamental issues in extended gravitational theories"), and J.F. is also supported by the FNRS grant MIS No. F.4503.20 ("HighSpinSymm").

\section{A Proof of formula (2.27)}

Given $\boldsymbol{\alpha} \in \Omega_{\text {inv }}^{1}(\Phi)$, the infinitesimal version of its trivial $\mathcal{H}$-equivariance reads $\boldsymbol{L}_{\chi^{v}} \boldsymbol{\alpha}=$ $\iota_{\chi^{v}} \boldsymbol{d} \boldsymbol{\alpha}+\boldsymbol{d} \iota_{\chi^{v}} \boldsymbol{\alpha} \equiv 0$ for any $\chi \in$ Lie $\mathcal{H}$. So, using $\left[\boldsymbol{L}_{\boldsymbol{X}}, \iota_{\boldsymbol{Y}}\right]=\iota_{[\boldsymbol{X}, \boldsymbol{Y}]}$, we have the identity

$$
\boldsymbol{d} \boldsymbol{\alpha}\left(\chi^{v}, \eta^{v}\right)=\iota_{\eta^{v}} \iota_{\chi^{v}} \boldsymbol{d} \boldsymbol{\alpha}=-\eta^{v} \boldsymbol{d} \iota_{\chi^{v}} \boldsymbol{\alpha}=-\boldsymbol{L}_{\eta^{v}} \iota_{\chi^{v}} \boldsymbol{\alpha}=-\iota_{\chi^{v}} \boldsymbol{L}_{\eta^{v}} \boldsymbol{\alpha}-\iota_{\left[\eta^{v}, \chi^{v}\right]} \boldsymbol{\alpha}=\iota_{[\chi, \eta]^{v}} \boldsymbol{\alpha} .
$$


Its covariant derivative is $\boldsymbol{D}^{\boldsymbol{\omega}} \boldsymbol{\alpha}:=\left.(\boldsymbol{d} \boldsymbol{\alpha}) \circ\right|^{h} \in \Omega_{\text {basic }}^{2}(\Phi)$. Its horizontal counterpart is $\boldsymbol{\alpha}^{h}:=\left.\boldsymbol{\alpha} \circ\right|^{h} \in \Omega_{\text {basic }}^{1}(\Phi)$. And since on basic forms the covariant derivative reduces to the exterior derivative, $\boldsymbol{D}^{\boldsymbol{\omega}} \boldsymbol{\alpha}^{h}=\boldsymbol{d} \boldsymbol{\alpha}^{h} \in \Omega_{\text {basic }}^{2}(\Phi)$. We want to prove that

$$
\boldsymbol{D}^{\boldsymbol{\omega}} \boldsymbol{\alpha}=\boldsymbol{d} \boldsymbol{\alpha}^{h}+\iota_{[\Omega]^{v}} \boldsymbol{\alpha} .
$$

Now, we have on the one hand, by the Koszul formula and (2.19):

$$
\begin{aligned}
& \boldsymbol{d} \boldsymbol{\alpha}_{\mid \phi}^{h}\left(\boldsymbol{X}_{\phi}, \boldsymbol{Y}_{\phi}\right)=\boldsymbol{X} \cdot \boldsymbol{\alpha}_{\mid \phi}^{h}\left(\boldsymbol{Y}_{\phi}\right)-\boldsymbol{Y} \cdot \boldsymbol{\alpha}_{\mid \phi}^{h}\left(\boldsymbol{X}_{\phi}\right)-\boldsymbol{\alpha}_{\mid \phi}^{h}\left([\boldsymbol{X}, \boldsymbol{Y}]_{\phi}\right), \\
& =\underline{X} \cdot \boldsymbol{\alpha}_{\mid \phi}\left(\underline{\boldsymbol{Y}_{\phi}}-\left[\boldsymbol{\omega}_{\mid \phi}\left(\boldsymbol{Y}_{\phi}\right)\right]_{\phi}^{v}\right)-\underline{\boldsymbol{Y} \cdot \boldsymbol{\alpha}_{\mid \phi}}\left(\underline{\boldsymbol{X}_{\phi}}-\left[\boldsymbol{\omega}_{\mid \phi}\left(\boldsymbol{X}_{\phi}\right)\right]_{\phi}^{v}\right) \\
& -\boldsymbol{\alpha}_{\mid \phi}\left(\underline{[X, Y]_{\phi}}-\left[\boldsymbol{\omega}_{\mid \phi}\left(\left[\boldsymbol{X}, \boldsymbol{Y}_{\phi}\right)\right]_{\phi}^{v}\right)\right. \text {, } \\
& =\underline{\boldsymbol{d} \boldsymbol{\alpha}_{\mid \phi}\left(\boldsymbol{X}_{\phi}, \boldsymbol{Y}_{\phi}\right)}-\boldsymbol{X} \cdot \boldsymbol{\alpha}_{\mid \underline{\phi}}\left(\left[\boldsymbol{\omega}_{\mid \underline{\phi}}\left(\boldsymbol{Y}_{\underline{\phi}}\right)\right]_{\underline{\phi}}^{v}\right)+\boldsymbol{Y} \cdot \boldsymbol{\alpha}_{\mid \underline{\phi}}\left(\left[\boldsymbol{\omega}_{\mid \underline{\phi}}\left(\boldsymbol{X}_{\underline{\phi}}\right)\right]_{\underline{\phi}}^{v}\right) \\
& +\boldsymbol{\alpha}_{\mid \phi}\left(\left[\boldsymbol{\omega}_{\mid \phi}\left([\boldsymbol{X}, \boldsymbol{Y}]_{\phi}\right)\right]_{\phi}^{v}\right) \text {. }
\end{aligned}
$$

The underlined $\phi$ in the two central terms means that the variational vector fields acting as a differential operator 'see' all the $\phi$ 's. On the other hand, we have

$$
\begin{aligned}
\boldsymbol{D}^{\omega} \boldsymbol{\alpha}_{\mid \phi}\left(\boldsymbol{X}_{\phi}, \boldsymbol{Y}_{\phi}\right):= & \boldsymbol{d} \boldsymbol{\alpha}_{\mid \phi}\left(\boldsymbol{X}_{\phi}^{h}, \boldsymbol{Y}_{\phi}^{h}\right)=\boldsymbol{d} \boldsymbol{\alpha}_{\mid \phi}\left(\boldsymbol{X}_{\phi}-\left[\boldsymbol{\omega}_{\mid \phi}\left(\boldsymbol{X}_{\phi}\right)\right]_{\phi}^{v}, \boldsymbol{Y}_{\phi}-\left[\boldsymbol{\omega}_{\mid \phi}\left(\boldsymbol{Y}_{\phi}\right)\right]_{\phi}^{v}\right) \\
= & \boldsymbol{d} \boldsymbol{\alpha}_{\mid \phi}\left(\boldsymbol{X}_{\phi}, \boldsymbol{Y}_{\phi}\right)-\boldsymbol{d} \boldsymbol{\alpha}_{\mid \phi}\left(\left[\boldsymbol{\omega}_{\mid \phi}\left(\boldsymbol{X}_{\phi}\right)\right]_{\phi}^{v}, \boldsymbol{Y}_{\phi}\right) \\
& -\boldsymbol{d} \boldsymbol{\alpha}_{\mid \phi}\left(\boldsymbol{X}_{\phi},\left[\boldsymbol{\omega}_{\mid \phi}\left(\boldsymbol{Y}_{\phi}\right)\right]_{\phi}^{v}\right)+\boldsymbol{d} \boldsymbol{\alpha}_{\mid \phi}\left(\left[\boldsymbol{\omega}_{\mid \phi}\left(\boldsymbol{X}_{\phi}\right)\right]_{\phi}^{v},\left[\boldsymbol{\omega}_{\mid \phi}\left(\boldsymbol{Y}_{\phi}\right)\right]_{\phi}^{v}\right) \\
= & \boldsymbol{d} \boldsymbol{\alpha}_{\mid \phi}\left(\boldsymbol{X}_{\phi}, \boldsymbol{Y}_{\phi}\right)+\iota_{\boldsymbol{Y}} \boldsymbol{d}\left(\boldsymbol{\alpha}_{\mid \phi}\left(\left[\omega_{\mid \phi}\left(\boldsymbol{X}_{\phi}\right)\right]_{\phi}^{v}\right)\right) \\
& -\iota_{\boldsymbol{X}} \boldsymbol{d}\left(\boldsymbol{\alpha}_{\mid \phi}\left(\left[\omega_{\mid \phi}\left(\boldsymbol{Y}_{\phi}\right)\right]_{\phi}^{v}\right)\right)+\boldsymbol{d} \boldsymbol{\alpha}_{\mid \phi}\left(\left[\boldsymbol{\omega}_{\mid \phi}\left(\boldsymbol{X}_{\phi}\right)\right]_{\phi}^{v},\left[\boldsymbol{\omega}_{\mid \phi}\left(\boldsymbol{Y}_{\phi}\right)\right]_{\phi}^{v}\right) \\
= & \boldsymbol{d} \boldsymbol{\alpha}_{\mid \phi}\left(\boldsymbol{X}_{\phi}, \boldsymbol{Y}_{\phi}\right)+\boldsymbol{Y} \cdot\left(\boldsymbol{\alpha}_{\mid \phi}\left(\left[\omega_{\mid \phi}\left(\boldsymbol{X}_{\phi}\right)\right]_{\phi}^{v}\right)\right) \\
& -\boldsymbol{X} \cdot\left(\boldsymbol{\alpha}_{\mid \phi}\left(\left[\omega_{\mid \phi}\left(\boldsymbol{Y}_{\phi}\right)\right]_{\phi}^{v}\right)\right)+\boldsymbol{\alpha}_{\mid \phi}\left(\left[\boldsymbol{\omega}_{\mid \phi}\left(\boldsymbol{X}_{\phi}\right), \boldsymbol{\omega}_{\mid \phi}\left(\boldsymbol{Y}_{\phi}\right)\right]_{\phi}^{v}\right) .
\end{aligned}
$$

In the equality before last above, we have used $\boldsymbol{L}_{\chi^{v}} \boldsymbol{\alpha}=0$ to rewrite the two central terms — and the last by (A.1) — thus considering $\left[\omega_{\mid \phi}\left(\boldsymbol{Y}_{\phi}\right)\right]$ as $\phi$-independent. Hence the fact that in the last equality, the variational vector fields only see the underlined $\phi$ 's. To further rewrite the last term we use,

$$
\begin{aligned}
\boldsymbol{\Omega}_{\mid \phi}\left(\boldsymbol{X}_{\phi}, \boldsymbol{Y}_{\phi}\right) & =\boldsymbol{d} \boldsymbol{\omega}_{\mid \phi}\left(\boldsymbol{X}_{\phi}, \boldsymbol{Y}_{\phi}\right)+\frac{1}{2}\left[\boldsymbol{\omega}_{\mid \phi}, \boldsymbol{\omega}_{\mid \phi}\right]\left(\boldsymbol{X}_{\phi}, \boldsymbol{Y}_{\phi}\right), \\
& =\boldsymbol{X} \cdot \boldsymbol{\omega}_{\mid \phi}\left(\boldsymbol{Y}_{\phi}\right)-\boldsymbol{Y} \cdot \boldsymbol{\omega}_{\mid \phi}\left(\boldsymbol{X}_{\phi}\right)-\boldsymbol{\omega}_{\mid \phi}\left([\boldsymbol{X}, \boldsymbol{Y}]_{\phi}\right)+\left[\boldsymbol{\omega}_{\mid \phi}\left(\boldsymbol{X}_{\phi}\right), \boldsymbol{\omega}_{\mid \phi}\left(\boldsymbol{Y}_{\phi}\right)\right]
\end{aligned}
$$

Then we get,

$$
\begin{aligned}
\boldsymbol{D}^{\omega} \boldsymbol{\alpha}_{\mid \phi}\left(\boldsymbol{X}_{\phi}, \boldsymbol{Y}_{\phi}\right)= & \boldsymbol{d} \boldsymbol{\alpha}_{\mid \phi}\left(\boldsymbol{X}_{\phi}, \boldsymbol{Y}_{\phi}\right)+\boldsymbol{Y} \cdot\left(\boldsymbol{\alpha}_{\mid \phi}\left(\left[\omega_{\mid \phi}\left(\boldsymbol{X}_{\phi}\right)\right]_{\phi}^{v}\right)\right)-\boldsymbol{X} \cdot\left(\boldsymbol{\alpha}_{\mid \phi}\left(\left[\omega_{\mid \phi}\left(\boldsymbol{Y}_{\phi}\right)\right]_{\phi}^{v}\right)\right) \\
& +\boldsymbol{\alpha}_{\mid \phi}\left(\left[\boldsymbol{Y} \cdot \boldsymbol{\omega}_{\mid \phi}\left(\boldsymbol{X}_{\phi}\right]_{\phi}^{v}\right)\right)-\boldsymbol{\alpha}_{\mid \phi}\left(\left[\boldsymbol{X} \cdot \boldsymbol{\omega}_{\mid \underline{\phi}}\left(\boldsymbol{Y}_{\phi}\right]_{\phi}^{v}\right)\right) \\
& +\boldsymbol{\alpha}_{\mid \phi}\left(\left[\boldsymbol{\omega}_{\mid \phi}\left([\boldsymbol{X}, \boldsymbol{Y}]_{\phi}\right]_{\phi}^{v}\right)\right)+\boldsymbol{\alpha}_{\mid \phi}\left(\left[\boldsymbol{\Omega}_{\mid \phi}\left(\boldsymbol{X}_{\phi}, \boldsymbol{Y}_{\phi}\right)\right]_{\phi}^{v}\right)
\end{aligned}
$$




$$
\begin{aligned}
= & \boldsymbol{d} \boldsymbol{\alpha}_{\mid \phi}\left(\boldsymbol{X}_{\phi}, \boldsymbol{Y}_{\phi}\right)+\boldsymbol{Y} \cdot \boldsymbol{\alpha}_{\mid \underline{\phi}}\left(\left[\boldsymbol{\omega}_{\mid \underline{\phi}}\left(\boldsymbol{X}_{\underline{\phi}}\right)\right]_{\phi}^{v}\right)-\boldsymbol{X} \cdot \boldsymbol{\alpha}_{\mid \phi}\left(\left[\boldsymbol{\omega}_{\mid \underline{\phi}}\left(\boldsymbol{Y}_{\underline{\phi}}\right)\right]_{\underline{\phi}}^{v}\right) \\
& +\boldsymbol{\alpha}_{\mid \phi}\left(\left[\boldsymbol{\omega}_{\mid \phi}\left([\boldsymbol{X}, \boldsymbol{Y}]_{\phi}\right)\right]_{\phi}^{v}\right)+\boldsymbol{\alpha}_{\mid \phi}\left(\left[\boldsymbol{\Omega}_{\mid \phi}\left(\boldsymbol{X}_{\phi}, \boldsymbol{Y}_{\phi}\right)\right]_{\phi}^{v}\right), \\
= & \boldsymbol{d} \boldsymbol{\alpha}_{\mid \phi}^{h}\left(\boldsymbol{X}_{\phi}, \boldsymbol{Y}_{\phi}\right)+\boldsymbol{\alpha}_{\mid \phi}\left(\left[\boldsymbol{\Omega}_{\mid \phi}\left(\boldsymbol{X}_{\phi}, \boldsymbol{Y}_{\phi}\right)\right]_{\phi}^{v}\right) .
\end{aligned}
$$

By (A.3) in the last equality, which proves (A.2).

\section{B The Abbott-Deser derivation of charges in YM theory}

One starts with the Yang-Mills equation for a gauge potential $A$ sourced by an external current $(n-1)$-form.

$$
D * F=J .
$$

Then one introduces the first ansatz, i.e. that $A$ is written a (potentially large) perturbation around a background configuration,

$$
A=A_{0}+\alpha
$$

From this one has the expansion of the field-strength of $A$,

$$
F=F_{0}+f+\frac{1}{2}[\alpha, \alpha],
$$

with $f:=D^{A_{0}} \alpha=D_{0} \alpha$. It is further supposed that the background satisfies the source-free YM equation $D_{0} * F_{0}=0$, with $F_{0}$ the field-strength of $A_{0}$, so that (B.1) is rewritten,

$$
\begin{aligned}
& D_{0 * F_{0}}+\underbrace{\left[\alpha, * F_{0}\right]+D_{0} * f}_{\text {linear in } \alpha}+\underbrace{[\alpha, * f]+D^{A} * \frac{1}{2}[\alpha, \alpha]}_{:=\{D * F\}^{R}, \text { order } \geq 2 \text { in } \alpha}=J, \\
& D_{0} * f+\left[\alpha, * F_{0}\right]=J-\{D * F\}^{R}:=j .
\end{aligned}
$$

The point is to prove the fact that, on-shell, the newly defined current satisfies a covariant conservation law w.r.t. the background. It is easily found that, on the one hand $D_{0} D_{0} * f=$ $\left[F_{0}, * f\right]$, and on the other hand

$$
D_{0}\left[\alpha, * F_{0}\right]=\left[D_{0} \alpha, * F_{0}\right]-\left[\alpha, D_{0} * F_{0}\right]=\left[f, * F_{0}\right] .
$$

Thus, applying $D_{0}$ on (B.4), one indeed gets the on-shell relation $D_{0} j \approx 0$.

Now the second ansatz is introduced, i.e. that one considers the Killing equation

$$
D_{0} \chi=0,
$$

so that $\chi$ is a symmetry of the background field $A_{0}$, and from which follows of course $D_{0} D_{0} \chi=\left[F_{0}, \chi\right]=0$. Then, from the $D_{0}$-conservation of $j$ and (B.6) one obtains

$$
\begin{aligned}
d \operatorname{Tr}(\chi j) & =\operatorname{Tr}(d \chi j)+\operatorname{Tr}(\chi d j)+\underbrace{\operatorname{Tr}\left(\left[A_{0}, \chi\right], j\right)+\operatorname{Tr}\left(\chi\left[A_{0}, j\right]\right)}_{=0 \text { by } \mathcal{H} \text {-invariance of } \operatorname{Tr}} \\
& =\operatorname{Tr}\left(D_{0} \chi j\right)+\operatorname{Tr}\left(\chi D_{0} j\right) \approx 0,
\end{aligned}
$$


We have then the conserved Noether current $J_{\chi}:=\operatorname{Tr}(\chi j)$, and integrated over the codimension 1 surface $\Sigma$, it gives the Noether charge $Q_{\chi}:=\int_{\Sigma} \operatorname{Tr}(\chi j)$ associated with the background Killing symmetry $\chi$.

But this is not over yet: using again the field equations (B.4), we get

$$
\begin{aligned}
Q_{\chi} & =\int_{\Sigma} \operatorname{Tr}(\chi j) \approx \int_{\Sigma} \operatorname{Tr}\left(\chi D_{0} * f\right)+\underbrace{\operatorname{Tr}\left(\chi\left[\alpha, * F_{0}\right]\right)}_{-\operatorname{Tr}\left([\alpha, \chi] * F_{0}\right)=0} \\
& =\int_{\Sigma} d \operatorname{Tr}(\chi * f)-\operatorname{Tr}\left(D_{\sigma} \chi * f\right)=\int_{\partial \Sigma} \operatorname{Tr}(\chi * f) .
\end{aligned}
$$

Which may be compared to eq. (3.38) in section 3.3.1. A final step can be taken to get,

$$
Q_{\chi}=\int_{\partial \Sigma} \operatorname{Tr}(\chi * f)=\int_{\partial \Sigma} * \operatorname{Tr}\left(\chi D_{0} \alpha\right)=\int_{\partial \Sigma} * d \operatorname{Tr}(\chi \alpha),
$$

using again the Killing equation. Abbott and Deser [34] remark that this, in component, generalises the electric charge in electrodynamics. It reduces to it indeed in the abelian case, as then the Killing equation is $d \chi=0$, so the constant gauge parameter exits the integral - and we recover the notion that the conservation of the electric charge results from a global (instead of gauge) abelian symmetry.

\section{Commutation relations with the extended bracket (3.20)}

The bracket under consideration is defined by $\{\boldsymbol{\chi}, \boldsymbol{\eta}\}:=[\boldsymbol{\chi}, \boldsymbol{\eta}]+\boldsymbol{\chi}^{v}(\boldsymbol{\eta})-\boldsymbol{\eta}^{v}(\boldsymbol{\chi})$ for $\phi$ dependent gauge parameters. In this paper we have $\boldsymbol{\chi}, \boldsymbol{\eta} \in$ Lie $\mathcal{H}$, which actually reduces the bracket to $-[\boldsymbol{\chi}, \boldsymbol{\eta}]$. But we do not make this simplification so as to keep the calculations valid for field-dependent diffeomorphisms. Let us compute, using (2.5),

$$
\left[\iota_{\chi^{v}}, \iota_{\boldsymbol{d}} \boldsymbol{\eta}^{v}\right] \boldsymbol{d} \phi=\iota_{\chi} \boldsymbol{\chi}_{\boldsymbol{d} \boldsymbol{\eta}} \phi-\iota_{\boldsymbol{d} \boldsymbol{\eta} \boldsymbol{\eta}^{v}} \delta_{\boldsymbol{\chi}} \phi=\delta_{\boldsymbol{d} \boldsymbol{\eta}\left(\chi^{v}\right)} \phi=\iota_{\left[\chi^{v}(\boldsymbol{\eta})\right]^{v}} \boldsymbol{d} \phi .
$$

As $\iota \boldsymbol{d} \boldsymbol{\eta}^{v}$ is manifestly a derivation of degree $0,\left[\iota_{\chi}, \iota_{\boldsymbol{d}} \boldsymbol{\eta}^{v}\right]$ is a derivation of degree -1 . The above result thus extends to arbitrary variational form: $\left[\iota_{\chi^{v}}, \iota_{\boldsymbol{d}} \boldsymbol{\eta}^{v}\right]=\iota_{\left[\boldsymbol{\chi}^{v}(\boldsymbol{\eta})\right]^{v}}$.

Now let us consider,

$$
\begin{aligned}
{\left[\boldsymbol{L}_{\boldsymbol{\chi}^{v}}, \iota_{\boldsymbol{\eta}^{v}}\right] \boldsymbol{d} \phi } & =\boldsymbol{L}_{\boldsymbol{\chi}^{v}} \delta_{\boldsymbol{\eta}} \phi-\iota_{\boldsymbol{\eta}^{v}} \boldsymbol{d}\left(\iota_{\chi^{v}} \boldsymbol{d} \phi\right)=\iota_{\chi^{v}} \boldsymbol{d}\left(\delta_{\boldsymbol{\eta}} \phi\right)-\iota_{\boldsymbol{\eta}^{v}} \boldsymbol{d}\left(\delta_{\boldsymbol{\chi}} \phi\right) \\
& =\iota_{\chi^{v}}\left(\delta_{\boldsymbol{d} \boldsymbol{\eta}} \phi+\delta_{\boldsymbol{\eta}} \boldsymbol{d} \phi\right)-\iota_{\boldsymbol{\eta}^{v}}\left(\delta_{\boldsymbol{d} \boldsymbol{\chi}} \phi+\delta_{\boldsymbol{\chi}} \boldsymbol{d} \phi\right) \\
& =\delta_{\boldsymbol{\chi}^{v}(\boldsymbol{\eta})} \phi+\delta_{\boldsymbol{\eta}} \delta_{\boldsymbol{\chi}} \phi-\delta_{\boldsymbol{\eta}^{v}(\boldsymbol{\chi})} \phi-\delta_{\boldsymbol{\chi}} \delta_{\boldsymbol{\eta}} \phi \\
& =\delta_{[\boldsymbol{\chi}, \boldsymbol{\eta}]} \phi+\delta_{\boldsymbol{\chi}^{v}(\boldsymbol{\eta})} \phi-\delta_{\boldsymbol{\eta}^{v}(\boldsymbol{\chi})} \phi \\
& =\iota_{\{\boldsymbol{\chi}, \boldsymbol{\eta}\}^{v}} \boldsymbol{d} \phi .
\end{aligned}
$$

The bracket of $\iota_{\chi}$ and $\iota_{\boldsymbol{d}} \eta^{v}$ is a derivation of degree -1 that extends to any form, so $\left[\boldsymbol{L}_{\boldsymbol{\chi}^{v}}, \iota_{\boldsymbol{\eta}^{v}}\right]=\iota_{\{\boldsymbol{\chi}, \boldsymbol{\eta}\}^{v}}$. From this and $\left[\boldsymbol{L}_{\boldsymbol{\chi}^{v}}, \boldsymbol{d}\right]=0$ follows that,

$$
\begin{aligned}
{\left[\boldsymbol{L}_{\boldsymbol{\chi}^{v}}, \boldsymbol{L}_{\boldsymbol{\eta}^{v}}\right] } & =\left[\boldsymbol{L}_{\boldsymbol{\chi}^{v}}, \iota_{\boldsymbol{\eta}^{v}} \boldsymbol{d}+\boldsymbol{d} \iota_{\boldsymbol{\eta}^{v}}\right], \\
& =\boldsymbol{L}_{\boldsymbol{\chi}^{v} \iota_{\boldsymbol{\eta}^{v}}} \boldsymbol{d}-\iota_{\boldsymbol{\eta}^{v}} \boldsymbol{d} \boldsymbol{L}_{\boldsymbol{\chi}^{v}}+\boldsymbol{L}_{\boldsymbol{\chi}^{v}} \boldsymbol{d} \iota_{\boldsymbol{\eta}^{v}}-\boldsymbol{d} \iota_{\boldsymbol{\eta}^{v}} \boldsymbol{L}_{\boldsymbol{\chi}^{v}} \\
& =\boldsymbol{L}_{\boldsymbol{\chi}^{v} \iota_{\boldsymbol{\eta}^{v}}} \boldsymbol{d}-\iota_{\boldsymbol{\eta}^{v}} \boldsymbol{L}_{\boldsymbol{\chi}^{v}} \boldsymbol{d}+\boldsymbol{d} \boldsymbol{L}_{\boldsymbol{\chi}^{v} \iota_{\boldsymbol{\eta}^{v}}}-\boldsymbol{d} \iota_{\boldsymbol{\eta}^{v}} \boldsymbol{L}_{\boldsymbol{\chi}^{v}} \\
& =\left[\boldsymbol{L}_{\boldsymbol{\chi}^{v}}, \iota_{\boldsymbol{\eta}^{v}}\right] \boldsymbol{d}+\boldsymbol{d}\left[\boldsymbol{L}_{\boldsymbol{\chi}^{v} \iota} \iota_{\boldsymbol{\eta}^{v}}\right]=\iota_{\{\boldsymbol{\chi}, \boldsymbol{\eta}\}^{v}} \boldsymbol{d}+\boldsymbol{d} \iota_{\{\boldsymbol{\chi}, \boldsymbol{\eta}\}^{v}} \\
& =\boldsymbol{L}_{\{\boldsymbol{\chi}, \boldsymbol{\eta}\}^{v}} .
\end{aligned}
$$


Open Access. This article is distributed under the terms of the Creative Commons Attribution License (CC-BY 4.0), which permits any use, distribution and reproduction in any medium, provided the original author(s) and source are credited.

\section{References}

[1] G.J. Zuckerman, Action principles and global geometry, Conf. Proc. C 8607214 (1986) 259 [INSPIRE].

[2] C. Crnkovic and E. Witten, Three hundred years of gravitation, Cambridge University Press, Camrbidge U.K. (1987).

[3] C. Crnkovic, Symplectic geometry of the covariant phase space, superstrings and superspace, Class. Quant. Grav. 5 (1988) 1557 [INSPIRE].

[4] F. Gieres, Covariant canonical formulations of classical field theories, arXiv:2109.07330 [INSPIRE].

[5] W. Donnelly and L. Freidel, Local subsystems in gauge theory and gravity, JHEP 09 (2016) 102 [arXiv: 1601.04744$]$ [INSPIRE].

[6] H. Gomes and A. Riello, The observer's ghost: notes on a field space connection, JHEP 05 (2017) 017 [arXiv: 1608.08226] [INSPIRE].

[7] H. Gomes and A. Riello, Unified geometric framework for boundary charges and particle dressings, Phys. Rev. D 98 (2018) 025013 [arXiv:1804.01919] [InSPIRE].

[8] H. Gomes, F. Hopfmüller and A. Riello, A unified geometric framework for boundary charges and dressings: non-Abelian theory and matter, Nucl. Phys. B 941 (2019) 249 [arXiv: 1808.02074] [INSPIRE].

[9] H. Gomes and A. Riello, The quasilocal degrees of freedom of Yang-Mills theory, SciPost Phys. 10 (2021) 130 [arXiv: 1910.04222] [INSPIRE].

[10] H. Gomes, Gauging the boundary in field-space, Studies History Phil. Sci. B 67 (2019) 89.

[11] H. Gomes, Holism as the empirical significance of symmetries, Eur. J. Phil. Sci. 11 (2021) 87 [arXiv: 1910.05330] [INSPIRE].

[12] A. Riello, Soft charges from the geometry of field space, JHEP 05 (2020) 125 [arXiv: 1904.07410] [INSPIRE].

[13] A. Riello, Symplectic reduction of Yang-Mills theory with boundaries: from superselection sectors to edge modes, and back, SciPost Phys. 10 (2021) 125 [arXiv:2010.15894] [INSPIRE].

[14] M. Geiller, Edge modes and corner ambiguities in 3d Chern-Simons theory and gravity, Nucl. Phys. B 924 (2017) 312 [arXiv:1703. 04748] [InSPIRE].

[15] M. Geiller, Lorentz-diffeomorphism edge modes in 3d gravity, JHEP 02 (2018) 029 [arXiv: 1712.05269] [INSPIRE].

[16] A.J. Speranza, Local phase space and edge modes for diffeomorphism-invariant theories, JHEP 02 (2018) 021 [arXiv:1706. 05061] [INSPIRE].

[17] M. Geiller and P. Jai-akson, Extended actions, dynamics of edge modes, and entanglement entropy, JHEP 09 (2020) 134 [arXiv: 1912.06025] [InSPIRE]. 
[18] A. Balachandran, L. Chandar and E. Ercolessi, Edge states in gauge theories: Theory, interpretation and predictions, Int. J. Mod. Phys A 10 (1995) 1969.

[19] A.P. Balachandran, L. Chandar and A. Momen, Edge states in gravity and black hole physics, Nucl. Phys. B 461 (1996) 581 [gr-qc/9412019] [InSPIRE].

[20] M. Asorey, A.P. Balachandran and J.M. Pérez-Pardo, Edge states at phase boundaries and their stability, Rev. Math. Phys. 28 (2016) 1650020.

[21] H. Adami, M.M. Sheikh-Jabbari, V. Taghiloo, H. Yavartanoo and C. Zwikel, Symmetries at null boundaries: two and three dimensional gravity cases, JHEP 10 (2020) 107 [arXiv:2007.12759] [INSPIRE].

[22] A. Seraj, Gravitational breathing memory and dual symmetries, JHEP 05 (2021) 283 [arXiv:2103.12185] [INSPIRE].

[23] L. Freidel, M. Geiller and D. Pranzetti, Edge modes of gravity. Part I. Corner potentials and charges, JHEP 11 (2020) 026 [arXiv:2006.12527] [INSPIRE].

[24] L. Freidel, M. Geiller and D. Pranzetti, Edge modes of gravity. Part II. Corner metric and Lorentz charges, JHEP 11 (2020) 027 [arXiv:2007.03563] [INSPIRE].

[25] L. Freidel, M. Geiller and D. Pranzetti, Edge modes of gravity. Part III. Corner simplicity constraints, JHEP 01 (2021) 100 [arXiv:2007.12635] [INSPIRE].

[26] M. Lavelle and D. McMullan, Constituent quarks from QCD, Phys. Rept. 279 (1997) 1.

[27] E. Bagan, M. Lavelle and D. McMullan, Charges from dressed matter: construction, Ann. Phys. 282 (2000) 471.

[28] E. De Paoli and S. Speziale, A gauge-invariant symplectic potential for tetrad general relativity, JHEP 07 (2018) 040 [arXiv: 1804.09685] [INSPIRE].

[29] R. Oliveri and S. Speziale, Boundary effects in general relativity with tetrad variables, Gen. Rel. Grav. 52 (2020) 83 [arXiv:1912.01016] [INSPIRE].

[30] R. Oliveri and S. Speziale, A note on dual gravitational charges, JHEP 12 (2020) 079 [arXiv:2010.01111] [INSPIRE].

[31] S. Murgueitio Ramírez and N. Teh, Abandoning Galileo's ship: the quest for non-relational empirical significance, British J. Phys. Sci. (2020).

[32] P. Mathieu, L. Murray, A. Schenkel and N.J. Teh, Homological perspective on edge modes in linear Yang-Mills and Chern-Simons theory, Lett. Math. Phys. 110 (2020) 1559.

[33] J. François, Bundle geometry of the connection space, covariant Hamiltonian formalism, the problem of boundaries in gauge theories, and the dressing field method, JHEP 03 (2021) 225 [arXiv:2010.01597] [INSPIRE].

[34] L.F. Abbott and S. Deser, Charge definition in nona-Belian gauge theories, Phys. Lett. B 116 (1982) 259 [INSPIRE].

[35] L.F. Abbott and S. Deser, Stability of gravity with a cosmological constant, Nucl. Phys. B 195 (1982) 76 [INSPIRE].

[36] G. Barnich and F. Brandt, Covariant theory of asymptotic symmetries, conservation laws and central charges, Nucl. Phys. B 633 (2002) 3 [hep-th/0111246] [INSPIRE].

[37] A. Frölicher and A. Kriegl, Linear spaces and differentiation theory, Pure and Applied Mathematics, John Wiley \& Sons, U.S.A. (1988). 
[38] A. Kriegl and P.P. Michor, The convenient setting of global analysis, Mathematical Surveys and Monographs volume 53, American Mathematical Society, U.S.A. (1997).

[39] I.M. Singer, Some remarks on the Gribov ambiguity, Commun. Math. Phys. 60 (1978) 7 [INSPIRE].

[40] I.M. Singer, The geometry of the orbit space for non-abelian gauge theories, Phys. Scripta 24 (1981) 817.

[41] P.K. Mitter and C.M. Viallet, On the bundle of connections and the gauge orbit manifold in yang-mills theory, Commun. Math. Phys. 79 (1981) 457.

[42] P. Cotta Ramusino and C. Reina, The action of the group of bundle-automorphisms on the space of connections and the geometry of gauge theories, J. Geom. Phys. 1 (1984) 121.

[43] M. Abbati, R. Cirelli, A. Maniá and P. Michor, The Lie group of automorphisms of a principle bundle, J. Geom. Phys. 6 (1989) 215.

[44] J. Fuchs, The singularity structure of the Yang-Mills configuration space, Banach Center Publ. 39 (1997) 287.

[45] V.N. Gribov, Quantization of nonabelian gauge theories, Nucl. Phys. B 139 (1978) 1 [INSPIRE].

[46] B.S. DeWitt, The global approach to quantum field theory, International series of monographs on physics, Oxford University Press, Oxford U.K. (2003).

[47] J. François, Twisted gauge fields, arXiv:1907.08666 [INSPIRE].

[48] R.A. Bertlmann, Anomalies in quantum field theory, International Series of Monographs on Physics volume 91, Oxford University Press, Oxford U.K. (1996).

[49] M. Göckeler and T. Schücker, Differential geometry, gauge theory and gravity, Cambridge Monographs on Mathematical Physics, Cambridge University Press, Cambridge U.K. (1987).

[50] J. Mañes, R. Stora and B. Zumino, Algebraic study of chiral anomalies, Commun. Math. Phys. 102 (1985) 157 [INSPIRE].

[51] J. Attard and S. Lazzarini, A note on Weyl invariance in gravity and the Wess-Zumino functional, Nucl. Phys. B 912 (2016) 289 [arXiv:1607.06326] [INSPIRE].

[52] C. Fournel, J. François, S. Lazzarini and T. Masson, Gauge invariant composite fields out of connections, with examples, Int. J. Geom. Meth. Phys. 11 (2014) 1450016.

[53] J. François, Reduction of gauge symmetries: a new geometrical approach, thesis, Aix-Marseille Université, Fracne (2014).

[54] J. Attard, J. François, S. Lazzarini and T. Masson, The dressing field method of gauge symmetry reduction, a review with examples, arXiv:1702.02753 [INSPIRE].

[55] J. François, Artificial versus substantial gauge symmetries: a criterion and an application to the electroweak model, Phil. Sci. 86 (2019) 472.

[56] F. Hélein, Variational problems in differential geometry, London Mathematical Society Lecture Note Series volume 394, Cambridge University Press, Cambridge U.K. (2012).

[57] J. Lee and R. Wald, Local symmetries and constraints, J. Math. Phys. 31 (1990) 725. 
[58] A. Ashtekar, L. Bombelli and O. Reula, The covariant phase space of asymptotically flat gravitational fields, in Mechanics, analysis and geometry: 200 years after lagrange, M. Francaviglia ed., North-Holland Delta Series, Elsevier, Switzerland (1991).

[59] G. Compère and A. Fiorucci, Advanced lectures on general relativity, arXiv:1801.07064 [INSPIRE].

[60] D. Harlow and J.-Q. Wu, Covariant phase space with boundaries, JHEP 10 (2020) 146 [arXiv: 1906.08616] [INSPIRE].

[61] H. Farajollahi and H. Lückock, Dirac observables and the phase space of general relativity, Gen. Rel. Grav. 34 (2002) 1685 [gr-qc/0112051] [inSPIRE].

[62] M. Castrillón López, J. Muñoz Masqué and E. Rosado María, Structure of gauge-invariant Lagrangians, Mediterr. J. Math. 17 (2019) 29.

[63] U. Bruzzo, The global Utiyama theorem in Einstein-Cartan theory, J, Math. Phys. 28 (1987) 2074.

[64] M. Forger and S. Viera Romero, Covariant Poisson brackets in geometric field theory, Commun. math. Phys. 256 (2005) 375.

[65] L. Freidel, R. Oliveri, D. Pranzetti and S. Speziale, Extended corner symmetry, charge bracket and Einstein's equations, JHEP 09 (2021) 083 [arXiv:2104.12881] [INSPIRE].

[66] G. Barnich, F. Brandt and M. Henneaux, Local BRST cohomology in gauge theories, Phys. Rept. 338 (2000) 439 [hep-th/0002245] [INSPIRE].

[67] R.W. Sharpe, Differential geometry: Cartan's generalization of Klein's Erlangen program, Graduate text in Mathematics volume 166, Springer, Germany (1996).

[68] A. Cap and J. Slovak, Parabolic geometries i: background and general theory, Mathematical Surveys and Monographs volume 1, American Mathematical Society, U.S.A. (2009).

[69] S. Carrozza and P.A. Hoehn, Edge modes as reference frames and boundary actions from post-selection, arXiv:2109.06184 [INSPIRE].

[70] P.A.M. Dirac, Gauge-invariant formulation of quantum electrodynamics, Canad. J. Phys. 33 (1955) 650.

[71] P.A.M. Dirac, The principles of quantum mechanics, ${ }^{\text {th }}$ edition, Oxford University Press, Oxford, U.K. (1958).

[72] J.B. Pitts, Empirical equivalence, artificial gauge freedom and a generalized Kretschmann objection, arXiv:0911.5400 [INSPIRE].

[73] R. Jackiw and S.-Y. Pi, Fake conformal symmetry in conformal cosmological models, Phys. Rev. D 91 (2015) 067501 [arXiv:1407.8545] [INSPIRE].

[74] P. Berghofer et al., Gauge symmetries, symmetry breaking, and gauge-invariant approaches, arXiv:2110.00616 [INSPIRE].

[75] H. Lyre, Holism and structuralism in U(1) gauge theory, Studies History Phil. Mod. Phys. 35 (2004) 643.

[76] A. Guay, A partial elucidation of the gauge principle, Studies History Phil. Sci. B 39 (2008) 346.

[77] R. Healey, Gauging what's real: the conceptual foundation of contemporary gauge theories, Oxford University Press, Oxford U.K. (2009). 
[78] J. Dougherty, Sameness and separability in gauge theories, Phil. Sci. 84 (2017) 1189.

[79] J. Nguyen, N.J. Teh and L. Wells, Why surplus structure is not superfluous, British J. Phys. Sci. 71 (2020) 665.

[80] A. Riello, Edge modes without edge modes, arXiv:2104.10182 [INSPIRE].

[81] D. Wallace, Deflating the Aharonov-Bohm effect, arXiv:1407.5073 [INSPIRE].

[82] T. Masson and J.-C. Wallet, A remark on the spontaneous symmetry breaking mechanism in the standard model, arXiv:1001.1176 [INSPIRE].

[83] P.W. Higgs, Spontaneous symmetry breakdown without massless bosons, Phys. Rev. 145 (1966) 1156 [INSPIRE].

[84] T.W.B. Kibble, Symmetry breaking in non-Abelian gauge theories, Phys. Rev. 155 (1967) 1554 [INSPIRE].

[85] T. Banks and E. Rabinovici, Finite temperature behavior of the lattice abelian Higgs model, Nucl. Phys. B 160 (1979) 349 [INSPIRE].

[86] J. Fröhlich, G. Morchio and F. Strocchi, Higgs phenomenon without symmetry breaking order parameter, Nucl. Phys. B 190 (1981) 553 [InSPIRE].

[87] W. Buchmüller, Z. Fodor and A. Hebecker, Gauge invariant treatment of the electroweak phase transition, Phys. Lett. B 331 (1994) 131 [hep-ph/9403391] [INSPIRE].

[88] M. Lavelle and D. McMullan, Observables and gauge fixing in spontaneously broken gauge theories, Phys. Lett. B 347 (1995) 89 [hep-th/9412145] [INSPIRE].

[89] L.D. Faddeev, An alternative interpretation of the Weinberg-Salam model, in Progress in High Energy Physics and Nuclear Safety, V. Begun et al. eds., Springer, Germany (2009).

[90] A. Ilderton, M. Lavelle and D. McMullan, Symmetry breaking, conformal geometry and gauge invariance, J. Phys. A 43 (2010) 312002 [arXiv:1002.1170] [InSPIRE].

[91] A. Maas, Brout-Englert-Higgs physics: from foundations to phenomenology, Progr. Part. Nucl. Phys. 106 (2019) 132.

[92] J. Earman, Curie's principle and spontaneous symmetry breaking, Int. Studies Phil. Sci. 18 (2004) 173.

[93] C. Smeenk, The elusive Higgs mechanism, Phil. Sci. 73 (2006) 487.

[94] H. Lyre, Does the Higgs mechanism exists?, Int. Studies Phil. Sci. 22 (2008) 119.

[95] W. Struyve, Gauge invariant accounts of the higgs mechanism, Studies History Phil. Sci. B 42 (2011) 226.

[96] S. Friederich, Gauge symmetry breaking in gauge theories - In search of clarification, Eur. J. Phil. Sci. 3 (2013) 157.

[97] S. Friederich, A philosophical look at the Higgs mechanism, J. Gen. Philos. Sci 45 (2014) 335.

[98] D. Kastor, Komar integrals in higher (and lower) derivative gravity, Class. Quant. Grav. 25 (2008) 175007 [arXiv:0804.1832] [INSPIRE].

[99] Y. Choquet-Bruhat, General relativity and the Einstein equations, Oxford Mathematical Monographs, Oxford University Press, Oxford U.K. (2009). 
[100] J.B. Pitts, The nontriviality of trivial general covariance: How electrons restrict time coordinates, spinors (almost) fit into tensor calculus, and 7/16 of a tetrad is surplus structure, Studies History Phil. Mod. Phys. 43 (2012) 1 [arXiv:1111.4586].

[101] S.N. Curry and A.R. Gover, An introduction to conformal geometry and tractor calculus, with a view to applications in general relativity, in Asymptotic analysis in general relativity, T. Daudé et al. eds., London Mathematical Society, Cambridge University Press, Cambridge U.K. (2018).

[102] G. Barnich and C. Troessaert, BMS charge algebra, JHEP 12 (2011) 105 [arXiv: 1106.0213] [INSPIRE].

[103] G. Compère, A. Fiorucci and R. Ruzziconi, The $\Lambda$-BMS $S_{4}$ charge algebra, JHEP 10 (2020) 205 [arXiv: 2004.10769] [INSPIRE].

[104] L. Freidel, R. Oliveri, D. Pranzetti and S. Speziale, The Weyl BMS group and Einstein's equations, JHEP 07 (2021) 170 [arXiv:2104.05793] [INSPIRE]. 